\title{
Supramolecular Stimuli-Responsive Microgels Crosslinked by Tannic Acid
}

\section{Supramolekulare stimuli-responsive Mikrogele vernetzt mit Tanninsäure}

Von der Fakultät für Mathematik, Informatik und Naturwissenschaften der RWTH Aachen University zur Erlangung des akademischen Grades einer Doktorin der

Naturwissenschaften genehmigte Dissertation

vorgelegt von

M. Sc.

\section{Astrid Catalina Molano López}

aus Bogota-Kolumbien

Berichter: Universitätsprofessor Dr. rer. nat. Andrij Pich

Universitätsprofessor Dr. rer. nat. Walter Richtering

Tag der mündlichen Prüfung: 15.02.2019 
The scientific work presented in this doctoral thesis was performed at the DWI-Leibniz Institute for Interactive Materials e.V. and the RWTH Aachen University from April 2015 until November 2018 under the supervision of Prof. Dr.rer.nat. Andrij Pich. 


\section{Eidesstattliche Erklärung}

Hiermit erkläre ich, Astrid Catalina Molano López, dass ich diese Dissertation eigenständig verfasst und nur die angegebenen Quellen und Hilfsmitteln verwendet habe. Diese Promotionsarbeit wurde bisher nicht veröffentlicht und noch keiner Prüfungsbehörde vorgelegt. Teile dieser Dissertation wurden in einer wissenschaftlichen Zeitschrift so wie in Konferenzbeiträgen publiziert. (siehe: „LIST OF PUBLICATIONS AND CONFERENCE CONTRIBUTIONS")

Ort, Datum, Unterschrift 
"I learned to not be intimidated by the problem"

Prof. Paula T. Hammond 


\section{Contents}

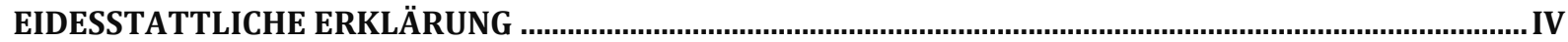

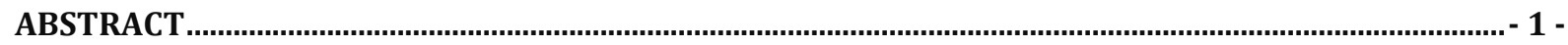

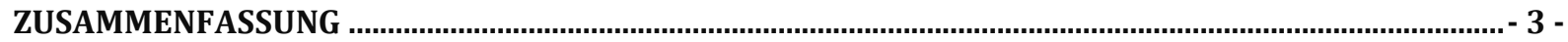

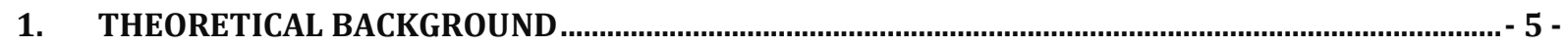

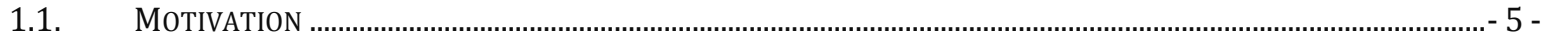

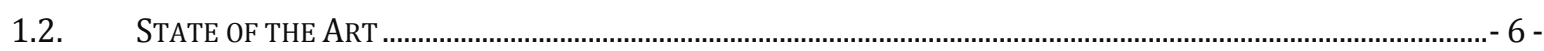

1.2.1. Polymer-based Biomaterials.....................................................................................................

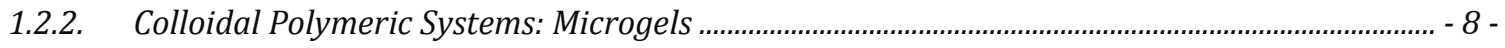

1.2.3. Supramolecular Nanomaterials ........................................................................................................ - 10 -

1.2.4. Bioactive Polyphenols........................................................................................................ 12 -

1.2.5. Tannic Acid: Structure and Properties.............................................................................................- 14 -

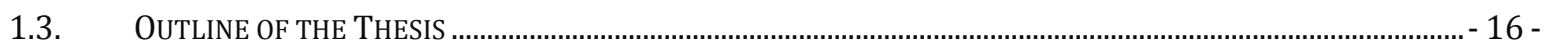

2. TANNIC ACID AS SUPRAMOLECULAR CROSSLINKER FOR PVCL-MICROGELS SYNTHESIZED BY BATCH PRECIPITATION POLYMERIZATION.............................................................................................. - 18 -

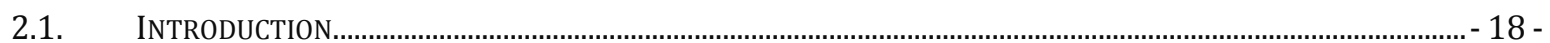

2.2. RESULTS AND DISCUSSION ……………………………......................................................................... 19 -

2.2.1. PROOF OF CONCEPT: TA INTERACTIONS.................................................................................................. 19 -

2.2.2. PRELIMINARY SYNTHESIS OF PVCL/TA-MICROGELS................................................................................... - 24 -

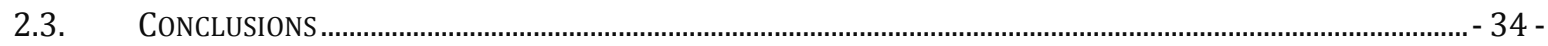

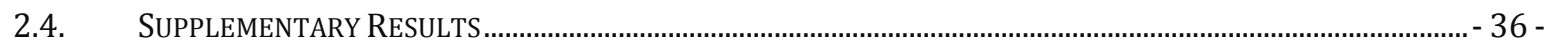

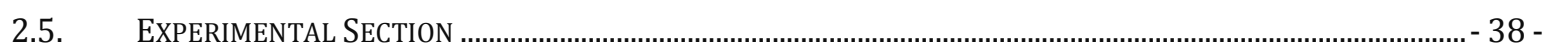

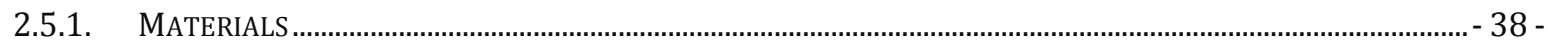

2.5.2. ANALYSIS OF TA INTERACTIONS AS PROOF OF CONCEPT ................................................................................. - 38 -

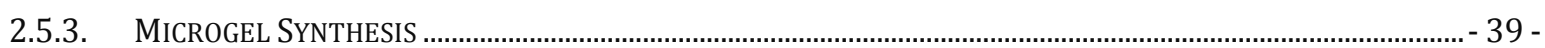

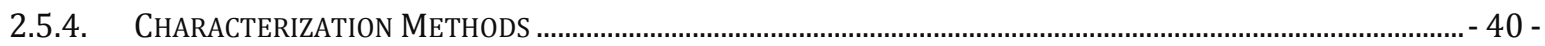

3. SYNTHESIS OF SUPRAMOLECULAR PVCL/TA-MICROGELS BY AN OPTIMIZED SEMI-BATCH PRECIPITATION POLYMERIZATION APPROACH ....................................................................... 43 -

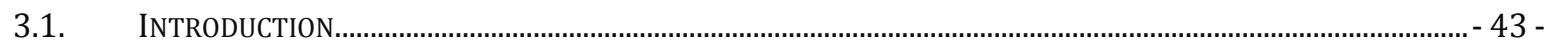

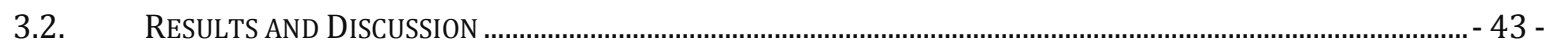

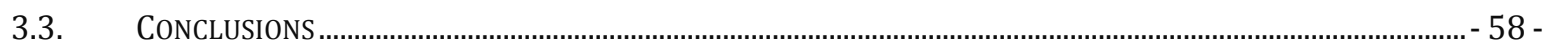

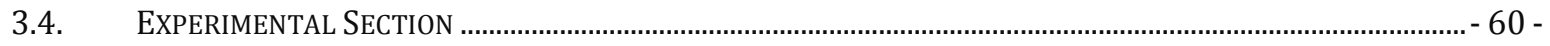

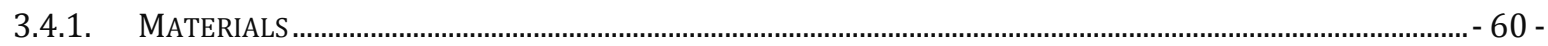

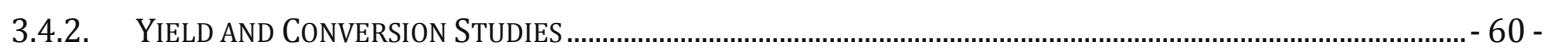

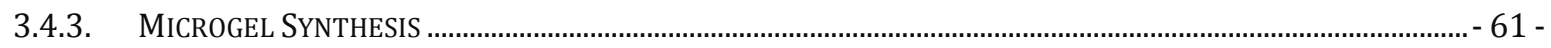




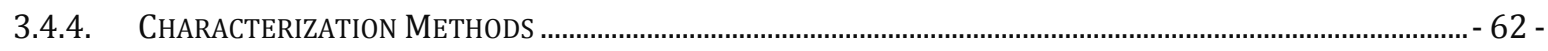

3.4.5. STUDY OF MICROGEL STABILITY IN ORGANIC SOLVENTS ............................................................................. 65 -

4. PH-TRIGGERED DISASSEMBLY OF SUPRAMOLECULAR MICROGELS ....................................... - 66 -

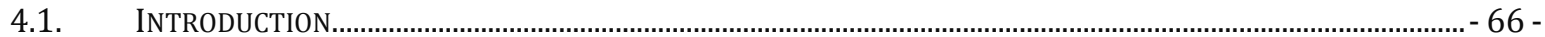

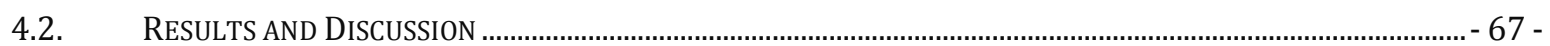

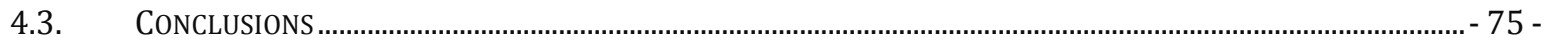

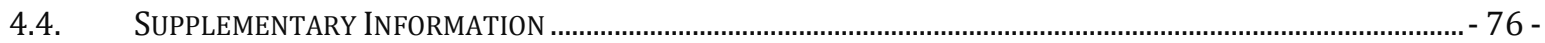

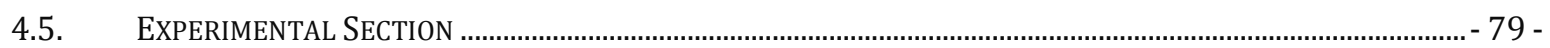

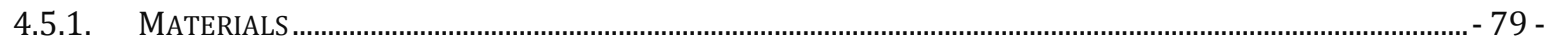

4.5.2. PH-DiSASSEMBLY OF THE PVCL/TA-MiCROGELS ............................................................................... 79

5. SUPRAMOLECULAR MICROGELS AS CARRIERS FOR ACTIVE COMPOUNDS ............................. - 81 -

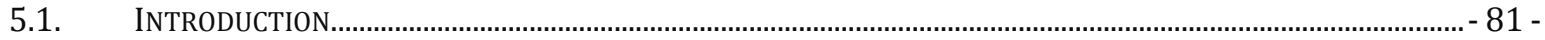

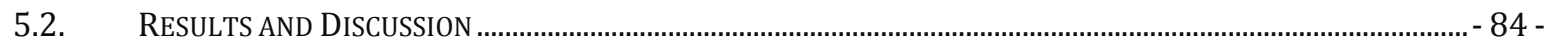

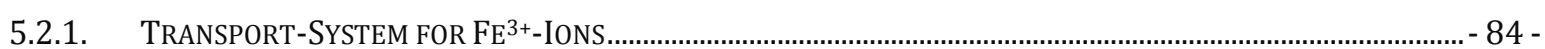

5.2.2. InCORPORATION OF OTHER (POLY-) PHENOLIC COMPOUNDS...................................................................... 88 -

5.2.3. ENCAPSULATION OF ANTIBACTERIAL ALKYL GALLATES ............................................................................. 95 -

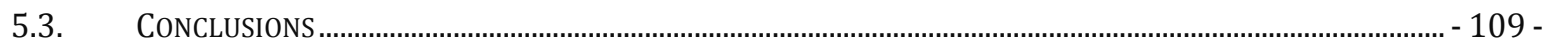

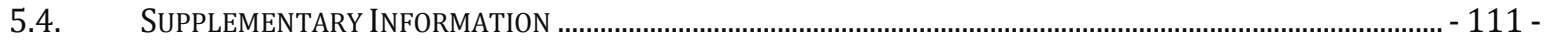

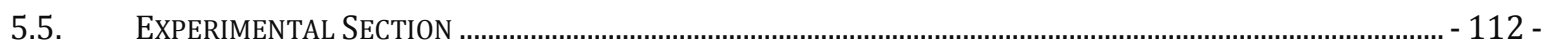

5.5.1. MATERIALS ............................................................................................................................................. 112

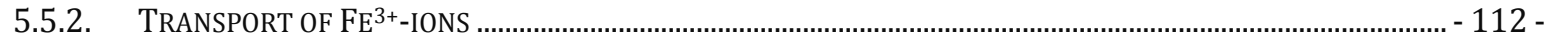

5.5.3. INCORPORATION OF FURTHER PHENOLIC COMPOUNDS AND ANTIBACTERIAL ALKYL GALLATES................. - 113 -

5.5.4. CHARACTERIZATION METHODS ……............................................................................................... 114

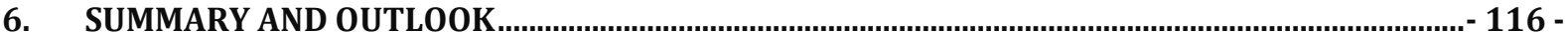

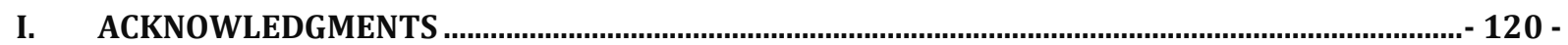

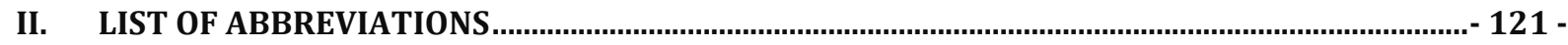

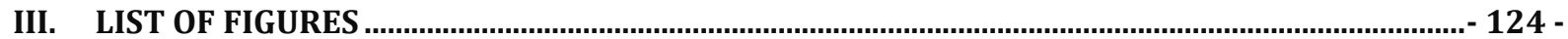

IV. LIST OF TABLES ................................................................................................................ 127

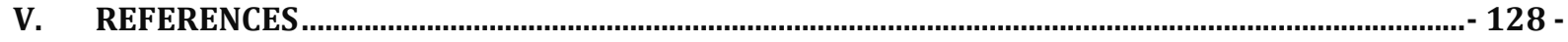

VI. LIST OF PUBLICATIONS AND CONFERENCE CONTRIBUTIONS ................................................ 140 - 



\begin{abstract}
After the era of revolutionary industrial developments, our society has reached the "green" period, in which the production of bioinspired materials is a prior ambition. Therefore, the understanding of biological processes at the molecular level opens up great possibilities and guides the evolutional path in modern science. Whether at the nanometric or at the macroscopic scale, the origin of living entities relies almost always on self-assembly and hierarchic organization between building blocks. These mechanisms and the role that biological units play herein are the inspiration in design of novel "smart" nanomaterials for bio- and medical applications. Additionally, the utilization of natural compounds in material science is also desired.
\end{abstract}

Among the great variety of promising materials, polymers have showed remarkable biocompatible and responsive properties. In particular, polymeric colloidal networks, well-known as microgels, have been arousing enormous interest because of their stimuli-responsiveness and biocompatibility depending on their building units. Herein, not only the selected monomer, but also the crosslinking agent plays a decisive role, especially in view of supramolecular design. Supramolecular polymeric materials that allow adaptation, molecular recognition and triggered disassociation have been developed successfully within the last years. However, in terms of supramolecular microgels there are only few reports published, and all of them imply multistep procedures, as well as complex syntheses of supramolecular precursors. Based on all these facts, the main goal of this thesis was the design of bio-based supramolecular microgels with reversible crosslinks, and their utilization in bio-applications.

In this work, I present a facile and optimized approach to produce supramolecular stimuli-responsive microgels composed of thermoresponsive and biocompatible poly $(N$ vinylcaprolactam) (PVCL) and the bioactive natural polyphenol tannic acid (TA). The ability of TA to form hydrogen bonds with polymeric compounds, allows its facile incorporation as a physical crosslinker within the microgel synthesis. Depending on the TA content, the microgel properties such as chemical composition, deformability, crosslinking density and dimension can be controlled. Moreover besides thermoresponsiveness, the PVCL/TA-microgels exhibit pH-sensitivity and can be degraded easily by pH-triggered disintegration of the non-covalent bonds. This feature, 
leads simultaneously to a controlled TA-release. Furthermore, the pH-dependent behavior of the microgels in combination with the complexant capacity of the incorporated TA guides to different interesting applications. The supramolecular polyphenolic PVCL-microgels were thus studied as attractive $\mathrm{pH}$-responsive carriers for the transport of active components. Firstly, the nanomaterials were proposed as potential vehicles for the transport of metal ions $\left(\mathrm{Fe}^{3+}\right)$. Herein, a controlled loading and release of the metal ions was successfully demonstrated. Moreover, the PVCL/TAmicrogels were used as supramolecular containers for the incorporation of further phenolic derivatives, and encapsulation of antibacterial bioactive compounds. Here, the antibacterial activity of the multifunctional system was preliminary studied. The results obtained in this thesis confirmed not only that the developed microgel system is based on supramolecular assembly, but also that it offers an innovative, biocompatible and responsive compartment for the encapsulation of hydrophobic components in a waterbased nanomaterial. This unique microgel represents an innovative biocompatible system that can be adapted to different applications and needs. 


\section{Zusammenfassung}

Nach der Ära der revolutionären industriellen Entwicklungen hat unsere Gesellschaft die "grüne" Epoche erreicht, in der die Produktion von bioinspirierten Materialien ein primäres Ziel ist. Daher ermöglicht das Verständnis biologischer Prozesse auf molekularer Ebene großartige Möglichkeiten in der Forschung und leitet den Evolutionspfad der modernen Wissenschaft an. Ob in Nanometermaßstab oder im makroskopischen Größenordungen, der Ursprung des Lebens basiert fast immer auf Selbstorganisation und hierarchischer Anordnung von Bausteinen. Diese Mechanismen und die Rolle, welche biologische Einheiten hierbei spielen, sind die Inspiration bei der Entwicklung neuartiger "smarter" Nanomaterialien für bio- und medizinische Anwendungen. Zusätzlich ist ebenfalls die Verwendung von Naturstoffen in der Materialwissenschaft erwünscht.

Unter der großen Vielfalt vielversprechender Materialien haben Polymere bedeutende Eigenschaften in dieser Hinsicht gezeigt. Insbesondere haben Mikrogele, polymerbasierte kolloidale Netzwerke, aufgrund ihrer Empfindlichkeit gegenüber verschiederner Stimuli und Biokompatibilität, die von ihren Baueinheiten abhängen, ein enormes Interesse hervorgerufen. Hier spielt nicht nur das ausgewählte Monomer, sondern auch das Vernetzungsmittel eine entscheidende Rolle, insbesondere im Hinblick auf supramolekularem Design. Supramolekulare Polymer-basierte Materialien wurden in den letzten Jahren erfolgreich entwickelt und somit wurden bio-ähnliche Eigenschaften wie Adaptation, molekulare Erkennung und getriggerte Dissoziation ermöglicht. Im Hinblick auf supramolekulare Mikrogele gibt es jedoch nur wenige Veröffentlichungen, die alle mehrstufige Verfahren sowie komplexe Synthesen von supramolekularen Vorstufen implizieren. Aus diesen Gründen war das Hauptziel dieser Arbeit die Entwicklung von biobasierten supramolekularen Mikrogelen mit reversibler Vernetzung und schließlich deren Einsatz in Bio-Anwendungen.

In dieser Arbeit wird eine einfache und optimierte Methode zur Herstellung supramolekularer sensitiver Mikrogele präsentiert, die aus thermoresponsivem und biokompatiblem Poly( $N$-vinylcaprolactam) (PVCL) und der bioaktiven natürlichen Polyphenol Tanninsäure (TA) bestehen. 
Die Fähigkeit von TA, Wasserstoffbrückenbindungen mit Polymerverbindungen zu bilden, ermöglicht den leichten Einbau dieses Makromoleküls als physikalischer Vernetzer innerhalb der Mikrogel-Synthese. Abhängig vom TA-Gehalt können die Mikrogel-Eigenschaften, wie chemische Zusammensetzung, Vernetzungsdichte und Größe gesteuert werden. Darüber hinaus weisen die PVCL/TA-Mikrogele neben Thermoresponsivität ein pH-sensitives Verhalten auf und können durch pH-gesteuerte Desintegration der nicht-kovalenten Bindungen leicht abgebaut werden. Diese Eigenschaft führt gleichzeitig zur kontrollierten TA-Freisetzung. Des Weiteren weist das pH-abhängige Verhalten der Mikrogele in Kombination mit der Komplexierungskapazität der eingebauten TA auf verschiedene interessante Anwendungen hin. Die supramolekularen Polyphenol-basierten Mikrogele wurden daher als attraktive pH-empfindliche Träger für den Transport bioaktiver Komponenten untersucht. An erster Stelle wurden die Nanomaterialien für den Transport von Metallionen $\left(\mathrm{Fe}^{3+}\right)$ vorgeschlagen. Weiterhin wurden die PVCL/TA-Mikrogele als supramolekulare Container für den Einbau weiterer Phenol-Derivate und die Einkapselung antibakterieller bioaktiver Verbindungen eingesetzt. Hierbei wurde die antibakterielle Aktivität des multifunktionellen Systems vorläufig untersucht.

Die in dieser Arbeit erzielten Ergebnisse bestätigten nicht nur, dass das entwickelte Mikrogel-System auf supramolekularer Anordnung beruht, sondern auch, dass es ein innovatives, biokompatibles Kompartiment für die Einkapselung von hydrophoben Komponenten in einem wasserbasierten Nanomaterial bietet. Das einzigartige Mikrogel stellt somit ein neuartiges biokompatibles System dar, welches an unterschiedliche Anwendungen und Bedürfnisse angepasst werden kann. 


\section{Theoretical Background}

\subsection{Motivation}

Human evolution is described trough different anatomical adaptations, including the ability to reasoning and thinking. Additionally to the basic body functions, our species has developed the cognitive faculty strongly. The mental process is the origin of our curiosity and interest to understanding the world around us. Since the beginning of our history, innumerable revolutionary technologies have been developed by observing and understanding different phenomena. Control of fire, production of electricity, synthesis of antibiotics and vaccines, as well as the internet are some of the most significant discoveries. These did not only have a substantial impact on our life conditions, but also encouraged us to explore further innovations that improve our life quality even more. ${ }^{1-3}$ The design of "green materials" with properties comparable to natural systems and bioorganisms is hence, one of the most great scientific ambitions today. Since natural components such as cells and bacteria, as well as their smaller building blocks like proteins and peptides are found under the micrometer scale, bottom-up and atom-byatom synthetic approaches have been designed, and play an essential role in nanotechnology. 1, 4-8 This involves the evolvement of new systems at the nanometer range, a dimension in which matter changes drastically and unique properties can be achieved.6-7 In view of biomedical applications, the nanotechnology implies the combination of biology, polymer science and engineering among other research fields. There are a large number of key aspects which are essential to understand natural processes at the molecular level. Self-assembly, molecular recognition and supramolecular interactions are some of the multiple examples. ${ }^{4,9}$

Self-organization has been studied strongly in view of the design of nature-like materials. This process, defined as a spontaneous but controlled formation of architectures by cooperative non-covalent interactions, plays a fundamental role in biomechanisms and is one of the main reasons for evolution of matter. ${ }^{5,10-11}$ From protein recognition to storage of information as well as muscle contraction, all these natural processes rely on self-assembly. Intermolecular forces and non-covalent interactions are strongly involved in the formation of complex hierarchies, and provide those with a dynamic behavior. 5, 9, 12 Hydrogen-bonding is especially of particular 
importance in supramolecular systems due to its directionality. These physical bonds can be found in multiple biological components, and one of the most important examples is the stabilization of deoxyribonucleic acid (DNA) helices. ${ }^{9}, 12$ However, the full comprehension and replication of supramolecular biosystems is still a challenge and crucial for the development of smart devices with required properties like compatibility, multifunctionality, responsiveness, and degradability.1, 6 A controlled non-covalent selfassembly in bottom-up approaches would not only lead to an adaptive behavior, but would also reduce structural defects of biomaterials. ${ }^{13}$ Additionally, the utilization of biomacromolecules such as proteins in nanomaterial design, would provide many advantages in view of molecular recognition and replication of natural mechanisms.

The aim of this work was therefore, the development of an innovative and simple approach to produce novel supramolecular microgels for bio-applications. Currently, there is only a minor number of non-covalent crosslinked microgels known in literature, and their synthesis implicates multi-step procedures, starting from the design of special precursors. Contrary to this, the synthesis technique established in this thesis combines the advantages of a polymerization and self-assembly in a one-step method. Herein, the bioactive natural polyphenol TA was proposed as supramolecular crosslinker for the synthesis of PVCL-based microgels due to its interesting ability to form hydrogen bonds with polymeric materials. Moreover, the microgels should be designed as responsive and degradable carriers for the transport of active compounds. This method should offer a facile opportunity for the incorporation of biological macromolecules and bioactive compounds into multifunctional supramolecular and switchable polymer-based systems.

\subsection{State of the Art}

\subsubsection{Polymer-based Biomaterials}

In our days, the prefix "bio" has become of high significance in research and development in view of life quality. Therefore, the concept of Biomaterials has been arousing enormous interest lately. There are approx. 75.000 scientific articles related to this topic currently, and almost $70 \%$ has been published in the last 13 years. The first articles using this concept are from the 60's and since that time the definition has evolved more and more. ${ }^{14-15}$ Today, materials are defined as Biomaterials, if they are 
designed for biomedical applications and come in contact with bioorganisms for the performance of their functions. In this context, innumerable components have been developed, and depending on their derivation, they have been classified in three main groups: synthetic, nature-derived and hybrid materials. 2-3, 16 Independent of the material type, their production implies several efforts due to the large number of factors that have to be considered in view of biological application. Besides the production and costs, the clinical requirements are crucial. Not only properties like morphology, stiffness, strength and elasticity, but also the chemical composition and stability are included in biomaterial design. Especially, toxicology and biocompatibility define the safety of the final product and are decisive characteristics for its utilization. ${ }^{16}$

Natural as well as synthetic polymers are promising components for the synthesis of medical biomaterials, due to their controllable mechanical properties as well as biocompatibility, biodegradability, and variable functionality. ${ }^{6}$ Proteins like collagen and fibrin, polysaccharides and DNA are some examples of natural polymers used in biomaterials. ${ }^{16-18}$ However, synthetic polymers have been studied strongly in the biomedical area due to their controlled polymerization techniques and thus, their triggered and multifaceted properties. Nylon, poly(methyl methacrylate) (PMMA), poly(2-hydroxyethylmethacrylate) (PHEMA) and are few examples of polymers that have been utilized in medicine since $1940.2,4,18$ In terms of tissue engineering, each organ and its functionality have to be considered specifically for the selection of the biomaterial. The cell interaction with the medical device and their proliferation depends on its structure, stability, compatibility and consistence. ${ }^{2,19-20}$ In case of partial or total tissue damage, the replacement of the affected organs with synthetic or natural substitutes is required during the medical treatments. Normally, the employment of implants generates inflammatory reactions as a response against the foreign body. Protein adsorption, cell adhesion, fibrosis, and the formation of foreign body giant cells (FBGCs) on the implant surface lead to an inappropriate functionality of the device that implies further interventions. ${ }^{21}$ Moreover, mechanical stability and resistance are further relevant factors. In orthopedics, Polylactic acid (PLA), poly(lactic-co-glycolic acid) (PLGA) and polycaprolactone (PCL) are nontoxic and biodegradable synthetic polymers that have been studied many years for this kind medical materials. ${ }^{4,18,22-23}$ Their biocompatibility plays a primary role to avoid problems such as implant rejection 
or pathogen transfer. ${ }^{4,24}$ In ophthalmological material design, light transmission, tearfilm wettability, oxygen permeability and protein resistance are important requirements that have to be fulfilled. 2-hydroxyethylmethacrylate (HEMA), methyl methacrylate (MMA), as well as ethyl acrylate (EA) and methacrylic acid (MAA) are some examples of monomers used in this field for the production of sensitive hydrogels. ${ }^{2,20}$ In the case of drug transport, the synthesis of "smart" materials would help to achieve challenges like a targeted release, reducing the need of regular medication treatments and improving the preservation of medicine.25-26 Hereby, responsive polymers present new options for controlled drug delivery. Poly( $N$-vinyllactams) are amphiphilic, thermosensitive and show and low toxicity profiles. ${ }^{27-29}$ Their amide group is not only the reason for most of these properties, but is also of high relevance in nature. Therefore, these polymers have raised enormous attention in the bio-medical field. ${ }^{27-28}$ In the case of PVCL, the pharmaceutical interest increases additionally due to its proven biocompatibility and its lower critical solution temperature (LCST) close to the body temperature $\left(37^{\circ} \mathrm{C}\right) .{ }^{28-31}$ Furthermore, its modification with other biodegradable and bioactive components enables the synthesis of multifunctional and adaptive systems. ${ }^{6,32-33} \mathrm{~A}$ broad variety of copolymers as well as gels and films have been produced and studied as stimuliresponsive systems. ${ }^{27,31}$

\subsubsection{Colloidal Polymeric Systems: Microgels}

Among the extended list of new concepts that have been developed in biomaterials and molecular engineering, hydrogels are one of the most studied currently. These polymeric networks consist of a liquid and a solid phase, and have the special ability to swell strongly in water. The gel formation is based on physical or chemical crosslinking processes between the polymer chains, leading to 3-dimensional structures. The degree of swelling, and thus, the water uptake capacity depends mostly on the polymer type and its functionalities, which are responsible for the interactions with the aqueous medium. 19, 26, 34 Depending on their composition, but also structure and size, hydrogels can be classified into different groups. Colloidal hydrogels with a dimension from $1 \mathrm{~nm}$ up to $1000 \mathrm{~nm}$ are categorized as microgels. ${ }^{35-36}$ These spherical nano- or microsized particles are synthesized generally by radical polymerization methods. The most used approaches are currently precipitation and emulsion polymerization. High colloidal stability, well defined structure, high surface area and a low surface tension are some of 
the particular properties of microgels. However, their responsiveness towards external stimuli is the feature that distinguishes them from other colloids. ${ }^{32-33,37}$ Microgels show a sensitive behavior to environmental changes such as modification of the temperature, ${ }^{34,38-40} \mathrm{pH}^{41}$ magnetic field ${ }^{42}$ or light intensity. ${ }^{43}$ As a result, their degree of swelling can be varied in a controlled manner.6,19,38,44 This property arouses enormous interest in the utilization of microgels in the medical field. In combination with their high water content and their 3-dimensional structure, microgels can be used as carriers for the encapsulation of bioactive compounds, offering great advantages in targeted drug delivery. ${ }^{32,45-46}$

Different monomer types can be used and combined not only to obtain sensitive characteristics but also multi-responsive microgels. ${ }^{47}$ Especially, $N$-isopropylacrylamide (NIPAAm) and $\mathrm{N}$-vinylcaprolactam (VCL) have been studied intensively for the synthesis of thermoresponsive microgels. However, PVCL-microgels have gained particular attention owing their proven biocompatibility. ${ }^{31}$ Due to their volume phase transition temperature (VPTT) at approximately $32{ }^{\circ} \mathrm{C}$ close to the body temperature, VCL-based microgels are considered potential candidates in the drug delivery field. ${ }^{30-34}$ At temperatures below the VPTT, the microgel network shows a hydrophilic behavior. The polymer chains interact strongly with the water molecules, and a swollen state can be achieved. If the temperature increases above $32{ }^{\circ} \mathrm{C}$, the polymer-to-polymer interactions become stronger, leading to a the collapse of the microgel and the release of the solvent molecules in a controlled manner (Figure 1). ${ }^{32-33}$

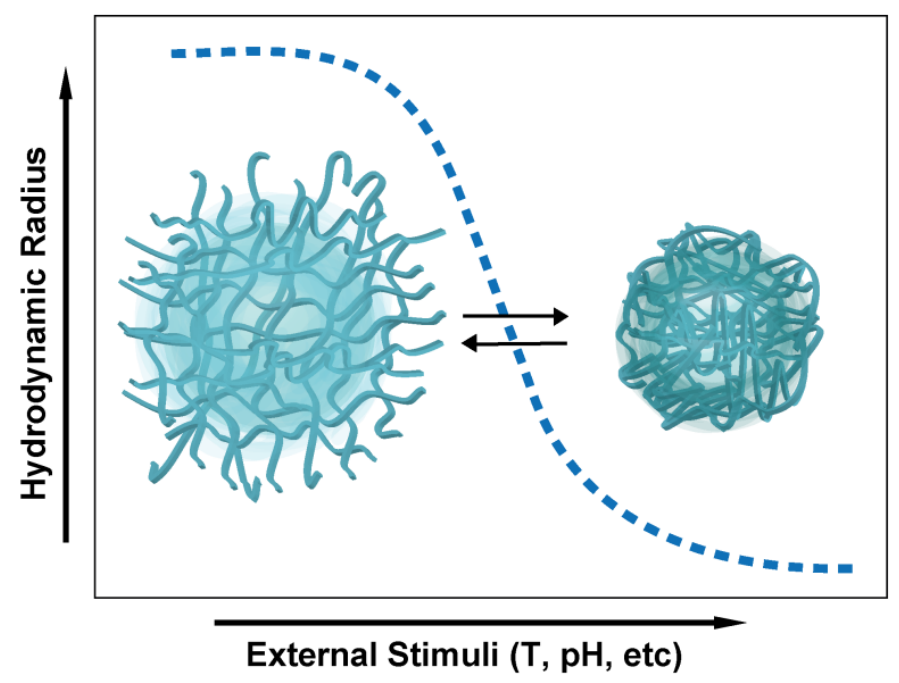

Figure 1. Graphical representation of the microgels response to external stimuli. 
This excellent property in combination with their water solubility and colloidal stability make microgels suitable candidates as systems for controlled drug release. In addition, their modification possibilities by microgel functionalization offer novel possibilities for the incorporation of hydrophobic building blocks within the polymeric network, which can serve as containers for the encapsulation of pharmaceutical substances. ${ }^{33,48-50}$

In microgel design, the monomer type is not the only factor that plays an important role. The selection of a suitable crosslinker is decisive in terms of material density, stability and degradability. Normally, microgels are crosslinked by covalent bonds. ${ }^{32-33}$ This is nevertheless, a disadvantage in view of degradability. ${ }^{51}$ Agents with cleavable groups (disulfide or ester) have been utilized for the synthesis of degradable microgels. ${ }^{48,52}$ However, this leads to longer degradation rates. Actually, only a small number of physical crosslinked microgels have been developed. In 2016, our research group reported a novel approach to produce microgels crosslinked by supramolecular hostguest complexes between $\beta$-cyclodextrin and cholesterol. ${ }^{53}$ Song et. al. published also recently the synthesis of supramolecular microgels based on supramonomers. ${ }^{51}$ However, in the known cases, the microgel fabrication implies complex procedures and multi-step syntheses of precursors. ${ }^{51,53-56}$ A controlled design of supramolecular microgels, as well as controlled microgel disintegration are still challenges in colloidal science.

\subsubsection{Supramolecular Nanomaterials}

Supramolecular chemistry, well known as "the chemistry beyond the molecule" describes processes that involve physical interactions. 3,36,57 Most natural mechanisms are based on a spontaneous association of molecular segments from a less ordered to a more ordered state by weak and non-covalent interactions. Herein, the balance between attractive and repulsive interactions as well as its reversibility plays an enormous role.4, 10-11, 58

Researchers have thus been studying intensively different natural as well as synthetic self-assembled systems. In view of precise medicine and targeted drug delivery, supramolecular materials exhibit excellent properties in terms of selectivity, molecular recognition, and self-healing among further features. ${ }^{58}$ Moreover, supramolecular organization can be explored as a hierarchical process not only at molecular scale, but 
also at mesoscopic and macroscopic ranges. In comparison to classical medical materials, supramolecular design promotes strongly the desired control of material properties in a bio-inspired way. 1,13,58 Figure 2 exhibits schematically examples of main non-covalent forces. Host-guest interactions have been developed mostly in view of supramolecular delivery systems, however, hydrogen bonding itself arouses particular interest due to its directionality and significance in natural systems. ${ }^{3,9}$ This non-covalent force describes the attraction between a covalently bound hydrogen atom (donor) and a more electronegative atom (from another molecule or molecular segment) with a lone pair of electrons (acceptor).3, 12, 59 Even though each single interaction is fragile, cooperative effects and the association mechanism increase the binding strength and stability ${ }^{57}$ Relevant functions of hydrogen bonds in nature are, for example, structural specifications and recognition of proteins and nucleic acids. For instance, base paring of DNA and ribonucleic acid (RNA) relies on hydrogen bonding. ${ }^{12,}$ 60-61
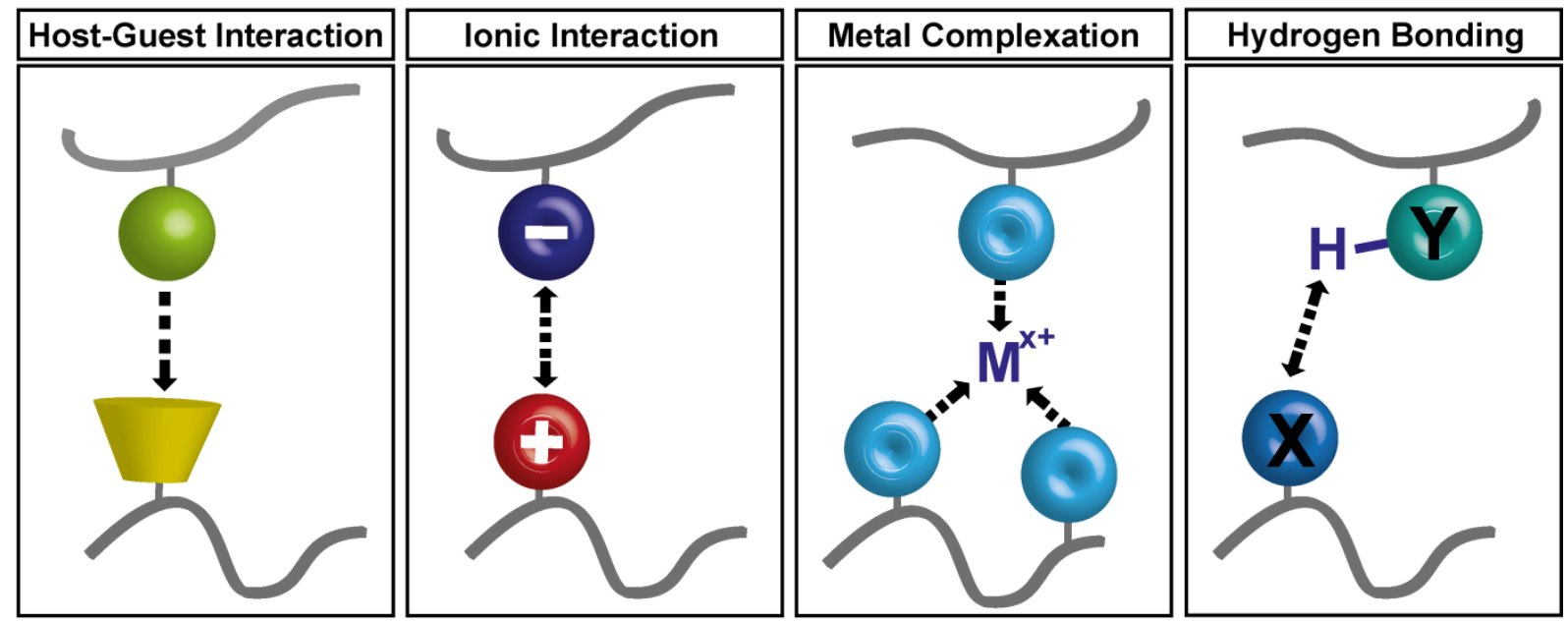

Figure 2. Schematic representation of various non-covalent interactions.

As highlighted previously, medical science has been focused on the usage of polymeric materials as interesting candidates for clinical treatments. Besides the 3-dimensional porous structure and high water content, in particular, hydrogels with supramolecular origin offer particular adaptability. Crosslinking mechanisms based on host-guestinteractions, ionic forces, metal-ligand-complexation and/or hydrogen bonds are applied in these cases. ${ }^{3}$ Comparable to covalent crosslinked hydrogels, the mechanical properties can be influenced by the type of crosslinking and the network density. Nonetheless, the dynamic nature based on the energetic equilibrium between association and dissociation leads to a reversible assembly. The modularity, mechanical 
tunability, responsiveness and biomimicry of such polymeric biomaterials propose a novel pathway to overcome actual challenges like incompatibility and undesired biological responses (e.g. inflammations, implant rejection, etc.) 3, 57 Targeted material assembly without structural defects, in addition to the molecular selectivity and reversible behavior are also important parameters to produce novel nanocarriers that can respond accurately to the physiologic requirements. ${ }^{62}$ Depending on the active compound, drug solubility, long-term conservation, and specific drug localization are still current complications in drug delivery. Hence, supramolecular drug delivery systems offer an interesting solution to overcome these challenges. Furthermore, their modular behavior extends the possibility to create multi-compartment systems for drug combination, kinetically controlled delivery, and patient-specific treatments. A full comprehension of their assembly mechanisms, as well as studies of their interactions invivo are thus indispensable requirements for their utilization in clinical approaches. ${ }^{62-63}$

\subsubsection{Bioactive Polyphenols}

As emphasized earlier, modern clinical treatments require the design of adaptive bio-inspired devices. Still, these approaches are of high complexity, due to the large number of challenges that have to be overcome such as implant rejection, pathogen transfer, or side effects. Therefore, factors like controlled release, gaseous exchange, prevention of secondary infections, as well as uncomplicated removal or triggered material degradation are necessary to obtain the best therapeutic results. ${ }^{24-25,64}$ Biomimicry is to this effect, an indispensable research field to learn from nature how to solve all these current medical issues in a targeted and energy-saving way, without further negative effects. Therefore, utilization of bioactive natural compounds is a great option in biomaterial design.

Among the broad list of interesting components in nature, polyphenols have aroused great interest since many centuries. Whether in green tea, wine, coffee or chocolate, polyphenols can be found in several natural food products, and have been related strongly to human health and longevity. ${ }^{65-66}$ Agrimony, tree peony, bearberries, and hawthorn are just few examples of polyphenol containing herbs that have been applied in traditional medicine all over different cultures and times. ${ }^{67-69}$ Already in the archaic period, these substances were of great importance for leather treatments due to their 
tanning effect, reason why they were classified as tannins. ${ }^{66,70}$ Since then, the definition of polyphenols has been modified strongly according to the discoveries of their infinite properties. The current definition, also known as White-Bate-Smith-Swain-Haslam (WBSSH) definition, is based on the crucial investigations of 4 renowned (bio- and organo-) chemists from the last century: Theodore White, Edgar Charles Bate-Smith, Tony Swain, and Edwin Haslam. Their studies lead to the classification of polyphenols as water soluble secondary metabolites from vegetable sources, with molecular weights between 500 to $4000 \mathrm{Da}$, and a polyphenolic character. Their structure based on 12 to 16 phenolic hydroxyl groups and 5 to 7 aromatics per 1000 relative molecular mass, is responsible for their main features such as intermolecular and metal complexation, as well as protein und alkaloid precipitation.66, 68-69, 71 Due to the large number of compounds that meet these main characteristics, polyphenols have been classified in 3 further subgroups, depending on their derivation. There are gallo- and ellagitannins (also known as hydrolyzable tannins, derived from gallic acid), proanthocyanidins (also known as condensed tannins, derived from flavan-3-ol units such as catechin), and phlorotannins (found in brown algae, derived from phloroglucinol). $66,68-69,71$

Throughout the last decades, more and more research groups have confirmed remarkable properties of polyphenols as antioxidant, antibacterial, antimutagenic, and antitumor substances. ${ }^{72-75}$ It has been suggested that their antioxidant activity supports the prevention of aging diseases and degeneration, as well as cardiovascular syndromes. ${ }^{76-77}$ Polyphenol-rich food products like green tea and wine have thus been proposed as vital part of the daily nutrition. As an example, Giampieri et. al. published lately a review about strawberries as health promoters. This type of fruit has shown a high content of bioactive compounds such as flavonoids, anthocyanins and phenolic acids, among others. In their review, the authors summarized latest investigations related to in-vivo studies of the detoxifying effect of strawberry phenolic substances, as well as cell proliferation, DNA protection and repair. Moreover, they compiled different proposed mechanisms to explain the effect of polyphenols against oxidative diseases like cancer. ${ }^{78}$ It is important to remark that although such great advances have been made, the benefits of polyphenols in human health have not been completely confirmed and are still under discussion in terms of toxicity.66, 68, 71, 79 Nonetheless, their further 
features like their complexation ability enables their usage as building blocks for the creation of supramolecular biomaterials..$^{80-87}$

\subsubsection{Tannic Acid: Structure and Properties}

TA is one of the most studied gallotannins that has been used in biomaterial design. The chemical structure $\left(\mathrm{C}_{76} \mathrm{H}_{52} \mathrm{O}_{46}, 1701.19 \mathrm{~g} \cdot \mathrm{mol}^{-1}\right)$ of this polyphenol derives from gallic acid (GA) and is composed of a D-glucose center and 10 galloyl (phenolic) substituents (Figure 3).

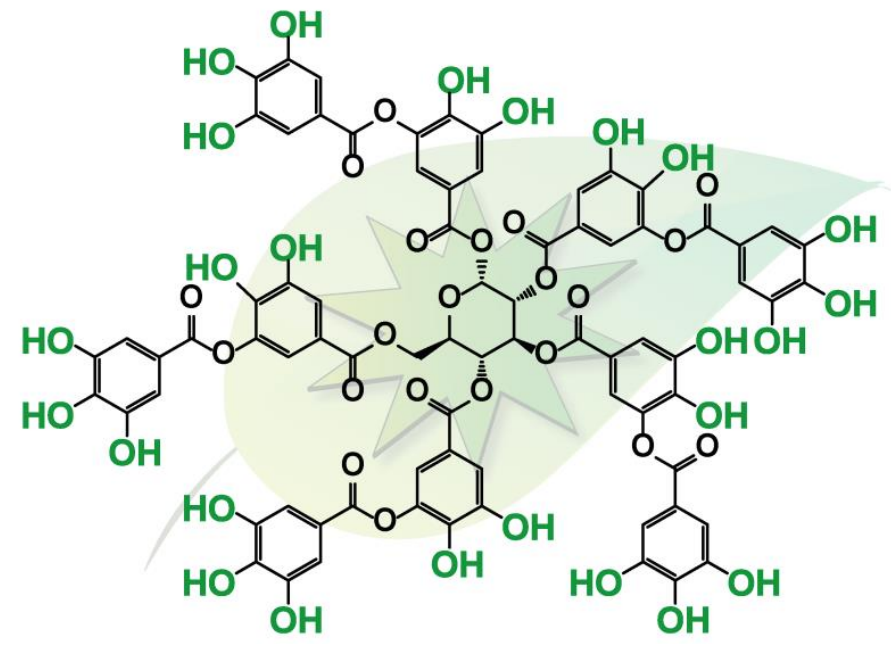

Figure 3. Chemical structure of the natural polyphenolic compound TA.

Its extraction can be performed from different vegetable sources such as Tara Spinosa, nutgall trees (Rhus Semialata), Sicilian sumac (Rhus Coriaria L), and Aleppo oaks. However; its separation from other gallic derivatives requires a complex procedure and therefore the term "tannic acid" is often used to describe a mixture of various gallic compounds. ${ }^{88}$

Its significance in science is highlighted in literature since the $19^{\text {th }}$ century, even though by that time, its main application was in leather manufacturing. The clinical utilization of TA was published firstly in the 1920's. ${ }^{15,} 89$ Herein, the polyphenol was analyzed as active compound for skin regeneration and treatment of burns. The interest of using tannic acid in medicine increased rapidly and further promising usages were discovered in relation to its antioxidant and complexation properties. ${ }^{73,81-83,90-92}$ So far, there have been innumerable research groups studying the antibacterial, antienzymatic, antihistamine, and antimutagenic features of this water soluble macromolecule, and all 
of these findings contribute strongly to its persistent importance within the biomedical field. $66,72,74,88,93-95$ In addition, the effects of tannic acid on human health and the influence of its dosage is also a critical point of study. ${ }^{93}$

In terms of biomaterial science, TA has been intensively studied in view of drug delivery, anticancer treatments, and synthesis of antibacterial, protein resistant and self-healing materials. ${ }^{72,84-87,94,96}$ Due to the multiphenolic structure, it interacts strongly with a large number of molecules and polymers by hydrogen bonding. Therefore, TA offers a vast number of options in view of self-assembly, molecular recognition and supramolecular chemistry. In 2008, the research group of Sukhishvili published a method to produce pH-responsive multilayers based on hydrogen bonded neutral polymers and TA. ${ }^{74}$ Moreover, Kozlovskaya et. al. synthesized successfully highly permeable and cytocompatible polymer/TA coatings for cell surface engineering. In their study, they demonstrated long-term preservation of the cell functions and survivability ${ }^{97}$ Other studies have confirmed enzyme-responsive properties of TA. ${ }^{98}$ In context to protein complexation and enzymatic inhibition, TA has been suggested as a promising candidate against skin aging, Alzheimer disease as well as used for specific protein immobilization. ${ }^{77,99-100}$ Furthermore, different TA-based films and nanoparticles have been studied for responsive wound healing and targeted drug delivery. ${ }^{84-85,101-104}$ Park et. al. published recently an interesting review about chemical encapsulation of individual cells. Herein, they compiled latest studies about the utilization of phenolicbased shells as a promising alternative for cell coating and protection. ${ }^{103}$ Finally, a recent research work reported a facile method to produce TA-metallogels with self-healing properties. ${ }^{81}$ The ability of TA to complex metals is a straightforward way to integrate metal ions into the TA-based biomaterials, and provides a further option to design multifunctional supramolecular systems with self-healing properties. 


\subsection{0utline of the Thesis}

The principal objective of this $\mathrm{PhD}$ thesis was the development of a facile method to synthesize novel supramolecular microgels as degradable and stimuli-responsive carriers for the transport of active compounds (Figure 4).

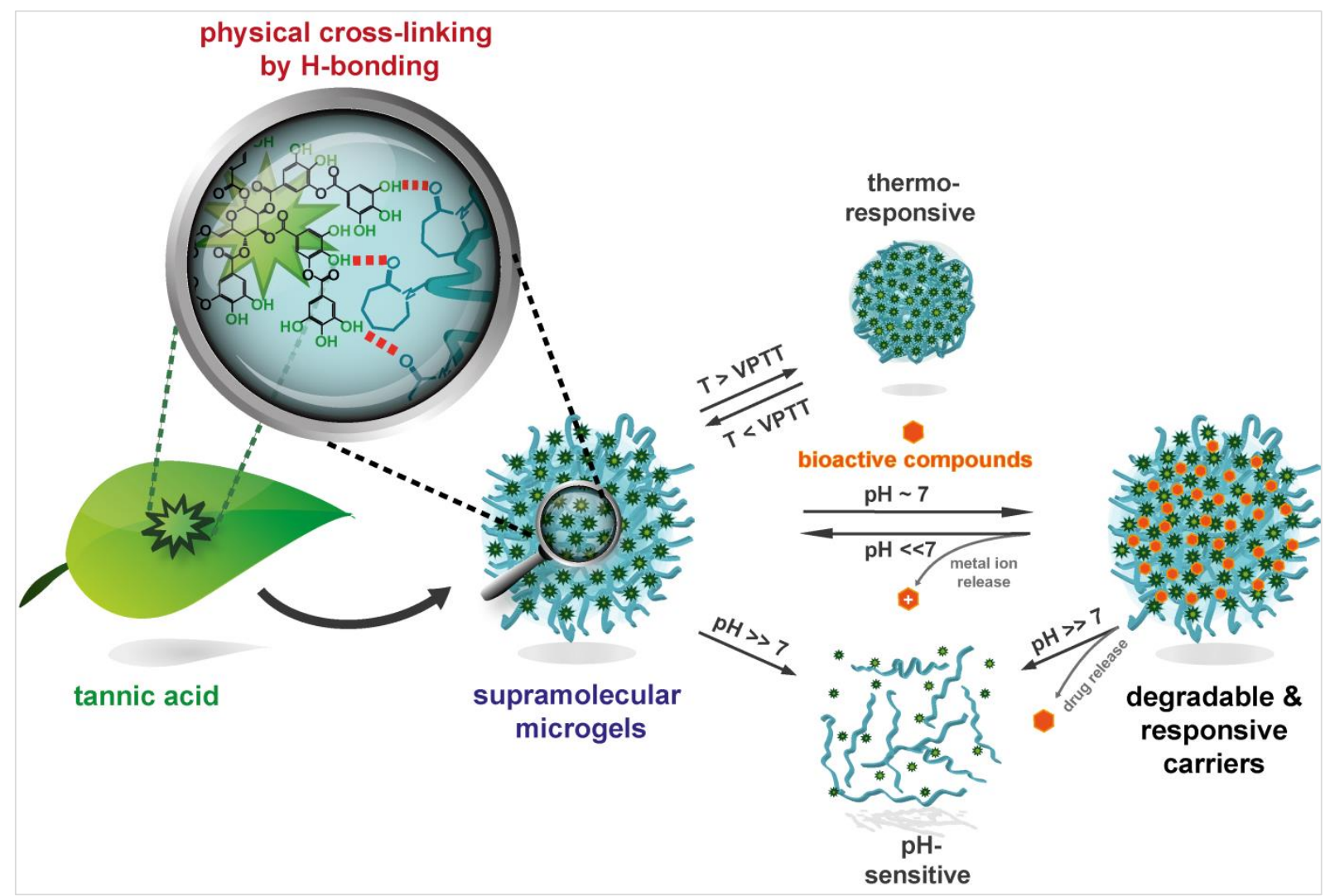

Figure 4. Scope of the thesis: Synthesis of bio-inspired supramolecular PVCL/TA-microgels and their utilization as degradable and stimuli-responsive carriers for bioactive compounds.

The scope of this work was divided in four main parts: 1.) synthesis and material characterization, 2.) system optimization, 3.) study of material disassembly and 4.) analysis of promising applications. In the first phase, preliminary investigations were carried out in view of proof of concept and microgel formation. Hereby, microgels based on PVCL were synthesized via radical precipitation polymerization in a batch approach using TA for the first time as an innovative non-covalent crosslinker. The stability of the produced microgel, as well as its physical and chemical properties were investigated by STEM, DLS and Raman spectroscopy among other characterization methods. Moreover, the influence of the crosslinker content was studied systematically and the supramolecular building was examined. In the second section, the yield and conversion 
in dependency of the time were analyzed, and the effect of tannic acid during the polymerization of VCL was determined. Based on this study, experiments in view of synthesis optimization were carried out and all obtained materials were characterized in terms of chemical and physical properties.

Due to the pH-sensibility of PVCL/TA-based materials, the third part of this thesis was related to the analysis of the $\mathrm{pH}$-triggered disassembly of the optimized supramolecular microgels. Besides this, the simultaneous release of TA was also studied. Finally in the last chapter, the investigations related to the utilization of the supramolecular PVCL/TAmicrogels as pH-sensitive colloidal carriers are presented. Herein, preliminary investigations were performed in view of promising applications of the novel supramolecular microgels in the biotechnological field. Different experiments about metal-ion transport as well as encapsulation of bioactive and antibacterial compounds were carried out using the developed synthesis approach. 


\section{Tannic Acid as Supramolecular Crosslinker for PVCL-Microgels Synthesized by Batch Precipitation Polymerization}

Sections of this chapter have been published in a modified form as a Communication in Macromolecular Rapid Communications.

C. Molano López, A. Pich: Supramolecular Stimuli-Responsive Microgels Crosslinked by Tannic Acid, Macromol. Rapid Commun. 2018, 39, 1700808. Copyright Wiley-VCH Verlag GmbH \& Co. KGaA, Weinheim. Reproduced with permission.

\subsection{Introduction}

One of the fundamental concepts in natural science related to the origin and evolution of living species is self-organization. This spontaneous but temporal and spatial controlled hierarchical assembly is desired in view of biomimicry to achieve a programmed formation of materials with adaptive features and responsive properties. Herein, molecules or molecular segments reach a more ordered state by non-covalent interactions, and the balance between these attractive and repulsive forces as well as their stability play a primary role in many different biological functions such as molecular recognition, simultaneous coordination, transmission and storage of information. 1, 5, 10-11 1,13,58 The application of self-assembly mechanisms offers therefore great possibilities in terms of controlled material design and the reduction of structural defects because of the high selectivity of supramolecular interactions. ${ }^{9-11}$

Non-covalent interactions describe electrostatic attractions between atoms or molecular segments, induced by their electric multipoles. Even though these forces are classified as weak interactions, the formation of stable structures results from their cooperative effect. ${ }^{105}$ Van der Waals forces, ion pairs, $\pi$-interactions and hydrogen bonds are some of the most occurring physical interactions. ${ }^{5}, 10-12,106$ In terms of selectivity, the directionality in hydrogen bonding is a key aspect, particularly in supramolecular design. ${ }^{12}$ There are a large number of components in nature that can interact with further substances by hydrogen bonding. Polyphenols and especially TA, are important metabolic substances with strong complexation characteristics. ${ }^{66,68}$ Over the last years, different research groups have confirmed the significance of these macromolecules in human health and used them in view of drug delivery, anticancer treatments, and 
synthesis of antibacterial, protein resistant and self-healing materials. For instance, TA has been used for supramolecular design. Due to the fact that this molecule serves as multiple hydrogen donor, it offers a broad variety of possibilities in view of self-assembly. Its incorporation during the synthesis of biomaterials in combination with its versatile properties leads to a broad variety of applications in medicine and biotechnology. $72-73,85,93,107$

\subsection{Results and Discussion}

\subsubsection{Proof of Concept: TA Interactions}

Inspired by the previous discoveries and the scientific ambition to mimic nature-like properties using biomolecules in design of "green materials", the principal objective of this thesis was the production of supramolecular VCL-based microgels using a bioactive component. Herein and contrary to the common approaches, TA was studied for the first time as innovative and natural physical crosslinker. In this chapter, the preliminary studies were performed in view of interactions between PVCL and TA, and the microgel formation.

Interactions between PVCL and TA:

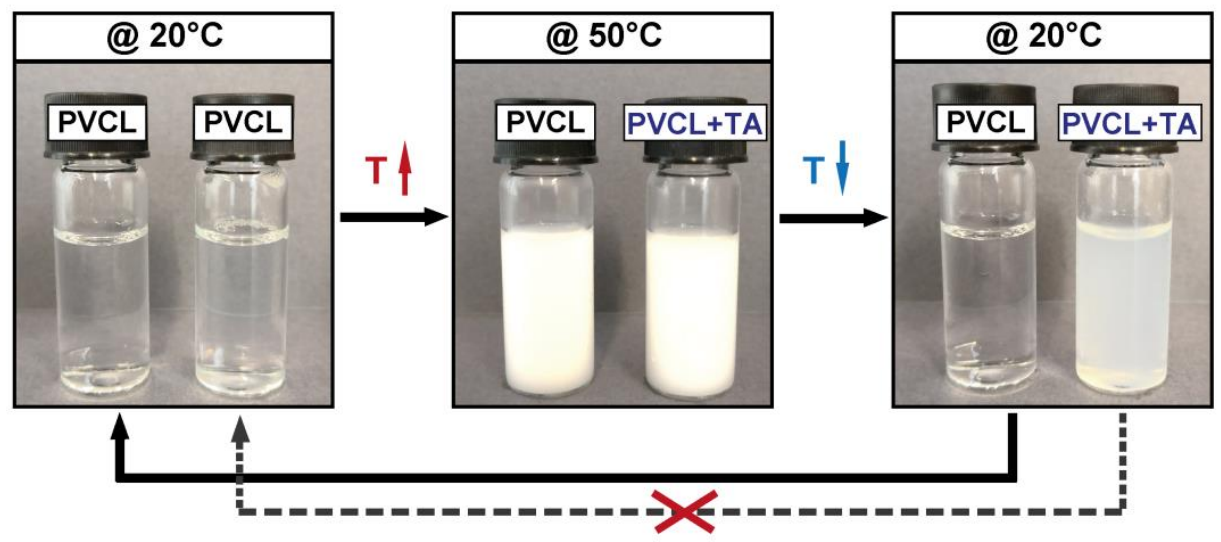

Figure 5. Prior test to determine the formation of bonds between PVCL linear chains and tannic acid.

As starting point and before the microgel synthesis, different proof-of-concept tests were performed to confirm the ability of TA to interact with polymeric compounds and other substances. Firstly, a basic experiment was carried out to determine the formation of stable PVCL/TA-structures. Figure 5 displays the resulting observations. Aqueous solutions (reference: left vial, sample: right vial) of polymeric linear chains (PVCL) were 
prepared at room temperature (@20 $\mathrm{C}$ ). Subsequently, the solutions were heated up to $50{ }^{\circ} \mathrm{C}$. As mentioned previously, PVCL is an amphiphilic polymer and therefore water soluble. Nevertheless, this material shows a responsive thermal behavior. Therefore, its solubility is temperature dependent. At $20^{\circ} \mathrm{C}$, the polymeric chains are hydrophilic, and in a relaxed state. The solutions are thus clear. By increasing the temperature over the LCST of the polymer, PVCL undergoes transition to the collapsed state forming compact globuli. This leads to a strong increase in the turbidity of the PVCL solution. At this point (@50 ${ }^{\circ} \mathrm{C}$ ), TA was added to one of the solutions (right bottle). Finally, both; the reference and the TA-containing sample, were cooled down to room temperature. In the case of the reference, the solution became transparent again pointing out the relaxation of the polymer chains, and thus, the reversibility of its thermoresponsiveness. Contrary to this effect, the sample containing TA remained turbid after cooling down. This indicates the presence of agglomerates and confirms the strong and stable interactions between PVCL and tannic acid.

In addition to this initial investigation, the interactions between the polymer chains and the polyphenol were characterized by isothermal titration calorimetry (ITC) to obtain preliminary information about the thermodynamic parameters of the physical interaction (Figure 6).

a.) ITC-measurement of PVCL and TA:

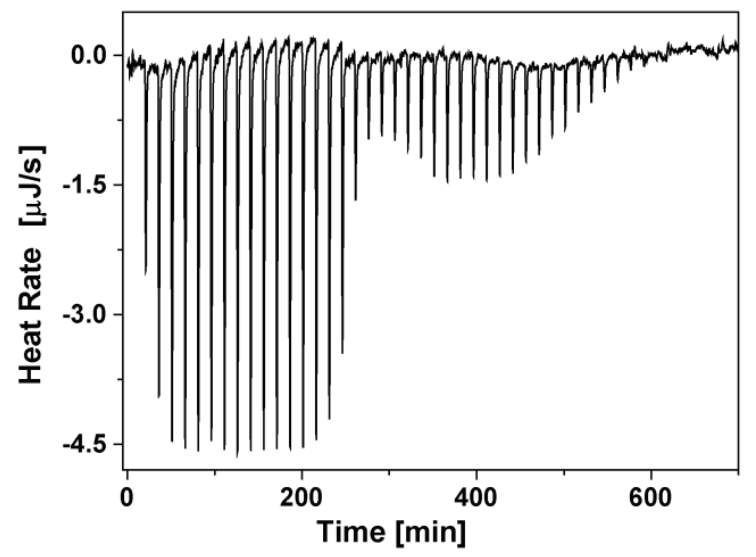

b.) ITC-data analysis:

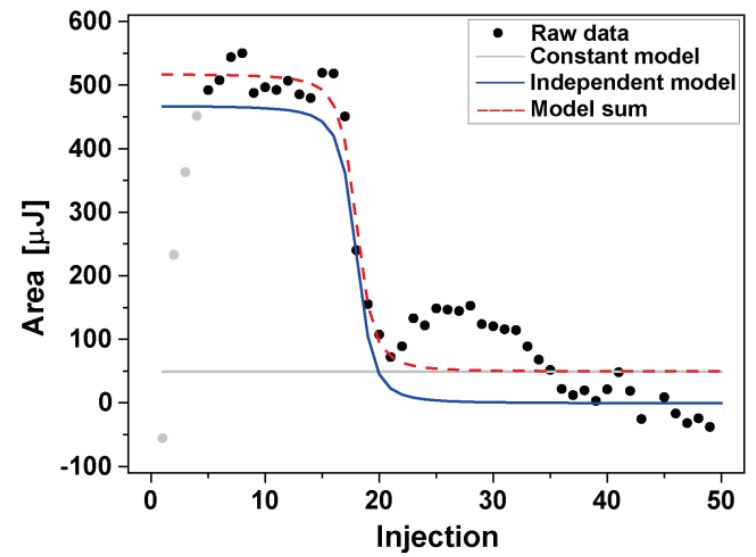

Figure 6. Preliminary thermodynamic characterization of the interactions between linear PVCL $\left(\mathrm{M}_{\mathrm{n}}: 60 \mathrm{~kg} \cdot \mathrm{mol}^{-1}\right)$ and TA via ITC.

During this experiment, linear chains of PVCL with a defined molecular weight were titrated with TA. Therefore, a specific volume of the polyphenol was injected to the sample cell in $5 \mathrm{~min}$ intervals. The heat rate of the reaction was then monitored in 
comparison to a reference of pure PVCL. Figure 6a displays the obtained diagram. Herein, one observes firstly that the heat rate trend shows notably the complexity of the binding process between the PVCL and TA molecules. During the 3 first injections ( $\mathrm{t}: 0-45 \mathrm{~min}$ ), the signal intensity increases slowly and the most negative heat rate is not reached before $60 \mathrm{~min}$. Compared to this, in a simplified one-to-one binding system, the maximum would be expected by the first injection. Still, after the maximal heat rate is obtained, the measured data display the expected decrease of the peak intensity. A fall of the signal strength normally corresponds to the finalization of the reaction between the reactants. However the intensity increases unexpectedly once more in the time frame between 300 and $600 \mathrm{~min}$ indicating further interactions. These primary observations could be assumed as cooperative mechanisms or steric rearrangements. Herein, it is important to consider the complexity of both molecular structures; PVCL-chains and TA, especially in the case of the polyphenolic compound, since it shows high multifunctionality. Its structure could imply steric hindrance, meaning that not all the hydroxyl-groups are available at the same time and cannot interact with the carbonyl groups of PVCL simultaneously. Moreover, the length of the polymeric chains and the number of their repeating units play a significant role. Their mobility in aqueous media can also affect the accessibility and interaction ability to TA.

Despite the suggested complexity of the reaction, a combination of two simplified models (constant and independent, NanoAnalyze software, TA Instruments) was used exemplarily for the data analysis as a reference model to obtain a preliminary knowledge about the binding process. By integration of the measured signals and determination of the peak area, the data could be fitted and analyzed in terms of thermodynamics (Figure 6b). The ITC-calculated thermodynamic parameters are summarized in Table 1.

At first, a data blank of $50 \mu \mathrm{J}$ was calculated by the constant model. Moreover, the independent model applied to the ITC assay provides a complete thermodynamic profile of the interaction of the free molecules in solution, including: binding stoichiometry $(n)$, binding enthalpy $(\Delta \mathrm{H})$, entropy $(\Delta \mathrm{S})$, association constant $\left(\mathrm{K}_{\mathrm{a}}\right)$, and dissociation constant $\left(\mathrm{K}_{\mathrm{d}}\right)$. Very interesting is the result obtained for the stoichiometric relation between PVCL and TA. Expected is a value of 0.04 since the theoretical ratio of each VCL repeating unit 
within the polymeric chain to the hydroxyl groups of one TA-molecule is $\mathrm{n}: \mathrm{VCL} / \mathrm{OH}(\mathrm{TA})=1 / 25=0.04$. Since each molecule of the polyphenol has 25 hydroxyl groups, this means that each VCL unit can bind to 0.04 of a TA molecule, forming a maximum of 25 hydrogen bonds per TA. By the ITC data analysis an average of $n: 0.034$ was calculated. This value reveals that nearly every carbonyl group of the PVCL chains interacts to a hydroxyl group of TA.

Table 1. Thermodynamic parameters obtained by ITC.

\begin{tabular}{|c|c|}
\hline \multicolumn{2}{|c|}{ Constant model } \\
\hline blank & $50 \mu \mathrm{J}$ \\
\hline \multicolumn{2}{|c|}{ Independent model } \\
\hline $\mathbf{n}$ & 0.034 \\
\hline $\boldsymbol{\Delta} \mathbf{H}$ & $93.59 \mathrm{~kJ} \cdot \mathrm{mol}^{-1}$ \\
\hline $\boldsymbol{\Delta} \mathbf{S}$ & $433.1 \mathrm{~J} \cdot \mathrm{mol}^{-1} \cdot \mathrm{K}^{-1}$ \\
\hline $\mathbf{K}_{\mathbf{a}}$ & $1.685 \cdot 10^{6} \mathrm{M}^{-1}$ \\
\hline $\mathbf{K}_{\mathbf{d}}$ & $5.936 \cdot 10^{-7} \mathrm{M}$ \\
\hline
\end{tabular}

Furthermore, an enthalpy of $\Delta \mathrm{H}: 93.59 \mathrm{~kJ} \cdot \mathrm{mol}^{-1}$ was determined by this preliminary study. This value corresponds to high bond strength and indicates the formation of strong hydrogen bonds. As a comparative example, water molecules show middle hydrogen bond strength in a range of 17 and $63 \mathrm{~kJ} \cdot \mathrm{mol}^{-1}$, depending on the temperature and pressure. ${ }^{108}$ By means of the calculated released enthalpy and the entropy changes $\Delta S$, the free Gibbs energy $(\Delta \mathrm{G})$ could be determined $(\Delta \mathrm{G}=\Delta \mathrm{H}-\mathrm{T} \cdot \Delta \mathrm{S})$. A value of $-35.5 \mathrm{~kJ} \cdot \mathrm{mol}^{-1}$ confirms that the equilibrium of the reaction is on the product site.

Finally, a high association constant of $\mathrm{K}_{\mathrm{a}}$ : $1.685 \cdot 10^{6} \mathrm{M}^{-1}$ was determined during this analysis. Herein, it is important to emphasize once more that this experiment was preliminary and the accuracy of the results has to be confirmed. Further investigations should be carried out, such as measurements of the interactions between VCL-monomer and TA, or linear PVCL-chains with various lengths. Of essential importance would be the design of a specific analysis model corresponding to the complexity of the system. However, this proof-of-principal-study supports the high strength and stability of the physical interactions between PVCL and the natural polyphenol. 

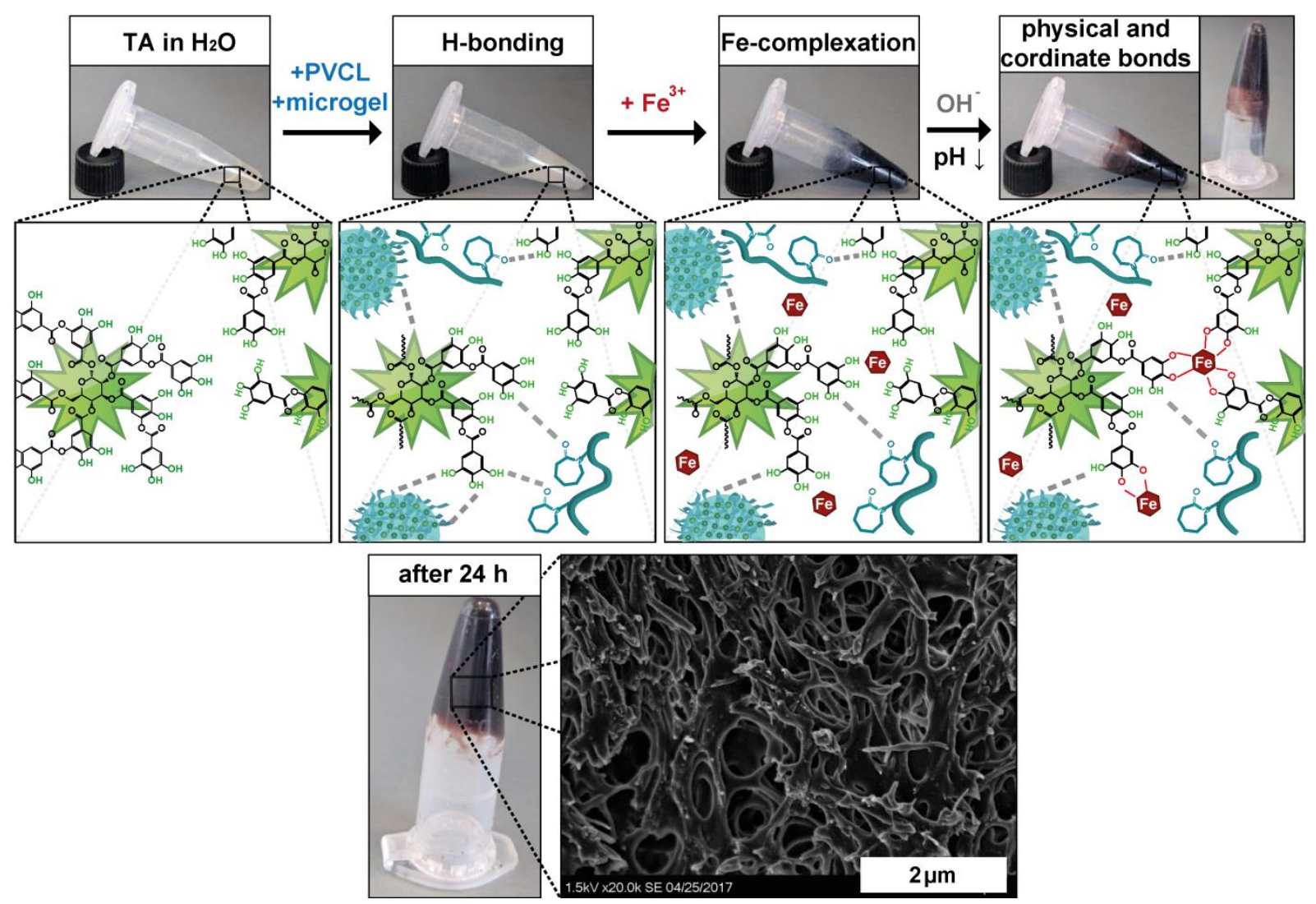

Figure 7. Formation of microgel/metal ion-based hydrogels

Finally, the complexation ability of TA to form stable bonds with metal ions was tested in order to proof this concept. Inspired by the latest reports of the Fan group and Carrusos research, a preliminary experiment was performed to produce supramolecular hydrogels based on a combination of various building blocks in different stages. Both scientific groups successfully produced different types of supramolecular hydrogels based on TA with water soluble polymers and/or metal ions. The supramolecular products showed interesting properties such as $\mathrm{pH}$-stimuli responsiveness, mechanical tenability, rapid self-healing and shape persistence, as well as adhesive strength. ${ }^{81-83}$

In the present work, the experiment was carried out in 4 steps (Figure 7). Firstly, pure TA was dissolved in water. Subsequently, an aqueous solution of free polymeric chains (PVCL) mixed with PVCL-based microgels was added for the formation of stable hydrogen bonds. In the third step, $\mathrm{FeCl}_{3}$ dissolved in water was added to the TA/polymer mixture. Finally, the complexation of the iron ions and the formation of coordinate bonds by decreasing the $\mathrm{pH}$-value (addition of $\mathrm{OH}^{-}$) took place. The images depicted in Figure 7 reveal the formation of stable hydrogels after $24 \mathrm{~h}$. The hydrogels 
showed flexibility and softness, adhesive behavior and high elasticity. Moreover, a second test was performed to analyze the hydrogel production using only $\mathrm{TA} / \mathrm{Fe}^{3+}$ complexes in combination with PVCL-based microgels (without free polymeric chains within the system). There was no gelation observed. This indicates that the density and relatively low flexibility of the microgels in comparison to free polymeric chains hinders the gel formation at a macroscale. Nevertheless, the presented analysis confirms the ability of TA to form complexes with metal ions and the stability of its interactions. In view of applications, these materials could offer a multifunctional hydrogel-network with controllable and multiple pore sizes which could be used as responsive containers.

In conclusion, the three different proof-of-concept studies revealed significant preliminary information about the reactive properties of the polyphenolic compound TA. On the basis of these confirmations, the study of the formation of physically crosslinked microgels took place.

\subsubsection{Preliminary Synthesis of PVCL/TA-Microgels}

1.) Addition of bio-active crosslinker TA $(t=0 s)$ :
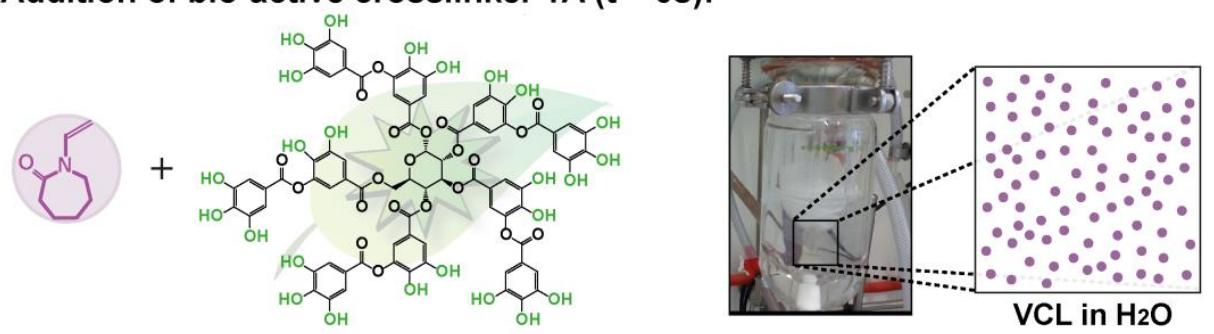

2.) Complexation between VCL and TA ( $t=1 \mathrm{~s})$ :
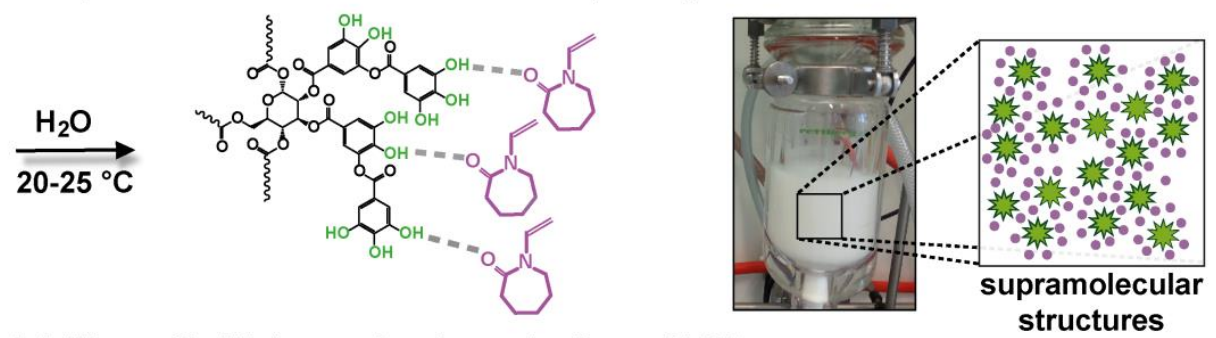

3.) Addition of initiator and polymerization of VCL:
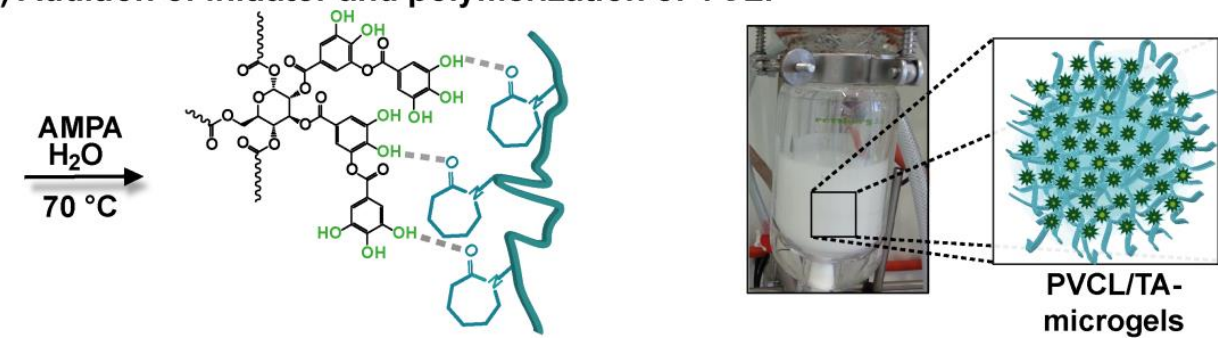

Figure 8. Scheme of the microgel synthesis. 
Herein, the synthesis of the PVCL/TA-microgels was carried out firstly by a modified batch precipitation polymerization. This approach includes three main steps (Figure 8). At the first stage, the crosslinker TA is added to the VCL solution at room temperature. Subsequently, the complexation between the monomer VCL with the TA molecules takes place as the second step. Due to the fact that tannic acid and the monomer VCL interact also by forming hydrogen bonds, the clear monomer solution becomes turbid after the TA-addition. This indicates the formation of supramolecular structures even before the polymerization is initiated. Finally, the reaction starts by the addition of a water-soluble initiator at $70{ }^{\circ} \mathrm{C}$. Hereby, the PVCL chains are formed in a precipitated state building microgel nuclei, while the strong interactions with TA lead to physical crosslinking and the formation of colloidal stable microgels.
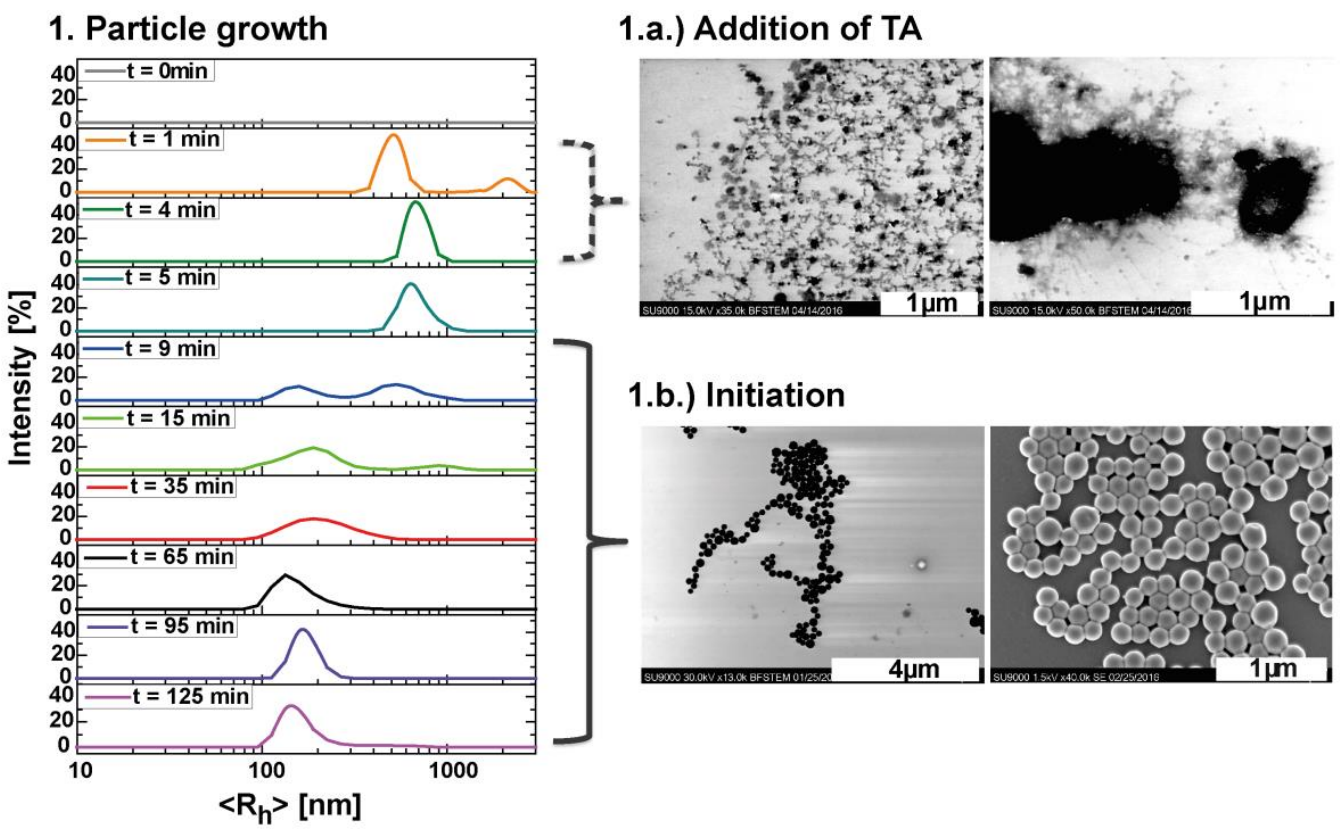

1.b.) Initiation

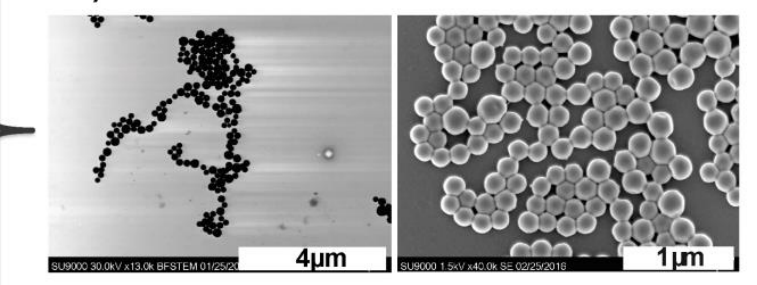

Figure 9. In-situ DLS measurement of $R_{h}$ during the polymerization (Sample: VCL/TA-0.5mol-\%) and corresponding 1.a.-b.) TEM and SEM images.

For a better understanding of the mechanism of the microgel synthesis, in-line dynamic light scattering (DLS) measurements and off-line scanning transmission electron microscopy (STEM)-analysis of the microgels were carried out at different reaction times. In Figure 9.1, the obtained particle size distributions for different reaction times are displayed. These results confirm the postulated hypothesis. After the addition of tannic acid $(\mathrm{t}=1-5 \mathrm{~min})$, the formation of large and polydisperse supramolecular structures can be observed. The undefined structures show hydrodynamic radii larger than $500 \mathrm{~nm}$, which decrease after the addition of the initiator. The in-line DLS spectra 
show that after 9 min a second signal appears in the range of 100 to $300 \mathrm{~nm}$. The signal intensity grows with the reaction time, while the peak at higher size values shrinks rapidly. After $30 \mathrm{~min}$, there is only one signal displayed, and after $60 \mathrm{~min}$ its size distribution becomes narrower. These results correlate with the microscopy-images depicted in Figure 9.1a and b. Hereby, it was concluded that the polymerization process is necessary for the microgel formation. The physical interactions between the monomer and the tannic acid are not sufficient to produce stable and well-defined particles. Polymer chains are needed to promote the microgel formation.

Additionally, the influence of various synthesis parameters was analyzed. Figure 10 compares the reaction solutions and products obtained by different parameter variations (surfactant amount and temperature). The images in Figure 10a represent the reference sample, which corresponds to the synthesis of PVCL/TA microgels reported above. The effect of the temperature and equilibration time was studied firstly. After the second step (complexation between VCL and TA), the reaction mixture is heated up to $70^{\circ} \mathrm{C}$, and the initiation of the polymerization follows after 5 min thermal equilibration. Herein, the equilibrating period was set up to $1 \mathrm{~h}$ in a second experiment as comparison (Figure 10b). The equilibration time shows an influence on the morphology of the microgels. The longer the time between heating up and initiation, the more irregular the particles become. Moreover, the decreased turbidity of the reaction solution suggests instability of the monomer in the presence of TA at higher temperatures. This hint was analyzed deeper, and the obtained results are presented in the following chapter.

Influence of temperature and surfactant on the microgel formation:

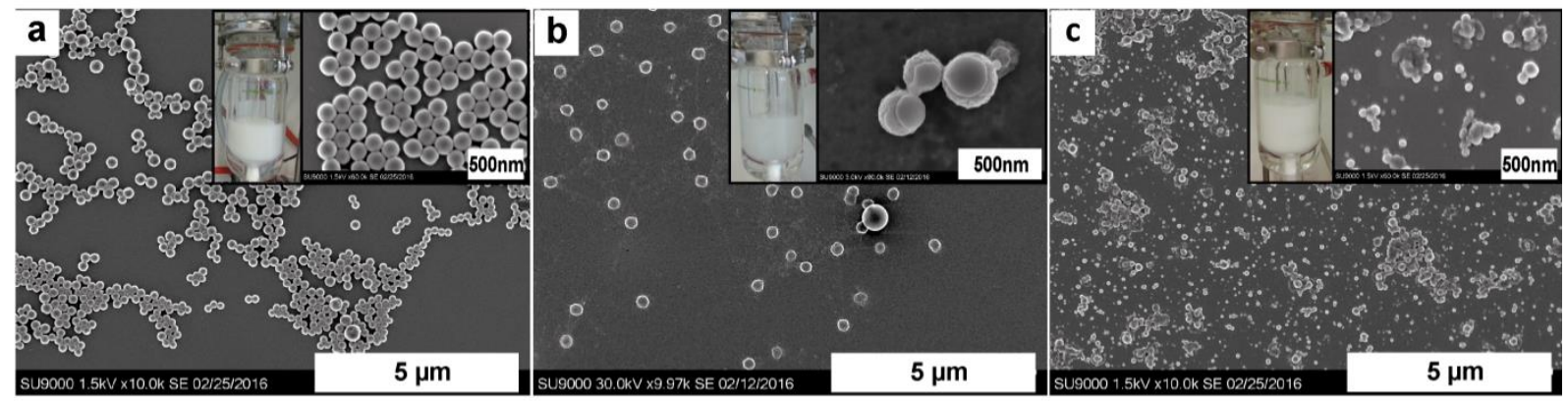

Figure 10. a) Reference: synthesis of PVCL/TA-microgels carried out as described previously. b) Effect of the temperature: modification of the second synthesis step. c) Effect of the sufactant: microgel synthesis without surfactant.

Furthermore, the effect of the surfactant was studied. In the before presented microgel synthesis, a cationic surfactant (Cetrimonium bromide, CTAB) was added to the 
monomer solution before the TA addition as stabilizing agent and for the control of the particle size..$^{32-33}$ In microgel design, the utilization of surfactants during the microgel formation is performed in view of particle stabilization. This technique helps to avoid aggregation effects, especially during precipitation polymerization. ${ }^{33}$ In this view, a third experiment without CTAB was performed. Herein, irregular and polydisperse microgels were obtained (Figure 10c). In conclusion to this approach, the results confirm that the equilibration time before the initiation has to be short and the utilization of surfactant is essential to obtain well-defined and monodisperse colloidal gels.

a.) Molecular structures:

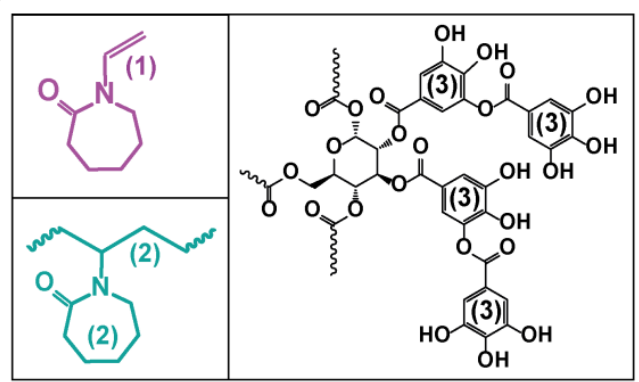

b.) Raman-spectra of the references:

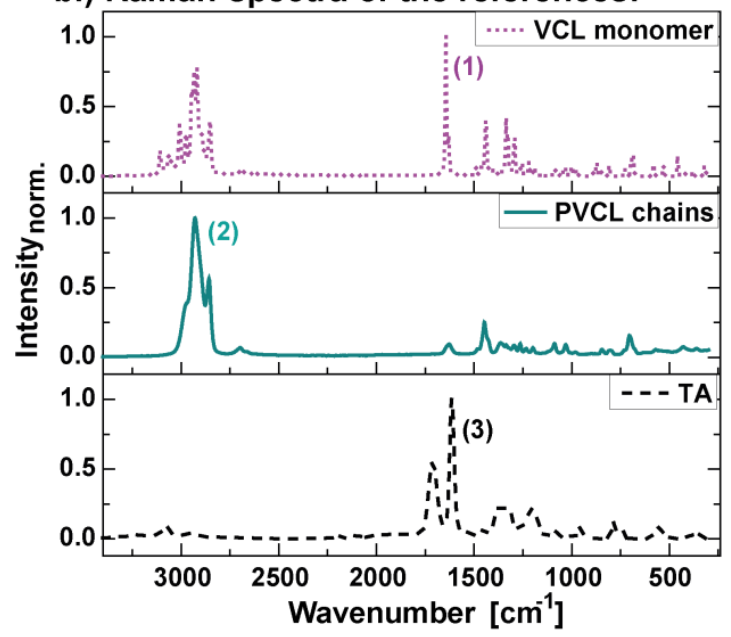

c.) Raman-spectra of the PVCL/TA-microgels:

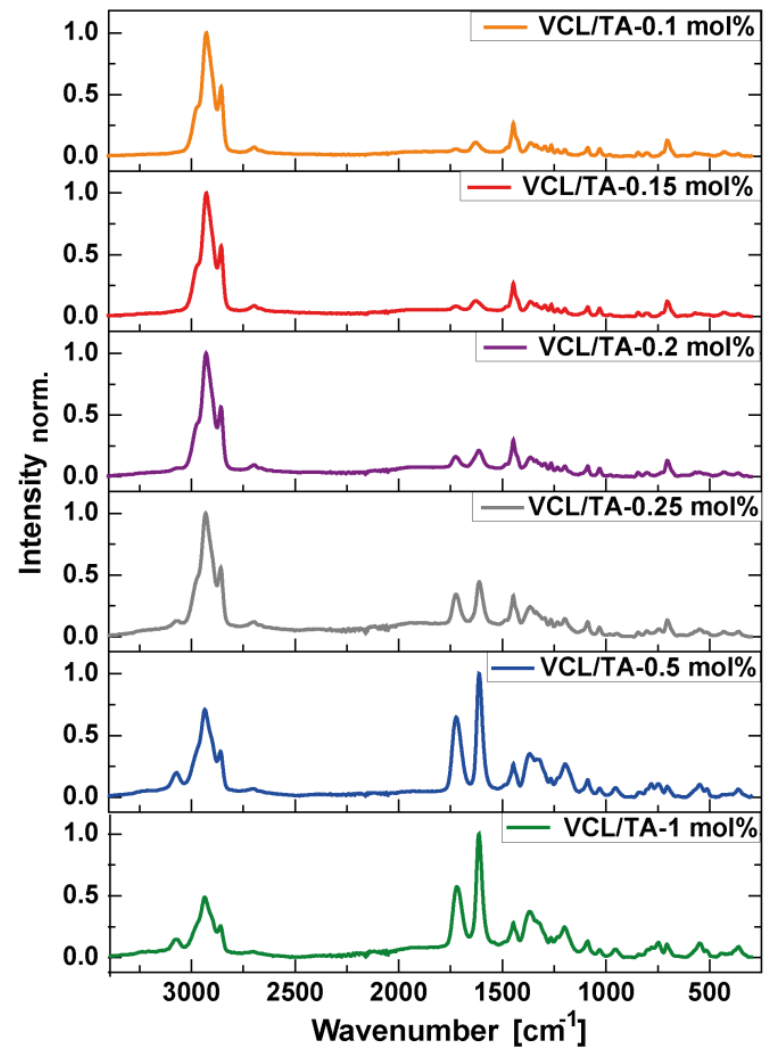

Figure 11. Study of the chemical composition of the PVCL/TA-microgels by Raman spectroscopy.

The chemical composition of PVCL/TA microgels was characterized by Raman spectroscopy (Figure 11). The molecular structures of the references (monomer, PVCL and TA) and their most representative Raman signals are shown in Figure 11a and b. In the spectrum of VCL, the corresponding signal of the vinyl group (1) can be observed at $1643 \mathrm{~cm}^{-1}$. In the case of the PVCL chains, the main signal at $2928 \mathrm{~cm}^{-1}$ correlates with its $\mathrm{C}-\mathrm{H}$ vibrations (2). The signals between 1600 and $1750 \mathrm{~cm}^{-1}$ correspond to the aromatic $\mathrm{C}-\mathrm{C}$ and $\mathrm{C}=\mathrm{C}$-vibrations of the TA (3). The measured curves of the various 
PVCL/TA-microgels are depicted in Figure 11c. In all microgel spectra, the expected characteristic signals were identified. This demonstrates that the microgels are composed exclusively of polymer chains and that the monomer molecules were consumed in the polymerization process. Moreover, an increase of the intensity of the representative signal for TA $\left(\sim 1600 \mathrm{~cm}^{-1}\right)$ can be observed, while the intensity of the PVCL-signal $\left(2928 \mathrm{~cm}^{-1}\right)$ decreases.

The Raman data confirm qualitatively the successful incorporation of the physical crosslinker and variation of its content within the microgels. However, the intensity of the TA peaks in the microgel spectra is unexpectedly high. The real TA-content within the microgels was quantified and the results are summarized in Table 2. To minimize errors and ensure a quantitative determination of the microgels chemical composition, PVCL-TA mixtures of various compositions were prepared to create the calibration curve (supplementary results, Figure 16).

In the first place it can be confirmed positively that the increase of added TA-amount leads to higher TA-contents. However, one observes additionally, that the VCL/TA-ratios do not correspond accurately to the theoretical values and the real TA-amount in microgels is much higher as expected. The reason for this effect could be partial degradation of the VCL under acidic conditions. Imaz et. al. studied previously the effect of the $\mathrm{pH}$ during the polymerization of VCL and these results can be related to postulated hypothesis of degradation. ${ }^{109}$ Furthermore, incomplete monomer conversion and the removal of some non-crosslinked PVCL chains during dialysis are other relevant factors that influence the chemical composition. Nevertheless, the obtained results confirm the successful synthesis of PVCL microgels with variable contents of TA.

Table 2. Theoretical and experimentally determined TA amounts in microgels.

\begin{tabular}{|c|c|c|}
\hline Microgel & $\begin{array}{l}\text { TA theo- } \\
\text { [mol\%] }\end{array}$ & $\begin{array}{c}\mathrm{TA}_{\text {real }} \\
\text { [mol\%] }\end{array}$ \\
\hline VCL/TA-0.1mol\% & 0.1 & 0.24 \\
\hline VCL/TA-0.2mol\% & 0.2 & 1.01 \\
\hline VCL/TA-0.25mol\% & 0.25 & 2.82 \\
\hline VCL/TA-0.5mol\% & 0.5 & 8.96 \\
\hline VCL/TA-1mol\% & 1 & 11.11 \\
\hline
\end{tabular}


Tannic acid is also well-known as a natural antioxidant and an efficient radical scavenger.66, 75, 90, 110 Due to this fact, its presence during the precipitation polymerization of VCL could affect the polymer formation and the microgel composition. The study of the molecular weight of the PVCL- chains within the microgels, as well as its comparison to PVCL synthesized under the same conditions but without crosslinker, is relevant to comprehend the effect of the polyphenol during the radical reaction and its influence on the chemical composition. In view of this, some preliminary measurements via gel permeation chromatography (GPC, supplementary results, Figure 17) were carried out. A direct determination of the molecular weight of the PVCL/TA-microgels by GPC is not possible because of the crosslinked structure. Therefore, measurements of the sample PVCL/TA-0.5mol\% were performed after the disassociation under basic conditions ( $\mathrm{pH} 11)$. The results reveal a surprisingly low number average molecular weight $\left(\mathrm{M}_{\mathrm{n}}\right)$ of $970 \mathrm{~g} \cdot \mathrm{mol}^{-1}$ (polydispersity index (PDI): 1.01). On the other hand, PVCL chains polymerized under the same conditions but without crosslinker showed a molecular weight of $\mathrm{M}_{\mathrm{n}}: 483000 \mathrm{~g} \cdot \mathrm{mol}^{-1}$ (PDI: 2.36). This result suggests the radical scavenging ability of tannic acid. However, it is very important to note that the separation of the components (PVCL chains and TA) after the pH-treatment was extremely difficult and still has to be optimized. It is assumed that a large fraction of polymer chains were still interacting with the TA fraction and remained on the filter. This may explain why only low molecular weight fractions passed the filtration process and were injected into the GPC device. The sample preparation for GPC implies further dilution in dimethylformamide (DMF) and filtration of the product. In the DMF solution, turbidity of the sample and the formation of aggregates could be identified. Due to these facts, the concentration of the PVCL chains was very low and the detection may not represent the real molecular weight of the polymer fraction. A deeper and systematic investigation is hereby necessary. In the following chapter, the effect of TA during the microgel synthesis is discussed.

Using DLS and STEM, the size and morphology of different the PVCL/TA microgels was investigated. Herein, it is important to mention that VCL cannot self-crosslink and therefore, additional crosslinkers are required to obtain stable microgels. ${ }^{33}$ In the present system, the existence of microgels can be justified only by the formation of supramolecular crosslinks between TA and PVCL chains. The influence of the TA amount 
on the microgel structure can be observed clearly by the STEM analysis (Figure 12). Spherical colloids were obtained with TA fractions in a range of 0.1 to $0.2 \mathrm{~mol} \%$ (Figure 12a). However, these microgels are arranged in clusters, especially in the case of the VCL/TA-0.1mol\% sample. One observes that the microgels are agglomerated and not completely isolated. Also, the SEM-images for PVCL/TA samples with $0.1,0.15$ and $0.2 \mathrm{~mol} \%$ TA indicate a broad size distribution. In contrary, the increase of the TAcontent from $0.25 \mathrm{~mol} \%$ up to $1 \mathrm{~mol} \%$ leads to the synthesis of well-defined, rigid and highly monodisperse spherical microgels.

Microgel morphology in dried state:

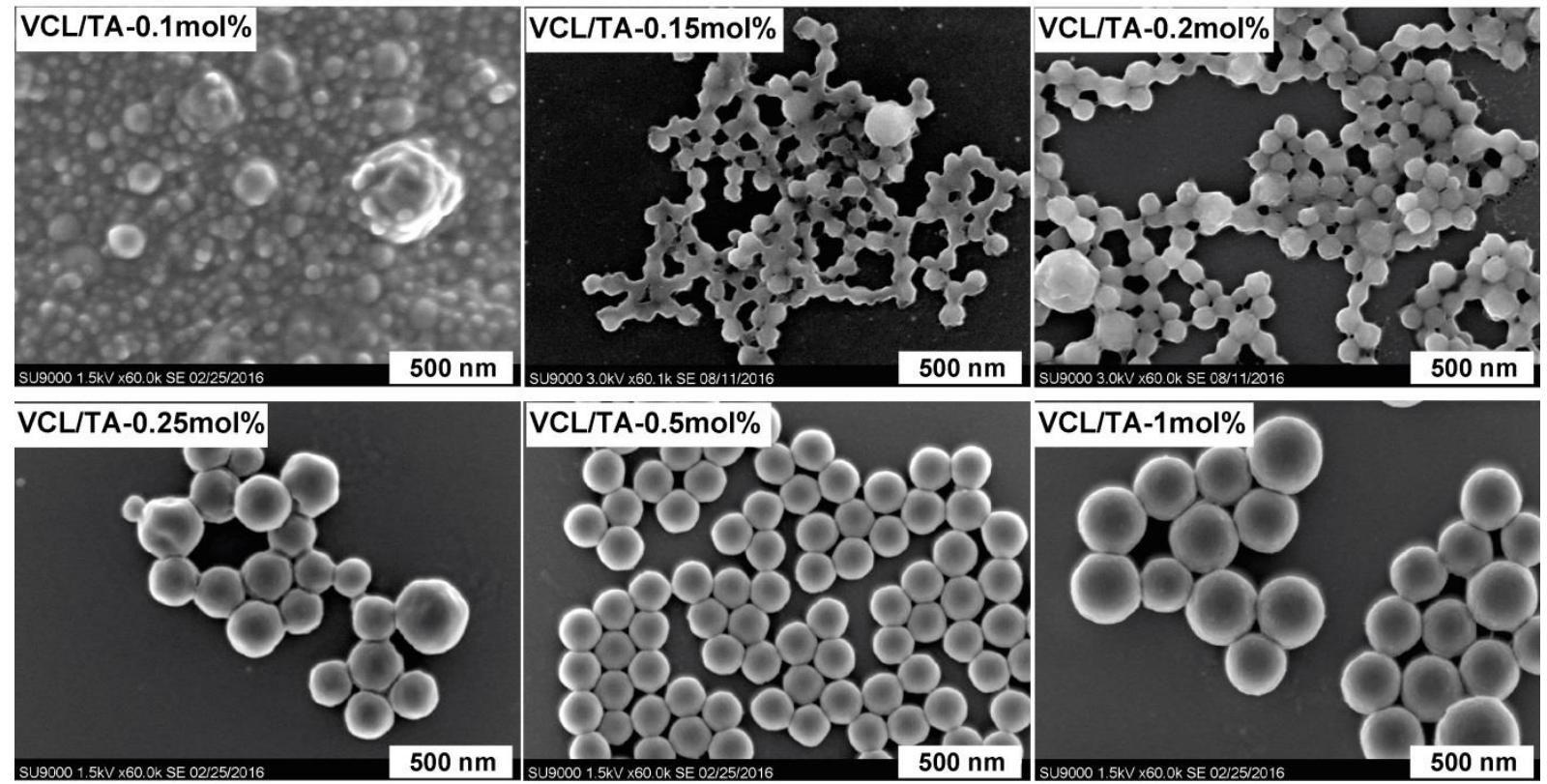

Figure 12. SEM images of PVCL/TA-microgels with various TA-amounts.

The hydrodynamic radii $\left(R_{h}\right)$ and the PDI of the different microgel samples were measured by DLS at room temperature (Figure 13a). It can be concluded, that the higher the TA content within the microgels, the smaller hydrodynamic radius becomes. An increase of the crosslinker amount leads to a higher crosslinking density and thus, to smaller microgel dimensions. This tendency can be observed for the microgels with TA contents up to $0.5 \mathrm{~mol} \%$. Above a TA-fraction of $0.5 \mathrm{~mol} \%$, the $\mathrm{R}_{\mathrm{h}}$ and the PDI increase once again. In this case, the crosslinking density increases with higher crosslinker amounts, although there are more functional groups available. These additional functionalities interact with further polymer chains. Thus, the polymer amount within 
the particle increases and the particle size enlarges. However, the SEM-analysis shows a different trend.

a.) Microgel size and polydispersity:

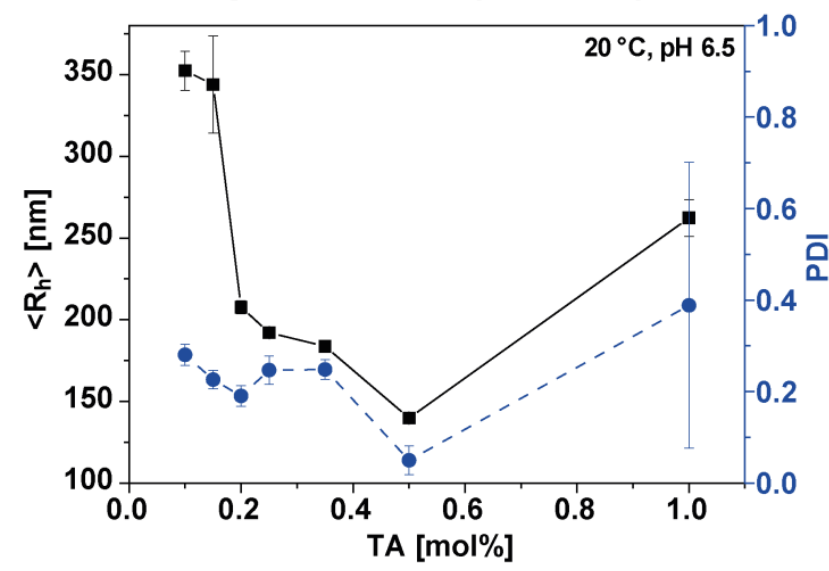

b.) Electrophoretic mobility:

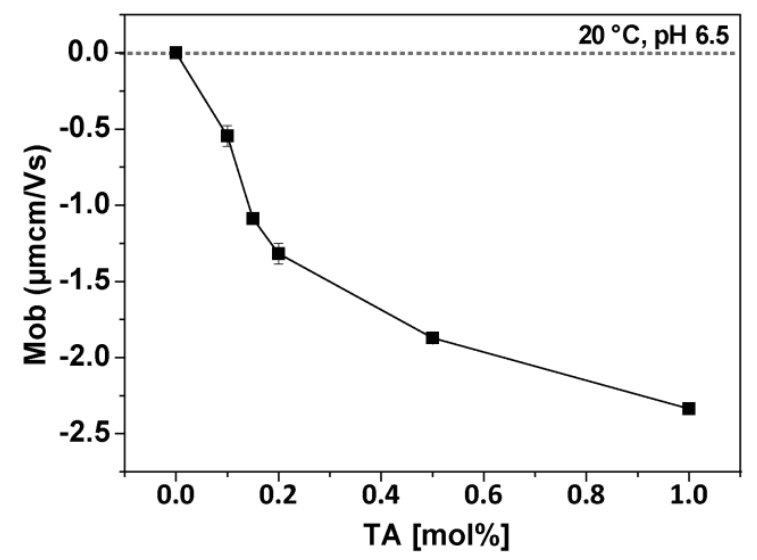

Figure 13. Particle size, PDI values and b.) electrophoretic mobility at $20^{\circ} \mathrm{C}$ and $\mathrm{pH} 6.5$ in dependence of the TA-content.

Contrary to the DLS measurements, higher TA-amounts appear to increase the particle size. This unexpected contradiction can be easily clarified by the drying process and thermoresponsiveness of the microgels. For the SEM characterization, the microgels are in a dried state so that the water is removed from the polymer network. Opposite to this, the DLS measurements are carried out in solution. In this case, the microgels are swollen at room temperature $\left(20^{\circ} \mathrm{C}\right)$. The SEM pictures can be compared to the microgel behavior above their VPTT. A representation of the typical thermal response of the PVCL-based microgels is illustrated in Figure 14.

Thermoresponsiveness of the PVCL/TA-microgels:
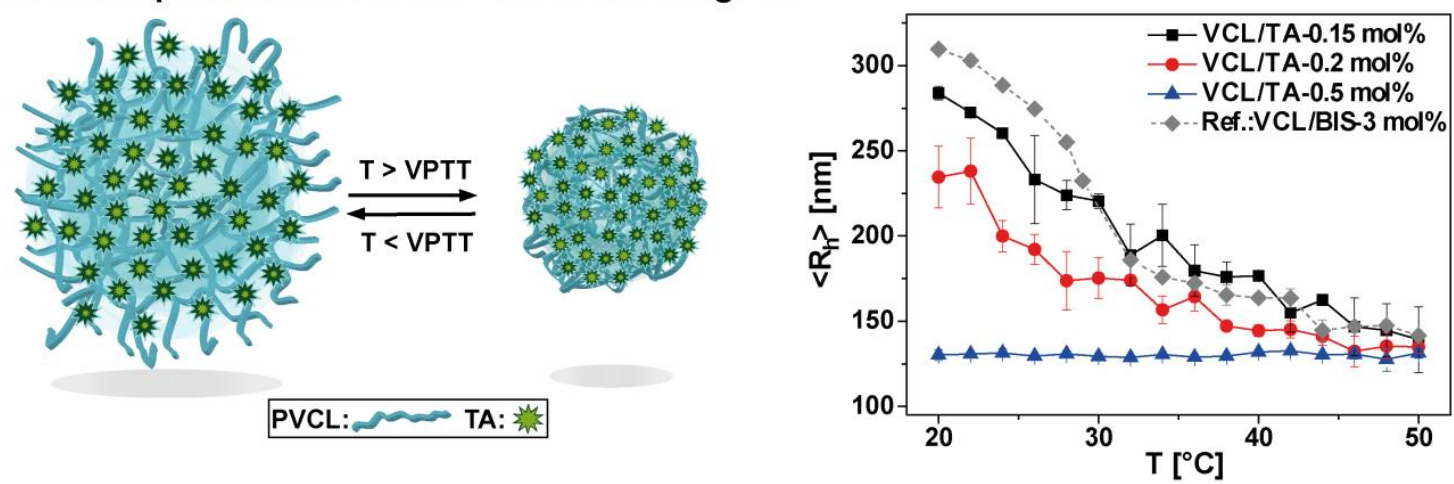

Figure 14. Schematic representation of the thermoresponsive behavior of PVCL-based microgels and hydrodynamic radii of the various PVCL/TA microgels in dependency of temperature measured by DLS. 
The obtained microgels are sensitive to temperature changes and show a VPTT around $32{ }^{\circ} \mathrm{C}$. Bellow the VPTT, the interactions between the polymer chains and water molecules predominate, and the water content as well as the particle volume increase. The inter- and intramolecular interactions between the polymer chains become stronger by increasing the temperature and the water molecules are forced to leave the network. Above the VPTT, the microgels get transformed into the collapsed state.

The thermoresponsiveness of 3 different PVCL/TA microgels as well as a reference (PVCL/BIS-microgels) synthesized with standard crosslinker $N, N^{\prime}$-methylenebisacrylamide (BIS) was characterized by DLS . The reference VCL/BIS-3mol\% microgel shows the well-known thermal properties for PVCL microgels. In the case of the physically crosslinked microgels (PVCL/TA), the results confirm that the TA incorporation has an influence on the hydrodynamic radius and the thermoresponsiveness. The microgels with low TA amounts ( 0.15 and $0.2 \mathrm{~mol} \%$ ) show a thermoresponsive behavior. However, this property diminishes with increased TA-content within the microgels. For the system containing $0.5 \mathrm{~mol} \%$ of TA, there is no thermal sensitivity to be observed. Above the VPTT $\left(50^{\circ} \mathrm{C}\right)$, all microgels show similar hydrodynamic radii $(\sim 140 \mathrm{~nm})$. These results correlate with the microgel dimensions obtained by SEM. The microgels with a TA content of $0.5 \mathrm{~mol} \%$ represents rigid spheres, and this correlates with the absence of thermal responsiveness. This effect would also explain the size trend observed by the microscopy. These measurements were carried out in the collapsed state. Microgels with a TA contents below $0.2 \mathrm{~mol} \%$ are softer. There are more interactions between the polymer chains and the water molecules in the swollen state, and thus, these microgels are more hydrophilic and soft. Due to this fact, the gels shrink strongly during the drying process and achieve smaller diameters then microgels with higher TA content, in a complete free-solvent state. This primary study confirms certainly not only the successful synthesis of the supramolecular PVCL microgels using tannic acid as a physical crosslinker, but also that their thermal behavior can be tuned by the crosslinker content.

The surface charge of the synthesized microgels was characterized by measurements of the electrophoretic mobility (Mob) at $20{ }^{\circ} \mathrm{C}$ and neutral $\mathrm{pH}(\sim 6.5)$. The obtained results represent the expected trend (Figure 13b). The higher the percentage of TA within the 
microgels, the more negatively charged the particle surface is. The negative charge of the microgels is given by the hydroxyl groups of the polyphenol. These results demonstrate that the size and the thermo-responsiveness of the microgels can be controlled, while the electrical surface properties can be varied in a facile manner. Thus, the physically crosslinked PVCL/TA microgels can be very attractive for different applications, not only because of the biocompatibility of the building blocks, but also owing to their responsiveness to environmental changes.

pH-stability of the PVCL/TA-microgels:
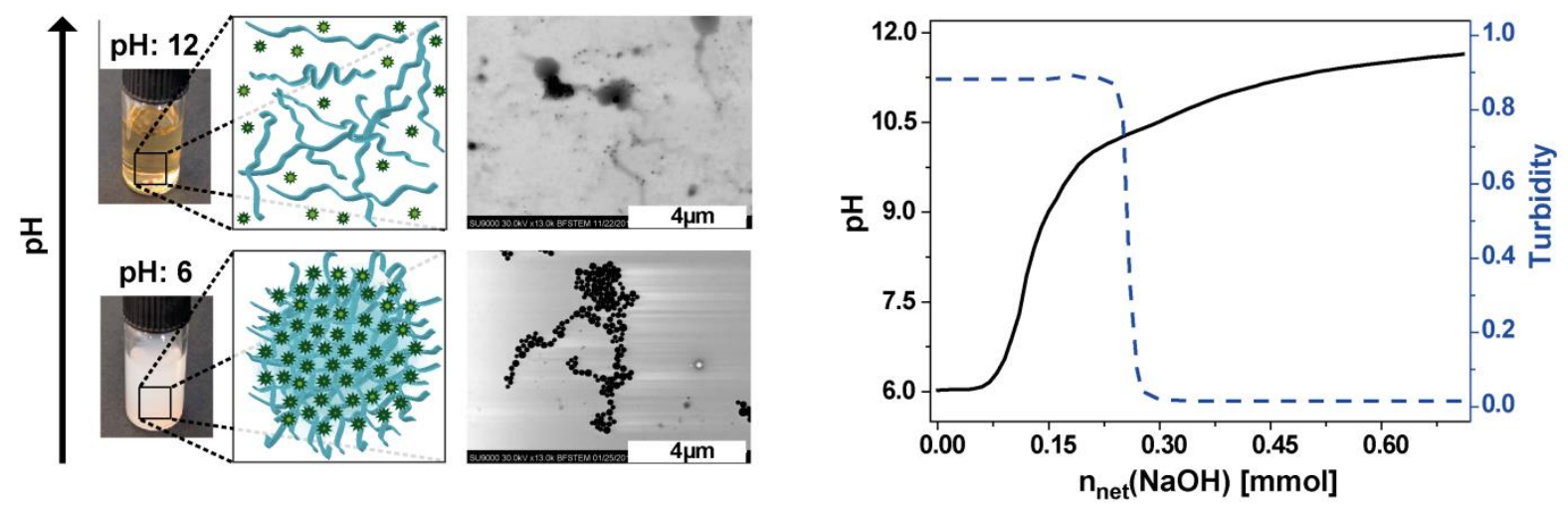

Figure 15. Illustration of the $\mathrm{pH}$ sensitivity, SEM-images of the microgels at different pH values, as well as titration and turbidity curves measured by adding $\mathrm{NaOH}(0.1 \mathrm{M})\left(\mathrm{VCL} / \mathrm{TA} 0.5\right.$ mol $\left._{\text {theo. }} \%\right)$ at room temperature.

The pH-sensitivity of TA-assembled materials has been studied strongly within the last years. ${ }^{74,84,96,102,111-112}$ In an exploratory manner, we investigated the pH-triggered disintegration of the PVCL/TA-microgels (0.5 mol\%). The $\mathrm{pH}$ of the aqueous solution was increased from 6 to 12 by adding $\mathrm{NaOH}$ and the $\mathrm{pH}$ and turbidity changes were monitored (Figure 15). As described previously, TA serves as an H-donor and thus, it interacts with the PVCL chains by H-bonding during the microgel formation. Due to its high $\mathrm{pK}_{\mathrm{a}}$ value $(\sim 8.5),{ }^{74}$ the microgels can be synthesized in aqueous media and remain stable under neutral conditions. By increasing the $\mathrm{pH}$ to 10 , the phenolic groups of TA start to deprotonated. At a pH of 11, the deprotonation process is completed and the microgels degrade. The turbidity curve correlates with the solution pictures and TEMimages. One can observe clearly that the color of the solution changes from turbid white ( $\mathrm{pH}$ 6) to clear yellow ( $\mathrm{pH} 12$ ). The yellowish coloration suggests the release of TA molecules. The microscopy pictures presented in Figure 15 confirmed additionally the desintegration of the microgels. The small polymer clusters visible after the degradation process could be originated from some additional crosslinks introduced by the 
interaction of phenolic groups with PVCL oligoradicals. As demonstrated recently by Xue et al., the presence of cathecol-containing monomers during the precipitation polymerization of NIPAAm may lead to crosslinking reactions. ${ }^{113}$ However, detailed studies of the degradation process and structure of disassembly products of PVCL/TA microgels should be performed in the future to get better understanding of internal microgel structure. We believe that $\mathrm{pH}$-triggered disintegration of PCVL/TA microgels demonstrates not only their supramolecular nature, but also highlights strongly the importance of this development. To our knowledge, there are only few examples of physical crosslinked microgels in the literature, and all of them imply complex procedures and the synthesis of complicated molecular building blocks. ${ }^{51,53-56}$ Based on these results, the novel supramolecular microgels are promising candidates for numerous biomedical applications including controlled drug delivery and molecular engineering.

\subsection{Conclusions}

In this section of the thesis, a facile and simple synthesis of supramolecular microgels was established based on PVCL physically crosslinked by tannic acid. Due to its ability to form hydrogen bonds, confirmed by preliminary studies, TA served as a unique physical crosslinker for the formation of stable PVCL-based microgels. The variation of the crosslinker content within the microgels enabled the control of the microgel properties. The increase of the TA amount led to an increase of the microgel density meaning the production of harder and smaller particles. This effect influenced the water uptake ability of the microgels leading to a repression of the thermo-responsiveness. Moreover, it was proved that polymer chains are necessary to support the microgel formation. The electrical surface charge of the supramolecular microgels could be varied successfully in dependency of the TA content. By Raman spectroscopy, the successful incorporation of TA into the microgel network was confirmed and quantified. Finally, the the pHsensitivity of the PVCL/TA microgels was analyzed and their disassembly under basic $\mathrm{pH}$ in aqueous solution was proved. This primary study proposes not only an innovative method for the production of responsive nanomaterials, but also combines the features of biological molecules and stimuli-responsive polymer chains by polymerizationinduced self-assembly. The novel PVCL/TA microgels could therefore be interesting for 
applications in the biomedical field, where stimuli-responsiveness and a controlled degradation processes play an essential role. 


\subsection{Supplementary Results}

\section{Quantification of the TA-content by Raman spectroscopy}

Table 3. Calculated values for the calibration curve.

\begin{tabular}{|c|c|c|c|c|}
\hline $\mathbf{n}_{\text {TA }}[\%]$ & $\begin{array}{c}\mathbf{n}_{\text {TA }} / \mathbf{n}_{\text {PVCL }} \\
{[\% / \%]}\end{array}$ & $\mathbf{I}_{\text {TA }}$ & I PVCL & $\mathbf{I}_{\text {TA }} / \mathbf{I}_{\text {PVCL }}$ \\
\hline 0.2 & 0.002 & 0.004 & 0.352 & 0.011 \\
\hline 0.5 & 0.005 & 0.013 & 0.348 & 0.038 \\
\hline 1 & 0.010 & 0.032 & 0.350 & 0.090 \\
\hline 2.5 & 0.026 & 0.075 & 0.343 & 0.220 \\
\hline 5 & 0.053 & 0.113 & 0.255 & 0.443 \\
\hline 10 & 0.111 & 0.111 & 0.124 & 0.896 \\
\hline 25 & 0.333 & 0.119 & 0.036 & 3.333 \\
\hline 50 & 1.000 & 0.109 & 0.012 & 9.083 \\
\hline
\end{tabular}

\section{a.) Raman spectra of calibration}

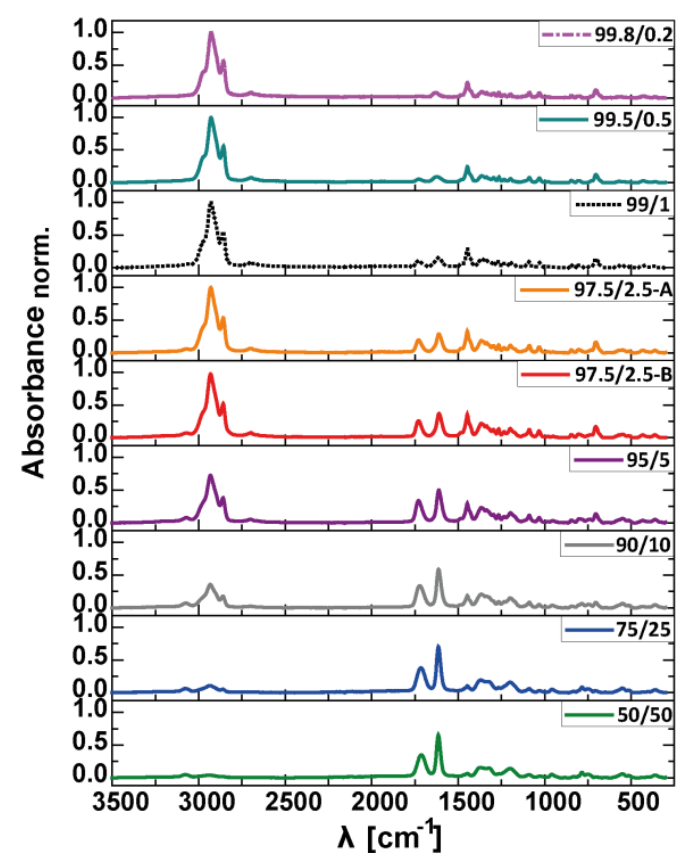

b.) calibration graph

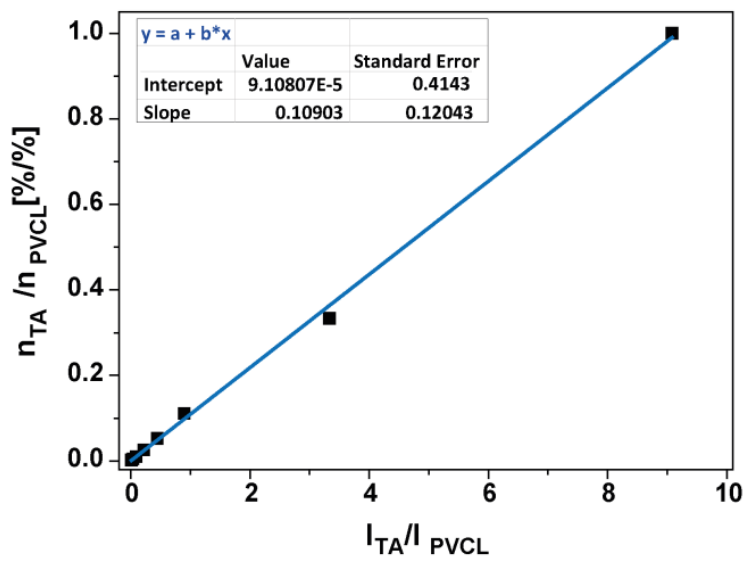

Figure 16. Raman calibration for the quantification of the real TA-content. 
Table 4. Quantification of the real TA-content within the PVCL/TA-microgels.

\begin{tabular}{|c|c|c|c|c|c|}
\hline $\mathbf{n}_{\text {TA,theo }}[\%]$ & $\mathbf{I}_{\text {TA }}$ & $\mathbf{I}_{\mathbf{P V C L}}$ & $\mathbf{I}_{\mathrm{TA}} / \mathbf{I}_{\mathbf{P V C L}}$ & $\begin{array}{c}\mathbf{n}_{\text {TA }} / \mathbf{n}_{\mathbf{P V C L}} \\
{[\% / \%]}\end{array}$ & $\mathbf{n}_{\text {TA,real }}[\mathbf{\%}]$ \\
\hline 0.10 & 0.005 & 0.238 & 0.021 & 0.002 & 0.24 \\
\hline 0.15 & 0.006 & 0.205 & 0.030 & 0.003 & 0.34 \\
\hline 0.20 & 0.019 & 0.203 & 0.092 & 0.010 & 1.01 \\
\hline 0.25 & 0.038 & 0.143 & 0.264 & 0.029 & 2.82 \\
\hline 0.50 & 0.099 & 0.110 & 0.900 & 0.098 & 8.96 \\
\hline 1.00 & 0.079 & 0.069 & 1.142 & 0.125 & 11.11 \\
\hline
\end{tabular}

\section{Preliminary analysis of the molecular weight:}

a.) VCL/TA- $0.5 \mathrm{~mol} \%$ after $\mathrm{pH}$-degradation

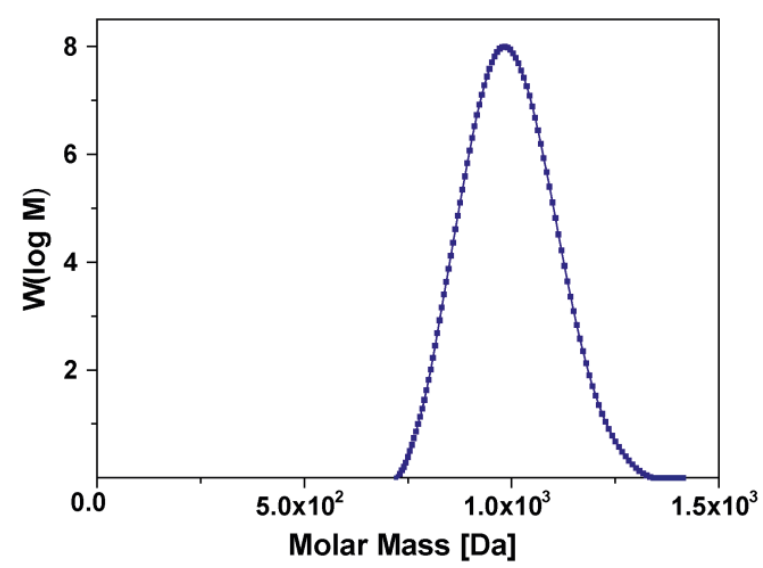

b.) PVCL chains by

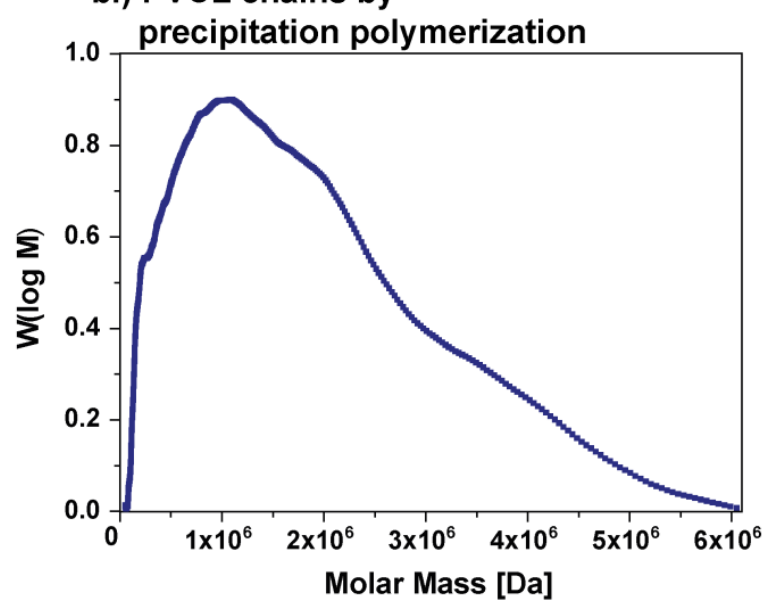

Figure 17. Initial study of the molecular weight via GPC (in DMF). Molecular mass distribution of a.) PVCL chains contained in the microgels (VCL/TA-0.5mol\% sample after the pH degradation) and b.) PVCL chains synthesized by precipitation polymerization without crosslinker. 


\subsection{Experimental Section}

\subsubsection{Materials}

2,2'-Azobis-(2-methylpropionamidine) dihydrochloride (AMPA, 97\%, Aldrich), cetyltrimethylammonium bromide (CTAB, $\geq 97.0 \%$, Merck Millipore), tannic acid (TA, Sigma-Aldrich), sodium hydroxide solution (0.1 N, Sigma-Aldrich), $\mathrm{FeCl}_{3} \cdot 6 \mathrm{H}_{2} \mathrm{O}$ (reagent grade, 97 \%, Sigma-Aldrich), and dialysis membranes (Zellu Trans/Roth MWCO 3500 and 12000-14000, Carl Roth) were used without further treatment. $N$-vinylcaprolactam (VCL, $98 \%$, Aldrich) was distillated under vacuum before use. Linear PVCL was synthesized via RAFT polymerization by Chaolei Hu (Research group of Prof. A. Pich, DWI-Leibniz-Institute for Interactive Materials). Deionized water was used for all experiments.

\subsubsection{Analysis of TA Interactions as Proof of Concept}

\section{a) Interactions between PVCL and TA}

The formation of stable interactions between PVCL and TA was characterized by a visual analysis. Linear PVCL $\left(\mathrm{M}_{\mathrm{n}} \sim 30 \mathrm{~kg} \cdot \mathrm{mol}^{-1}\right)$ was dissolved in $\mathrm{H}_{2} \mathrm{O}$ at room temperature to obtain an aqueous solution (10 $\left.\mathrm{mg} \cdot \mathrm{mL}^{-1}\right)$. Then, the solution ( $3 \mathrm{~mL}$ ) was heated up to $50{ }^{\circ} \mathrm{C}$. After reaching the desired temperature, a certain amount of an aqueous TAsolution (30 $\mu \mathrm{L}, 1 \mathrm{mM})$ was added to one of the PVCL sample. Finally, the solution was cooled down to room temperature.

\section{b) Isothermal Titration Calorimetry}

The thermodynamic profile of the interactions between PVCL and TA was characterized by ITC on a TAM III calorimeter from the company TA Instruments. An aqueous solution of linear PVCL-chains $\left(300 \mu \mathrm{L}, 10 \mathrm{mmol} \cdot \mathrm{L}^{-1}\right)$ was titrated with an aqueous solution of TA $\left(250 \mu \mathrm{L}, 1 \mathrm{mmol} \cdot \mathrm{L}^{-1}\right)$ at $25{ }^{\circ} \mathrm{C}$. As reference a pure linear PVCL aqueous solution was used $\left(425 \mu \mathrm{L}, 10 \mathrm{mmol} \cdot \mathrm{L}^{-1}\right)$. The concentration of the PVCL chains was calculated from the monomer. The titrant TA was added to the analysis cell over 50 injections (5 $\mu \mathrm{L}$ steps within $10 \mathrm{~s}$ each). The equilibration time between the injections was a period of 15 min. The raw data was analyzed by the software NanoAnalyze (TA Instruments). 


\section{c) Formation of microgel/metal ion-based hydrogels}

Microgel/metal ion-based hydrogels were produced based on a combination of various building blocks in different stages An aqueous solution of TA was prepared $(100 \mu \mathrm{L}$, $\left.50 \mathrm{mg} \cdot \mathrm{mL}^{-1}\right)$ and PVCL dissolved in $\mathrm{H}_{2} \mathrm{O}\left(350 \mu \mathrm{L}, 70 \mathrm{mg} \cdot \mathrm{mL}^{-1}\right)$ as well as PVCL-based microgels dispersed in $\mathrm{H}_{2} \mathrm{O}\left(50 \mu \mathrm{L}, 50 \mathrm{mg} \cdot \mathrm{mL}^{-1}\right)$ were added to the solution. The mixture was shaken for $20 \mathrm{~min}$ at RT. Subsequently, an aqueous solution of $\mathrm{FeCl}_{3}(25 \mu \mathrm{L}$, $32 \mathrm{mg} \cdot \mathrm{mL}^{-1}$ ) was added to the TA/polymer mixture. Finally, the $\mathrm{pH}$-value of the solution was decreased by addition of $\mathrm{NaOH}(50 \mu \mathrm{L}, 1 \mathrm{M})$ and the sample was shaken further overnight.

\subsubsection{Microgel Synthesis}

\section{a) Route I: Synthesis of PVCL/TA-Microgels by Batch Precipitation Polymerization}

PVCL/TA-microgels were synthesized via precipitation polymerization. The polymerization was performed in a double wall glass reactor. VCL ( $\left.2 \mathrm{~g}, 139.2 \mathrm{~g} \cdot \mathrm{mol}^{-1}\right)$ was dissolved in deionized water $(150 \mathrm{~mL})$ at room temperature, CTAB $(0.006 \mathrm{~g}$, $364.45 \mathrm{~g} \cdot \mathrm{mol}^{-1}$ ) was added, and to the monomer solution was stirred 30 min under $\mathrm{N}_{2}$ atmosphere. Subsequently, the crosslinker TA (0.0245 - 0.2469 g, $1701.2 \mathrm{~g} \cdot \mathrm{mol}^{-1}$ ) (Table 5) was added to the reaction mixture, and the reactor was heated up to $70{ }^{\circ} \mathrm{C}$. The polymerization was initiated by the addition of AMPA $\left(0.05 \mathrm{~g}, 271.19 \mathrm{~g} \cdot \mathrm{mol}^{-1}\right)$ and performed for 5 hours. After cooling down, the PVCL/TA-microgels were purified by dialysis in deionized water for 4 days, and lyophilized.

Table 5. Quantities of TA used for the microgel synthesis, hydrodynamic radii and PDI of the PVCL/TAmicrogels measured via DLS.

\begin{tabular}{|c|c|c|c|}
\hline Microgel & TA[g] & $<_{\mathrm{h}-20^{\circ} \mathrm{C}}>[\mathbf{n m}]$ & PDI $_{20^{\circ} \mathrm{C}}$ \\
\hline VCL/TA-0.1mol\% & 0.0245 & $352.4 \pm 12,0$ & $0.280 \pm 0.023$ \\
\hline VCL/TA-0.15mol\% & 0.0367 & $343.9 \pm 29.6$ & $0.226 \pm 0.020$ \\
\hline VCL/TA-0.20mol\% & 0.0490 & $207.7 \pm 4.0$ & $0.191 \pm 0.022$ \\
\hline VCL/TA-0.25mol\% & 0.0613 & $192.1 \pm 2.0$ & $0.247 \pm 0.031$ \\
\hline VCL/TA-0.5mol\% & 0.1228 & $139.8 \pm 2.1$ & $0.050 \pm 0.032$ \\
\hline VCL/TA-1mol\% & 0.2469 & $262.3 \pm 11.2$ & $0.389 \pm 0.312$ \\
\hline
\end{tabular}


In view of the temperature and surfactant influence, 2 further experiments were performed based on VCL/TA-0.5mol\% recipe.

Influence of the temperature: During the microgel synthesis, the thermal equilibration time was modified. Therefore, the AMPA was added to the monomer-TA solution at $70{ }^{\circ} \mathrm{C}$ after an equilibrating period of $1 \mathrm{~h}$.

Influence of surfactant: The effect of the utilization of a cationic surfactant during the synthesis of the PVCL/TA-microgels was analysed. Herein, and additional sample was prepared without CTAB.

\subsubsection{Characterization Methods}

\section{a) In-situ Dynamic Light Scattering Measurements}

The particle growth was monitored in-line during the microgel-synthesis (Route I) by DLS using Nano-Flex DLS (Nanotrac Wave Optics, Microtrac; serial number: SN W3227) equipped with a $5 \mathrm{~mW}$ solid-state diode laser $(780 \mathrm{~nm})$.

\section{b) Dynamic Light Scattering Measurements}

The microgel size/size distribution was characterized by DLS using ALV/CGS-3 Compact Goniometer System from the company ALV-GmbH with an ALV/LSE 5004 tau digital correlator and a JDS uniphase laser operating at $632.8 \mathrm{~nm}$. The scattering angle was fixed $\left(\theta=90^{\circ}\right)$ for all measurements. The temperature dependent experiments were performed in a range from 20 to $60^{\circ} \mathrm{C}$. All samples were filtered before the measurements with Chromafil@ Xtra syringe filters $(0.45$ or $1.20 \mu \mathrm{m})$. The results were obtained by ALV-Correlator Software V3.0.

\section{c) Electrophoretic Mobility Measurements}

The surface charge of the PVCL/TA-microgels was determined by measurements of the electrophoretic mobility (Mob) with a Zetasizer NanoZS (Malvern). All microgels were measured at $20^{\circ} \mathrm{C}$ and neutral pH ( 6.5) after dispersing them in HPLC-grade water.

\section{d) Scanning Transmission Electron Microscopy}

The morphology of the microgels was studied by SEM and TEM on an Ultra-high Resolution Scanning Electron Microscope SU9000 (Hitachi-High Technologies America, 
Inc.). $100 \mu \mathrm{L}$ of each microgel solution $\left(0.1 \mathrm{~g} \cdot \mathrm{L}^{-1}\right)$ were diluted in $1 \mathrm{~mL}$ deionized water. $20 \mu \mathrm{L}$ of it were dropped on a TEM-grid (Carbon Film 200 Mesh Copper Grids, Electron Microscopy Sciences) and dried at room temperature overnight. The samples were sputtered with $2 \mathrm{~nm}$ carbon before the measurements.

This work was performed by Sabrina Mallmann in part at the Center for Chemical Polymer Technology CPT, which is supported by the EU and the federal state of North Rhine-Westphalia (grant no. EFRE 3000883 02).

\section{e) Raman Spectroscopy Analysis}

The incorporation of the physical crosslinker TA in to the microgel network was confirmed and quantified by Raman spectroscopy on a Bruker RFS 100/S Raman spectrometer with a Nd:YAG laser (1064 nm). The measurements were carried out with a spectral resolution of $14 \mathrm{~cm}^{-1}$, power of $200 \mathrm{~mW}$ and 1000 scans. The results were analyzed using the software OPUS 4.0.

The quantification of the TA content within the microgels was performed using a calibration curve. Therefore, linear PVCL and TA were mixed in specific ratios and homogenized in methanol. The solvent was removed by evaporation at room temperature and the calibration samples were measured by Raman spectroscopy. The obtained results for the calibration and the corresponding graph are displayed in the next section.

\section{f) Potentiometric Titration and Turbidity Measurements}

Aqueous microgel solutions (0.1 wt.-\%) were treated with $\mathrm{NaOH}(0.1 \mathrm{M})$ in $100 \mu \mathrm{L}$ steps until a pH of 12 was achieved. The titration curves were measured on a Metrohm Titrando 905.

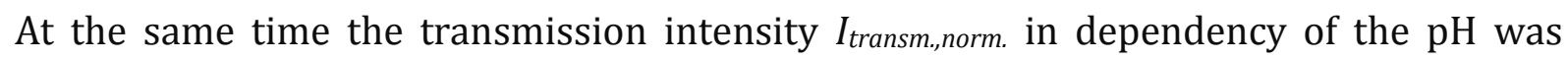
measured using an optrode $(640 \mathrm{~nm})$. The obtained data were normalized and related to the turbidity as follows:

$$
\text { Turbidity }=\frac{1}{I_{\text {transm.,norm. }}}
$$




\section{g) Gel Permeation Chromatography}

Molecular weights and molecular weight distributions were determined by GPC using DMF as eluent. The measurements were performed using an Agilent 1100 system equipped with a dual RI-/Visco detector (ETA-2020, WGE). The eluent contained $1 \mathrm{~g} \cdot \mathrm{L}^{-1}$ $\mathrm{LiBr}$ ( $\geq 99 \%$, Sigma-Aldrich) as internal standard. The sample solvent contained traces of distilled water as internal standard. One pre-column $(8 \times 50 \mathrm{~mm})$ and four PSS GRAM gel columns $(8 \times 300 \mathrm{~mm})$ were applied at a flow rate of $1.0 \mathrm{~mL} \cdot \mathrm{min}^{-1}$ at $40{ }^{\circ} \mathrm{C}$. The diameter of the gel particles measured $10 \mu \mathrm{m}$, the nominal pore widths were 30,100, 100 and $3000 \AA ̊$ A. Calibration was achieved using narrow distributed PS standards. The results were evaluated using the PSS WinGPC UniChrom software (Version 8.1.1). 


\section{Synthesis of Supramolecular PVCL/TA-Microgels by an Optimized Semi-Batch Precipitation Polymerization Approach}

\subsection{Introduction}

Covalent crosslinkers with radical reactive groups are utilized mostly in microgel design. Such components participate actively during the polymerization and become incorporated into the polymer network by chemical interactions. However, the usage of covalent crosslinking and its high stability hinder the design of degradable colloids. Therefore, the implementation of supramolecular chemistry in microgel synthesis would lead to more dynamic and adaptive assemblies.

Due to the well-known ability of polyphenols to complex proteins, these biomacromolecules have been studied intensively in view of supramolecular assembly. Their interactions with natural and synthetic polymers have been thus analyzed strongly. Especially in the case of TA, its property to form stable non-covalent structures with polymeric materials by hydrogen bonding has been reported successfully. $45,74,97$, 112,114 This special feature can enable the incorporation of TA as supramolecular agent in the synthesis of responsive and non-covalent assembled nanomaterials.

\subsection{Results and Discussion}

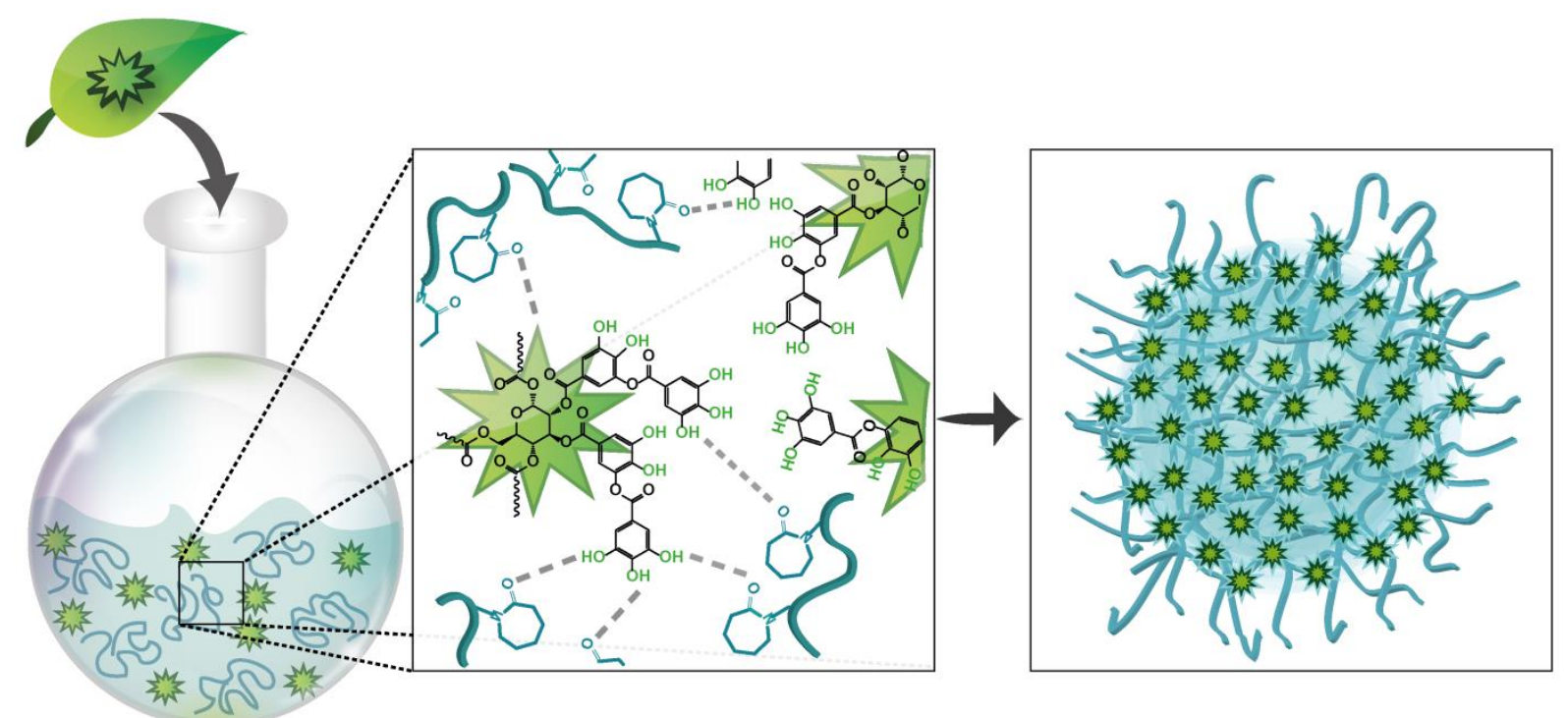

Figure 18. Illustrated microgel formation based on H-bonding between tannic acid and PVCL. 
In the previous chapter, the preliminary synthesis of supramolecular microgels based on the natural polyphenol TA and the responsive polymer PVCL is reported. ${ }^{115}$ The incorporation of tannic acid as non-covalent crosslinker was achieved during the microgel synthesis via precipitation polymerization (Figure 18). Herein, the variation of PVCL-to-TA ratio revealed the control of the material properties such as chemical composition, size and density degree. The physical crosslinking between the polymer chains and the polyphenol was confirmed through a pH-triggered disassembly under basic conditions. In conclusion, this initial work stated an alternative for innovative supramolecular colloidal gels with a broad spectrum of structural, as well as stimuliresponsive and degradable properties. Nonetheless, our preliminary discoveries led to further outstanding questions in terms of understanding the supramolecular aspect of the system and the optimization of its properties.

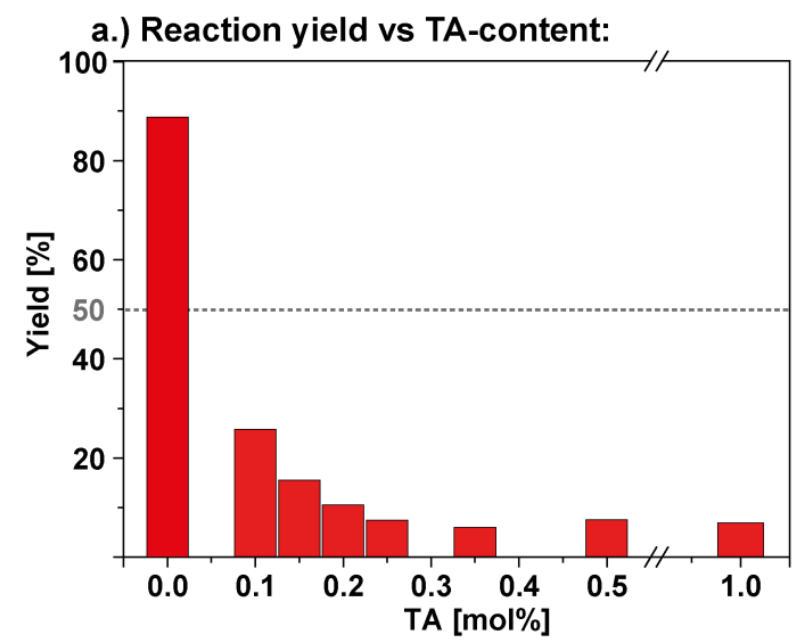

\section{b.) Stability of $\mathrm{VCL}$ at $\mathrm{pH} 4$ and $\mathrm{RT}$ :}

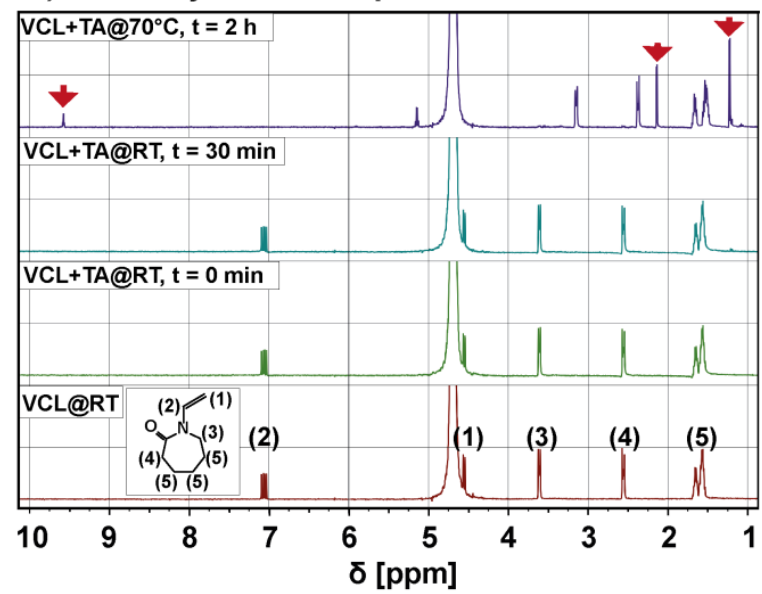

c.) H-NMR of TA at reaction conditions:

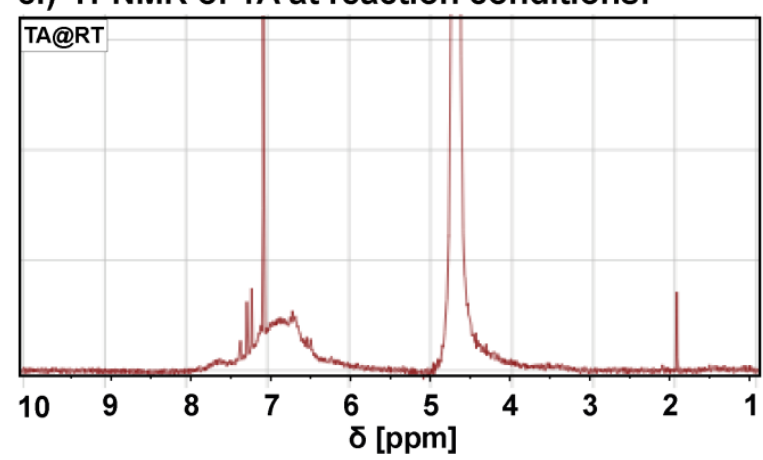

Figure 19. a) Yields of the PVCL/TA-microgels in dependency of the crosslinker content and b) ${ }^{1}$ H-NMR-study of the stability of VCL in the presence of TA at RT. c) ${ }^{1} \mathrm{H}-\mathrm{NMR}$ spectrum of TA.

Based on this, the subsequent studies were carried out in order to determine the microgel yield as well as the monomer conversion in dependency of the time. Due to the fact that the molecular weight and thus, the amount-of-substance of microgels cannot be 
determined accurately until now, the calculation of the yield values was carried out gravimetrically. The determination of the microgel yield allows a qualitative analysis of the conversion, since both reaction terms are proportionally related. It is important to remark that the microgel yield does not correspond exactly to the monomer conversion, since it is possible that not all the polymer chains are integrated within the microgel network. The resulting yields in dependency of the TA molar ratio are illustrated in Figure 19a. VCL was polymerized without any crosslinker (TA $=0.0 \mathrm{~mol} \%$ ) as a reference. Normally, its radical polymerization leads to very high yields and conversion values. In terms of microgel production, yields of up to $90 \%$ can be easily obtained. However, by the production of the PVCL/TA-microgels, the integration of TA as a crosslinker causes a strong decrease of the yield, leading to very low values below $6 \%$. For a better understanding of this limitation, we analyzed the influence of the synthesis conditions.

The initial synthesis of the microgels is based on 3 main steps (Chapter 2, Figure 8). Firstly, the addition of TA to the monomer solution takes place at room temperature (RT) before the polymerization. Subsequently, the VCL units and the crosslinker interact instantly forming hydrophobic structures. As a final step, the reaction temperature is set up to $70{ }^{\circ} \mathrm{C}$, and the polymerization is started by the addition of the initiator. Herein, $\mathrm{pH}$ and temperature are both relevant factor during the microgel formation. In the first phase, the $\mathrm{pH}$ in the solution decreases to a value of 4 after the crosslinker addition. On the other hand, the temperature change also plays an important role before the initiation. The stability of VCL in the presence of TA was thus determined under these conditions.

For the analysis, the monomer was dissolved in distilled water and stirred at RT. Subsequently, TA was added to the solution $(\mathrm{t}=0 \mathrm{~min})$, and the mixture was stirred further for $30 \mathrm{~min}$. The concentrations and VCL-to-TA ratio corresponded to the values used for the synthesis of microgels with $0.5 \mathrm{~mol} \%$ of TA. Different samples were withdrawn and the stability of the monomer structure was monitored by proton nuclear magnetic resonance $\left({ }^{1} \mathrm{H}-\mathrm{NMR}\right)$ (Figure $19 \mathrm{~b}$ ). Due to the low concentration of TA in the solution, its signals are weak and not relevant for this analysis (Figure 19c). The measured spectra show clearly that the signals of the monomer do not change after the 
addition of the TA. Even after $30 \mathrm{~min}$, all detected signals and their intensity remain constant, and there is no influence of the $\mathrm{pH}$ change on the VCL structure. The presence of TA does not affect the monomer stability at RT. However, one observed some modifications after heating up the solution to $70^{\circ} \mathrm{C}$. In order to determine the cause for the low microgel yield, we repeated the experiment at this temperature. The measured ${ }^{1}$ H-NMR-spectra are shown in Figure 20a.

a.) Stability of $\mathrm{VCL}$ at $\mathrm{pH} 4$ and $70^{\circ} \mathrm{C}$ :

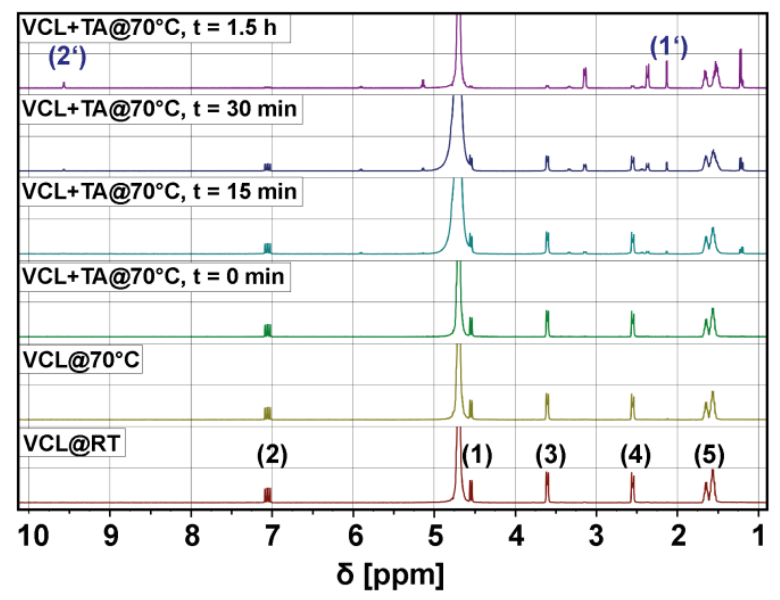

b.) Hydrolysis of VCL at reaction conditions:

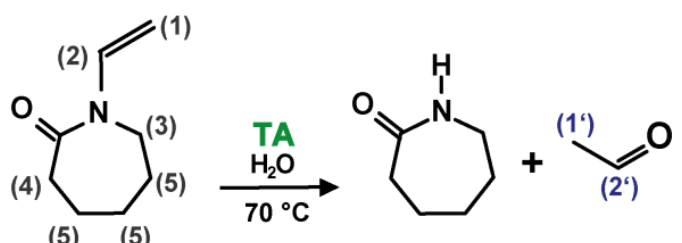

Figure 20. a) ${ }^{1} \mathrm{H}-\mathrm{NMR}$-study of the stability of VCL in the presence of TA and b) schematic representation of the VCL hydrolysis in the presence of TA at the polymerization temperature $\left(70^{\circ} \mathrm{C}\right)$.

By increasing the temperature of the monomer solution, the structure of pure VCL remains stable. Nevertheless, the addition of tannic acid at the polymerization temperature leads to structural changes. The intensity of the signals (1) and (2) starts to decrease after $15 \mathrm{~min}$, and after $1.5 \mathrm{~h}$, these peaks disappear. Both signals correspond to the vinyl group of VCL, which is responsible for the radical polymerization. Their intensity reduction reveals a relevant cause for the low yields. Without the vinyl functionality of the monomer, the initiation and the propagation cannot take place. Furthermore, new signals can be noticed at 2.4 and $3.2 \mathrm{ppm}(\mathrm{t}=15 \mathrm{~min})$. Contrary to the monomer signals, their intensity increases with time. Both unknown peaks can be related to a shifting of the signals (3) and (4) caused by the changed structure of the monomer or by interactions with byproducts. These results can be compared to the previous studies of Imaz et. al.109 This research group studied the $\mathrm{pH}$-influence on the emulsion polymerization of VCL, and reported the hydrolysis of the monomer under acidic conditions as a competitive reaction for the propagation. The hydrolysis mechanism proposed leads to caprolactam and acetaldehyde as products. 
The unidentified signals at $9.5 \mathrm{ppm}$ and $2.1 \mathrm{ppm}$ (Figure 20a-VCL+TA@70 ${ }^{\circ} \mathrm{C}, \mathrm{t}-1.5 \mathrm{~h}$ ) correspond to the protons of the aldehyde and methyl group of acetaldehyde. Also, the signal at $1.1 \mathrm{ppm}$ reported as an impurity is present in these results. These confirmed that the monomer VCL is sensitive to hydrolysis under acidic conditions. Nonetheless, the susceptibility to hydrolysis depends on the temperature and reaction time. At room temperature, there is no effect of the tannic acid on the monomer structure. Only at $70{ }^{\circ} \mathrm{C}$, structural variations are observed. In the recipe presented previously (Chapter 2), the VCL/TA-mixture is stirred for 5 minutes at RT, and the addition of the initiator follows immediately after adjusting the temperature to $70{ }^{\circ} \mathrm{C}$. Previous studies in our research group have proved that the polymerization of VCL is a fast reaction, and only 20 minutes are necessary to achieve the maximal conversion. ${ }^{116}$ Therefore, one assumes that only a monomer fraction is hydrolyzed at the initiation time. There are still monomer molecules available for the polymerization so that polymer-based microgels can be synthesized. The hydrolysis process continues during the propagation, explaining the decrease of the conversion rate. This founding is not only essential for further investigations, but also for future studies related with the synthesis of PVCL-based materials. They complement the previous studies in this field and offer a better understanding about the polymerization of VCL.

Accordingly to this discovery, the microgel synthesis was optimized by a semi-batch procedure to incorporate TA during the polymerization. The order of the synthesis steps was modified according to Figure 21.

1.) Polymerization of $\mathrm{VCL}$

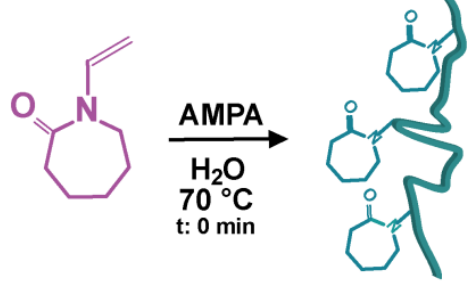

2.) Addition of TA

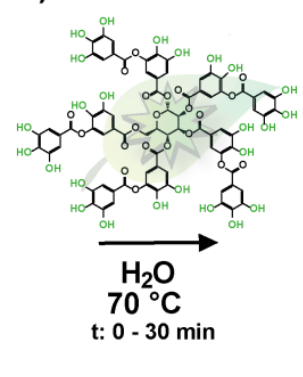

3.) Microgel formation by H-bonding

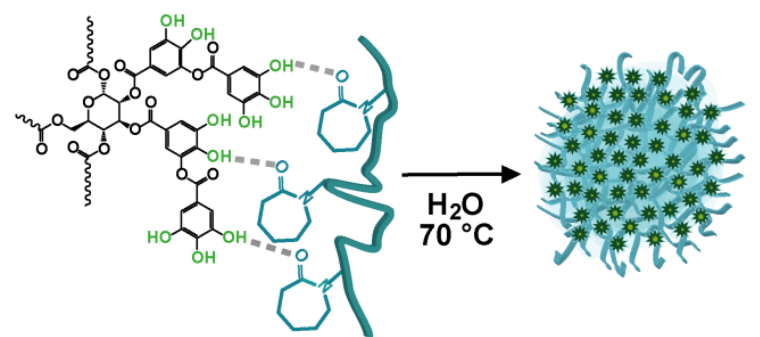

Figure 21. Scheme for the optimized reaction synthesis of the PVCL/TA-microgels.

At first, the initiator was added to the monomer solution at $70^{\circ} \mathrm{C}$ and the precipitation polymerization started without crosslinker. After certain time period, TA was added to the solution. In the end, the complexation between PVCL and TA took place, leading to 
the formation of the supramolecular microgels. In order to identify the best parameters, the addition type (direct or dropwise) for the incorporation of TA as well as the waiting time between the initiation and the crosslinker addition was varied during the second step. The yield of the different experiments was calculated and the results are displayed in Figure 22a.

a.) Reaction yield vs time:

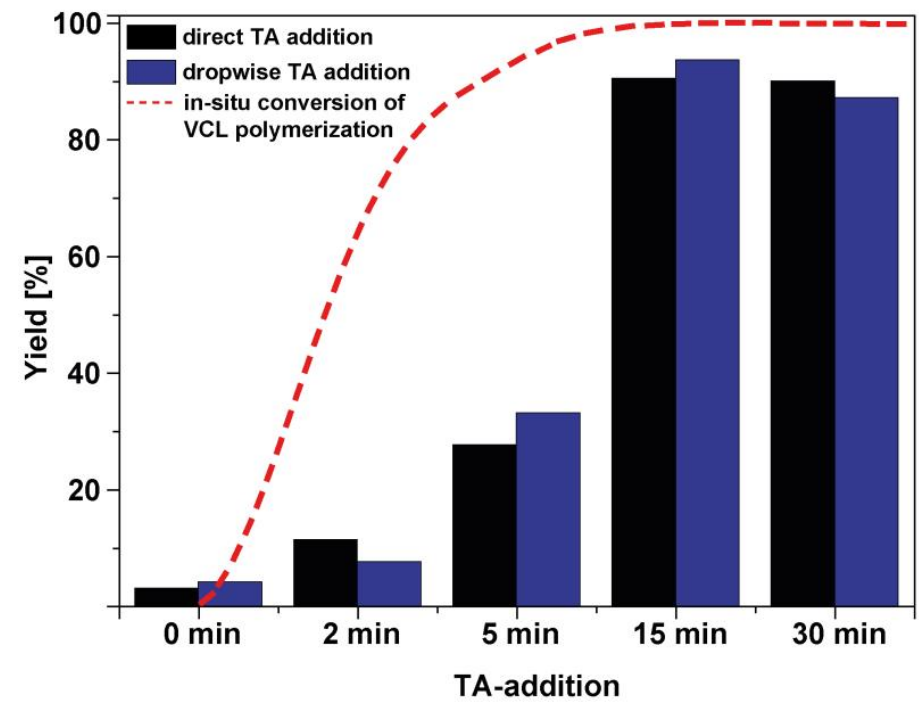

b.) Microgel morphology after t-variation:
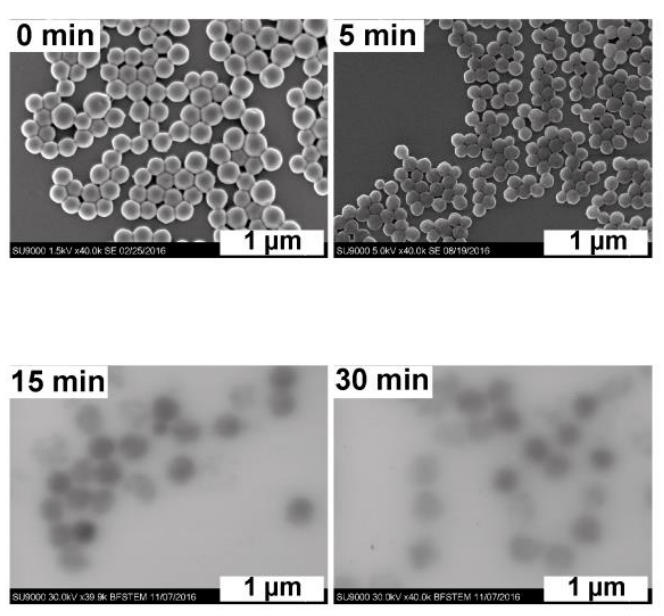

Figure 22. Optimization of the microgel synthesis. a) Yields in dependency of the type of TA-addition and waiting time. b) Effect of the time between initiation and TA-addition on the microgel morphology.

First and foremost, one observes clearly that the type of addition does not influence the conversion or rather the synthesis yields. In both cases (black and blue bars), the results are equivalent. Contrary to this, time seems to play an essential role. A waiting period up to 5 min shows already a minor improvement of the microgel yield (up to $30 \%$ ). However, the addition of TA after15 or 30 min upon the initiation improves the results in a significant manner. Yields of up to $90 \%$ can be reached. The variation of the time affects also the crosslinking density of the materials. The addition of the physical crosslinker at early stages of the polymerization (up to $5 \mathrm{~min}$ ) leads to compact structures. On the other hand, the microgels obtained with a tannic acid addition after 15 min or longer show a rather low crosslinking density (Figure 22b). Microgel softness is expected, due to the fact that only a small amount of crosslinker $(0.5 \mathrm{~mol} \%)$ was incorporated. These results correlate very well to the kinetic study of the VCL polymerization. The conversion of VCL in dependency of the time during a precipitation 
polymerization (without crosslinker) at $70{ }^{\circ} \mathrm{C}$ was monitored in-situ. The dashed curve represents the conversion changes as a function of the time (Figure 22a).

a.) Particle size and polydispersity:

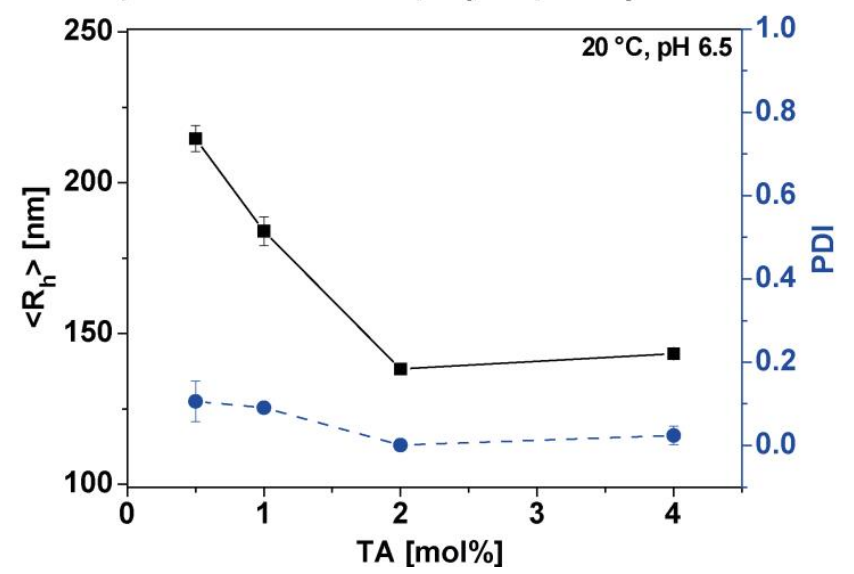

b.) Microgel morphology in dried state:
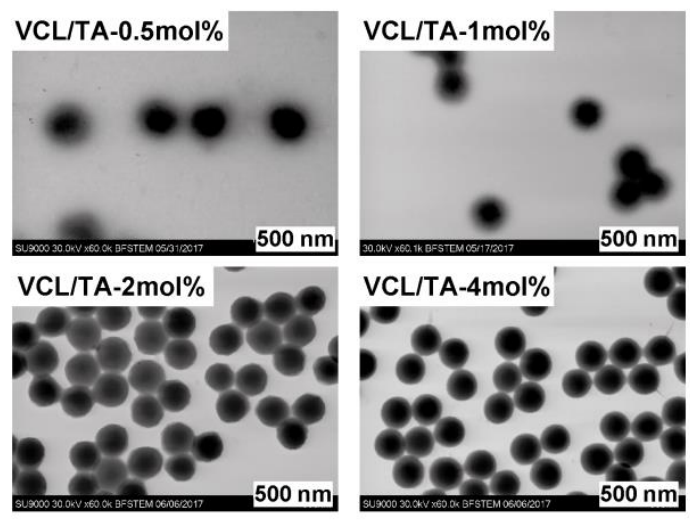

Figure 23. Study of the morphology and dimension of the optimized PVCL/TA-microgels in dependency of the TA-content. a) Particle size, PDI values as well as c) STEM images.

A strong correlation between the VCL conversion and the yields in the presence of TA as a crosslinker can be observed. The addition of TA at late stages of the polymerization leads to a higher microgel production. At this point, the monomer units are almost completely polymerized so that their hydrolysis cannot take place. Only the polymer chains interact with the polyphenol forming stable colloidal and supramolecular gels. In conclusion, the addition of TA was carried out directly 15 min after the initiation for all the further experiments.

After the determination of the optimal synthesis parameters, we analyzed the effect of the VCL-to-TA-ratio in view of microgel morphology and dimension. $\mathrm{R}_{\mathrm{h}}$ and PDI of each system were calculated by DLS measurements. The results as a function of the TAcontent are displayed in Figure 23a. From these investigations, one observes clearly that the higher the TA-amount within the microgels is, the smaller microgel radii become. The STEM-images of the various microgels confirm these results. The increase of the crosslinker-content leads to a stronger crosslinking density and thus, to harder and collapsed microgels. In the case of the systems with 0.5 and $1 \mathrm{~mol} \%$ of TA, the microgel shape is diffuse and not as well defined as in the microgels with higher amounts of crosslinker. However, there is no significant difference between the last both samples (2 and $4 \mathrm{~mol} \%$ ) in terms of particle size. They exhibit a radius of approx. $140 \mathrm{~nm}$ in 
aqueous solution as well as in the dried state. Steric effects between the polymer chains and the TA molecules could limit the total incorporation of the crosslinker.

Derformability of the microgels:
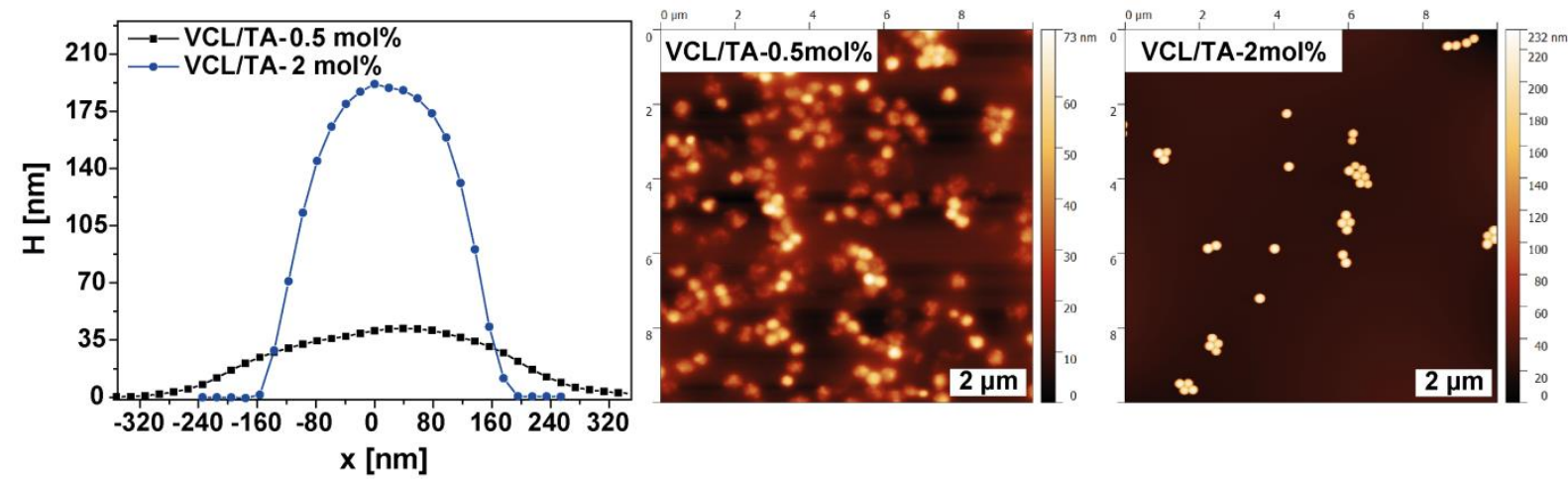

Figure 24. Analysis of PVCL/TA-microgels via AFM.

The deformability of the microgels was analyzed further by atomic force microscopy (AFM) (Figure 24). Not only the cross-sectional profiles, but also the AFM images correlate with the DLS and STEM study. Thus, the increment of the TA-content contributes to stiffness in the microgel network. Once more, the system VCL/TA$0.5 \mathrm{~mol} \%$ shows diffuse contours and its cross-sectional profile indicates spreading of the soft microgels on the surface. Opposite to this, well-defined and rigid particles are obtained with 2 mol\% of TA. The microgels do not deform and remain hard. This analysis demonstrates explicitly that the structural properties of our materials can be tuned in a controlled and easy manner only by varying the polyphenol amount.

a.) Size by varied surfactant amount:

b.) Microgel morphology after surfactant variation:
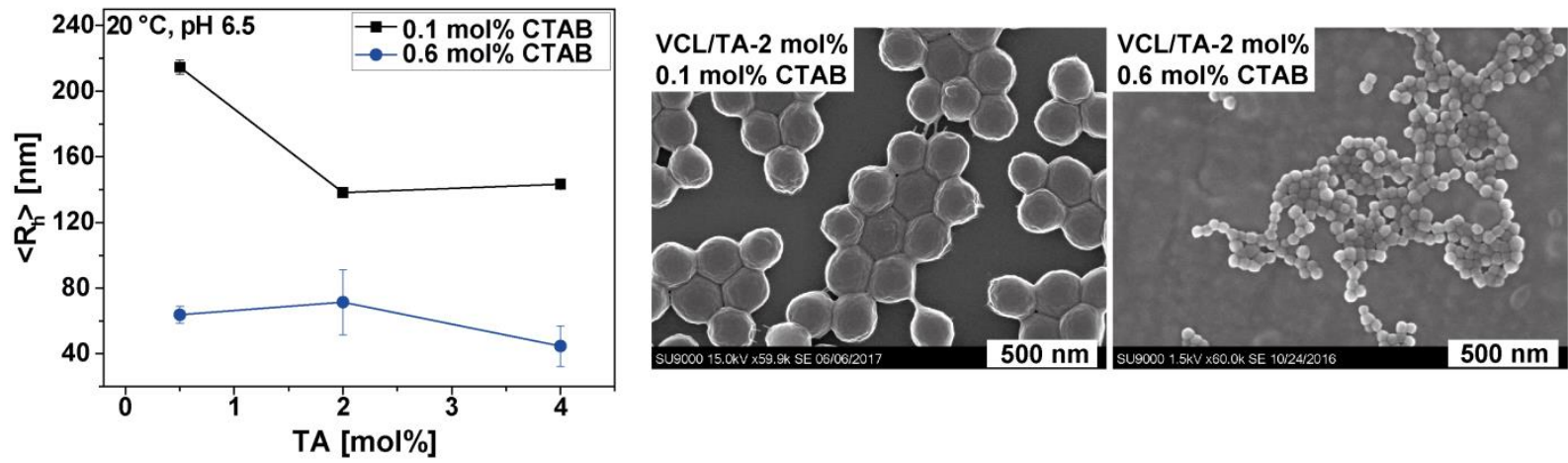

Figure 25. Influence of the surfactant amount used during the microgel synthesis.

Further experiments were performed to analyze the additional effect of the surfactant concentration. According to the preliminary microgel synthesis, CTAB was used as surfactant during the optimized approach. This stabilizing agent is incorporated in small 
quantities to the reaction before the initiation. The influence of this additional substance was tested by a variation of its molar amount (0.1 and $0.6 \mathrm{~mol} \%)$ and the obtained synthesis results are displayed in Figure 25. One can confirm clearly that increasing the surfactant content during the microgel formation, reduces the hydrodynamic radii of the synthesized microgels (Figure 25a). The microgel size decrease can be confirmed by additional microscopic measurements. The STEM-images (Figure 25b) show the noticeable reduction of the microgel diameter, pointing out the variation of the surfactant amount as an interesting tool to produce microgels in a broad dimensional scale.

a.) Raman-spectra

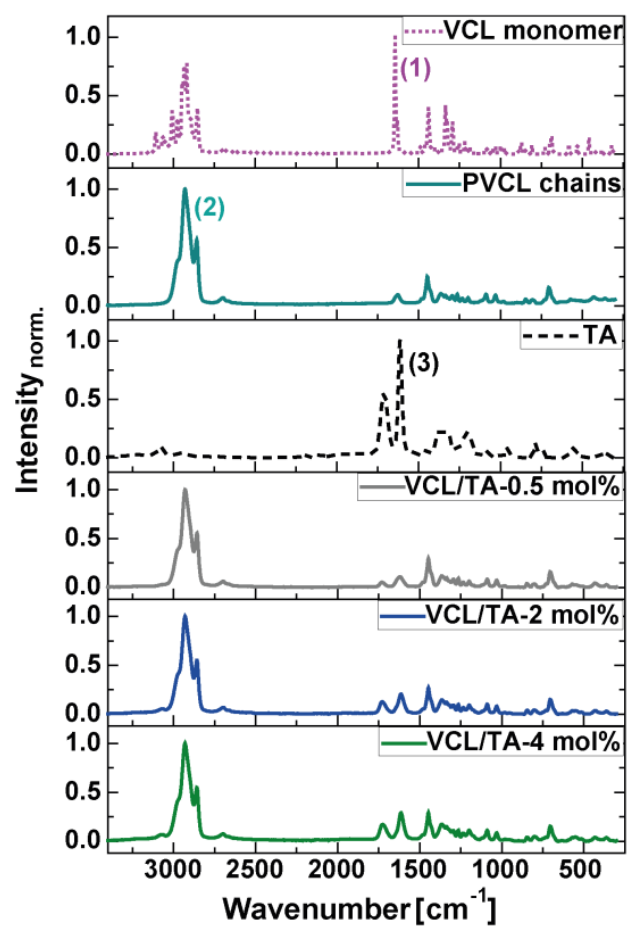

b.) Molecular structures:

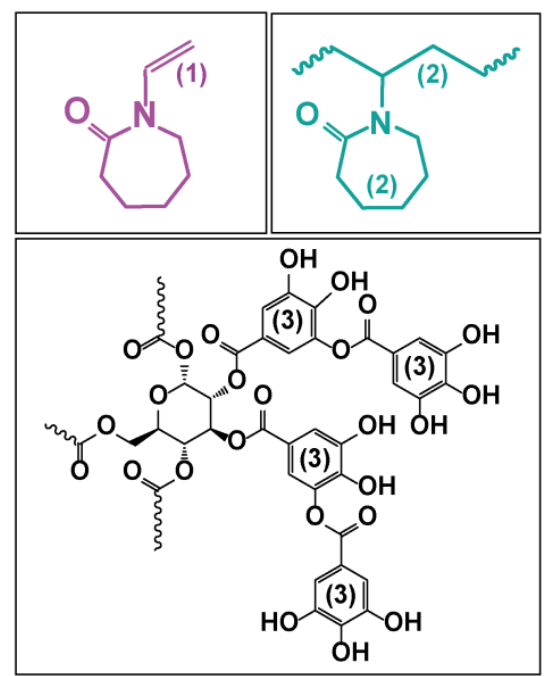

Figure 26. Raman measurements for the analysis of the chemical composition of the PVCL/TA-microgels.

Moreover, the incorporation of the TA into the microgels was confirmed by Raman spectroscopy. The spectra of the microgels with various TA-contents in comparison to the references (VCL: monomer, PVCL: polymer chains and TA: crosslinker) are depicted in Figure 26. In all PVCL/TA-samples the relevant signals for the PVCL and TA can be identified, and more specifically, it can be noticed that the increment of the crosslinker amount leads to an expected moderate increase of the signal intensity. The real amount of TA within the microgels was quantified (Table 6). 
Table 6. Real TA content of the supramolecular microgels quantified by Raman.

\begin{tabular}{|c|c|c|}
\hline Microgel & TA $_{\text {theo. }}[\mathrm{mol}-\%]$ & TA $_{\text {real }}[\mathrm{mol}-\%]$ \\
\hline VCL/TA-0.5mol\% & 0.5 & 0.50 \\
\hline$V C L / T A-1 m o l \%$ & 1 & 0.83 \\
\hline$V C L / T A-2 m o l \%$ & 2 & 1.29 \\
\hline$V C L / T A-4 m o l \%$ & 4 & 1.68 \\
\hline
\end{tabular}

The calculated values follow the expected trend with the expectations, even though, they do not correspond exactly to the theoretical amounts. As mentioned before, steric effects could be the reason for a limited incorporation of TA into the polymer network. This remark correlates also with the preliminary observations obtained by ITC (Chapter 2, Figure 6). However, the comparison of this study to the first proposed synthesis procedure confirms that the optimized route is more accurate and suitable for a controlled microgel design and composition.

Thermal stability of supramolecular PVCL/TA-microgels:
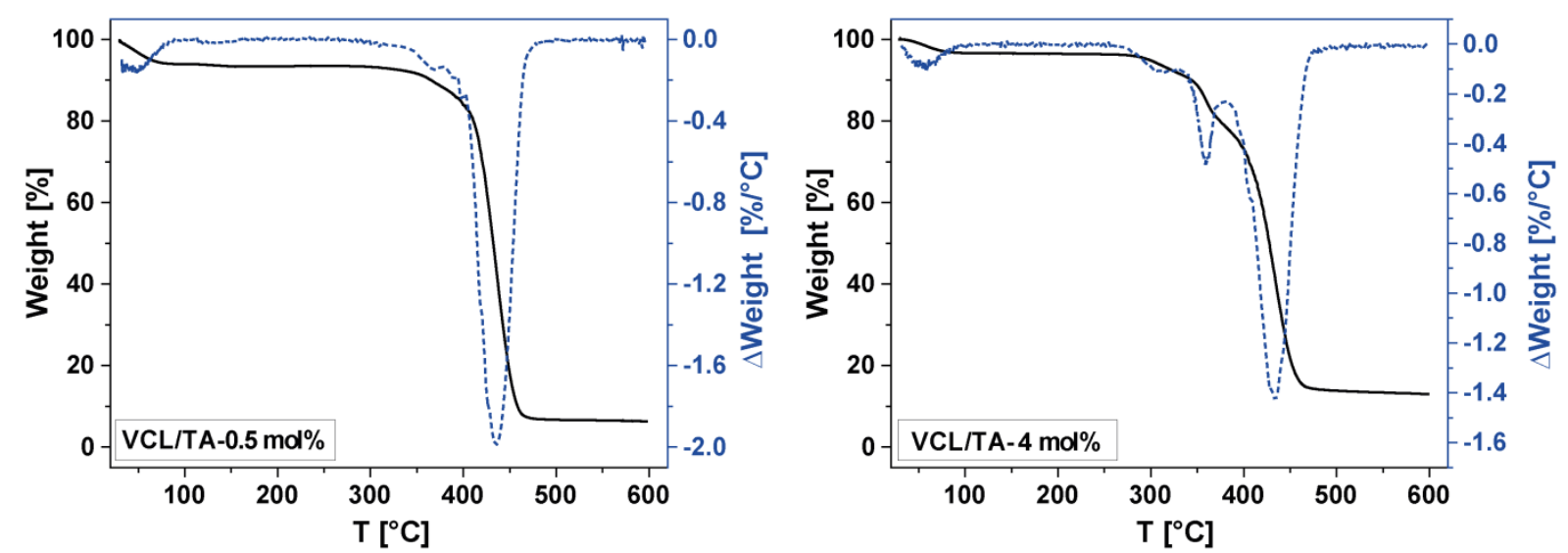

Figure 27. Characterization of the thermal degradation of PVCL/TA-microgels by TGA.

Besides the structural characterization, the thermal stability of the supramolecular microgels was analyzed by thermal gravimetric analysis (TGA) and the results are depicted in Figure 27. The obtained TGA curves reveal a thermal stability of up to $400{ }^{\circ} \mathrm{C}$ for the supramolecular microgels and confirm the presence of the physical crosslinker TA in different fractions. Table 7 summarized the experimental degradation temperatures of the references and the PVCL/TA-microgels as complementary information. 
Table 7. Degradation temperatures of VCL-monomer and Ta determined by TGA.

\begin{tabular}{|c|c|}
\hline Substance & $\mathbf{T}_{\text {deg }}\left[{ }^{\circ} \mathbf{C}\right](\Delta$ weight $\%)$ \\
\hline Covalent PVCL microgels & $444.2(96.2 \%)$ \\
\hline TA & $69.1(6.0 \%) ; 264.7$ and $320.0(53.6 \%)$ \\
\hline VCL/TA-0.5mol\% theo. & $50.4(5.4 \%) ; 367.6(5.5 \%) ; 437.1(79.7 \%)$ \\
\hline VCL/TA-4mol\% theo. & $57.9(3.2 \%) ; 304.8(4.7 \%) ; 359.4(13.0 \%) ; 433.3(64.4 \%)$ \\
\hline
\end{tabular}

In the case of TA as well as the TA-based microgels, the signals at a temperature between 50 and $70{ }^{\circ} \mathrm{C}$ represent a weight loss of approximately 5 to $6 \%$ and can be assigned to the evaporation of residual water in the materials, as well as the start of TA oxidation. Moreover, the thermal domains between 250 and $370{ }^{\circ} \mathrm{C}$ correspond to further oxidative processes of TA and also its different depolymerization steps. ${ }^{117}$ Finally, the largest weight change for both PVCL/TA-microgels occurs at a temperature between $430-450{ }^{\circ} \mathrm{C}$. This peak represents a mass loss of almost $80 \%$ (VCL/TA$0.5 \mathrm{~mol} \%$ theo.) and 65\% (VCL/TA-4mol\%theo.) in each sample, and relates to the complete disintegration of the polymeric chains. Rests of carbon can be found in all TA-based systems including the pure substance at a temperature of $600{ }^{\circ} \mathrm{C}$, representing a mass residue between 30 and $10 \%$.

Related to the microgel stability, solutions of a PVCL/TA-microgel were prepared in water and various organic media: tetrahydrofuran (THF), chloroform $\left(\mathrm{CHCl}_{3}\right)$ and methanol $(\mathrm{MeOH})$, to analyze their behavior. The influence of each solvent was characterized via electron microscopy (Figure 28). Normally, VCL-based microgels are amphiphilic very stable in different organic solutions due to the strength of their covalent-crosslinks. In contrary, the utilization of physical crosslinkers in microgel design leads to higher sensitivity upon environmental changes since the non-covalent crosslinking is based on a cooperative mechanism of weak forces. The type of solvent has therefore a significant role in view of microgel stability. 


\section{Microgel stability in solution:}

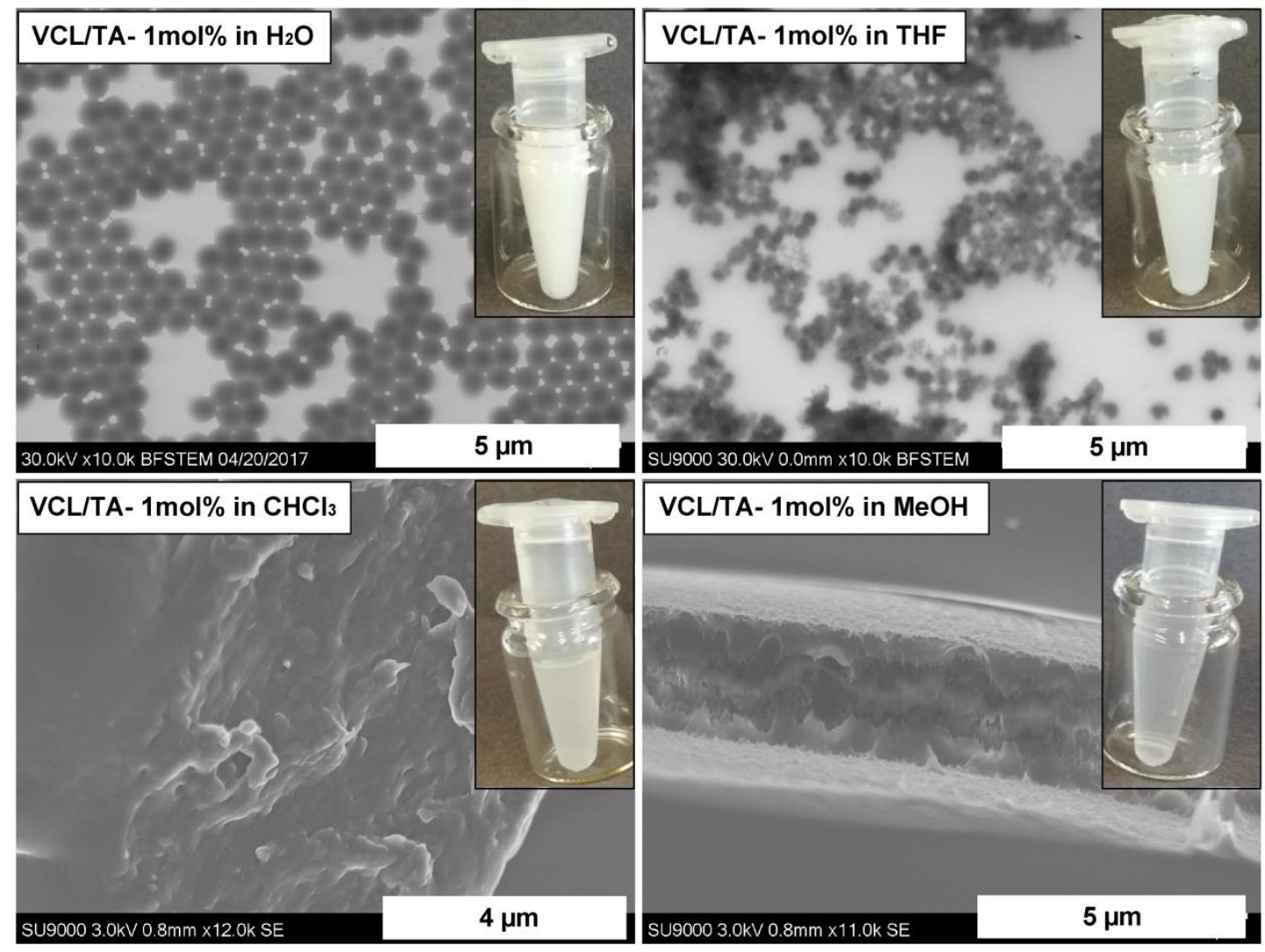

Figure 28. Stability of PVCL/TA-microgels in different solvents.

In this experiment, the PVCL/TA-microgels showed high stability in $\mathrm{H}_{2} \mathrm{O}$ and THF. However, their dissolution in $\mathrm{CHCl}_{3}$ and $\mathrm{MeOH}$ led to a disintegration of the well-defined microgel particles. As observed previously, the microgels are water-based materials. The presence of $\mathrm{H}_{2} \mathrm{O}$ within the polymeric network does not interfere between the polymer chains and TA, even though $\mathrm{H}_{2} \mathrm{O}$ is a hydrogen donor due to its protic type. The VCL-toTA hydrogen bonding is still stronger and remains stable. Moreover in the case of THF, one can concluded that its aprotic property promote the stability of the microgels. In contrary, the presence of $\mathrm{CHCl}_{3}$ leads to a re-assembly of the system and the formation of a gel. The insolubility of TA in $\mathrm{CHCl}_{3}$ could be one possible reason for the leaching of TA and the reorganization of the material structure. Lastly, the dissolution of the supramolecular microgels in $\mathrm{MeOH}$ showed similar results. Herein, the protic property of the alcohol to form hydrogen bonds can explain the formation of a macrogel in order to achieve an energetic favorable ordered state. For a better understanding of these results, further analysis methods have to be applied. The determination of the binding 
constant between the building blocks and the solvents, as well as the position within the microgels would explain the re-assembly mechanism.

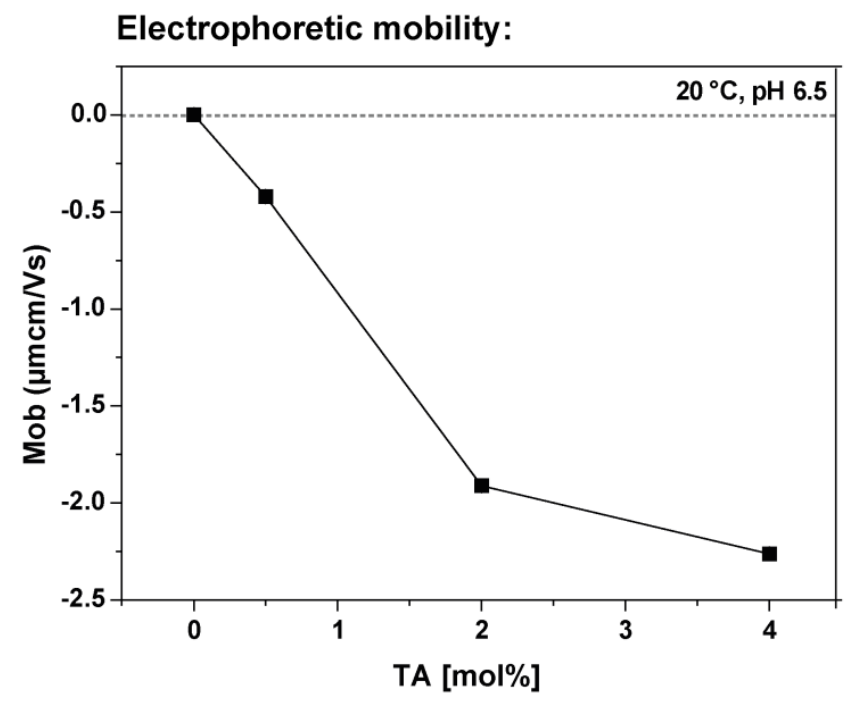

Figure 29. Study of the microgel electrophoresis.

The electric surface charge of the PVCL/TA-microgels was studied by electrophoretic mobility measurements (Figure 29). Like the morphology and dimension of the materials, this property can be tuned in dependency of the TA-amount. The higher the crosslinker content, the more hydroxy-groups are incorporated within the microgels and therefore the more negative charged they are.

a.) Thermoresponsiveness:

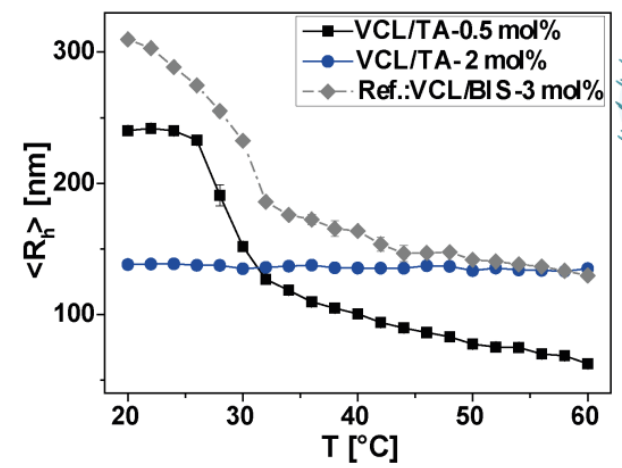

b.) Water up-take capacity:
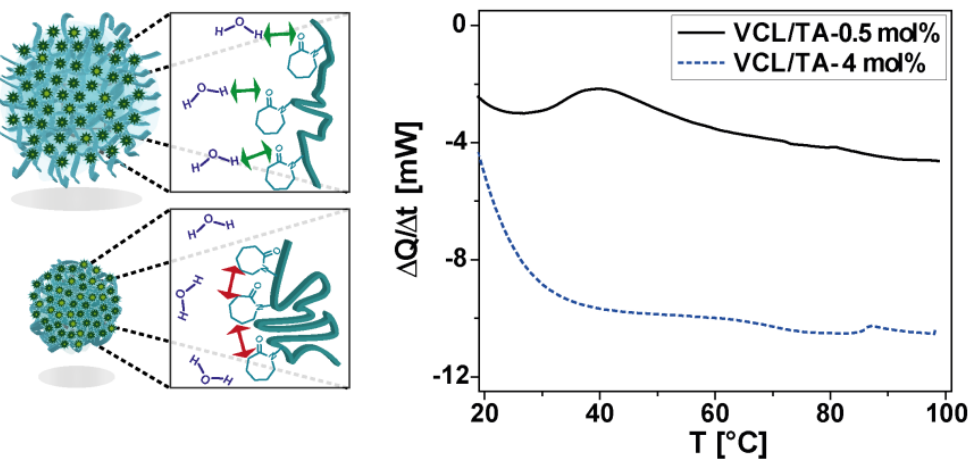

Figure 30. a) DLS-study of the thermoresponsiveness of the optimized PVCL/TA-microgels and b) complementary analysis of the water up-take capacity via DSC.

In addition, the thermal sensitivity of the PVCL/TA-materials was characterized. PVCL as well as PVCL-based systems are well-known as thermoresponsive materials. In our preliminary studies, it was demonstrated that the swelling/deswelling of the microgels is not only dependent of the temperature, but can also be hindered depending on the 
TA-amount. 115 The optimized PVCL/TA-microgels behave analog (Figure 30a). Microgels with low TA-percentage $(0.5 \mathrm{~mol} \%)$ are less densely packed and can interact strongly with water molecules by H-bonding. This leads to a strong water uptake and a high degree of swelling/deswelling. By increasing the environmental temperature, the polymer-to-polymer interactions become stronger and the microgels collapse. Nevertheless; the increase of the TA-content up to $2 \mathrm{~mol} \%$ inhibits the thermal properties. In this case, the physically crosslinked microgels already show a compact and rigid morphology because of the high crosslinking density. The polymer segments interact more intensely with the tannic acid forming strong H-bonds. For this reason, the interactions with the water molecules are weak and there is no reversible and temperature dependent swelling to be observed.

The DLS measurements correlate furthermore with the results obtained by differential scanning calorimetry (DSC). The DSC curves of the PVCL/TA-microgels treated with $\mathrm{D}_{2} \mathrm{O}$ are represented in Figure 30b. Herein, the supramolecular microgels with less TAamount (black curve) exhibit a volume phase transition reflected by a broad signal at $40{ }^{\circ} \mathrm{C}$. By DLS, a VPTT of approximately $30^{\circ} \mathrm{C}$ was determined for this microgel. Still, the shift of the signal to higher temperatures could be explain due to the use of $\mathrm{D}_{2} \mathrm{O}$ instead of $\mathrm{H}_{2} \mathrm{O}$ during this approach. ${ }^{118}$ In comparison, the sample with a higher TA-fraction do not show any water up-take capacity and confirms the conclusions discussed above.

\section{pH-stability of the optimized supramolecular microgels:}
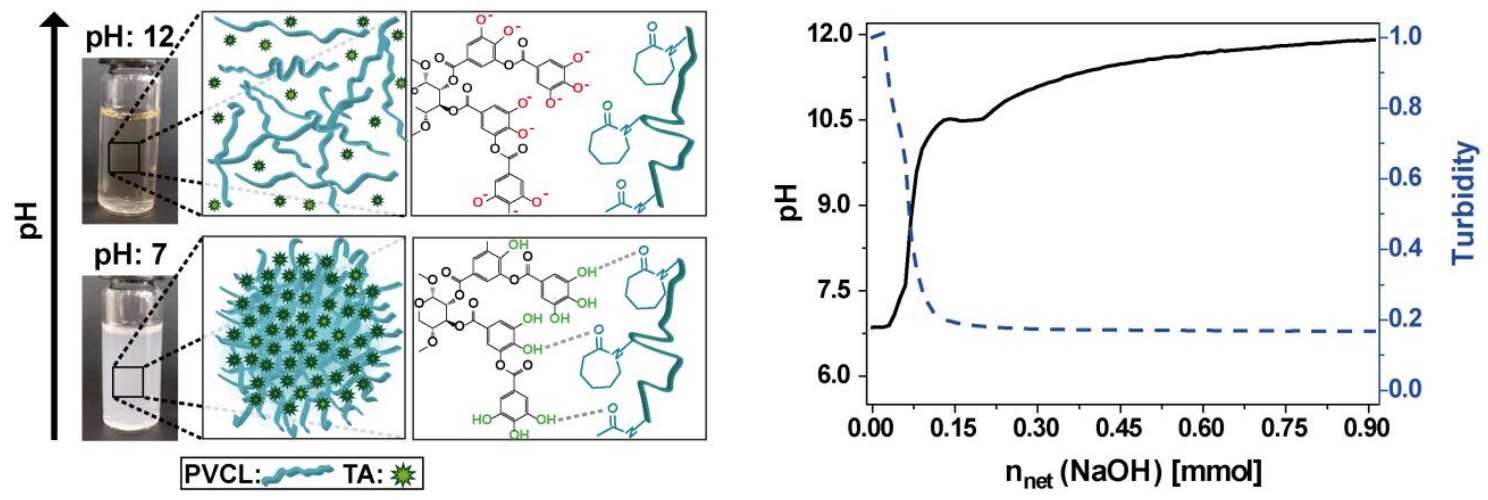

Figure 31. Influence of the medium pH on the stability of the optimized PVCL/TA-microgels.

The supramolecular nature of the optimized PVCL/TA-microgels and their pH-controlled disassociation was also studied subsequently. Hereby, a microgel solution

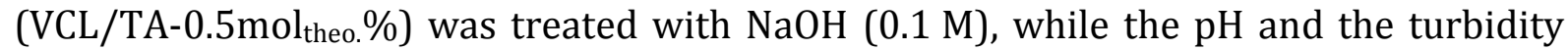


were monitored (Figure 31). Similar to our preliminary systems (Chapter 2), the novel microgels behave stable at neutral conditions and their disassembly occurs after reaching a $\mathrm{pH}$ of 8.5. At this point, the turbid solution becomes clear and yellowish indicating the release of the TA molecules because of deprotonation of their hydroxyl-groups, and the disintegration of the microgel network due to the breaking of the H-bonds. With this result, the supramolecular formation of the microgels can be emphasized once more.

Lastly, a further synthesis approach was studied for the production of supramolecular microgels using already synthesized linear PVCL chains with a defined molecular weight. This route is illustrated in Figure 32.

1.) PVCL collapse

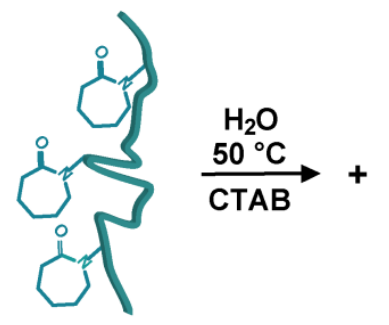

2.) Addition of TA

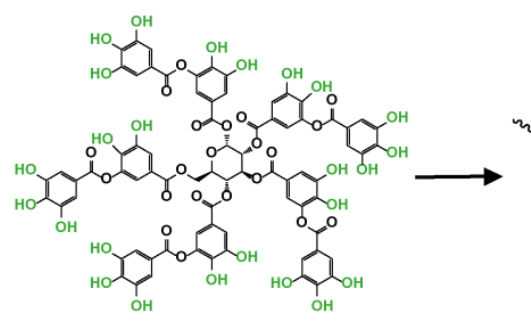

3.) supramolecular interactions

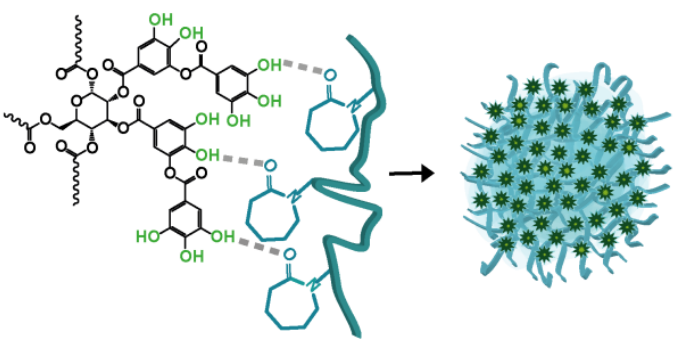

Figure 32. Illustrated synthesis route of the microgel production from linear PVCL chains with specific molecular weights.

In our previous synthesis procedures, the microgel production was carried out during the precipitation polymerization of VCL. However, this method relays on a free radical polymerization mechanism leading to different lengths for the polymeric chains. ${ }^{119}$ In order to improve the control of the microgel structure and achieve a more specific polymer network, PVCL with a determined chain length was proposed as precursor. The different PVCL samples used for this new proposed synthesis were polymerized previously to the microgel synthesis by reversible addition-fragmentation chain transfer (RAFT), a controlled radical polymerization method. After the RAFT-polymerization PVCL, the material was purified and the molecular weight was characterized by GPC. Finally, the microgel synthesis took place. In this case, the polymeric chains were dissolved in water in the presence of a surfactant (CTAB). Subsequently, the PVCLsolution was heated up to $50^{\circ} \mathrm{C}$ to reach the collapsed state of the thermoresponsive polymer. At this point, the crosslinker TA was added to the solution and the mixture was 
stirred for $30 \mathrm{~min}$ at the same temperature. In the end, the solution was cooled down and the product was purified by dialysis.

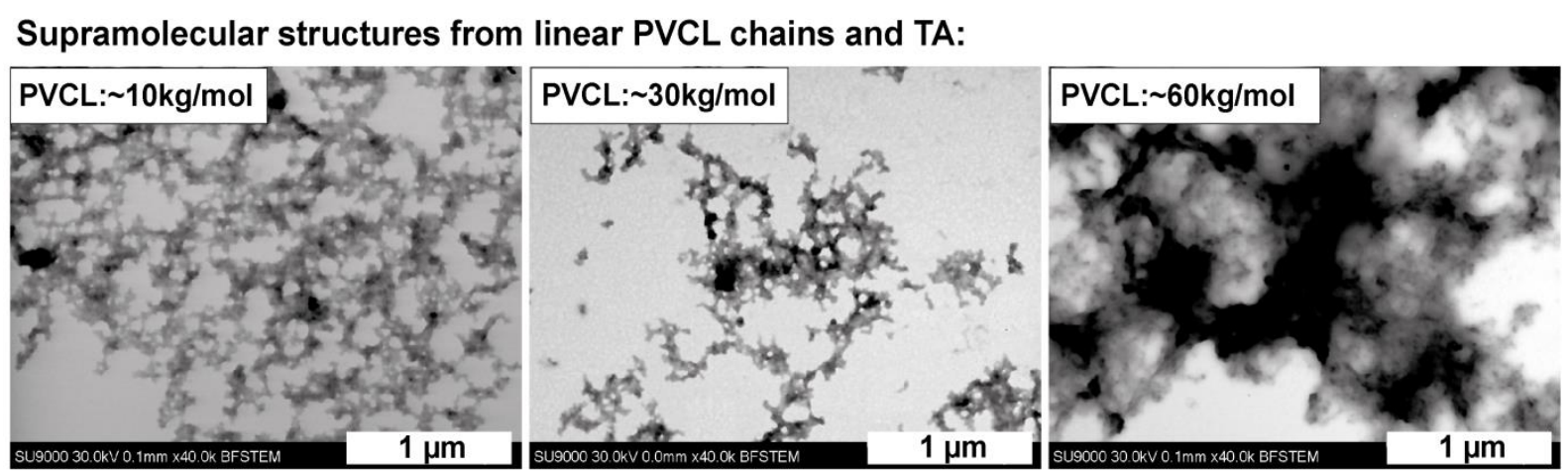

Figure 33. STEM images of supramolecular products obtained by the synthesis of microgels using defined PVCL chains as precursors.

The morphology of the supramolecular product and the possible microgel formation was analyzed by STEM (Figure 33). Unfortunately, the microscopy images do not show the expected results. The synthesis procedure was performed using 3 different PVCL samples $\left(\overline{\mathrm{M}}_{\mathrm{n}}: 10000 \mathrm{~g} \cdot \mathrm{mol}^{-1}, 30000 \mathrm{~g} \cdot \mathrm{mol}^{-1}\right.$ and $\left.60000 \mathrm{~g} \cdot \mathrm{mol}^{-1}\right)$. However, none of the different specific lengths led to the formation of stable microgels. It can be assumed that longer chains are necessary for the production of stable microgel particles. This should be confirmed by further studies using higher molecular weights as well as different polymers.

Nevertheless, the optimized synthesis route (page Fehler! Textmarke nicht definiert., Figure 21) provides already an easy and optimized procedure, which does not imply multiple and difficult steps. This approach allows not only the production of self-assembled microgels, but also offers numerous possibilities to vary the material properties in a systematic form.

\subsection{Conclusions}

In the present section, the optimization of novel supramolecular and stimuli responsive microgels is reported. Based on the initial results related to the microgel synthesis and the low obtained yields, the stability of the monomer VCL was studied in the presence of TA. The effect of the $\mathrm{pH}$ and temperature on the microgel synthesis was therefore determined in this work. We confirmed not only that the monomer VCL is sensitive to hydrolysis under acidic conditions, but also, we discovered that this process is 
temperature and time dependent. This result complements the studies of Imaz et. al., and can be substantial for other investigations in view of novel PVCL-based materials. This discovery helped to modify the initial microgel synthesis and generated an improved method. The optimized PVCL/TA-microgels showed tunable structural and responsive properties. In particular, we demonstrated the supramolecular nature of our materials by their pH-triggered disassembly. This relevant property confirmed the successful formation of $\mathrm{H}$-bonds between the polymer chains and the tannic acid molecules. Because of the importance of spontaneous organization in view of production of bio-inspired systems, the non-covalently crosslinked PVCL/TA-microgels could be attractive for biomedical applications among other fields, in which responsiveness plays an essential role. 


\subsection{Experimental Section}

\subsubsection{Materials}

2,2'-Azobis-(2-methylpropionamidine) dihydrochloride (AMPA, 97\%, Aldrich), cetyltrimethylammonium bromide (CTAB, $\geq 97.0 \%$, Merck Millipore), tannic acid (TA, Sigma-Aldrich), sodium hydroxide solution (0.1 N, Sigma-Aldrich), $\mathrm{D}_{2} \mathrm{O}$ (99.9 atom \% D, Sigma-Aldrich), and organic solvents ( $\left.\mathrm{MeOH}, \mathrm{CHCl}_{3}, \mathrm{THF}, \mathrm{EtOH}, \mathrm{DMSO}\right)$, and dialysis membranes (Zellu Trans/Roth MWCO 3500, Carl Roth) were used without further treatment. $N$-vinylcaprolactam (VCL, $98 \%$, Aldrich) was distillated under vacuum before use. Linear PVCL was synthesized via RAFT polymerization by Chaolei Hu (Research group of Prof. Andrij Pich, DWI-Leibniz-Institute for Interactive Materials). The polymers were purified by dialysis. Deionized water was used for all experiments.

\subsubsection{Yield and Conversion Studies}

\section{a) Yield Determination by Gravimetric Analysis}

Certain amount (1/30) of microgel solution was freeze dried after purification, and the mass of the product was calculated gravimetrically (triple determination). The yield values were determined from the quotient between the theoretical and real masses.

$$
\begin{aligned}
& \text { (1) } m_{\text {theo }}=m_{\text {monomer }}+m_{\text {crosslinker }}+m_{\text {initiator }} \\
& \text { (2) } \bar{m}_{\text {real }}=\frac{\Sigma\left(m_{\text {product }} \cdot 30\right)}{3} \\
& \text { (3) Yield }=\frac{\bar{m}_{\text {real }}}{m_{\text {theo }}} \cdot 100 \%
\end{aligned}
$$

\section{b) Study of the Monomer Stability by Nuclear Magnetic Resonance Spectroscopy}

For the study of the stability of VCL in the presence of tannic acid $(\mathrm{pH}=4)$, the monomer (1 g, $139.2 \mathrm{~g} \cdot \mathrm{mol}-1)$ was dissolved in deionized water $(10 \mathrm{~mL})$. The temperature was adjusted (room temperature or $\left.70^{\circ} \mathrm{C}\right)$ and TA $(0.5 \mathrm{~mol} \%)$ was added to the solution under stirring. Samples were withdrawn at different times and characterized by ${ }^{1} \mathrm{H}$ NMR. The measurements were performed on a Bruker DPX-400 FT-NMR Spectrometer at $400 \mathrm{MHz}$ in $\mathrm{D}_{2} \mathrm{O}$ at room temperature. 


\section{c) Conversion Calculation via Calorimetric Measurements}

The conversion of VCL by precipitation polymerization (without crosslinker) was calculated in-situ by calorimetry. The measurement was carried out in the isothermal mode. on a calorimeter RC1e (Mettler Toledo) interconnected to a $500 \mathrm{~mL}$ 3-wall AP010.5-RTCal reactor with a Hastelloy® stirrer, a baffle and a Turbido ${ }^{\mathrm{TM}}$ turbidity probe (Solvias). Using the software iControl RC1et 5.0, the data was analyzed. The conversion values were calculated by the integration of the reaction heat curve.

\subsubsection{Microgel Synthesis}

\section{a) Route II: Synthesis of PVCL/TA-Microgels by Semi-Batch Precipitation Polymerization}

The optimized production of the PVCL/TA-microgels was carried out via precipitation polymerization in a semi-batch procedure. In a glass reaction flask $(250 \mathrm{~mL}$ total volume), the monomer VCL $\left(2 \mathrm{~g}, 139.2 \mathrm{~g} \cdot \mathrm{mol}^{-1}\right)$ and the surfactant CTAB $(0.006 \mathrm{~g}, 364.45$ $\left.\mathrm{g} \cdot \mathrm{mol}^{-1}\right)$ were dissolved in deionized water $(150 \mathrm{~mL})$ under room conditions. The solution was stirred 30 min under $\mathrm{N}_{2}$-atmosphere, and subsequently the temperature of the reaction was adjusted to $70{ }^{\circ} \mathrm{C}$. By the addition of AMPA (0.05 g, $\left.271.19 \mathrm{~g} \cdot \mathrm{mol}^{-1}\right)$, the polymerization was started. Finally after certain time period (0-30 min), the crosslinker TA $\left(0.247\right.$ - $1.019 \mathrm{~g}, 1701.2 \mathrm{~g} \cdot \mathrm{mol}^{-1}$, Table 8) was added to the solution. The polymerization was stopped $1 \mathrm{~h}$ after the initiation. The product was purified by dialysis in deionized water for 4 days, and freeze dried.

Influence of surfactant: The effect of the utilization of a cationic surfactant during the synthesis of the PVCL/TA-microgels was analysed. Herein, the synthesis of all samples was repeated with increased CTAB-amount (0.030 g).

Table 8. Hydrodynamic radii, PDIs and yields of the optimized PVCL/TA-microgels.

\begin{tabular}{|c|c|c|c|c|}
\hline Microgel & $\mathrm{TA}[\mathrm{g}]$ & $<_{\mathrm{h}-20^{\circ} \mathrm{C}}>[\mathrm{nm}]$ & $\mathbf{P D I}_{20^{\circ} \mathrm{C}}$ & Yield [\%] \\
\hline VCL/TA-0.5mol\% & 0.122 & $214.6 \pm 4.3$ & $0.106 \pm 0.049$ & 96.9 \\
\hline VCL/TA-1mol\% & 0.247 & $183.9 \pm 4.7$ & $0.091 \pm 0.013$ & 97.5 \\
\hline VCL/TA-2mol\% & 0.509 & $138.2 \pm 0.0$ & $0.001 \pm 0.0$ & 96.1 \\
\hline VCL/TA-4mol\% & 1.019 & $143.3 \pm 1.8$ & $0.024 \pm 0.022$ & 90.1 \\
\hline
\end{tabular}




\begin{tabular}{|c|c|c|c|c|}
\hline $\operatorname{Microgel}(5 x C T A B)$ & TA[g] & $<\mathrm{R}_{\mathrm{h}-20^{\circ} \mathrm{C}}>[\mathrm{nm}]$ & $\mathrm{PDI}_{20^{\circ} \mathrm{C}}$ & Yield [\%] \\
\hline VCL/TA-0.5mol\%-B & 0.122 & $63.8 \pm 5.1$ & $0.204 \pm 0.056$ & 96.9 \\
\hline VCL/TA-2mol\%-B & 0.509 & $71.4 \pm 19.7$ & $0.250 \pm 0.032$ & 96.1 \\
\hline VCL/TA-4mol\%-B & 1.019 & $44.7 \pm 12.3$ & $0.309 \pm 0.038$ & 90.1 \\
\hline
\end{tabular}

\section{b) Route III: Synthesis of PVCL/TA-Microgels based on Linear PVCL Chains by Batch Precipitation Polymerization}

The synthesis of PVCL/TA-microgels using linear PVCL chains as precursor was performed in a semi-batch approach. Previous to the microgel synthesis, samples of PVCL with different specific molecular weights were synthesized via RAFT polymerization by Chaolei Hu. The polymers obtained were purified by dialysis and characterized by GPC to determine the molecular weights.

PVCL $(0.1335 \mathrm{~g})$ and the surfactant CTAB $\left(0.0004 \mathrm{~g}, 364.45 \mathrm{~g} \cdot \mathrm{mol}^{-1}\right)$ were dissolved in deionized water $(10 \mathrm{~mL})$ in a glass reaction flask (100 $\mathrm{mL}$ total volume) and stirred for $30 \mathrm{~min}$ under room conditions. Subsequently, the reaction solution was heated up to $50{ }^{\circ} \mathrm{C}$ and stirred for $10 \mathrm{~min}$ to ensure equilibrium. TA (variation of amount, $1701.2 \mathrm{~g} \cdot \mathrm{mol}^{-1}$ ) was added to the solution with a syringe and the reaction was performed for $30 \mathrm{~min}$. After cooling down the product, it was purified by dialysis for seven days in deionized water.

\subsubsection{Characterization Methods}

\section{a) Dynamic Light Scattering Measurements}

The microgel size/size distribution was characterized by DLS using ALV/CGS-3 Compact Goniometer System from the company ALV-GmbH with an ALV/LSE 5004 tau digital correlator and a JDS uniphase laser operating at $632.8 \mathrm{~nm}$. The scattering angle was fixed $\left(\theta=90^{\circ}\right)$ for all measurements. The temperature dependent experiments were performed in a range from 20 to $60^{\circ} \mathrm{C}$. All samples were filtered before the measurements with Chromafil@ Xtra syringe filters $(0.45$ or $1.20 \mu \mathrm{m})$. The results were obtained by ALV-Correlator Software V3.0. 


\section{b) Electrophoretic Mobility Measurements}

The surface charge of the PVCL/TA-microgels was determined by measurements of the electrophoretic mobility (Mob) with a Zetasizer NanoZS (Malvern). All microgels were measured at $20^{\circ} \mathrm{C}$ and neutral pH ( 6.5) after dispersing them in HPLC-grade water.

\section{c) Scanning Transmission Electron Microscopy}

The morphology of the microgels was studied by SEM and TEM on an Ultra-high Resolution Scanning Electron Microscope SU9000 (Hitachi-High Technologies America, Inc.). $100 \mu \mathrm{L}$ of each microgel solution $\left(0.1 \mathrm{~g} \cdot \mathrm{L}^{-1}\right)$ were diluted in $1 \mathrm{~mL}$ deionized water. $20 \mu \mathrm{L}$ of it were dropped on a TEM-grid (Carbon Film 200 Mesh Copper Grids, Electron Microscopy Sciences) and dried at room temperature overnight. The samples were sputtered with $2 \mathrm{~nm}$ carbon before the measurements.

This work was performed by Sabrina Mallmann in part at the Center for Chemical Polymer Technology CPT, which is supported by the EU and the federal state of North Rhine-Westphalia (grant no. EFRE 3000883 02).

\section{d) Atomic Force Microscopy}

The crosslinking density of the supramolecular microgels was characterized by AFM. All samples were prepared via spin coating on Si-wafers. The substrates were activated in a plasma oven (Plasma Technology, 0.2 mbar, $60 \mathrm{~s}$ ). The deposition of microgels (10 $\left.\mathrm{mg} \cdot \mathrm{mL}^{-1}\right)$ on treated surfaces was carried out on a WS-650SZ-6NPP/LITE spin coater with the speed of $2000 \mathrm{rpm}$ for $1 \mathrm{~min}$.

The AFM-measurements were performed by Alexander Töpel on an Agilent 5500 atomic force microscope in tapping mode. Cantilevers with resonance frequencies of 250-300 $\mathrm{kHz}$ and spring constants around $42 \mathrm{~N} \mathrm{~m}^{-1}$ were used. The data was analyzed by Gwyddion software. Spin Coating: Microgel-based Films.

\section{e) Raman Spectroscopy Analysis}

The incorporation of the physical crosslinker TA in to the microgel network was confirmed and quantified by Raman spectroscopy on a Bruker RFS 100/S Raman spectrometer with a Nd:YAG laser (1064 nm). The measurements were carried out with 
a spectral resolution of $14 \mathrm{~cm}^{-1}$, power of $200 \mathrm{~mW}$ and 1000 scans. The results were analyzed using the software OPUS 4.0.

The quantification of the TA content within the microgels was performed using a calibration curve. Therefore, linear PVCL and TA were mixed in specific ratios and homogenized in methanol. The solvent was removed by evaporation at room temperature and the calibration samples were measured by Raman spectroscopy. The obtained results for the calibration and the corresponding graph are displayed in the next section.

\section{f) Potentiometric Titration and Turbidity Measurements}

Aqueous microgel solutions $(0.1$ wt.- $\%)$ were treated with $\mathrm{NaOH}(0.1 \mathrm{M})$ in $100 \mu \mathrm{L}$ steps until a pH of 12 was achieved. The titration curves were measured on a Metrohm Titrando 905.

At the same time the transmission intensity $I_{\text {transm.norm. }}$ in dependency of the $\mathrm{pH}$ was measured using an optrode $(640 \mathrm{~nm})$. The obtained data were normalized and related to the turbidity as follows:

$$
\text { Turbidity }=\frac{1}{I_{\text {transm.norm }}}
$$

\section{g) Thermogravimetric Analysis}

The thermal stability of the supramolecular microgels was characterized by TGA on a Simultaneous Thermal Analyzer STA 6000 (Perkin Elmer). The measurements were performed in a temperature interval between $30-600{ }^{\circ} \mathrm{C}$ using a heating rate of $10{ }^{\circ} \mathrm{C}$. $\mathrm{min}^{-1}$. Nitrogen served as purge gas $\left(20 \mathrm{~mL} \cdot \mathrm{min}^{-1}\right)$.

\section{h) Differential Scanning Calorimetry}

The VPTT of the microgels was additionally analyzed by DSC on the device DSC 8500 (Perkin Elmer). Each microgel sample was weighted (5-6.5 mg) and placed in stainless steel crucibles. Subsequently, $50 \mu \mathrm{L}$ of $\mathrm{D}_{2} \mathrm{O}$ were added and the pan was closed. A stainless crucible with $50 \mu \mathrm{L} \mathrm{D} \mathrm{D}_{2} \mathrm{O}$ served as reference. The samples were heated-cooledheated between 10 and $100{ }^{\circ} \mathrm{C}$ ( 3 temperature cycles) with a heating rate of $20^{\circ} \mathrm{C} \cdot \mathrm{min}^{-1}$ and a cooling rate of $80^{\circ} \mathrm{C} \cdot \mathrm{min}^{-1}$. 


\subsubsection{Study of Microgel Stability in Organic Solvents}

Microgel solutions $\left(62.5 \mathrm{mg} \cdot \mathrm{mL}^{-1}\right)$ were prepared in $\mathrm{H}_{2} \mathrm{O}, \mathrm{THF}, \mathrm{CHCl}_{3}$ and $\mathrm{MeOH}$ and stirred overnight at room temperature. The stability of the various synthesized microgels in the organic solvents was investigated by STEM. 


\section{4. pH-Triggered Disassembly of Supramolecular Microgels}

\subsection{Introduction}

As highlighted previously, the development of innovative nanotechnologies is related directly to the design of dynamic and self-responsive systems. Stimuli-sensitive biomaterials that respond precisely to specific triggers bring us closer to the desired simulation of natural processes. Biological organisms are capable to self-regulate instantaneously by environmental changes due to accurate stimuli-response mechanisms between (macro-)molecular building units. Ionic concentration, electric forces, temperature and $\mathrm{pH}$ are significant factors that induce these adaptive responses. Particularly, the pH-effect on cellular functions has been in research focus for biochemical studies since more than 100 years. Søren Sørensen (1868-1939†) and his intensive investigations about precipitation of proteins at different ionic concentrations lead to the first introduction of $\mathrm{pH}$ as a relevant scientific concept.120 In 1909, S. Sørensen defined the negative decimal logarithm of the hydrogen ion activity in a solution as " $\mathrm{pH}$ ". However, this calculation was modified and related to the hydrogen molality $\left(\mathrm{mol} \cdot \mathrm{dm}^{-3}\right)$, since the determination of the activity of a single ion is not possible. ${ }^{121-124}$ From that time until now, there have been countless studies related to this term, the accuracy of its definition and its calculation, since the well-known pH-scale from 0 to 14 represents only simple systems. ${ }^{125}$ Moreover, its relevance in cellular mechanisms has been further analyzed. Protein sensitivity against changes of the environmental $\mathrm{pH}$ has showed high importance in terms of membrane sensibility, molecular recognition, ion and information transport, as well as in enzymatic and cellular activities such as proliferation, migration or apoptosis. ${ }^{126-127}$

In addition, a relationship between the corporal $\mathrm{pH}$ and various diseases like cancer has been proposed in later studies. Non-constant and irregular inter- and extracellular $\mathrm{pH}$ values are some of the consequences by tumor formation. Due to this fact, $\mathrm{pH}$ responsive nanomaterials offer great possibilities to improve therapeutic treatments, their selectivity and efficiency. ${ }^{128-130}$ Especially, self-assembled nanosystems are exceptional candidates due to their cooperative behavior and dynamic adaptability. They can be stimulated by $\mathrm{pH}$-changes to associate and dissociate in a targeted form leading to specific and tunable responses. ${ }^{131}$ Of particular interest is the design of $\mathrm{pH}$ - 
indicators to monitor the biological $\mathrm{pH}$ and interesting advances have been made in this view. ${ }^{126-127}$ For example in 2009 , the synthesis of a novel pH-nanosensor called I-switch was reported. This DNA-based nanomachine can be used to monitored pH changes in living cells by fluorescence resonance energy transfer. ${ }^{132-133}$ Moreover, composites with pH-controlling properties have been developed as interesting materials for medical devices. As an example, Fehér et. al. published in 2015 the synthesis of microgelfunctionalized fibers as a pH-regulating system. ${ }^{134}$ In this work, biodegradable PLA materials with self-regulating $\mathrm{pH}$-degradation behavior were produced by incorporating stimuli-responsive microgels with $\mathrm{pH}$-responsive amino functionalities. In relation to pH-sensitivity, tannic acid materials have also aroused enormous interest due to their pH-dependent supramolecular properties. For instance, the Hammond group reported lately a novel pH-responsive drug delivery system. In this case, tannic acid was used as a non-covalent crosslinker between polymeric micelles for the formation of a multilayered platform. The hydrogen-bonded assembly showed a controlled pH-stability and allowed a targeted release of a drug from the micelles under acidic conditions. ${ }^{112}$ This study among many others highlights the significant ability of TA to form pH-responsive nanomaterials.96, 102, 104, 114 However, despite these and further innumerous developments that have been achieved, there is still the necessity for a deeper understanding of biological pH-regulation in view of biomimicry and targeted molecular treatments. Further investigations are therefore indispensable for the synthesis of bioactive nanomaterials with targeted $\mathrm{pH}$-regulating properties.

\subsection{Results and Discussion}

pH-triggered disassembly and controlled TA-release:

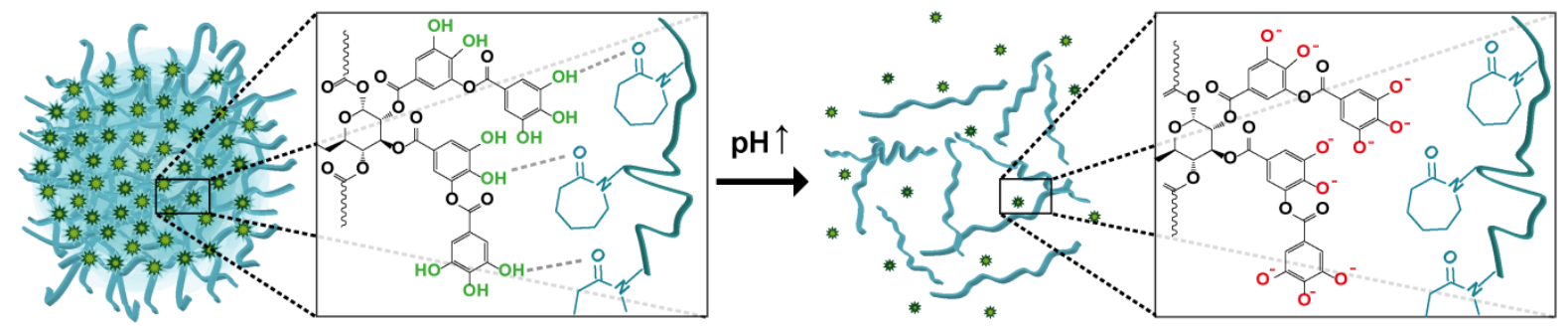

Figure 34. Schematic representation of the pH-induced disintegration of the supramolecular PVCL/TAmicrogels. 
During the characterization of the previous presented supramolecular PVCL/TAmicrogels, the pH-stability was determined (Chapters 2 and 3). We confirmed the stability of the various microgels under acidic conditions and at neutral $\mathrm{pH}$.

However, we reported that an increment of the $\mathrm{pH}$-value to a basic range leads to deprotonation of the phenolic hydroxyl groups of TA, causing the breakdown of the Hbonds and the disintegration of the microgel network (Figure 34). This effect was clearly visualized. At a pH above 8.5, the turbidity of an aqueous microgel solution decreased strongly until it became clear/yellowish, indicating the release of the free tannic acid molecules (Chapter 3, Figure 31). The pH-sensitivity of the TA-based microgels is an essential feature that confirms not only their supramolecular composition, but also proposes an interesting possibility for the design of $\mathrm{pH}$-triggered degradable materials.

Further experiments were thus performed in view of pH-controlled disassembly and simultaneous TA release. Herein, the establishment of a suitable method for the analysis was mandatory. The selection of an proper $\mathrm{pH}$-treatment, separation process, as well as an appropiate characterization method to monitor the TA-release were essential factors for the performance of this study. Due to the absorption properties of tannic acid, the release of this polyphenolic compound was monitored by Ultraviolet-visible (UV/Vis) spectroscopy. Hereby, a calibration at different $\mathrm{pH}$ values was essential, since the chemical structure of tannic acid is pH-dependent and correspondent its UV/Vis absorption (Figure 35a). Therefore, we compared 2 different solutions for the pHtreatment under basic conditions. In the first place, an urea aqueous solution (8 $\mathrm{M}$, $\mathrm{pH}$ 8) was investigated as basic medium for the microgel dissociation, since this substance is well-known in biological studies and of high significance in medical science. On the other hand, an $\mathrm{NaOH}$ aqueous solution $(10 \mathrm{mM}, \mathrm{pH}$ : 11.7) was utilized as comparative base. Two different TA solutions $(7 \mu \mathrm{M})$ were thus prepared in both basic media and analyzed by UV/Vis. 
a.) $\mathrm{pH}$-dependent TA-structure:

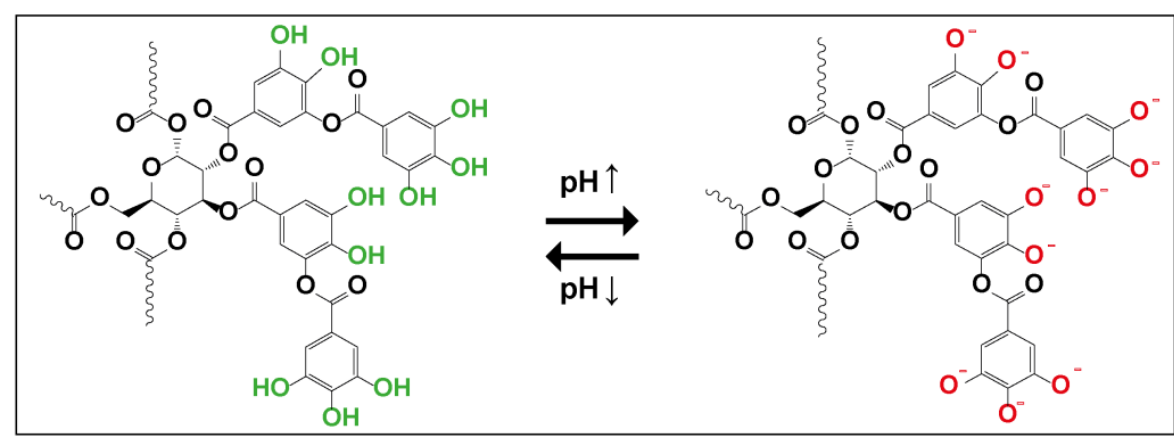

b.) Selection of pH-solution:

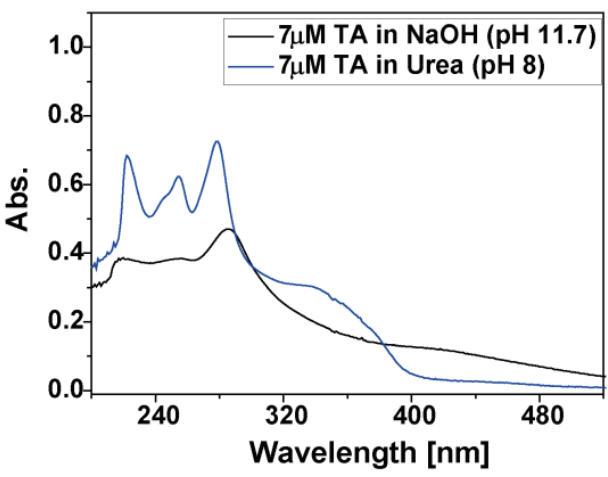

c.) $\mathrm{pH}$-effect on TA-UV/Vis-bands:

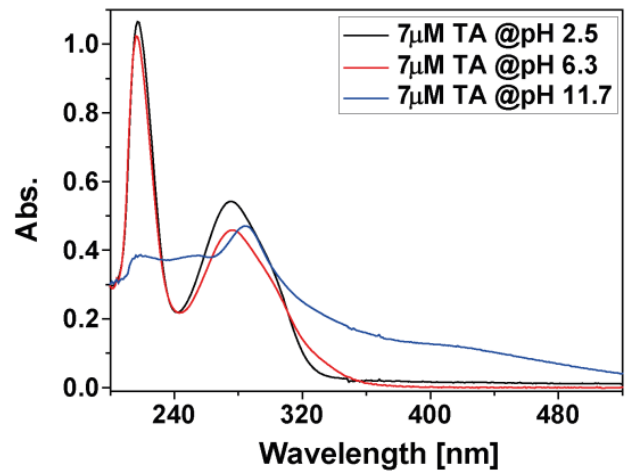

Figure 35. UV/Vis characterization of the TA-structure in dependency of the pH, and selection medium for the pH treatment under basic conditions.

The obtained spectra (Figure 35b) showed the expected signals for the phenolate form of TA. Nevertheless, one can observe 3 different absorption bands in the case of the urea solution (blue curve). Herein, the signals between 260 and $280 \mathrm{~nm}$ suggest two different deprotonated states of tannic acid in the presence of urea. This can be also related to oxidative processes. ${ }^{74}$ Contrary to this effect, the treatment of tannic acid with $\mathrm{NaOH}$ leads to a phenolate structure, represented with a single signal at a wavelength of $285 \mathrm{~nm}$ (black curve). In our study of the pH-stability, we confirmed that a full deprotonation state of TA is achieved at $\mathrm{pH}$ over 8.5. This can explain the existance of two different phenolate structures, if urea (8 M) is used as basic medium, since its $\mathrm{pH}$ is approximately 8. Moreover, in previous publications the stability of urea in aqueous solutions has been reported, describing the instability of its $\mathrm{pH}$ due to decomposition and thermal effects. ${ }^{135-137}$ Based on these facts, $\mathrm{NaOH}(10 \mathrm{mM}, \mathrm{pH}: 11.7)$ was utilized further for the $\mathrm{pH}$ treatment, as well as $\mathrm{HCl}(10 \mathrm{mM}, \mathrm{pH}: 2.5)$ for the $\mathrm{pH}$-adjusment in acidic range. After the selection of the appropiate $\mathrm{pH}$-media, the structure of TA was characterized by UV/Vis spectroscopy of various TA solutions $(7 \mu \mathrm{M})$ at 3 different $\mathrm{pH}$ values: $2.5(\mathrm{HCl}), 6.3\left(\mathrm{H}_{2} \mathrm{O}\right)$ and $11.7(\mathrm{NaOH})$. The obtained results are depicted in Figure 
35c. The UV/Vis curves demonstrate the phenolic structure of tannic acid at pH-values below the neutral range $(\mathrm{pH}<7)$. The absorption band at $275 \mathrm{~nm}$ represents the protonated phenolic rings of TA, which can be observed clearly at a pH of 2.5 and 6.3. However, under basic conditions (pH: 11.7), one notices a decrease of intensity, which indicates the initiation of the deprotonation process. In this case, there is a shift of this characteristic band to $285 \mathrm{~nm}$ due to the ionization of the galloyl units. ${ }^{74}$ Based on this analysis, the calibration curves for TA in dependency of the $\mathrm{pH}$ were created using these significant UV/Vis signals (supplementary results, Figure 41 ).

a.) Centrifuge Set-up:

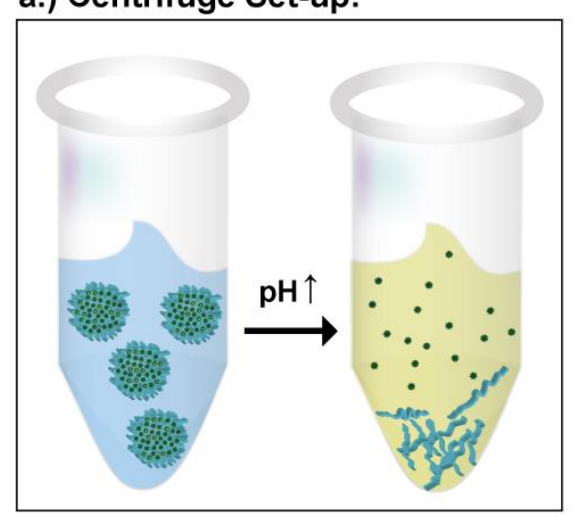

b.) Dialysis Set-up:

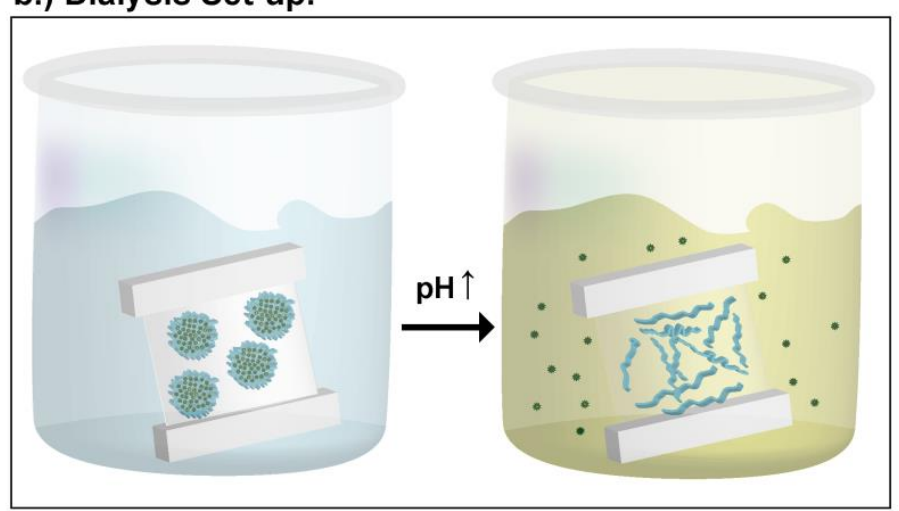

Figure 36. Illustration of various set-ups used for the pH-triggered disassembly experiments.

As mentioned above, the selection of a suitable set-up for the pH-triggered degradation experiments complays an important role in this section. Therefore, centrifugation and dialysis were examined as methods for the separation of the released TA molecules from the PVCL-chains. The set-ups are illustrated in Figure 36.

Selection of separation method:
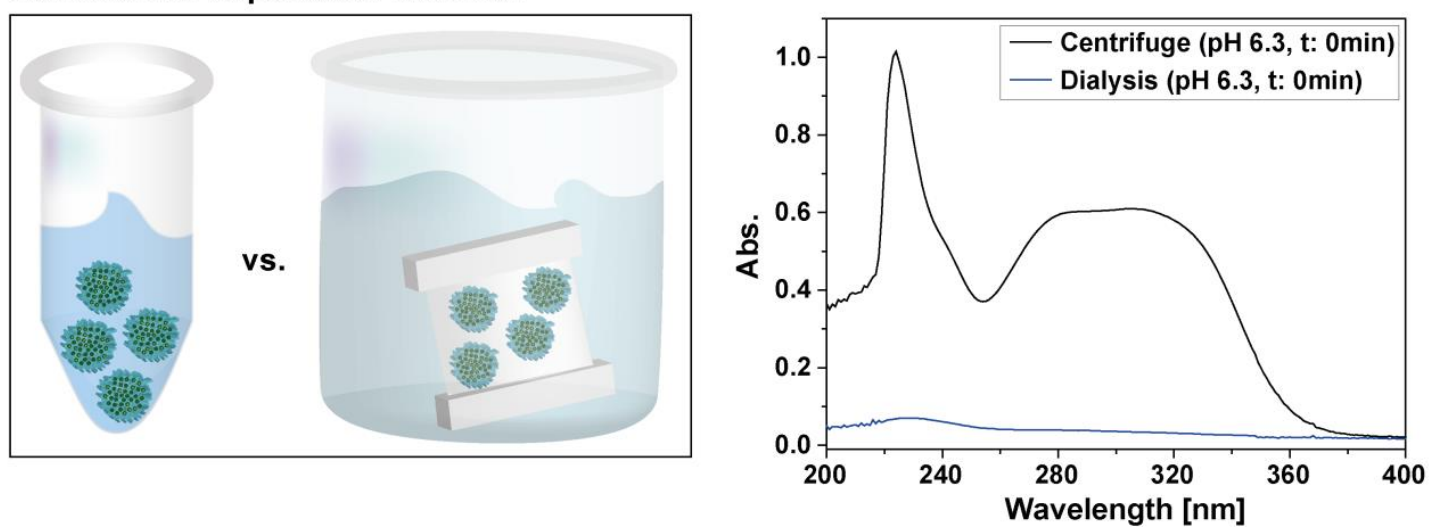

Figure 37. Comparison of the used set-ups in view of separation of the PVCL-chains from TA. 
As a preliminar analysis, PVCL/TA microgel aqueous solutions were prepared at a $\mathrm{pH}$ of 6.3 (des. $\mathrm{H}_{2} \mathrm{O}$ ) and treated separately by each method at the same neutral pH and RT. The supernatant solutions were subsequently measured by UV/Vis (Figure 37). Herein, no absorption bands were expected from any of the experiments, since the supramolecular microgels are highly stable under these conditions. Nonetheless, we conclued that centrifugation is not suitable for our propose. The UV/Vis measurement confirms that there are still microgels in the supernatant and that a $100 \%$ sedimentation either of microgels nor of PVCL chains can be achieved. This would lead to overlapping of the signals during the $\mathrm{pH}$-induced disassembly and would cause fasification of the results by monitoring the released TA-amount. This study lead us to the conclusion that diaylsis was a more appropiate separation process for the disassembly and release study.

a.) Variation of $\mathrm{pH}$ :

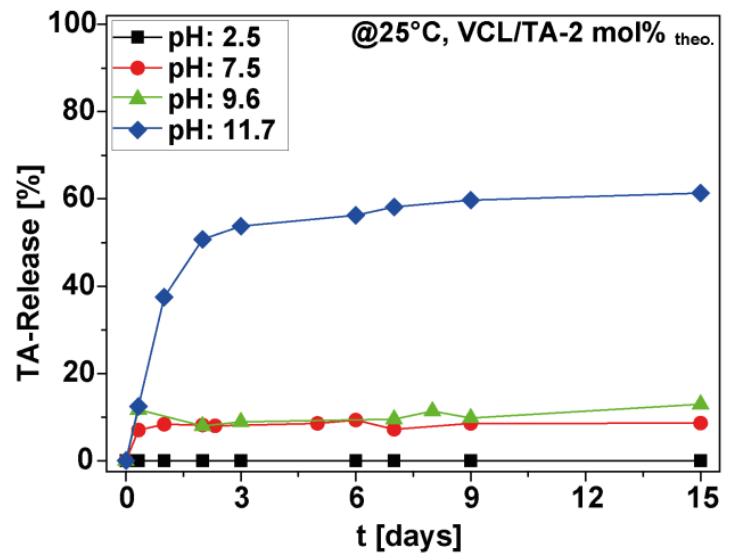

b.) Variation of temperature:

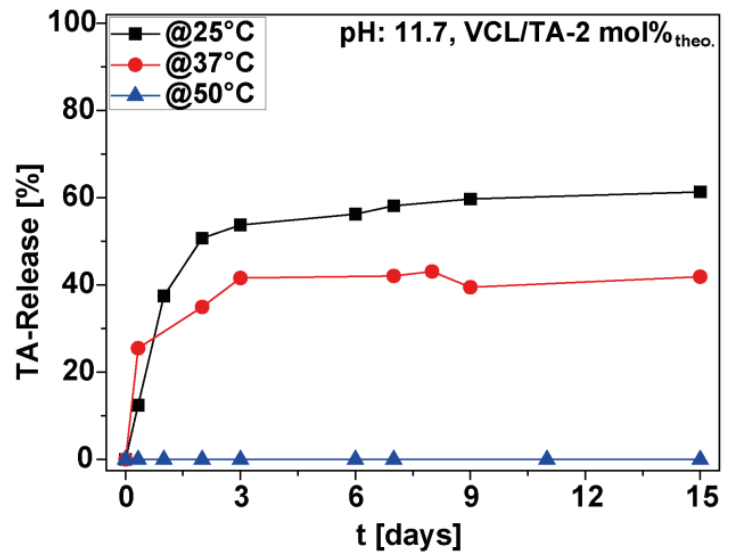

Figure 38. pH-triggered disassembly of VCL/TA-2mol\%theo-microgels (optimized synthesis, TAreal: $1.29 \mathrm{~mol} \%$ ) and simultaneous TA-release.

The determination of the pH-triggered disassembly and the simultaneous TA-release was performed after the set-up establishment and the selection of the parameters. Therefore, dialysis of the VCL/TA-2mol\%theo-microgels (optimized synthesis, TAreal: $1.29 \mathrm{~mol} \%$ ) was carried out at $4 \mathrm{pH}$ values as well as 3 different temperatures over 15 days (Figure 38). Herein, small samples of the dialysis medium were withdrawn at certain time periods and measured by UV/Vis. By means of the obtained spectra, the degree of release of tannic acid could be determined.

Regarding the pH-effect on the microgel stability (Figure 38a), there was neither disintegration of the microgel network nor release of tannic acid under acidic conditions (pH: 2.5). A limited delivery of the polyphenolic compound is exhibited firstly from a $\mathrm{pH}$ 
value of 7.5. After 15 days, a maximal release of $12 \%$ is reached at neutral conditions. Similar results were obtained at pH 9.6, even though this value is already within the basic range. This observation can be explained by means of the protonation/deprotonation process of TA. Although the $\mathrm{pK}_{\mathrm{a}}$ value of the pure polyphenol is 8.5, the interaction with the PVCL chains within the microgel network plays a decisive role. The previous analysis of the $\mathrm{pH}$-stability (Chapter 2, Figure 31) demonstrates that the supramolecular microgel system starts to disintegrate at a $\mathrm{pH}$ of 8.5. Nevertheless, a plateau at a pH of 10.5 can be observed clearly. This state indicates the period needed for a complete deprotonation of TA, meaning that only after a $\mathrm{pH}$ of 10.5 all hydroxyl groups of the polyphenol are deprotonated within the microgel. The UV/Vis spectra of TA at pH 9.6 correlate also to this assumption (supplementary results, Figure 41). As expected, only at a pH of 11.7 a significant triggered release of TA could be monitored. After 15 days, almost $60 \%$ of the TA was released from the microgel network under basic conditions. Moreover, the delivery of tannic acid could be confirmed optically due to color change of the dialysis medium, which turned yellowish by the time (Figure 39).

pH-triggered disassembly of PVCL/TA-microgels:
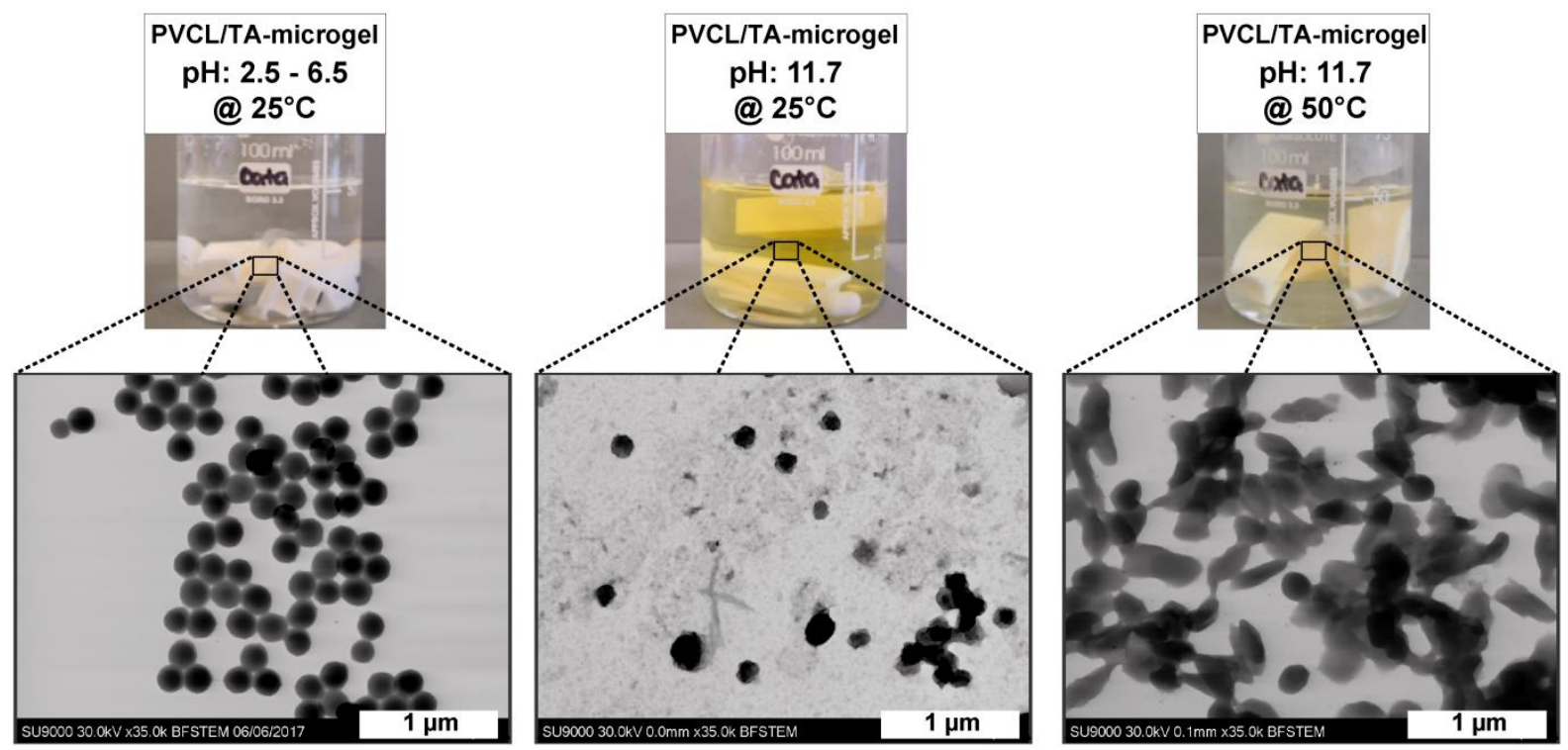

Figure 39. PVCL/TA-microgels after $\mathrm{pH}$-triggered disassembly at different $\mathrm{pH}$ values and temperatures.

Of particular interest was the effect of the temperature during pH-triggered disassembly. We confirmed positively that the disintegration of the microgel network as well as the delivery of TA can be inhibited at temperatures over the VPTT of the 
microgels (Figure 38b). At a temperature of $37^{\circ} \mathrm{C}$, the delivery of TA at $\mathrm{pH} 11.7$ was repressed by $30 \%$ approximately. In the case of the experiment performed at $50{ }^{\circ} \mathrm{C}$ under basic conditions there was no TA release monitored. The results do not show any signals that indicate the release of the polyphenolic compound in a deprotonated state. Moreover, the clearness of the dialysis solution is a visual hint for the repression of the TA release. It can be concluded that the increase of the polymer-to-polymer interactions of the microgels at the collapsed state leads to a stabilizing effect of the TA molecules within the polymer network and prevents their deprotonation and delivery. The microscopic analysis of the microgel solution confirmed this hypothesis (Figure 39). PVCL/TA-microgels in a basic aqueous medium ( $\mathrm{pH}$ 11.7) show no stability at room temperature $\left(@ 25^{\circ} \mathrm{C}\right)$. The STEM image (middle) indicates the disassembly of the microgel network at these conditions once more. Irregular polymeric residuals after the disassembly process can be observed, possibily as a result of further crosslinks introduced by the interaction of partially protonated TA with the PVCL chains. However, the right image (PVCL/TA-microgels, $\mathrm{pH} 11.7$, @50 ${ }^{\circ} \mathrm{C}$ ) confirms the stability of the supramolecular colloids under basic conditions and high temperatures. Herein, asymmetric colloidal gels are observed. The microgels show a deformed and compact morphology which can be caused due to instabilities under basic conditions and their thermoresponsiveness.

a.) Variation of microgel type:

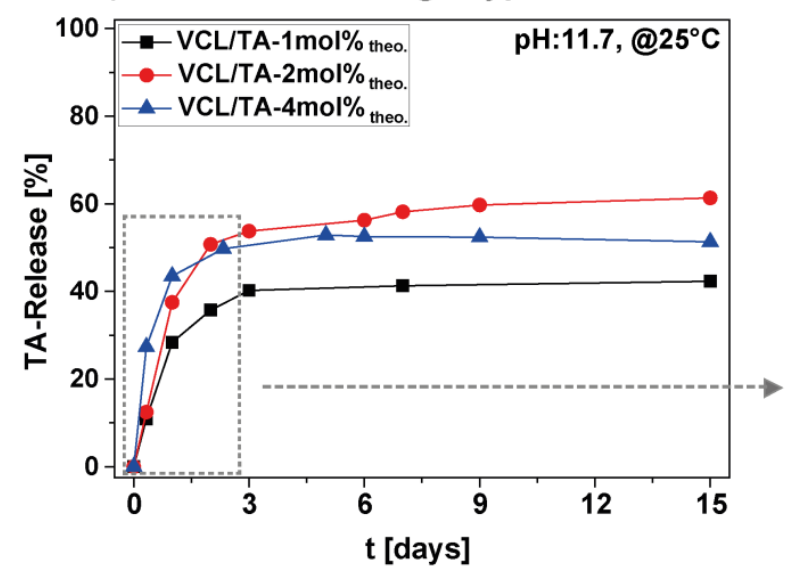

b.) Disassembly slope:

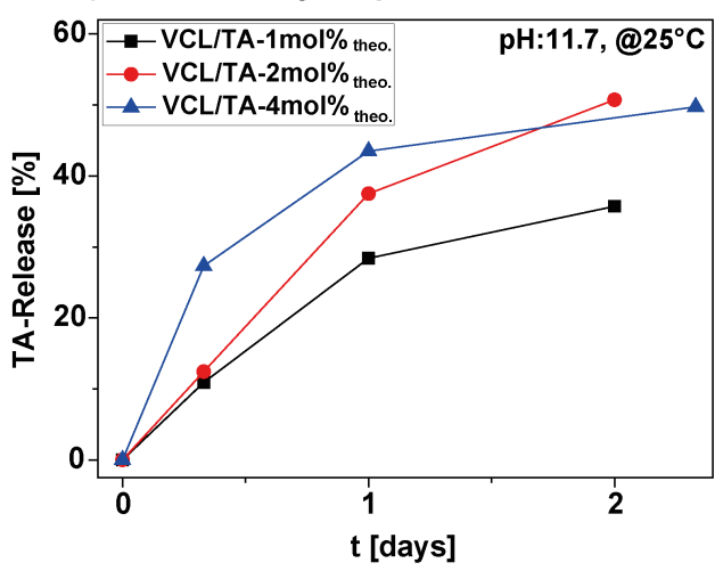

Figure 40. pH-triggered disassembly of supramolecular microgels with different TA-content (TAreal: $0.83 \mathrm{~mol} \%, 1.29 \mathrm{~mol} \%$ and $1.68 \mathrm{~mol} \%$ ).

Finally, the pH-triggered disassembly at a pH of 11.7 and over 15 days was performed to various PVCL/TA-microgels with different TA-amount (TA real $_{0} 0.83 \mathrm{~mol} \%, 1.29 \mathrm{~mol} \%$ 
and $1.68 \mathrm{~mol} \%)$. Figure 40 depicts the results. The analyzed data correspond to the expectations that the higher the amount of TA within the microgel, the faster and higher the release of the natural polyphenol. Still, this observation applies only for the period up to the third day. The sample VCL/TA-4mol\%theo. with the higher amount of the physical crosslinker does not exhibit the highest TA-release after 15 days. Nevertheless, the examination of the time frame between 0 and 3 days and the qualitative analysis of the curve slope corroborates that higher contents of TA lead to a more rapid release (Figure 40b). The increment of the molar fraction of TA within the PVCL-network also shows an increase of the delivered percentage within the first 3 days. The unforeseen results for the time period between 3 and 15 days for the samples VCL/TA-2mol\%theo. and VCL/TA-4mol\%theo. could be explained due to the various parameters that influence the set-up and characterization method. Herein, it is necessary to remark that these studies are preliminary. There are many factors that have to be considered because of their influence on the results: Firstly, the quantification of the real TA-amount contained within the microgels was performed by Raman spectroscopy. This calculation relies also on a calibration and therefore it can be seen as an estimated value. This can thus have an effect on the calculated degree of release. Moreover, the dialysis membrane used plays an important role. Depending on the pore size, the diffusion of free PVCL-chains can take place, hindering a 100\% separation of both components. This assumption was confirmed by a comparison of the measured UV/Vis spectra with the absorption spectrum of pure PVCL (supplementary results, Figure 42a). One can observe that the absorption band at a wavelength of $220 \mathrm{~nm}$ obtained during the disassembly experiment corresponds to the representing signal of the polymer chains. In addition, the diffusion rate is to be accounted. The smaller the membrane pores, the slower the molecule diffusion and the TA-release becomes. The utilization of a membrane with small pore size (e.g. $\sim 3.5 \mathrm{kDa}$ ) leads to a delay of the TA-delivery and a falsified slow release rate (Figure $42 \mathrm{~b}$ ). Therefore, the utilization of a membrane with larger pores (12-14 kDa) was preferred, since the signals of PVCL and TA do not overlap. Finally, it is important to point out that it is possible that some TA-molecules remain inside the membrane interacting with the relaxed polymer chains or even with the dialysis membrane itself. This would explain the maximum release degree of $60 \%$. Nonetheless and despite these observations, these results strongly indicate not only the successful 
$\mathrm{pH}$-induced disassembly of our microgels, but also their supramolecular conception and its controlled targeted properties. In conclusion, the performed study is a great basis for future investigations and optimizations.

\subsection{Conclusions}

pH-regulating systems have caused high impact lately in biomedical studies, since the effect of biological pH on cellular functions and enzymatic activities has been reported. In view of biomimicry and targeted molecular treatments, the design of $\mathrm{pH}$-sensitive materials with tunable and adaptive properties is crucial. Therefore, one of the goals of this thesis was to obtain a supramolecular material that with controllable $\mathrm{pH}$-induced properties. In this chapter, the pH-sensitivity of the supramolecular PVCL/TA-microgels was studied in detail to obtain further information regarding the control of their $\mathrm{pH}$ responsiveness and their usage as TA-delivery systems. The establishment of the analysis method was decisive for the experiments. The pH-triggered disassembly and simultaneous TA-release was monitored by UV/Vis measurements. Therefore, a dialysis approach was selected for the separation of the released TA molecules from the PVCL chains. Due to the fact that TA has a pH-dependent structure, calibrations at different pH-values were carried out. The disassembly experiments were performed in dependency of the $\mathrm{pH}$ and temperature. Herein, the preliminary results suggest that our supramolecular microgels can be used as TA-release systems and that the delivery of the phenolic compound can be controlled by pH-stimuli. Under acidic or neutral conditions, the microgels did not show TA-release. Contrary to this, at a $\mathrm{pH}$ of 11.7 a release of almost $60 \%$ was achieved over 15 days. Moreover, we confirmed successfully that the pH-induced disassembly can be inhibited due to the thermoresponsiveness of the microgels. At temperatures above the VPTT of our materials, the TA molecules cannot leave the collapsed polymer network. In conclusion, these prior studies offer an interesting basis for further improvements in view of design of adaptive and responsive nanomaterials. 


\subsection{Supplementary Information}

a.) UV/Vis calibration @pH 2.5:

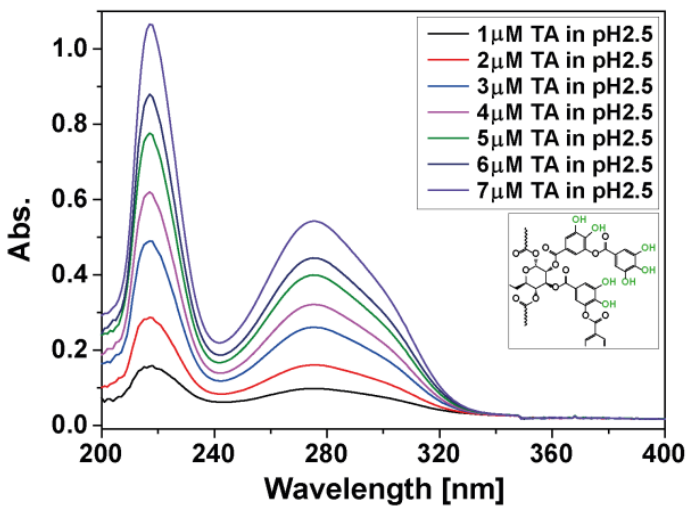

b.) UV/Vis calibration @pH 7.5:

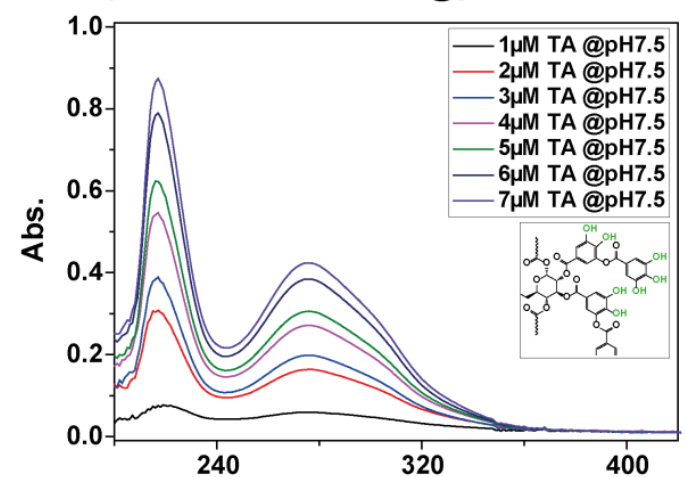

c.) UV/Vis calibration @pH 9.6:

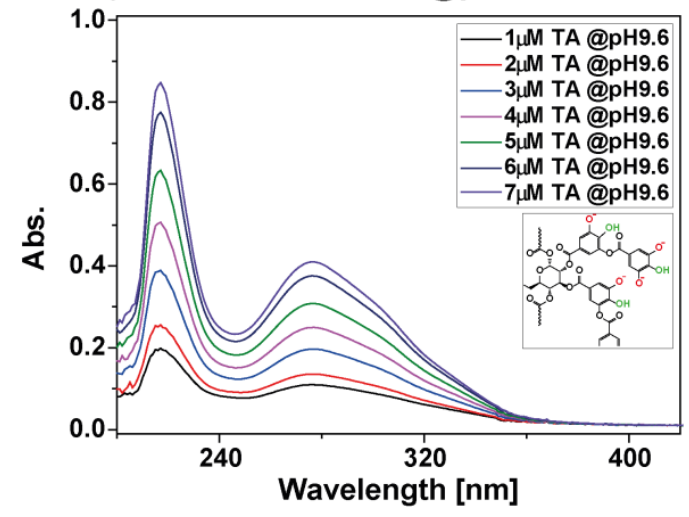

d.) UV/Vis calibration @pH 11.7:

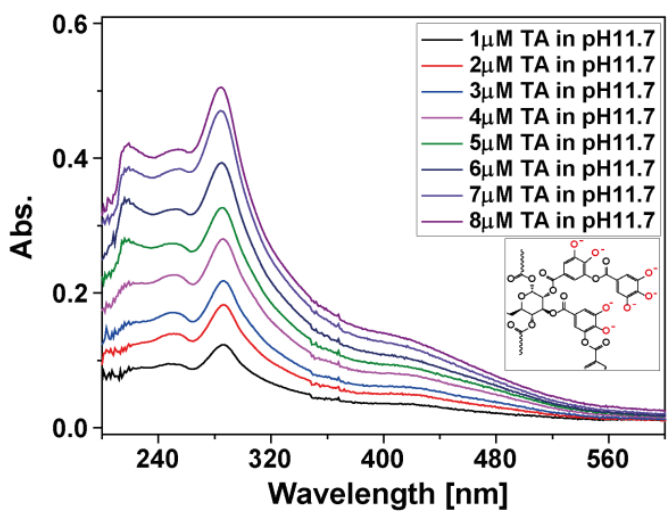

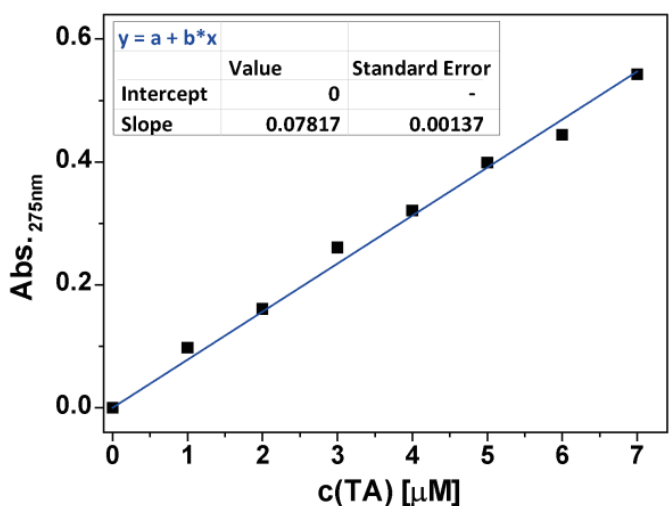
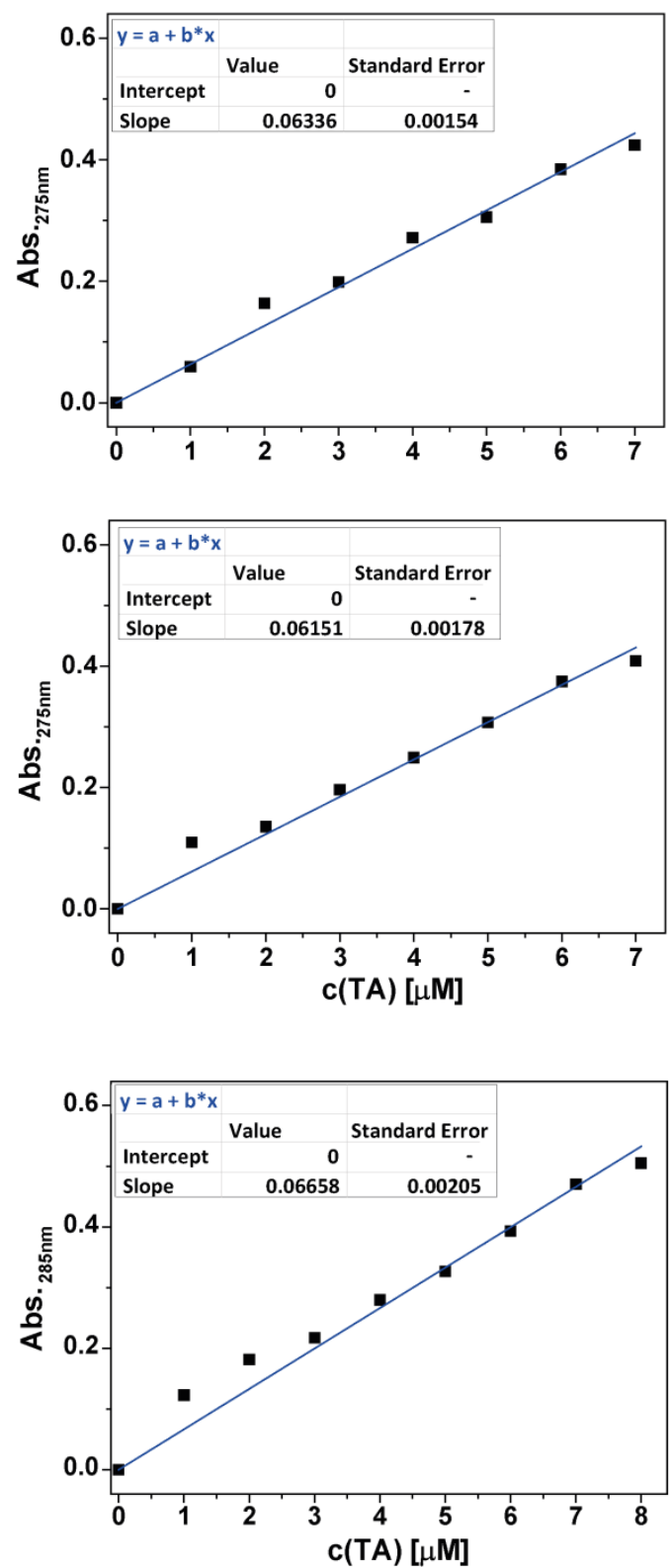

Figure 41. Calibration by UV/Vis of the TA-concentration at different $\mathrm{pH}$ values. 
a.) PVCL and TA @pH11.7:

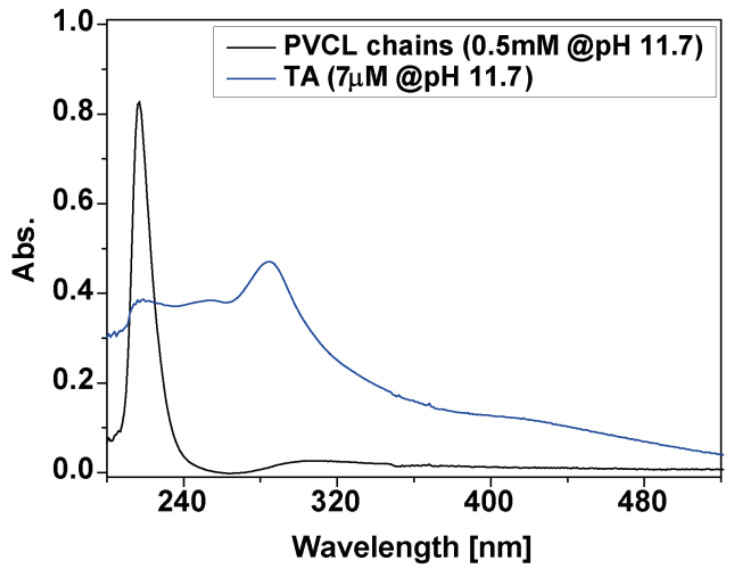

b.) Dialysis membrane:

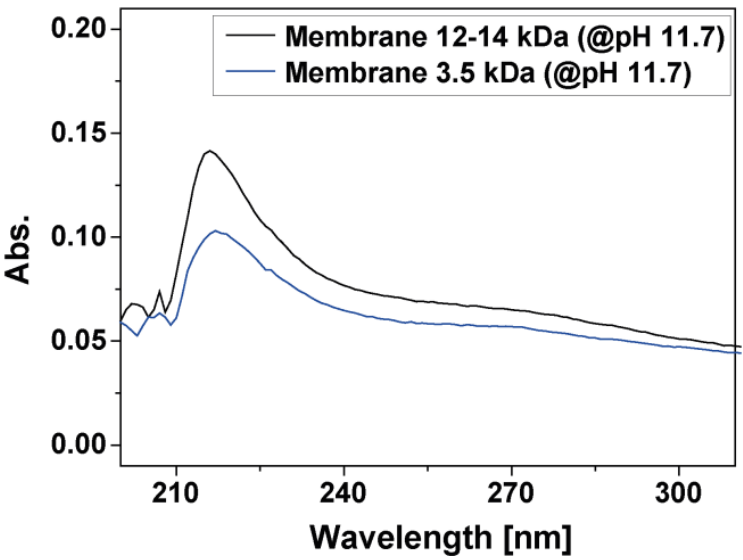

Figure 42. . Comparison of a) UV/Vis spectra of PVCL and TA at pH 11.7, as well as b) dialysis membranes with different pore sizes

a.) pH-disassembly @25ำ \& pH2.5:

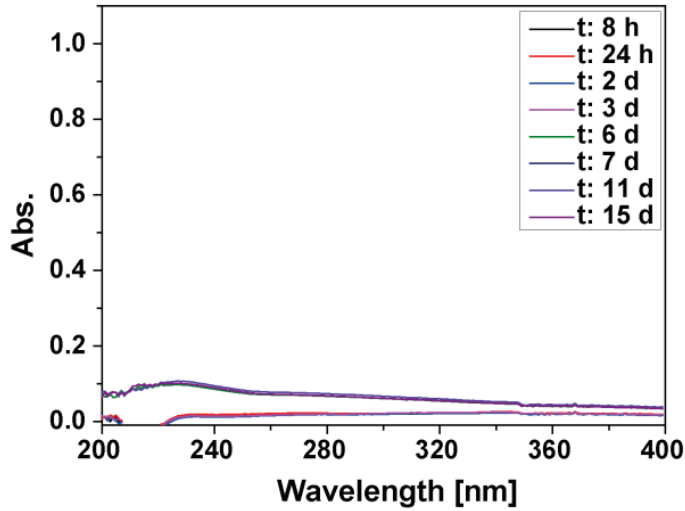

c.) pH-disassembly @25ㄷ \& pH9.6:

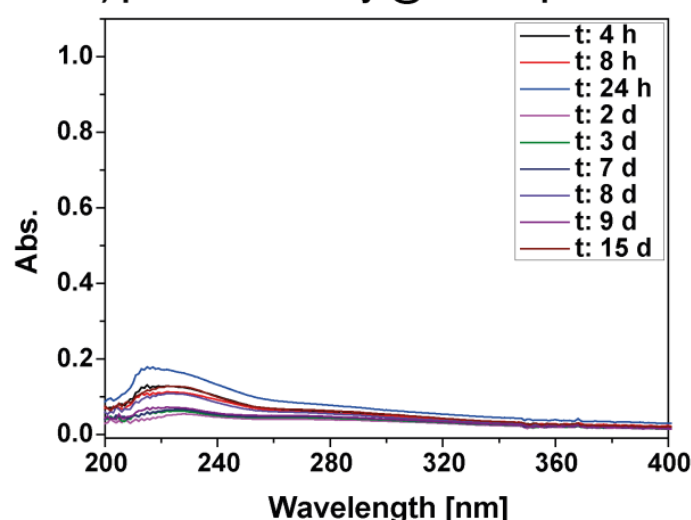

b.) pH-disassembly @25ㄷ $\&$ pH7.5:

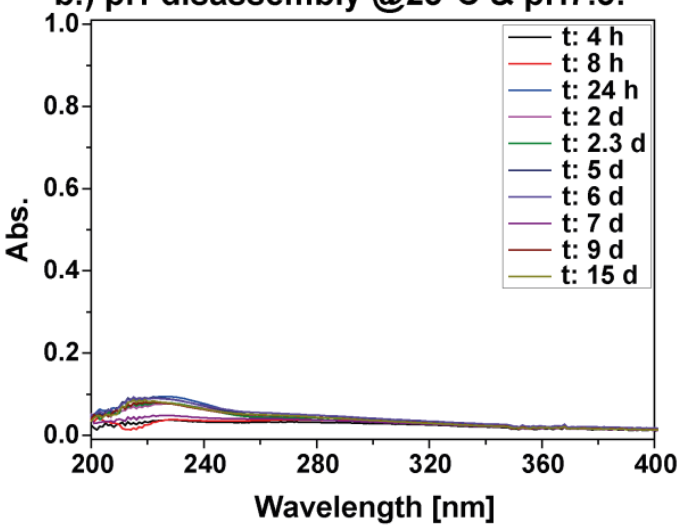

d.) pH-disassembly @25ㅇ \& pH11.7:

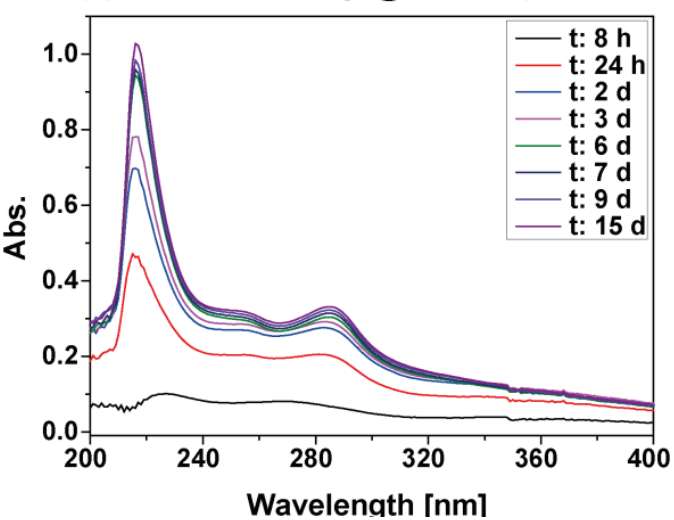

Figure 43. . UV/Vis spectra of pH-triggered disassembly experiments carried out at different pH values. 
a.) $\mathrm{pH}$-disassembly VCL/TA-1mol\%theo:

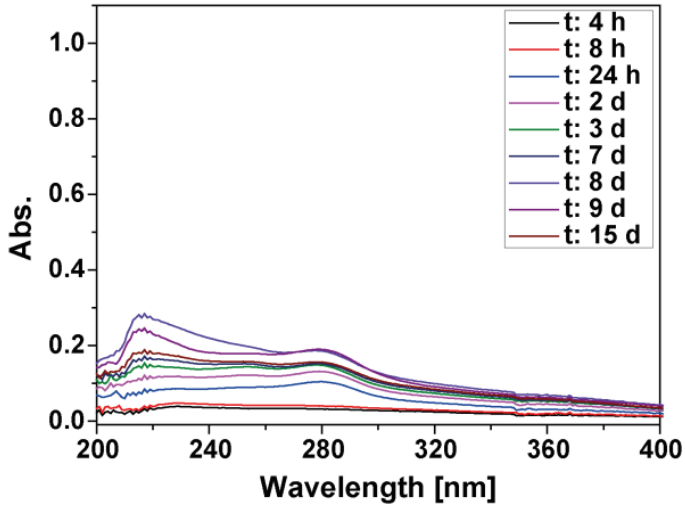

c.) pH-disassembly @37 $\mathrm{C} \& \mathrm{pH} 11.7$ :

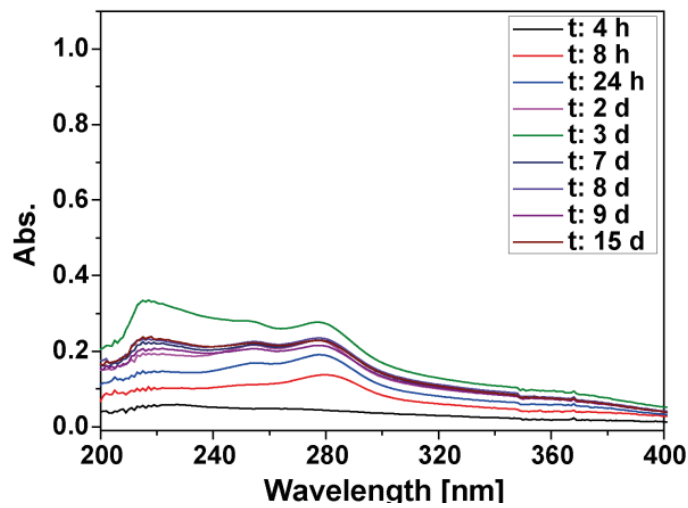

b.) $\mathrm{pH}$-disassembly VCL/TA-4mol\%theo:

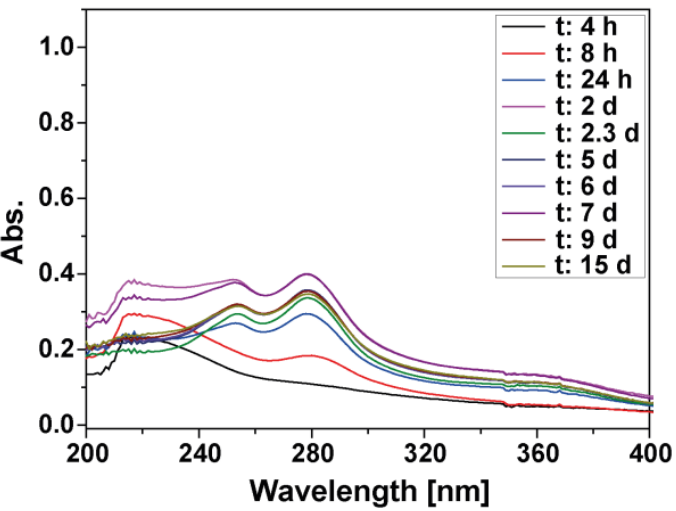

d.) pH-disassembly @50 $\mathrm{C} \& \mathrm{pH} 11.7$ :

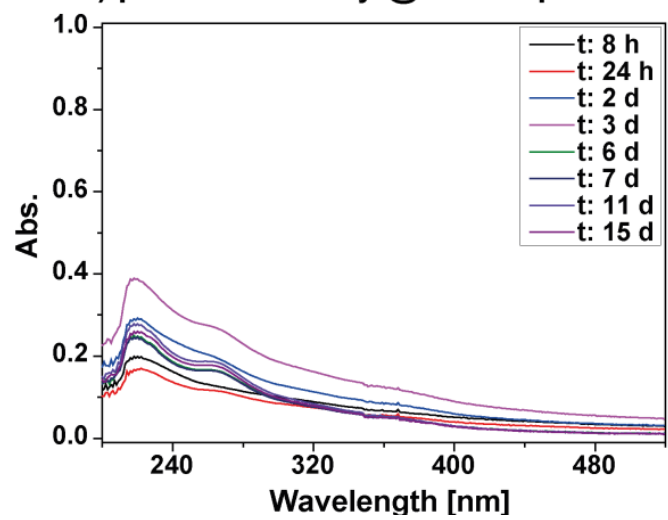

Figure 44. UV/Vis spectra of pH-triggered disassembly experiments performed on PVCL/TAmicrogels with various TA-molar fractions. 


\subsection{Experimental Section}

\subsubsection{Materials}

Tannic acid (TA, Sigma-Aldrich), sodium hydroxide solution (0.1 N, Sigma-Aldrich), $\mathrm{NaOH}$ (ACS reagent, $\geq 97.0 \%$, pellets), $\mathrm{HCl}$ (37\%, VWR), Urea (Merck, powder, reagent grade, 98\%), and dialysis membranes (Zellu Trans/Roth MWCO 3500 and 12000-14000, Carl Roth) were used without further treatment. Deionized water was used for all experiments. Linear PVCL was synthesized via RAFT polymerization by Chaolei $\mathrm{Hu}$ (Research group of Prof. Andrij Pich, DWI-Leibniz-Institute for Interactive Materials). The polymers were purified by dialysis.

\subsection{2. pH-Disassembly of the PVCL/TA-Microgels}

\section{a) pH-triggered Disassembly by Centrifugation}

Disassembly experiments were carried out in Eppendorf-tubes (1.5 mL total volume) using centrifugation as separation method. Therefore, dried microgels $\left(6 \mathrm{mg}, \mathrm{TA}_{\text {real: }}\right.$ $1.29 \mathrm{~mol} \%$ ) were dissolved in $600 \mu \mathrm{L}$ of $\mathrm{H}_{2} \mathrm{O}(\mathrm{pH}: 11.7)$ or an urea solution (8 M), and shaken for certain period of time. After the pH-treatment, the supernatant was separated by centrifugation (1 min, $12000 \mathrm{rpm}$ ) for further characterization.

\section{b) pH-triggered Disassembly by Dialysis}

The disassembly experiments were carried out by dialysis using different membranes (MWCO 3500 and 12000-14000). Thus, $2 \mathrm{~mL}$ of an aqueous microgel solution (20 mg.mL $\mathrm{mL}^{-1}$, TA real: $1.29 \mathrm{~mol} \%$ ) were dialyzed in different $\mathrm{pH}$ media (pH: 2.5 and 11.7) and at different temperatures $\left(25^{\circ} \mathrm{C}\right.$ or $\left.50^{\circ} \mathrm{C}\right) .100 \mu \mathrm{L}$ samples were withdrawn from the dialysis medium after certain time periods during the experiment for further characterization.

\section{c) pH-triggered TA-Release}

The release of tannic acid was monitored by UV/Vis spectroscopy on a Varian Cary 100 Bio UV/Vis Spectrophotometer and a Jasco V-780 VV Visible Spectrophotometer $(\lambda=$ $200 \mathrm{~nm}$ and $\lambda=800 \mathrm{~nm}$ ). Therefore, calibration at different $\mathrm{pH}$ conditions were performed (pH: 2.5, 6.3, 7.5, 9.6 and 11.7) using $\mathrm{HCl}$ and $\mathrm{NaOH}$ ( $10 \mathrm{mM}$ each) for the $\mathrm{pH}$ 
adjustment. Herein, aqueous samples of TA $(2 \mathrm{~mL})$ were prepared at different $\mathrm{pH}$ values. Therefore, TA (1 mM) and 4 pH-solutions $(2.5,7.5,9.6$ and 11.7$)$ were used. The volume of each is listed in Table 9.

Table 9. Volumes used for UV/Vis-calibration of TA at different pH-values.

\begin{tabular}{|c|c|c|}
\hline Sample & $\mathbf{V}_{\text {TA_1mM }[\boldsymbol{\mu L}]}$ & $\mathbf{V}_{\text {pH-solution }[\boldsymbol{\mu L}]}$ \\
\hline Cal_pHDeg_X(pH)_1 $\boldsymbol{M M}$ & 2 & 1998 \\
\hline Cal_pHDeg_X(pH)_2 $\boldsymbol{\mu M}$ & 4 & 1996 \\
\hline Cal_pHDeg_X(pH)_3 $\boldsymbol{\mu M}$ & 6 & 1994 \\
\hline Cal_pHDeg_X(pH)_4 $\boldsymbol{\mu M}$ & 8 & 1992 \\
\hline Cal_pHDeg_X(pH)_5 $\boldsymbol{\mu M}$ & 10 & 1990 \\
\hline Cal_pHDeg_X(pH)_6 $\boldsymbol{\mu M}$ & 12 & 1988 \\
\hline Cal_pHDeg_X(pH)_7 $\boldsymbol{\mu M}$ & 14 & 1986 \\
\hline
\end{tabular}

\section{c) Scanning Transmission Electron Microscopy}

The morphology of the microgels was studied by SEM and TEM on an Ultra-high Resolution Scanning Electron Microscope SU9000 (Hitachi-High Technologies America, Inc.). $100 \mu \mathrm{L}$ of each microgel solution $\left(0.1 \mathrm{~g} \cdot \mathrm{L}^{-1}\right)$ were diluted in $1 \mathrm{~mL}$ deionized water. $20 \mu \mathrm{L}$ of it were dropped on a TEM-grid (Carbon Film 200 Mesh Copper Grids, Electron Microscopy Sciences) and dried at room temperature overnight. The samples were sputtered with $2 \mathrm{~nm}$ carbon before the measurements.

This work was performed by Sabrina Mallmann in part at the Center for Chemical Polymer Technology CPT, which is supported by the EU and the federal state of North Rhine-Westphalia (grant no. EFRE 3000883 02). 


\section{Supramolecular Microgels as Carriers for Active Compounds}

\subsection{Introduction}

Throughout the last decades, high impact discoveries have been made in medicine. The successful synthesis of vaccines as well as novel multidrug therapies have led to the total eradication of diseases like smallpox and leprosy, which were the cause of countless deaths for centuries. ${ }^{138}$ Nevertheless, the simultaneous evolution of new types of infections like Chikungunya or Zika, as well as the complexity of diseases like cancer, imposes the necessity of deeper-going scientific studies and the design of selective clinical treatments to achieve permanent protection. ${ }^{138-140}$ In pharmaceutics, a high efficacy of bioactive compounds depends not only on their activity but also on their efficient body uptake. Poor solubility, lack of stability under biological conditions and undesired secondary effects are challenges that must be overcome. ${ }^{141}$

In terms of pharmaceutical improvement, the synthesis of stimuli-responsive and dynamic nanomaterials as drug vehicles can offer great advantages to achieve targeted treatments of high selectivity and efficacy, reducing the need of regular medications, high and unspecific dosages, and improving the preservation of bioactive compounds. ${ }^{25}$, 139 In general, the most common techniques for drug application are performed via oral or intravenous methods. However, these processes are size dependent because they rely on diffusion of the active molecules trough the cellular membrane. If the required bioactive compounds are macromolecules or more complex nanoparticles, their diffusion is highly limited so that their uptake can occur only via endocytosis. ${ }^{141}$ Due to this fact, the design of biocompatible carriers at the nanometer scale to avoid cellular rejection is essential. The utilization of polymeric transporters leads to important advances such as protection against early drug degradation and undesired interactions, higher uptake-degree and controlled distribution profile. ${ }^{142}$ Nonetheless, it also implies the synthesis of materials with adaptive and dynamic properties, which can respond to the organism signals accurately. Liposomes, block-copolymers, micelles, polymeric nanoparticles, dendrimers and hydrogels are some relevant examples which have aroused enormous interest as "smart" drug delivery systems. ${ }^{141-143}$ Especially, pHresponsive polymer-based materials are promising candidates, since in many different biological mechanisms, changes of the physiological $\mathrm{pH}$ are responsible for different 
cellular effects, in particular in the case of diseased tissues ${ }^{141,144-145}$ So far, innumerous interesting studies have been carried out in view of targeted drug delivery. ${ }^{139-140,146}$ In 2002 for example, the research group of A. Eisenberg published the successful synthesis of polymeric micelles as promising drug transport systems. The materials were based on a poly(ethylene oxide)-b-poly( $\varepsilon$-caprolactone) (PEO-b-PCL)-block copolymer and fluorescently labeled with rhodamine for visualization of its cellular uptake. This study demonstrated successfully the internalization of the micelles into cells by endocytosis. This work proposed thus an interesting alternative to study and follow the incorporation of polymeric particles into bioorganisms at the subcellular level. 141, 143 Furthermore, Kataoka et. al. proposed a novel supramolecular drug delivery system for the controlled release of Adriamycin (anticancer therapy compound). The selfassembled block-copolymer micelles delivered the active drug as a pH-response under acidic conditions, and in in-vivo tests, the polymeric system showed a significant improvement of the growth inhibition against cancer cells. ${ }^{147}$ Other scientific works have also used dendritic core-shell micelles with acid-responsive features for effective release of active substances in tumor treatments. ${ }^{145}$ Also, high impact publications have reported the usage of peptide-based nanocarriers for targeted gene delivery, as well as polymeric materials for short interfering RNA (siRNA) encapsulation. ${ }^{148-149}$ On the other hand, Steinhilber et. al. developed in 2013 a microgel-based construction for the encapsulation and controlled release of living cells. The $\mathrm{pH}$-cleavable microgels were produced using different types of biocompatible hydrogel precursors. The synthesis and simultaneous incorporation of living cells was performed by microfluidics technique. These experiments showed long-term preservation of the cell functions and high cell survivability. Also a controlled release of the cells by $\mathrm{pH}$-induction was achieved successfully, offering a promising alternative to isolate cells temporary for analytical proposes and complex treatments. ${ }^{150}$ Among many others, these examples highlight the benefits of nanomaterial design against medical issues, and the importance for the continuous research in this field.

During the synthesis of drug vehicles, the required bioactive compounds depending on the disease are evidently a decisive factor. Until now, the number of known pharmaceuticals is endless, as well as the variety of promising natural and synthetic bioactive components for medical treatments. Among this long list, nanostructures 
based on metals such as iron, gold or zinc have been a focal point of research for biomedical applications by reason of their multiple properties and high stability. ${ }^{151-152}$ By means of biological significance, iron is one of the most important elements. It can be found in minerals in the oxide and sulfide form, and also as salts in water springs. ${ }^{153}$ However, these sources are not always suitable for its biological usage. Bioorganisms have thus developed different mechanisms to uptake iron and regulate its concentration, since it is a required cofactor in many enzymatic processes. ${ }^{154-155}$ One of its most important functions in nature is oxygen transport. Herein, the redox properties of iron are responsible for the binding and release of oxygen. ${ }^{156}$ Moreover, iron also plays an important role in the DNA metabolism and repair, as well as energy storage. ${ }^{155,157-158}$ Iron insufficiency can therefore cause complex diseases such as anemia, as well as allergic responses and can affect the proliferation of certain types of cells. ${ }^{157-158}$ Thus, the design of specific iron-carriers and regulating systems is essential in medicine as well as in biotechnology. Iron is an important micronutrient in agriculture and therefore, its targeted delivery can improve the plant growth and health. As a particular example, pH-responsive microgels were synthesized by Meurer et. al. in 2017, and proposed as biocompatible fertilizer delivery systems for the transport and delivery of Fe-ions. ${ }^{159}$ Microgels offer a promising alternative in agricultural science for the improvements of plant treatments, since these colloids can be designed with high biocompatibility and water uptake capacity, as well as controlled multifunctionality.

Besides metal ions, phenolic molecules such as catechin, gallic acid and alkyl gallates have been studied intensively in the field of biotechnology as bioactive compounds. Their antibacterial, anticancer, and antioxidant properties have been successfully demonstrated so far. ${ }^{160-163}$ In particular, catechol derivatives and catechin-based composites have been used as biocompatible substances for antioxidative and antiinflammatory applications in cancer treatments. ${ }^{164-166}$ Moreover, n-decyl gallate (G10) has been a focal point in agricultural investigations, due to the fact that it has showed strong antibacterial properties against the plant-pathogenic bacterium Xanthomonas citri (X. Citri). 167-169 These bacteria cause citrus canker, a plant disease that leads to defoliation, dieback and early fruit drop. ${ }^{170-171}$ Currently, X. Citri has spread broadly in Brazil, affecting the orange cultivation strongly. The production is therefore intensely damaged, and requires ecological and thus "green" solutions that imply plant protection 
by coating, the usage of biocompatible materials, and a controlled release of antibacterial compounds. ${ }^{169}$ The achievement of such protective approaches would finally lead to the reduction of pesticides that may affect human health and the ecosystem.

Based on this theoretical background, the prior reported supramolecular PVCL/TAmicrogels were studied as promising pH-responsive carriers for the transport of iron ions, as well as for the encapsulation of further phenolic derivatives.

\subsection{Results and Discussion}

\subsubsection{Transport-System for $\mathrm{Fe}^{3+-}$-Ions}

a.) Loading of supramolecular microgels with metal-ions:
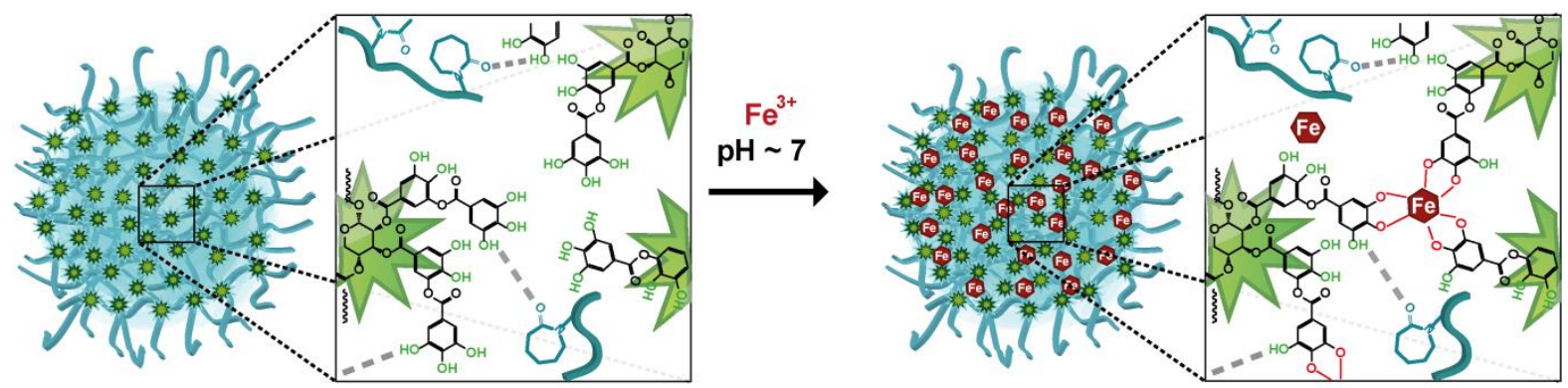

b.) $\mathrm{pH}$-controlled relase of Fe-ions:
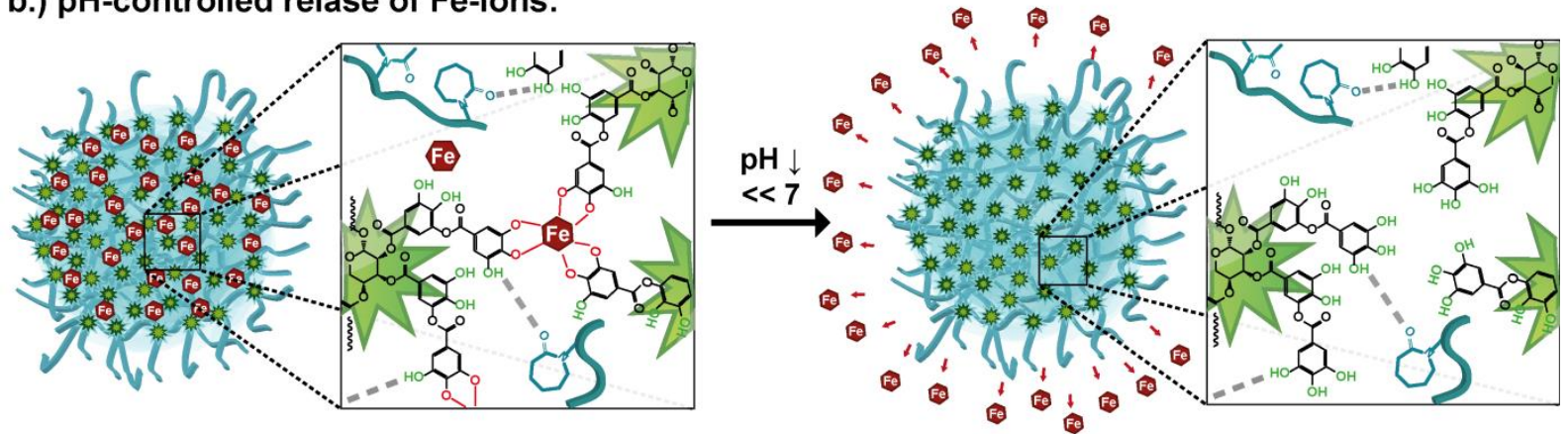

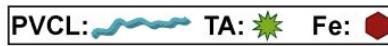

Figure 45. Supramolecular microgels as $\mathrm{pH}$-sensitive $\mathrm{Fe}^{3+}$-carriers.

The pH-sensitivity of TA-based materials and their complexation properties open a wide range of interesting applications. In view of medical treatments, tannic acid has been analyzed not only as a building block component but also as an active agent due to its antibacterial effect. In 2016, Ninan and coworkers reported the versatility of this natural polyphenol.85 In this particular case, TA was used for the crosslinking of bio-based hydrogels, as well as a bioactive compound. The hydrogels were synthesized by ionic 
crosslinking between agarose, TA and Zn-ions and served as pH-sensitive delivery systems for TA-release, leading to antibacterial and anti-inflammatory properties. Other research groups have also reported the successful interactions between TA and metals, especially iron (III) ions. ${ }^{80-81}$ Based on this background and the demonstrated $\mathrm{pH}$ sensitivity of the optimized PVCL/TA-microgels, these materials were proposed as potential $\mathrm{pH}$-responsive vehicles for the transport of $\mathrm{Fe}^{3+}$. Due to the $\mathrm{pH}$-regulated balance between the phenolic/phenolate state of TA, a controlled $\mathrm{Fe}^{3+}$-loading at neutral $\mathrm{pH}$, as well as a targeted release of the metal ions under acidic conditions was expected (Figure 45). At a pH of around 7, the partial deprotonation of the hydroxyl-groups in the tannic acid structure allows the complexation of the iron ions. However, the decrease of $\mathrm{pH}$ leads to a protonated state, forcing the complexed $\mathrm{Fe}^{3+}$-ions to leave the microgel network.

a.) Fe-loading of PVCL/TA-microgels:

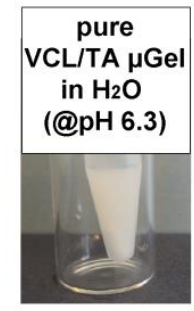

b.) Fe-loading of covalent microgels:

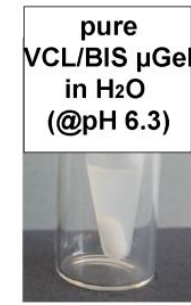

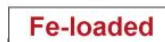

$\mathrm{pH} \sim$
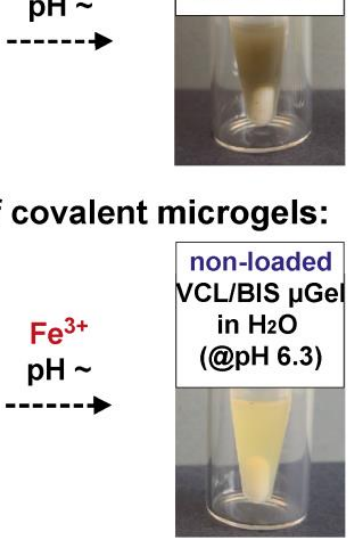

c.) loaded Fe-mass vs. TA-content:

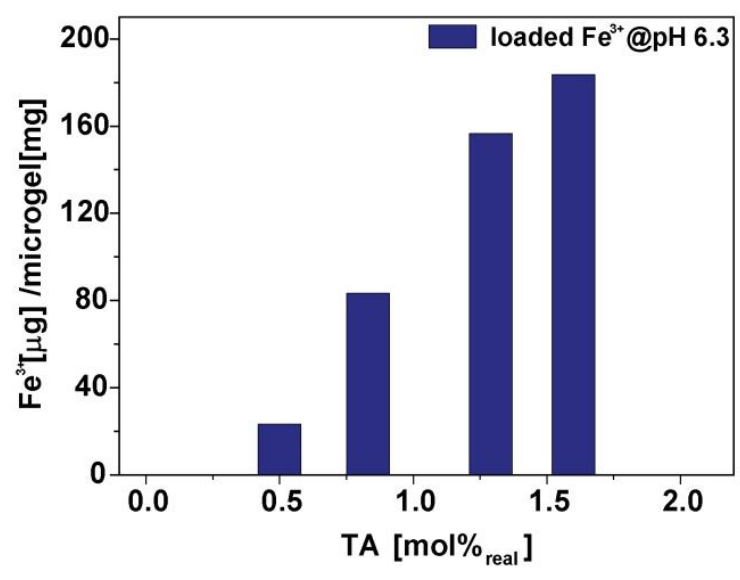

Figure 46. Incorporation of $\mathrm{Fe}^{3+}$ within the PVCL/TA-microgels.

In this work, $\mathrm{Fe}^{3+}$ was incorporated within the PVCL/TA-microgels after the completed microgel synthesis. Therefore, $\mathrm{FeCl}_{3} \cdot 6 \mathrm{H}_{2} \mathrm{O}$ was used as metal precursor and the metal complexation experiments were carried out overnight at neutral conditions (dis. water, $\mathrm{pH}$ : 6.3) and room temperature. The successful $\mathrm{Fe}^{3+}$-complexation could firstly be confirmed by the dark coloration of the microgel solution (Figure 46a). In comparison, the experiment was performed for a microgel reference without tannic acid (Figure 46b). In this case, the microgel aqueous dispersion turned yellowish corresponding to the color of the added $\mathrm{FeCl}_{3}$-solution, but it did not show further color changes related to 
the formation of $\mathrm{Fe}^{3+}$-complexes. The difference between both samples can be visualized clearly.

As previously mentioned, the VCL-to-TA-ratio was varied systematically in order to determine its influence on the material properties (Chapter 3). In relation to metal complexation, it is important to point out that the increase of the amount of TA within the microgels leads to an increment of the surface negativity (Mob) at a pH of 6.3. This indicates the presence of the partial deprotonated TA in the polymeric network and the successful content variation. Thus, the $\mathrm{Fe}^{3+}$-loading was performed for 4 PVCL/TAmicrogels with varied TA-amounts. The loaded Fe-amount was determined indirectly via UV/Vis. Herein, the samples were centrifuged after the loading experiment, and the remaining iron quantity left in the supernatant was treated with salicylic acid for further complexation. A direct measurement of the $\mathrm{Fe}^{3+}$-content within the microgels was not possible because of signal overlapping of PVCL and the TA/ $\mathrm{Fe}^{3+}$-complex. Figure 46c displays the obtained results graphically. A correlation between the amount of tannic acid and the loaded mass of Fe [ $\mu \mathrm{g}]$ per microgel [mg] can be noticed directly. The higher the fraction of TA, the higher the $\mathrm{Fe}^{3+}$-amount loaded within the microgels. As expected, the TA-content shows a clear effect on the complexation degree. The amount of iron can be successfully varied, depending only on the VCL-to-TA-ratio. In addition, the measured data is compared to a negative reference (covalently crosslinked VCL/BIS-microgels, TA: 0.0 mol\%). The microgels without tannic acid cannot be used for iron complexation, which confirms the decisive role of the polyphenol in metal ion transport.

\section{EDX of Fe-loaded PVCL/TA-microgels:}

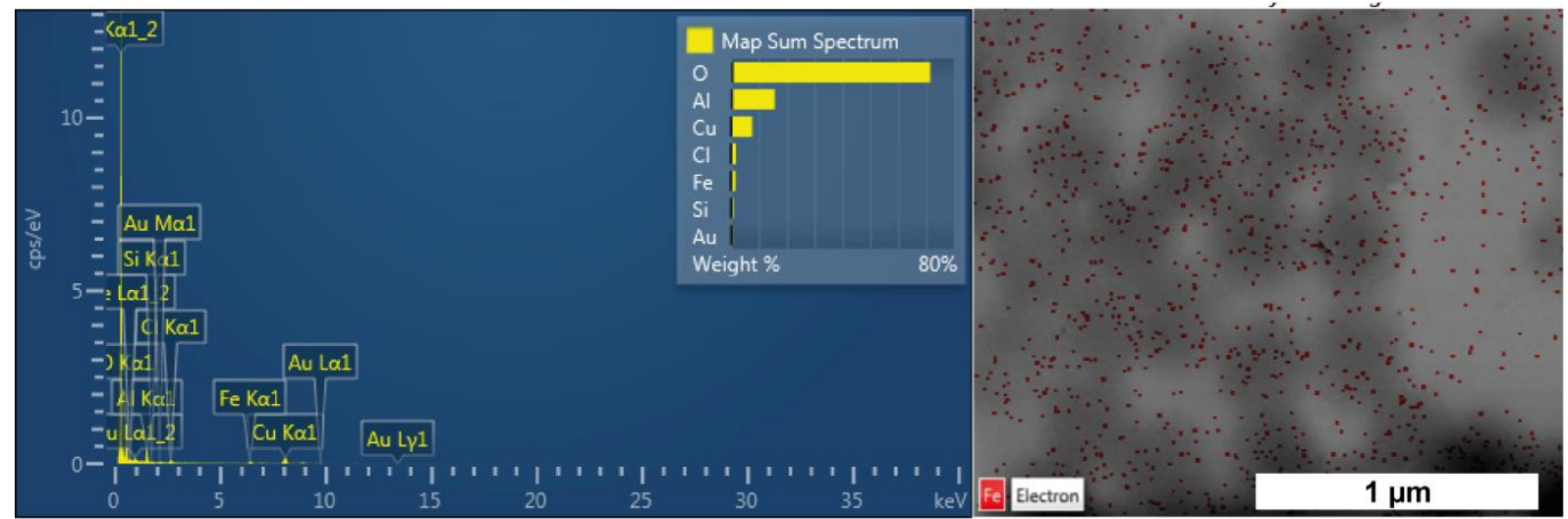

Figure 47. Elemental analysis of $\mathrm{Fe}^{3+-}$ loaded PVCL/TA-microgels (TA: $1.29 \mathrm{~mol} \%$ ). Left: STEM-image with elemental mapping. Right: EDX-spectrum. 
Moreover, one loaded sample (PVCL/TA-microgels, TAreal: 1.29 mol\%) was analyzed by elemental mapping and Energy-dispersive X-ray spectroscopy (EDX) (Figure 47), where the presence of the iron inside the microgels was indicated qualitatively. The elemental mapping visibly suggests the successful incorporation of Fe into the supramolecular polymeric network. Also, the EDX-spectrum sustains these results. Hereby, the corresponding signal for iron can be recognized $\left(\mathrm{K}_{\alpha}: 6.405 \mathrm{keV}\right)$. Besides, other elements such as oxygen, aluminum, copper, and chlorine were measured. Oxygen is not only present in the nanoparticles but is also a main component of the microgels. Copper and aluminum were analyzed due to the used TEM-grids, and chlorine due to the usage of $\mathrm{FeCl}_{3}$ as a precursor. Furthermore, these results are qualitative and do not define the Fedistribution inside the microgels. It is also important to note that the oxidation state of the iron within the microgels has to be confirmed by adequate methods, such as X-ray photoelectron spectroscopy (XPS) and high resolution TEM. The STEM-images presented here are even though a hint that there is no formation of $\mathrm{Fe}^{0}$-nanoparticles and that the incorporated $\mathrm{Fe}$ is in the $3+$ complexation-state. In addition, the mapping reveals that a small fraction of the Fe-signals are not detected within the polymeric particles. This can be a result of residuals or impurities. In conclusion, this qualitative study indicates visually that the iron complexation takes place in the microgels.

a.) Fe-release from PVCL/TA-microgels:

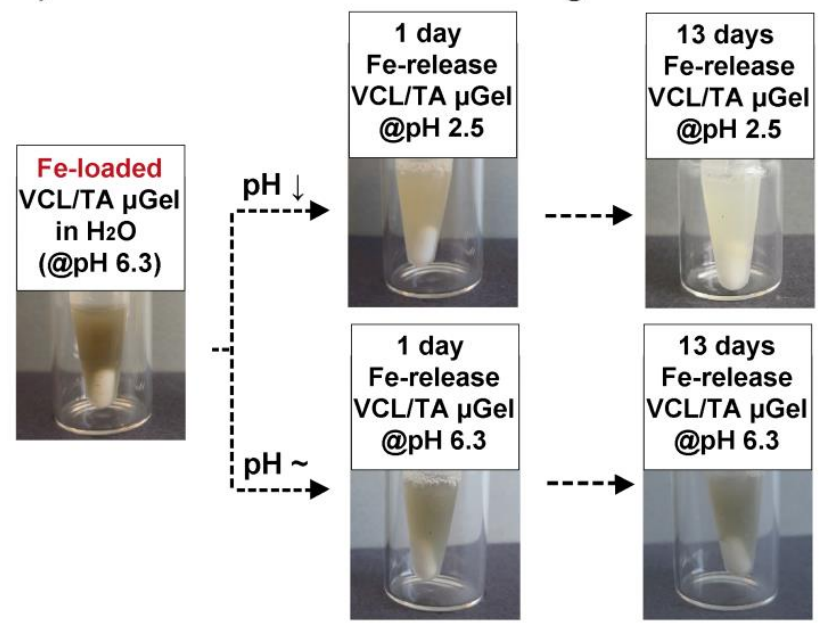

b.) Fe-release degree vs. time:

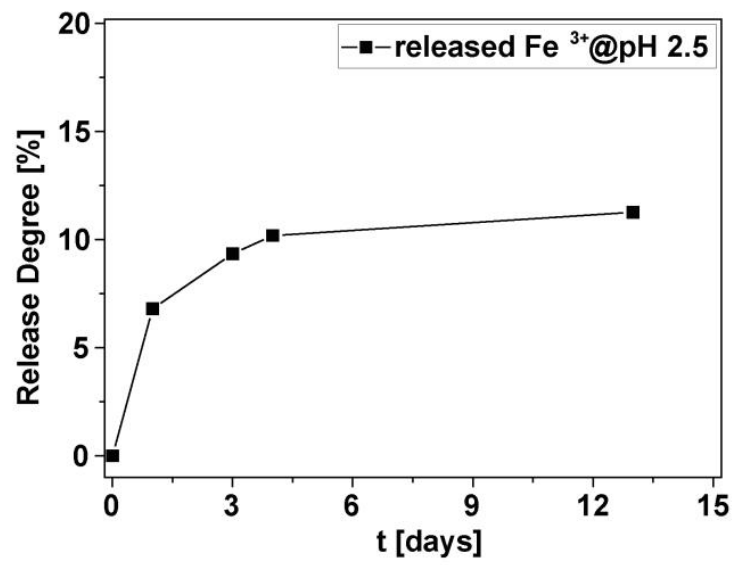

Figure 48. pH-controlled release of $\mathrm{Fe}^{3+}$ from PVCL/TA-microgels.

Finally, the pH-triggered release of $\mathrm{Fe}^{3+}$ was investigated (Figure 48). Herein, a sample of Fe-loaded PVCL/TA-microgels was treated with $\mathrm{HCl}(10 \mathrm{mM}, \mathrm{pH} 2.5)$ to induce the decomplexation and the simultaneous delivery of $\mathrm{Fe}^{3+}$ from the microgel network. As a 
control, a reference sample was treated with dis. $\mathrm{H}_{2} \mathrm{O}$ for the same period of time. Firstly, the acidic treatment leads to a visual color change of the sample (Figure 48a). After 13 days, the sample at a pH of 2.5 returns almost completely to the white/milky state of the microgel solution before loading. This suggests the release of the metal ions due to protonation of the tannic acid. Moreover, Figure 48b displays the cumulative calculation of the release degree in dependency of the time. Herein, the treated samples were centrifuged after various time periods and the supernatants were separated and analyzed by UV/Vis. The obtained values indicate the successful delivery of Fe-ions under acidic conditions. Still, the calculated results are lower than expected from the optical observations. A possible explanation is the loss of material after various centrifugation steps. This would lead to a systematic deviation during the study. By means of reproducibility and accuracy, this investigation should be optimized. Nevertheless, it can be concluded that the PVCL/TA-microgels can serve as carriers for the transport of Fe-ions. This report offers a promising candidate for biomedical applications, since as mentioned before, iron is an essential element in a large number of biological processes, and the design of responsive iron-vehicles as well as regulating systems is crucial in biomedicine.

\subsubsection{Incorporation of Other (Poly-) Phenolic Compounds}

In additional investigations, the proposed method for the synthesis of supramolecular microgels was utilized for the incorporation of further phenolic substances as active compounds and non-covalent crosslinkers. Dopamine, gallic acid and (+)-catechin were selected for these experiments due to their significance in biological activities, as well as their antibacterial, anticancer, and antioxidant properties. Figure 49 illustrates the chemical structures of the phenols.

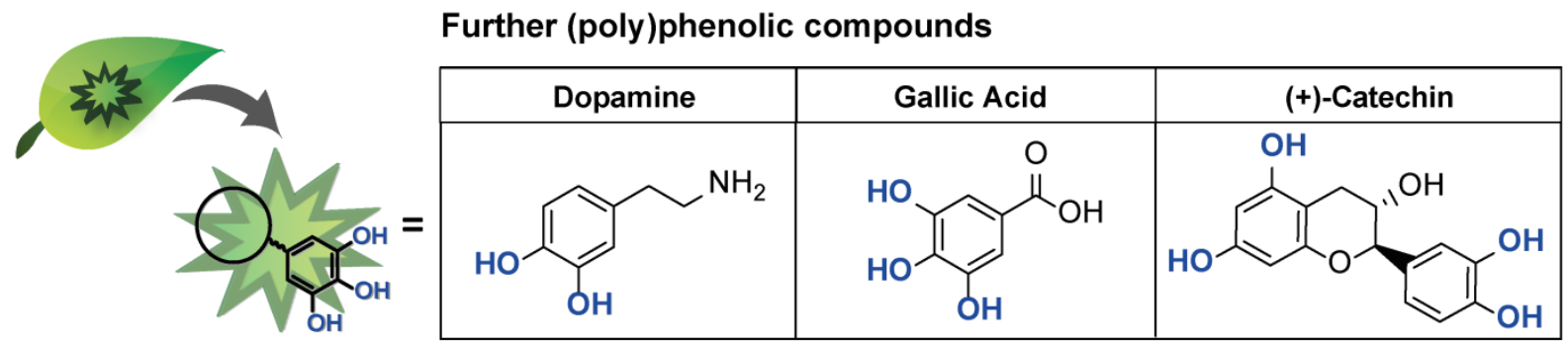

Figure 49. Phenolic derivatives as crosslinkers for the synthesis of supramolecular microgels. 
In contrast to tannic acid, the structures of these compounds possess only single or double phenolic groups. Their incorporation as non-covalent crosslinkers was applied in this thesis additionally as a model to determine the necessity of multiple phenolic rings for supramolecular crosslinking. The synthesis was carried out analogically to the optimized synthesis route described in chapter 3. According to the previously reported method, the different phenolic derivatives were added directly to the reaction after 15min of VCL-polymerization (Figure 50).

1.) Polymerization of $\mathrm{VCL}$

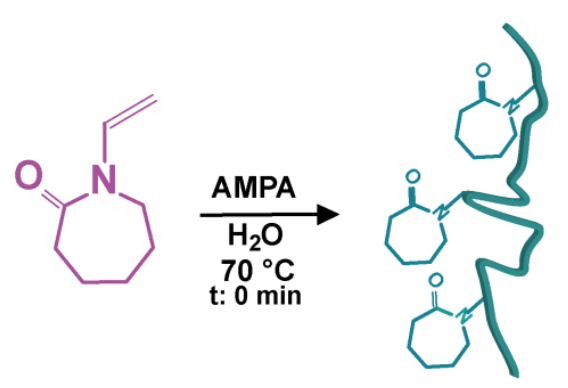

2.) Addition of phenol

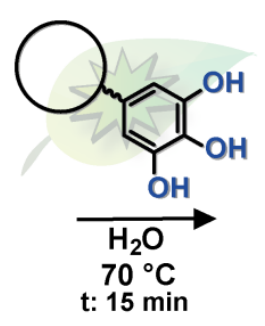

3.) Formation of supramolecular structures

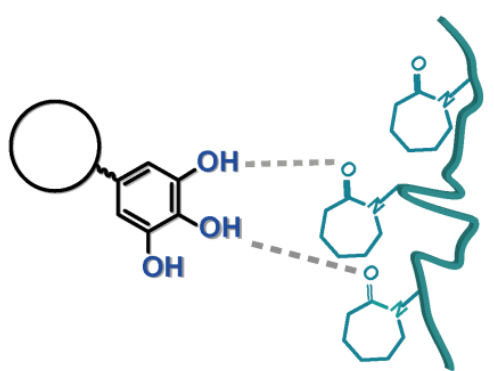

Figure 50. Synthesis route for the incorporation of various phenolic derivatives into microgels as supramolecular agents.

The VCL-to-phenol molar ratio was varied systematically for each component between 0.5 and $10 \mathrm{~mol} \%$. Herein, the primary step was the determination of the long term stability on the interactions between the phenolic compounds and PVCL. This analysis was performed visually depending on the stable turbidity of the PVCL/phenol-solutions after the cooling down. Table 10 summarizes the used molar fractions and the obtained observations related to the formation of non-covalent interactions. As described in chapter 2, the solubility of PVCL in $\mathrm{H}_{2} \mathrm{O}$ is temperature dependent (Figure 5). At room temperature, the polymer chains are in a relaxed state. However, an increase of the temperature above the LCST of PVCL leads to an increase of the polymer-to-polymer interactions that leads into chain collapse and the formation of polymer coils. This process is reversible, meaning that the polymer is able to return to the soluble state after cooling down. In this work, the stabilizing effect of tannic acid on the PVCL coil structures has been demonstrated. The principle of the formation of the supramolecular PVCL/TA-microgels relies on the strong and stable non-covalent interactions between PVCL and the polyphenolic macromolecule. In the presence of tannic acid, the PVCL 
chains maintain their collapsed state and therefore, the aqueous solution remains turbid after cooling down. The turbidity at room temperature after the microgel synthesis is the first indicator for the successful formation of the supramolecular structures.

Table 10. Formation of supramolecular structures by physical interactions between PVCL and phenolic compounds.

\begin{tabular}{|c|c|c|c|c|c|l|}
\hline $\begin{array}{c}\text { Phenolic } \\
\text { compound }\end{array}$ & 0.5 mol\% & 1 mol\% & 2 mol\% & 4 mol\% & 10 mol\% & description \\
\hline Dopamine & $x$ & $x$ & - & $x$ & $x$ & no structure \\
\hline Gallic Acid & $x$ & $x$ & $\checkmark$ & $\checkmark$ & $\checkmark$ & $\begin{array}{l}\text { stable } \\
\text { structures for } \\
\text { short time }\end{array}$ \\
\hline $\begin{array}{c}\text { (+)- } \\
\text { Catechin }\end{array}$ & - & $x$ & - & $\checkmark$ & $\checkmark$ & $\begin{array}{l}\text { stable } \\
\text { structures for } \\
\text { long time }\end{array}$ \\
\hline TA & $\checkmark$ & $\checkmark$ & $\checkmark$ & $\checkmark$ & - & $\begin{array}{l}\text { stable } \\
\text { interactions } \\
\text { for long time }\end{array}$ \\
\hline
\end{tabular}

The results of the incorporation of the various phenolic derivatives into microgels as supramolecular agents were firstly analyzed in view of the observed turbidity during and after the polymerization. Contrary to the obtained results for TA, the replacement of tannic acid with dopamine does not show a similar effect. All dopamine-based solutions became clear at room temperature after the microgel synthesis independent of the phenolic concentration. A further analysis by STEM confirmed the absence of microgels and thus, the deficiency of dopamine as crosslinking agent for this method. However, this observation was expected due to the lack of multi-phenolic units in the dopamine structure. Moreover, gallic acid showed similar results. Only the batches with a high GAfraction of 2, 4 and 10 mol\% remained turbid after cooling down, suggesting the formation of non-covalent structures between polymer and phenol. Nonetheless, these non-covalent interactions were not stable for a long term. After a short period of time ( 1 day) all the solutions became clear and the microscopic measurements confirmed the lack of microgels. 
PVCL-to-(+)-Catechin interactions:

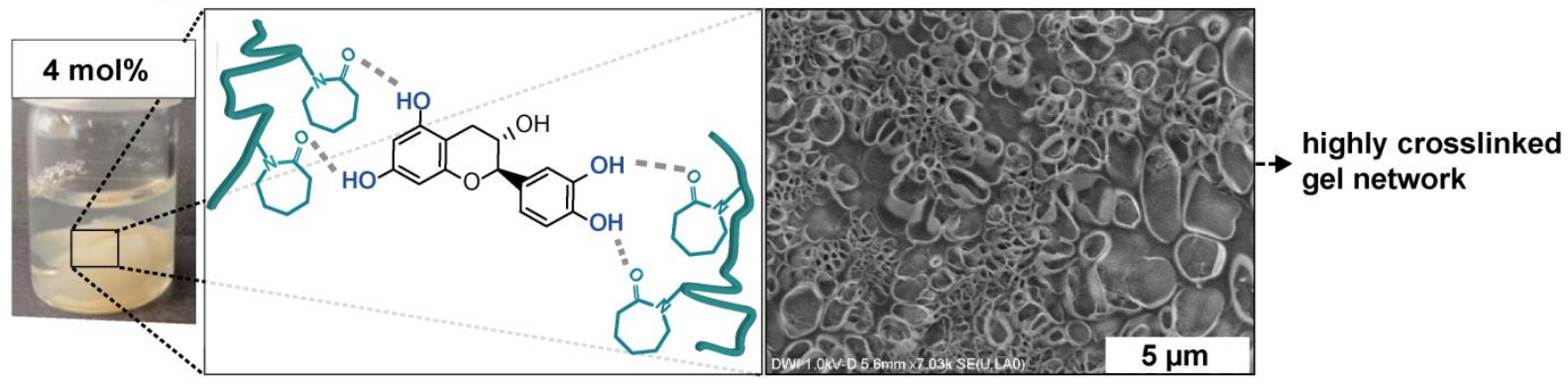

Figure 51. Non-covalent composite produced by interactions between (+)-catechin and PVCL.

$(+)$-Catechin was the only phenolic derivative that showed the formation of long term stable interactions with PVCL in this study. The turbidity of the samples containing 4 and $10 \mathrm{~mol} \%$ of this phenol was constant at room temperature independent of the time. Moreover, precipitation of the non-covalent structures was observed, resulting in the formation of jelly-like composites. By cryoSEM, the product was characterized and the production of a highly crosslinked gel network was confirmed (Figure 51). The results obtained in this section confirm the necessity of multi-phenols for the formation of crosslinked structures. Based on this positive outcome, (+)-catechin was considered as a suitable crosslinking agent for the synthesis of supramolecular microgels.

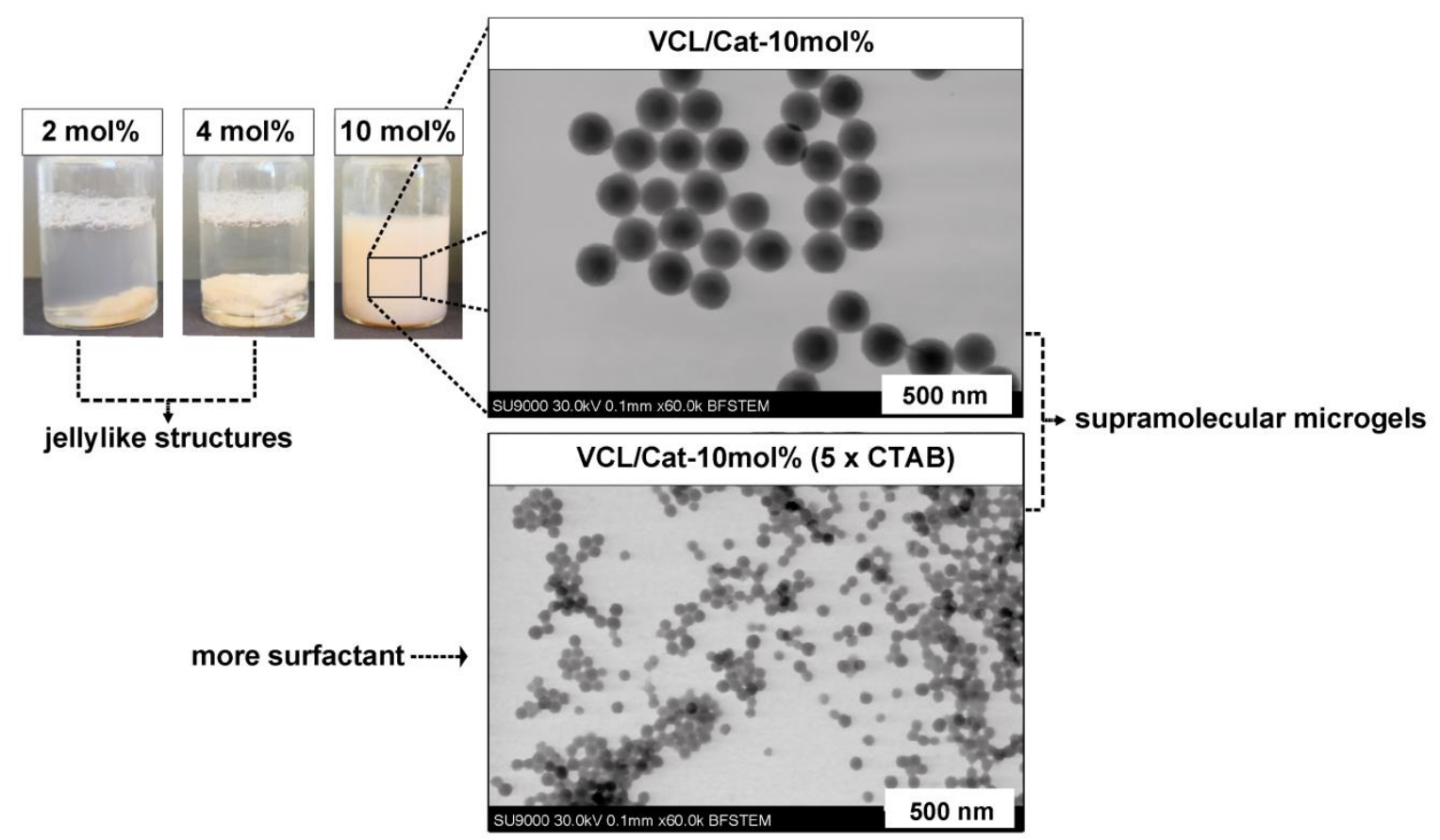

Figure 52. Production of supramolecular microgels based on (+)-catechin as physical crosslinker. 
The approach was optimized and therefore, the addition of (+)-catechin was performed dropwise to improve the colloidal stability and hinder strong precipitation. After the optimization, the successful synthesis of stable microgels was achieved for a $(+)$-catechin content of $10 \mathrm{~mol} \%$ (Figure 52). In this case, a stable colloidal system was obtained and the presence of highly monodisperse and well-defined polymeric particles was confirmed via STEM. In the optimized synthesis approach, CTAB is added to the monomer solution as an additional stabilizing agent (Chapters 2 and 3). A diminution of the particle size could be additionally observed by increasing the surfactant amount used during the microgel synthesis.

a.) Molecular structures:

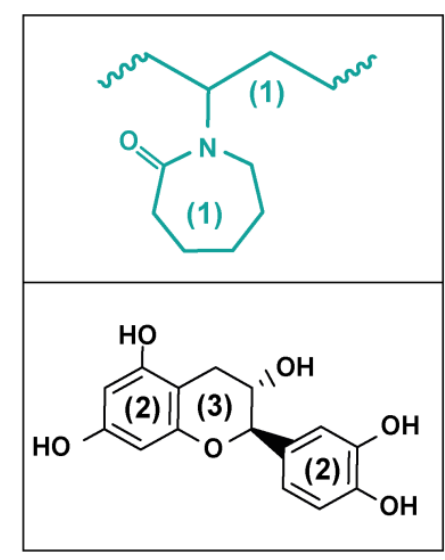

b.) Raman spectra:

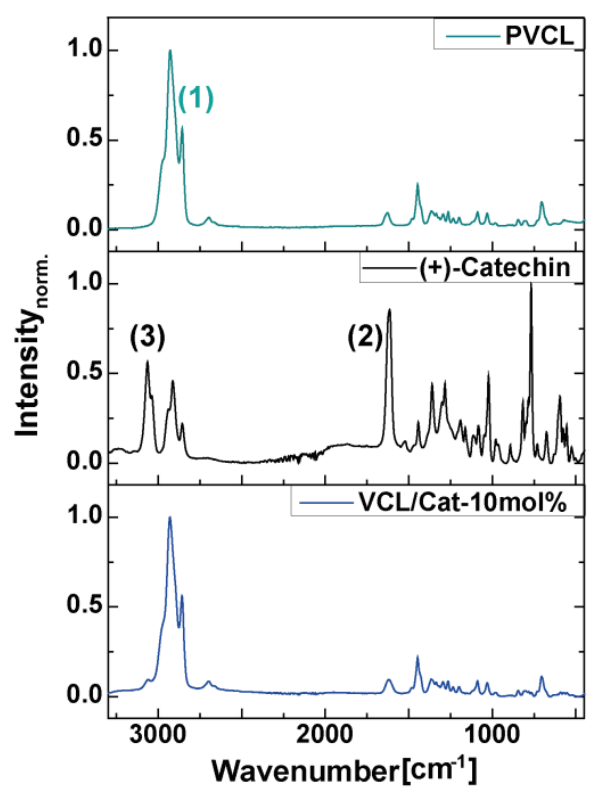

Figure 53. Determination by Raman spectroscopy of the incorporation of (+)-catechin as physical crosslinker into PVCL-microgels.

The incorporation of (+)-catechin as supramolecular crosslinking agent into the PVCLmicrogels was characterized by Raman spectroscopy. In Figure 53, the molecular structures of the references: PVCL and TA, as well as their Raman spectra in comparison to the data corresponding to the microgels are depicted. Unfortunately, the representative signals of both components are present at similar wavenumbers, hindering the differentiation of both building blocks within the microgels. Nonetheless, one can confirm qualitatively the successful incorporation of the phenolic molecule within the polymeric network. The microgel curve (VCL/TA-10mol\%) shows a signal at a wavenumber of $3065 \mathrm{~cm}^{-1}$, which correlates with the aliphatic C-H vibrations of 
$(+)$-catechin (3). This result indicates that the microgels are composed of polymer chains and the phenolic compound. The chemical composition has to be studied in detail in further experiments to quantify the real fraction of $(+)$-catechin within the polymeric materials.

a.) Size and polydispersity:

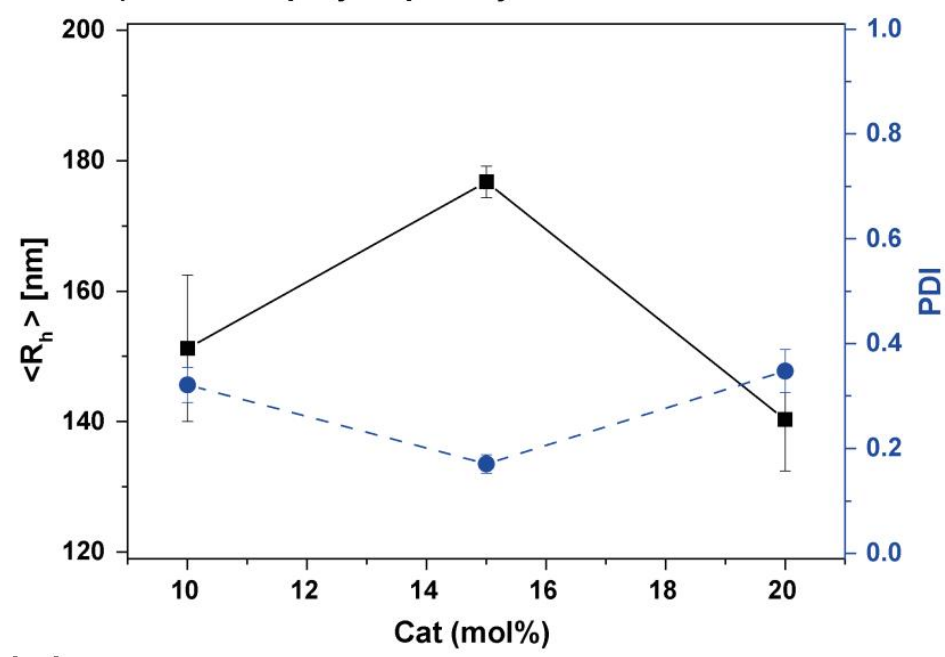

b.) Microgel morphology:

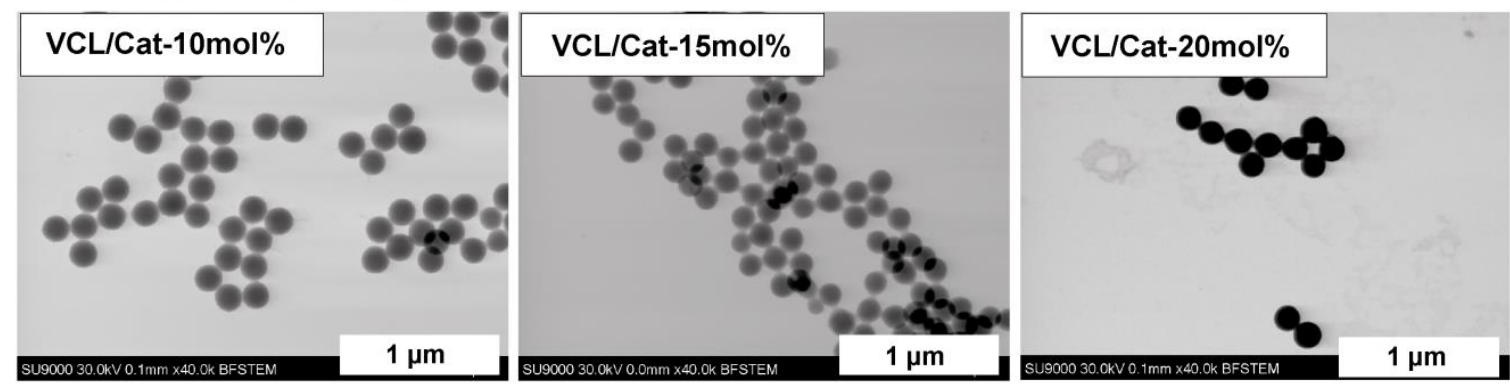

Figure 54. Systematic variation of the (+)-catechin-content during the microgel synthesis.

The theoretical (+)-catechin fraction was varied in further experiments to study its effect on the particle size and crosslinking density. The phenol content was thus increased up to $20 \mathrm{~mol} \%$. Figure 54 presents the characterization results for the microgel size and polydispersity and morphology in dependence of the molar fraction of $(+)$-catechin. The microscopic images reveal in all cases the production of monodisperse spherical microgels. However, there is no evident effect of the (+)-catechin-content on the microgel size either in aqueous solution (Figure 54a) nor in the dried state (Figure $54 \mathrm{~b})$. The DLS results show hydrodynamic radii between 140 and $180 \mathrm{~nm}$ for the 3 PVCL/Cat-systems, which are higher than expected. Additionally, the DLS-measured PDI values between 0.2 and 0.4 suggest high polydispersity, which is contradictory to the microscopic observations. The unforeseen DLS results are a hint for the formation of 
microgel clusters due to the well-known adhesive properties of catechol derivative materials. ${ }^{172}$ The aggregation effect between the microgel particles leads to imprecise $\mathrm{R}_{\mathrm{h}}$ and PDI results.

Lastly, the supramolecular conformation of the PVCL/Cat-microgels (Cat theo: 10 mol\%) was confirmed by their pH-sensibility (Figure 55). Aqueous microgel solutions $\left(10 \mathrm{mg} \cdot \mathrm{mL}^{-1}\right)$ were therefore prepared either with $\mathrm{HCl}(10 \mathrm{mM})$ or $\mathrm{NaOH}(10 \mathrm{mM})$ to adjust the $\mathrm{pH}$ value to 2.5 or 11.7. At a pH below $6.3\left(\mathrm{H}_{2} \mathrm{O}\right)$, the microgel solutions do not show any changes and remain stable. The increase of the $\mathrm{pH}$ to basic conditions however leads to the disintegration of the polymer network. This can be visualized clearly by the color alternation.

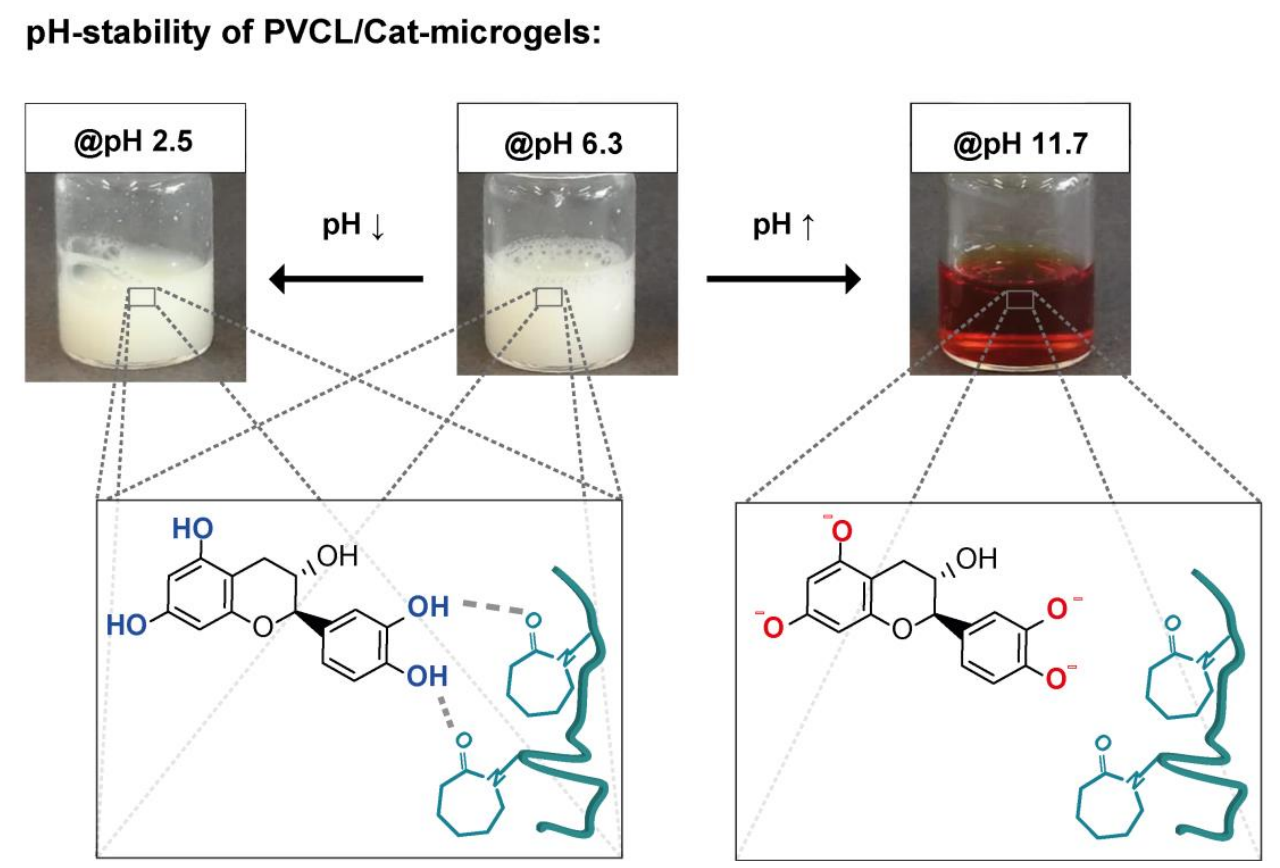

Figure 55. . pH-Stability of the supramolecular PVCL/Cat-microgels.

The microgel solution becomes clear/reddish indicating the break-down of the physical crosslinks and the simultaneous delivery of the $(+)$-catechin molecules from the polymer network. This outcome fulfills the expectation and correlates with the tannic acid studies presented in the previous chapters. In conclusion, the facile method proposed in this thesis allows not only the incorporation of TA as a physical crosslinker, but also the utilization of further multi-phenolic derivatives like $(+)$-catechin for the production of pH-sensitive supramolecular microgels. 


\subsubsection{Encapsulation of Antibacterial Alkyl Gallates}

Due to the previously mentioned problematic in Brazil related to the citrus production, an international research project called ProPlanta was proposed and accepted in 2017 by the São Paulo Research Foundation (FAPESP) and the German Federal Ministry of Education and Research (BMBF). Herein, the project goal is to develop a valid long-term approach to prevent the disease citrus canker caused by the bacterium X. Citri. This plant infection leads to defoliation, dieback and early fruit drop, affecting strongly the citrus agriculture in Brazil, which is the largest orange producer worldwide. The investigations are therefore aimed at plant protection, utilization of biocompatible materials, and triggered release of antibacterial compounds. If successful, the biocompatible multifunctional system should replace the usage of the current applied pesticides and toxic substances.

The research platform consists of 3 main parts (Figure 56). The first step is the synthesis of alkyl gallates. These gallic acid derivatives with an aliphatic rest are antibacterial compounds that act potently against the X. Citri bacteria. Due to the alkyl function, the gallates are highly hydrophobic, so that a suitable transport system is necessary. This requirement is the reason for the second part of the project, which is the encapsulation of the gallates into biocompatible and responsive compartments. In this case, responsive and degradable microgels should serve as suitable carriers. Finally, the last task of the project is the coating of the citrus plants by anchor peptide technologies reported already by Schwaneberg and coworkers. ${ }^{159,173}$

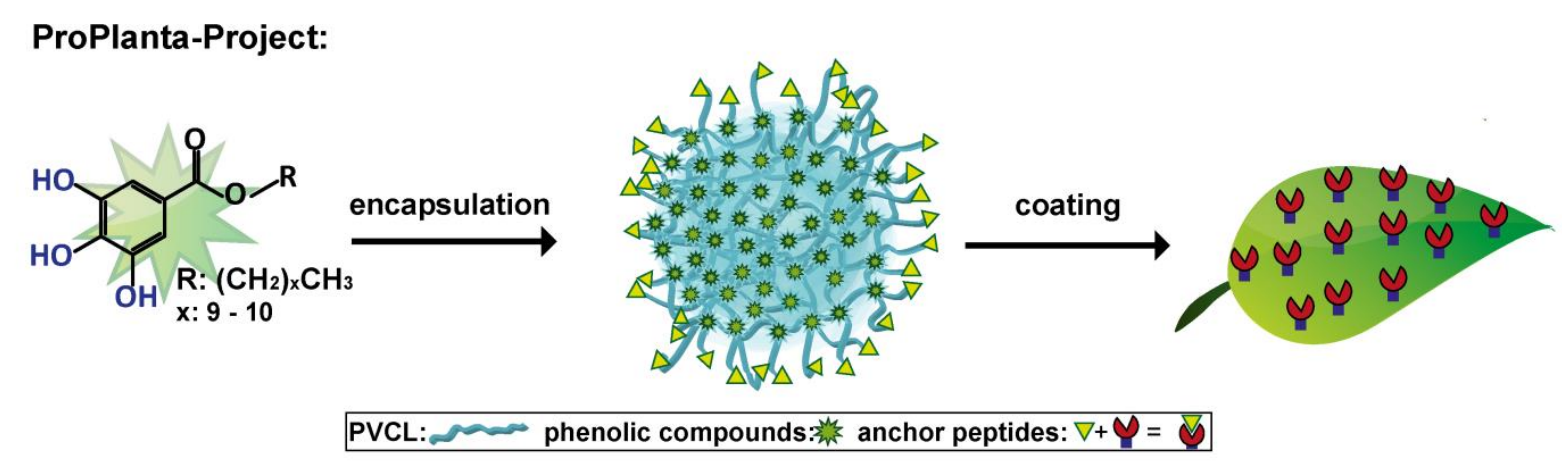

Figure 56. Graphic illustration of the ProPlanta project.

It is important to remark that this is an interdisciplinary project in which 4 different research groups are associated. From Brazil, the research groups of Prof. L. Regasini and 
Prof. H. Ferreira are responsible for the synthesis of the alkyl gallates and the investigations of their antibacterial action respectively. ${ }^{167,169}$ The studies related to the encapsulation and microgel design are assigned to our research group (Prof. A. Pich), and the scientific group of Prof. U. Schwaneberg is responsible for the peptide functionalization and plant coating. In this thesis, the results about the microgel synthesis and gallate encapsulation are discussed, as well as preliminary antibacterial studies are presented.

First of all, proof-of-principle experiments were performed in view of gallate encapsulation into supramolecular microgels. Herein, the well-established approach for the synthesis of supramolecular microgels was utilized as reported previously in chapter 3. The non-covalent responsive microgels composed of PVCL chains and the bioactive polyphenolic compound TA are excellent candidates in view of the production of biodegradable and biocompatible transport systems. Due to the fact that tannic acid as well as the gallates are gallic acid derivatives, their chemical structures are based on the same phenolic unit. This enables similar interactions with the PVCL chains and allows the incorporation of the antibacterial components as additional non-covalent crosslinkers. The gallate (G10) used for these experiments was synthesized, purified and characterized by the research group of Prof. Luis Regasini at the State University of São Paulo in São José do Rio Preto, Brazil.

As mentioned previously, alkyl gallates show a high hydrophobicity due to their aliphatic chain. Therefore, different solvents were used for their addition during the microgel synthesis. The first investigations were performed with ethanol. Herein, the antibacterial compound G10 (aliphatic chain: $\left.\left(\mathrm{CH}_{2}\right)_{9} \mathrm{CH}_{3}\right)$ was dissolved in the alcohol and added to the aqueous synthesis mixture after $15 \mathrm{~min}$ of VCL polymerization, accordantly to the already presented method. The antibacterial component was added during the microgel synthesis instead of the crosslinker TA in first place, and its molar ratio was varied. The obtained microgels are exhibited in Figure 57. 
Incorporation of $\mathrm{G10}$ as (co-)crosslinker:

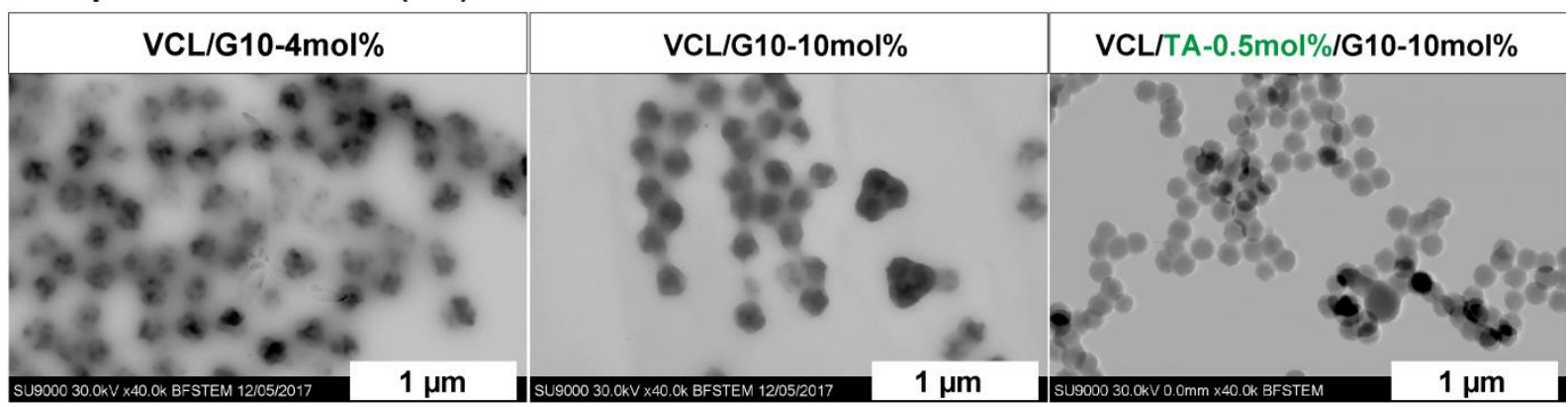

Figure 57. Incorporation of G10 as non-covalent (co-) crosslinker. Left and middle: microgels containing G10 as single crosslinker. Right: microgels containing a combination of TA and G10 as multiple crosslinkers.

The microgel samples based only on G10 as crosslinking agent (VCL/G10-4mol\% and $10 \mathrm{~mol} \%$ ) revealed small particle sizes, and a strong tendency to agglomeration. Nevertheless, the formation of polymeric particles demonstrates that it is possible to encapsulate the hydrophobic and antibacterial gallates within a biocompatible polymer (PVCL) in aqueous solutions as single crosslinkers. Moreover, the strong contrast of the STEM images suggests the formation of core-shell structures. Due to the high hydrophobicity of G10, it can be assumed that the amphiphilic polymer PVCL act as stabilizing agent in aqueous media. This would lead to the concentration of the hydrophobic compound in the microgel core and thus higher crosslinking density. This correlates with the observed decreased microgel size and increased trend to form clusters. A comparison of this observation to the previous results for the incorporation of pure gallic acid as crosslinker is also very interesting. Contrary to the PVCL-GAinteractions, the non-covalent forces between PVCL and G10 remain stable after a long period of time. This suggests the significant effect of the aliphatic rest for the stability of the system. One can therefore propose that the hydrophobicity of the gallate increases the strength of the physical interactions between polymer and phenolic derivative due to stabilizing effects.

Furthermore, additional supramolecular microgels were produced using a combination of TA (0.5 mol\%) and G10 (10 mol\%) as multiple crosslinker system. The size, size distribution and the morphology of the synthesized microgels were determined by STEM and DLS. Herein, the additional incorporation of TA to the gallate-based system results in the synthesis of monodisperse and well-defined microgels (Figure 57-right). This result can be compared to the system containing only $0.5 \mathrm{~mol} \%$ of TA (Chapter 3 ). 
The combination of both gallic acid derivatives as crosslinkers leads to a decrease of the particle size and indicates an improvement of the monodispersity. Still, the DLS measurements do not corroborate this statement. The calculated PDI value for the sample based on both crosslinkers suggests polydisperse materials (Table 11. VCL/TA$0.5 \mathrm{~mol} \% / G 10-10 \mathrm{~mol} \%)$. The hydrophobicity and strong tendency of the G10-based microgels to agglomerate can explain the unexpected result, since DLS measurements are carried out in aqueous solution. Lastly, it can be concluded that the incorporation the utilization of TA leads to higher yields.

Table 11. Hydrodynamic radii and PDI values calculated by DLS and gravimetric yield of the first gallatebased microgels.

\begin{tabular}{|c|c|c|c|}
\hline Sample & VCL/G10-10mol\% & VCL/TA-0.5mol\%/G10-10mol\% & VCL/TA-0.5mol\% \\
\hline $\mathrm{R}_{\mathrm{h}}[\mathrm{nm}]$ & 240 & 185 & 215 \\
\hline PDI & 0.23 & 0.27 & 0.1 \\
\hline Yield [\%] & 80.2 & 99.0 & 96.9 \\
\hline
\end{tabular}

The chemical composition of the presented G10-containing microgels was characterized via Raman spectroscopy (Figure 58). In principle, the successful incorporation of the gallic acid derivatives can be confirmed qualitatively. The representative signal of PVCL $\left(2928 \mathrm{~cm}^{-1}\right)$ and the corresponding signals for the gallic acid derivatives (1600 and $1750 \mathrm{~cm}^{-1}$ ) are present in the synthesized microgels. However, due to the fact that tannic acid and G10 are both based on gallic acid units, they have very similar chemical structures. A differential selective chemical analysis to distinguish both components in microgel structure using conventional analytical methods is therefore rather challenging.

a.) Molecular structures:

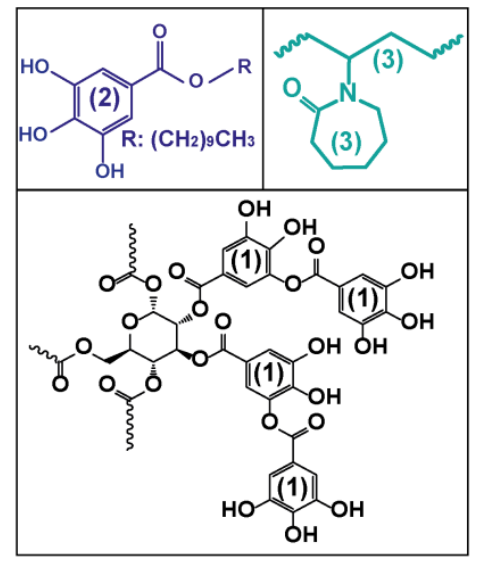

b.) Raman-spectra of the references and microgels:

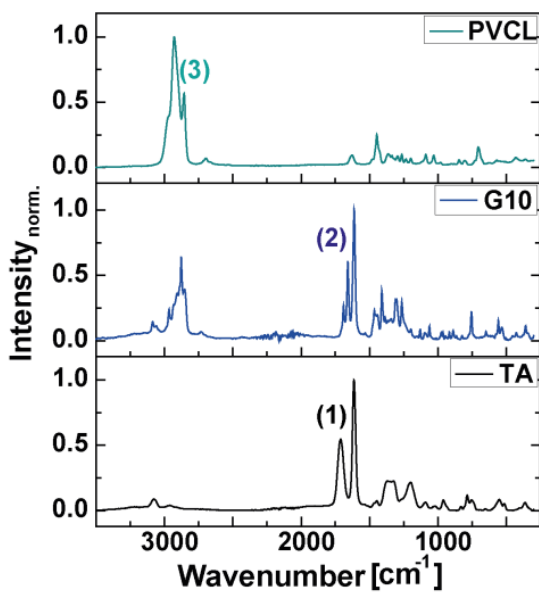

Figure 58. Study of the chemical composition of G10-based microgels by Raman spectroscopy. 
In addition, further experiments were carried out using dimethyl sulfoxide (DMSO) as co-solvent for G10 to improve its solubility and thus its incorporation. Herein, the method for the gallate addition was modified. The incorporation of G10 was performed drop by drop using a syringe pump for the flow rate control, and the addition time was varied from 5 to 60 min. Figure 59 displays the characterization results. The utilization of DMSO as gallate solvent leads to similar results as in the case of ethanol. High crosslinking density within the microgel core can be observed in all the cases. However, the time variation seems to influence the microgel formation positively (Figure 59a).

a.) Time variation for G10-addition (without TA):

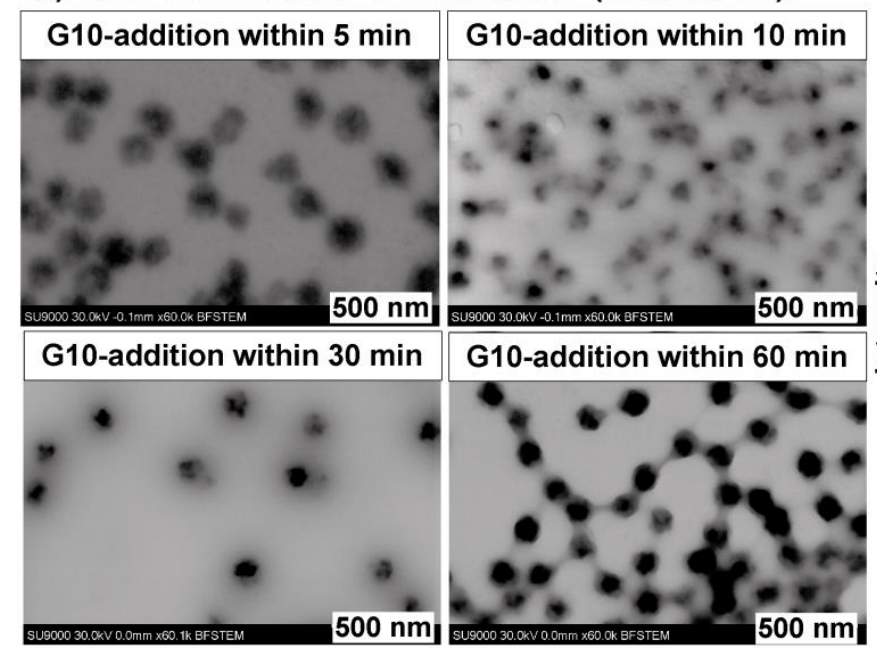

b.) Size distribution (without TA):

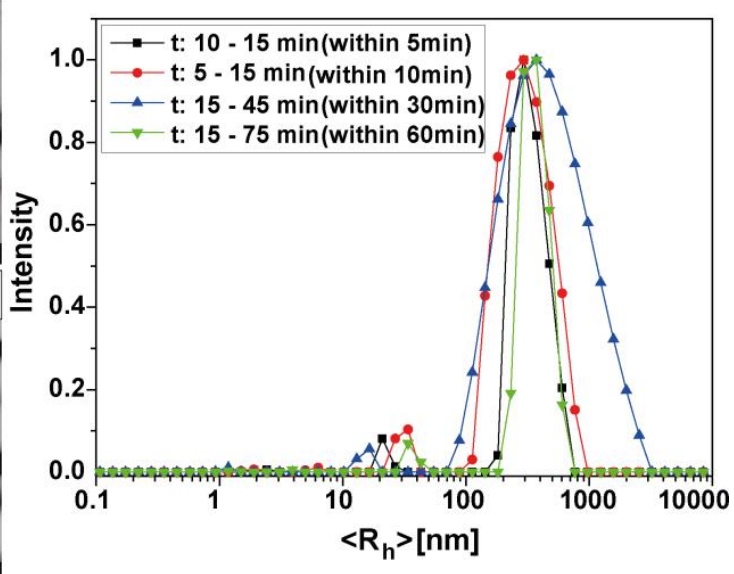

Figure 59. Optimization of the gallate G10 (10 mol\%) encapsulation into supramolecular microgels.

Furthermore, the form of addition (dropwise or direct) of the gallate and the time showed also an influence on the microgel structure. If the dropwise addition of G10 (10 mol\%) is carried out within a time period of 5 or $10 \mathrm{~min}$, the formation of precipitates was also observed after the microgel synthesis. However, these experiments do not reveal the formation of well-defined microgels. Instead, they show hydrophobic clusters within a polymer film (Figure 59a). Contrary to this effect, the G10 addition within a longer period of time (30 to $60 \mathrm{~min}$ ) leads to a decrease of the precipitation tendency of the product, increasing its colloidal stability. Herein, the microscopic characterization indicates the presence of separated microgel particles, even though these still tend to form agglomerates. The analysis of the particle size distribution calculated by DLS does not distinctly expose a definitive conclusion. One observes that a narrow size distribution was achieved by a longer time period for the G10 addition (60 min). However, similar results were obtained after the addition within a short time 
frame (5 min). As mentioned above the high hydrophobicity and strong tendency of the G10-based microgels to agglomerate in aqueous solution may affect strongly the DLS measurements. Nevertheless, the microscopic images indicate clearly that a better encapsulation and microgel formation can be achieved through a slower addition of the gallate. Time-variation experiments were therefore carried out also for the synthesis of microgels containing TA $(0.5 \mathrm{~mol} \%)$ in addition to G10 (2 mol\%). The corresponding STEM images are depicted in Figure 60.

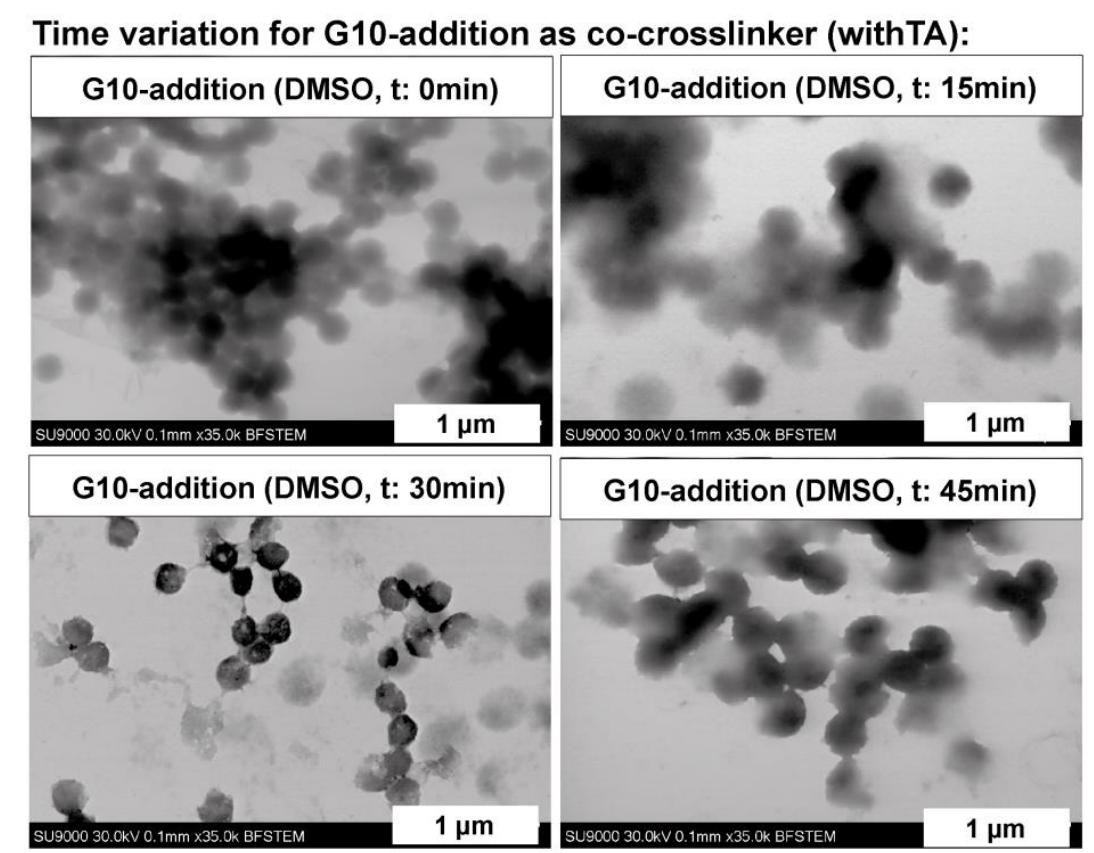

Figure 60. Time variation for G10-addition during the microgel synthesis using G10 (2 mol\%) as a cocrosslinker in combination with TA (0.5 mol\%).

Corresponding to the preliminary studies, the combination of TA and G10 as multiple crosslinkers improves the colloidal stability and the microgel formation. Moreover, similar to the previous time-variation experiments, longer periods of time affect positively the microgel synthesis in terms of agglomeration and formation of welldefined polymeric particles. The best results were obtained by the G10-addition within $30 \mathrm{~min}$ and thus, this parameter was chosen to produce supramolecular PVCL/TAmicrogels with varying G10 amount.

The variation of the gallate content within the supramolecular TA-based microgels strongly influences the morphology and the particle size (Figure 61). The higher the G10-amount incorporated, the smaller and denser the microgels become. This result correlates with the expectations, since the increase of the hydrophobic fraction of the 
system increases with the G10-content. In conclusion, the synthesis approach presented in this thesis is a suitable method that allows not only a supramolecular assembly, but also the encapsulation of hydrophobic components in a water-based system.

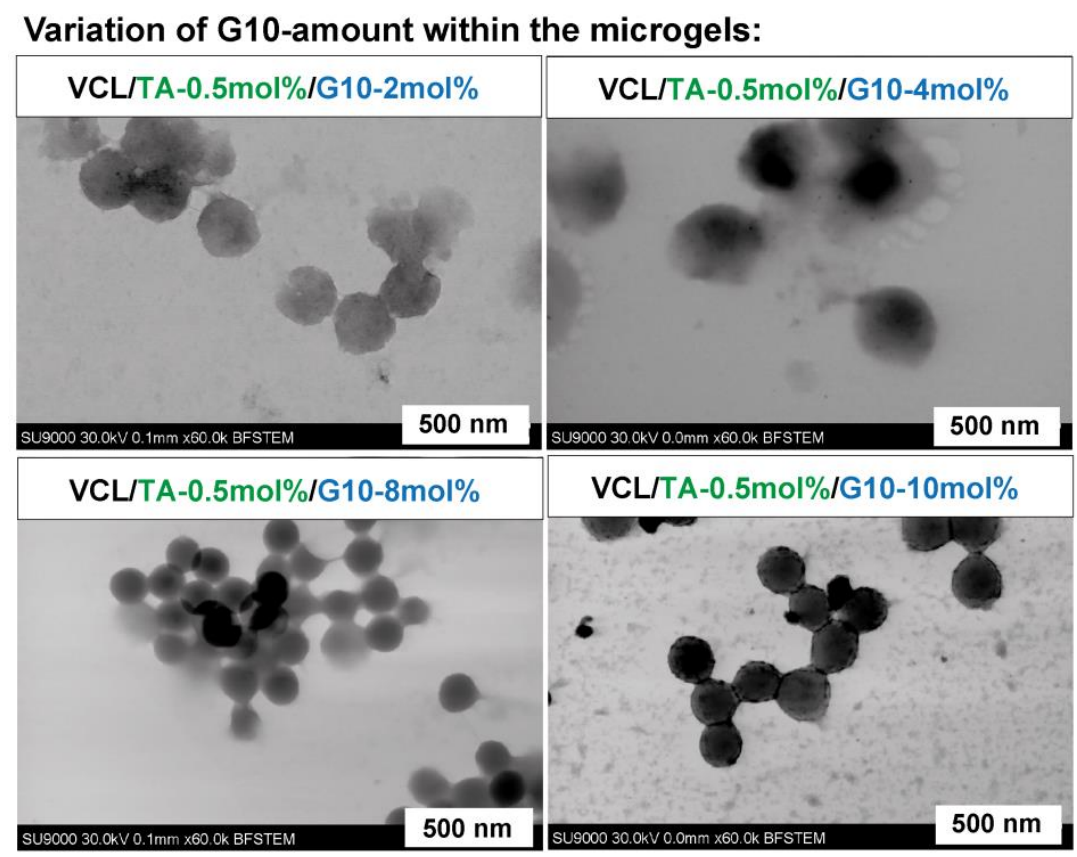

Figure 61. Variation of G10-amount within PVCL/TA-microgels.

In the end, the stability of the G10-based microgels was determined in DMSO. Therefore, various PVCL/TA/G10-microgel systems were dissolved in the organic solvent (10 $\mathrm{mg} \cdot \mathrm{mL}^{-1}$ ) and the solutions were characterized by STEM measurements (Figure 62). The complete disintegration of the microgel networks was corroborated in all cases. Due to the chemical structure of DMSO, the crosslinking agents TA and G10 interact strongly with it, promoting the break-down of the hydrogen bonds between both gallic acid derivatives and PVCL. Interestingly, the presence of DMSO during the microgel synthesis does not affect the microgel formation since the utilized volume is very small in comparison to the aqueous medium $(<1: 99$ Vol.-\%). This preliminary observation is an interesting hint that confirms the supramolecular conformation of the G10-loaded microgels. 
Stability of the microgels in DMSO:

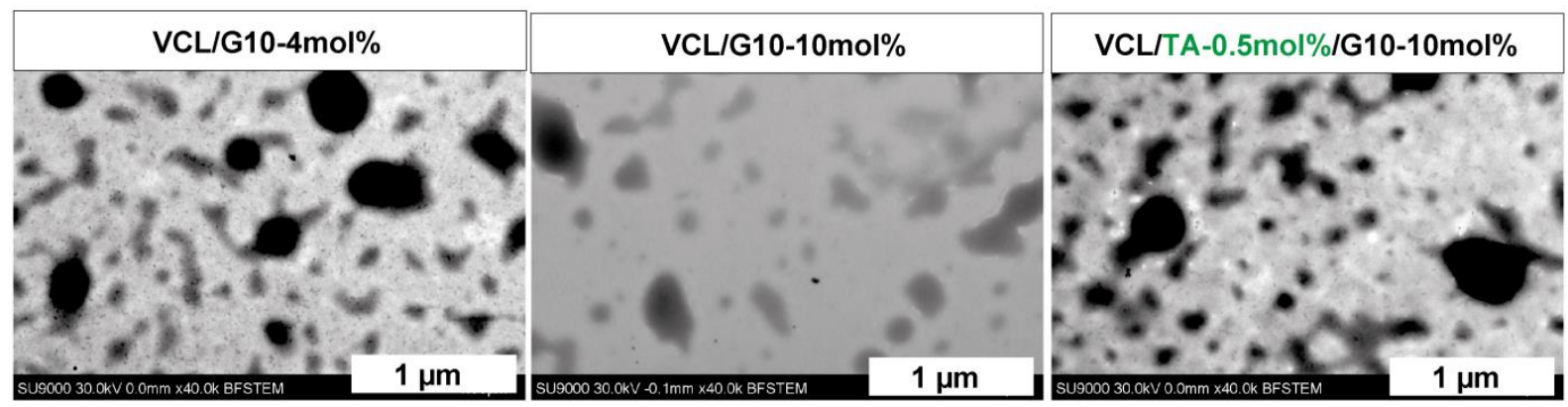

Figure 62. Stability of G10-based microgels in organic solvents.

In the last section of this doctoral research study, various supramolecular gallate-based microgels were selected for a preliminary antibacterial analysis. The antibacterial studies were performed at the State University of São Paulo at Rio Claro by Guilherme Dilarri from the research group of Prof. Henrique Ferreira. These results are reproduced with permission and after discussion with the collaboration partners.

In the first place, the antibacterial activity of pure tannic acid in comparison to the supramolecular microgels was characterized by resazurin microtiter assay (REMA). ${ }^{169}$ The utilization of this approach allows the monitoring of the bacterial cell respiratory activity in the presence of bioactive substances at different concentrations. Herein, 3fold analysis was performed for the determination of the minimum inhibitory concentrations (MIC): $\mathrm{MIC}_{50}$ (inhibition of $50 \%$ of X. Citri cells) and MIC 90 (inhibition of $90 \%$ of X. Citri cells). The cell inhibition was plotted against the concentration of the different compounds and the results are depicted in Figure 63a. The calculated values for the different MICs are summarized in Table 12. Its determination was conducted by polynomial regression and extrapolation of the obtained data. As a reference, the gallate G10 used for the microgel synthesis exhibits a MIC90 of $\sim 30 \mu \mathrm{g} \cdot \mathrm{mL}^{-1}\left(\mathrm{MIC}_{50}\right.$ : $\left.15.6 \mu \mathrm{g} \cdot \mathrm{mL}^{-1}\right) .169$

Table 12. MIC of pure TA and microgels calculated by REMA.

\begin{tabular}{|c|c|c|}
\hline Compound & MIC $_{\mathbf{5 0}}$ & MIC $_{\mathbf{9 0}}$ \\
\hline $\boldsymbol{T A}$ & $24.551 \mu \mathrm{g} \cdot \mathrm{mL}^{-1}$ & $99.925 \mu \mathrm{g} \cdot \mathrm{mL}^{-1}$ \\
\hline $\boldsymbol{V C L} / \boldsymbol{T A} \cdot \mathbf{0 . 5 m o l \%}$ & - & - \\
\hline $\boldsymbol{V C L} / \mathbf{G 1 0 - 1 0 m o l \%}$ & $4.635 \mathrm{mg} \cdot \mathrm{mL}^{-1}$ & $9.760 \mathrm{mg} \cdot \mathrm{mL}^{-1}$ \\
\hline $\begin{array}{c}\boldsymbol{V C L} / \boldsymbol{T A}-\mathbf{0 . 5 m o l \%} / \mathbf{G 1 0}- \\
\mathbf{1 0 m o l \%}\end{array}$ & $3.459 \mathrm{mg} \cdot \mathrm{mL}^{-1}$ & $13.111 \mathrm{mg} \cdot \mathrm{mL}^{-1}$ \\
\hline
\end{tabular}


Firstly, the REMA data of pure TA indicates that the polyphenol may improve the cell growth inhibition within the novel bioactive microgel system, and its utilization in higher amounts could lead to a reinforcement of the antibacterial properties of the materials. Still, it can be observed that the PVCL/TA-microgels do not show significant inhibition characteristics and no antibacterial activity could be detected. These results correlate with the expectations due to the fact that the TA-molar fraction of the system is very low (0.5 mol\%) and the polymer PVCL is biocompatible (main component of the system). On the other hand, the microgels containing G10 exhibit positive inhibition properties, even though the MICs are extremely high in comparison with pure G10. In ideal case, an antibacterial compound should inhibit the cell growth in a concentration below $100 \mu \mathrm{g} \cdot \mathrm{mL}^{-1}$ to be valuable for further applications.

a.) Cell growth inhibition of $X$. Citri in the presence of TA or supraolecular microgels:
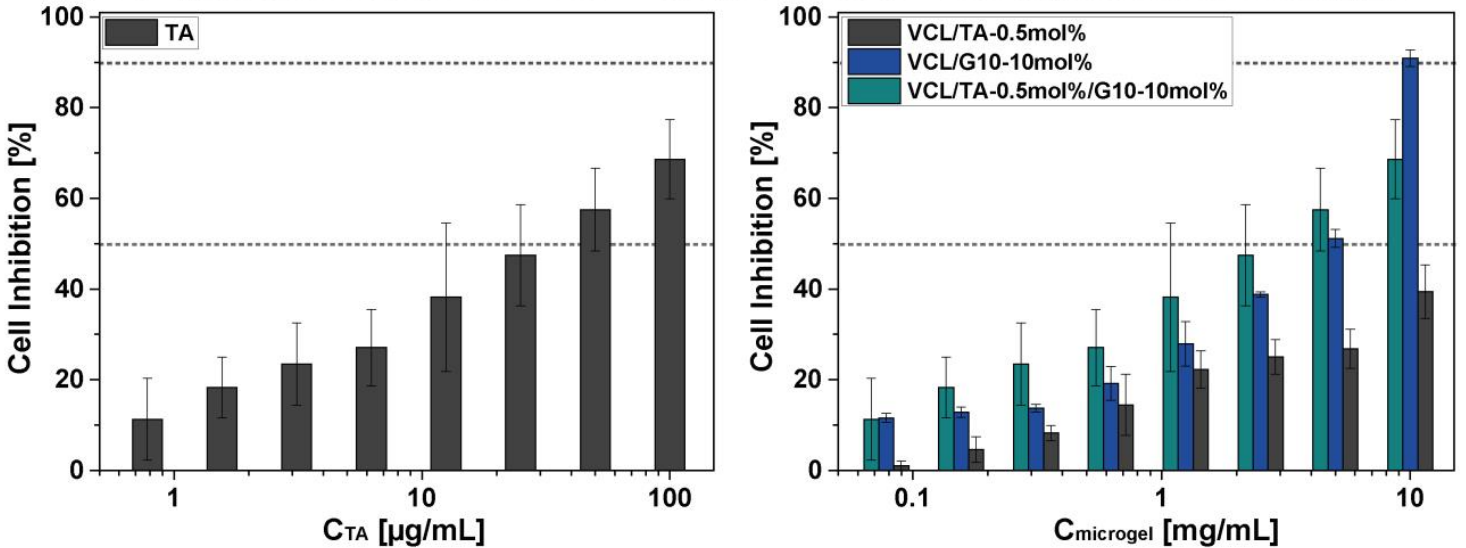

b.) Cell viability of $X$. Citri after treatment with TA or supraolecular microgels:
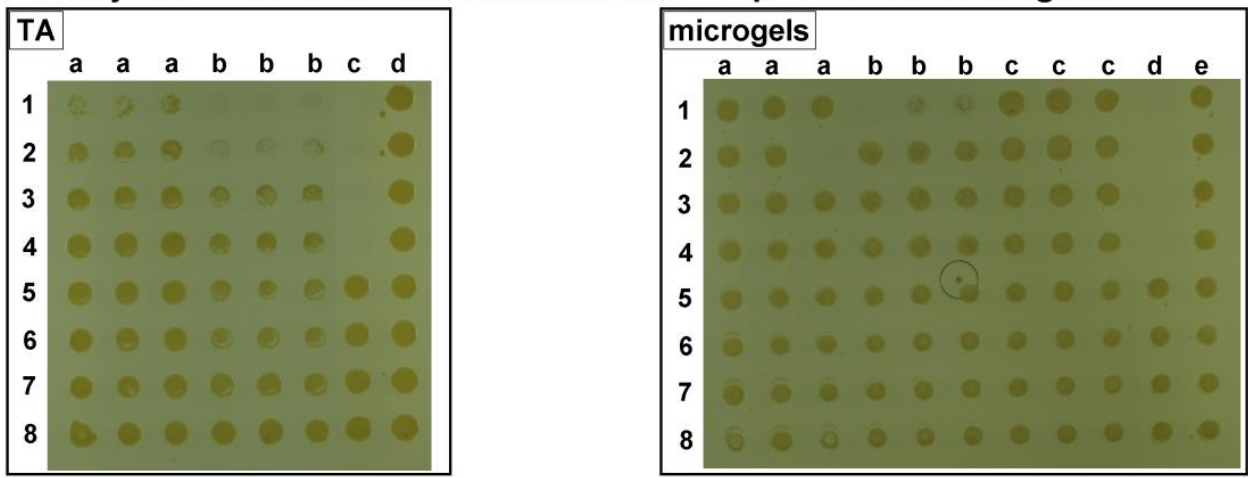

Figure 63. Minimum Bactericidal/Bacteriostatic Concentration test (MBC). a) Determination of the cell growth inhibition of X.Citri cells by REMA and polynomial regression and extrapolation of the obtained data. b) Characterization of the cell viability after the treatment with different compounds. Bacteria were exposed to the compounds at their IC $\mathrm{Co}_{90}$ for $24 \mathrm{~h}$, and then plated on solid NYG medium to test their viability. Each of the number lines represents the dose being diluted sequentially as performed in the REMA test. Pure TA (left): $a \rightarrow 100 \mu \mathrm{g} \cdot \mathrm{mL}^{-1}, \mathrm{~b} \rightarrow 1 \mathrm{mg} \cdot \mathrm{mL}^{-1}, \mathrm{c} \rightarrow$ positive control (Kanamycin, line 1-4)//vehicle control (water, line 5-8) and $\mathrm{d} \rightarrow$ negative control. Microgels (right): $\mathrm{a} \rightarrow \mathrm{VCL} / \mathrm{TA} 0.5 \mathrm{~mol} \%$ at $10 \mathrm{mg} \cdot \mathrm{mL}^{-1}, \mathrm{~b} \rightarrow \mathrm{VCL} / \mathrm{G} 10-10 \mathrm{~mol} \%$ at 10 $\mathrm{mg} \cdot \mathrm{mL}^{-1}, \mathrm{c} \rightarrow \mathrm{VCL} / \mathrm{TA} 0.5 \mathrm{~mol} \% / \mathrm{G} 10-10 \mathrm{~mol} \%$ at $10 \mathrm{mg} \cdot \mathrm{mL}^{-1}, \mathrm{~d} \rightarrow$ (Kanamycin, line 1-4)//vehicle control (water, line 5-8), $\mathrm{e} \rightarrow$ negative control. 
The results obtained for the different microgels point out that a high microgel concentration (above $5 \mathrm{mg} \cdot \mathrm{mL}^{-1}$ ) is necessary to reach an inhibiting cell growth effect. The inhibition effect observed at very high concentrations can be the consequence of a mechanical process due to an excess of material. Moreover, it is important to bear in mind that the incorporation of G10 is theoretically $10 \mathrm{~mol} \%$ of the microgel system, meaning that the antibacterial activity of the material correspond to a small fraction of the gel. In addition, the antibacterial effect of each substance was determined (Table 13).

Table 13. Antibacterial effect of TA and microgels.

\begin{tabular}{|c|c|c|}
\hline Compound & Bacterostatic effect & Bactericidal effect \\
\hline$T A$ & $0.1 \mathrm{mg} \cdot \mathrm{mL}^{-1}$ & $1 \mathrm{mg} \cdot \mathrm{mL}^{-1}$ \\
\hline VCL/TA-0.5mol\% & - & - \\
\hline$V C L / G 10-10 \mathrm{~mol} \%$ & $5 \mathrm{mg} \cdot \mathrm{mL}^{-1}$ & $10 \mathrm{mg} \cdot \mathrm{mL}^{-1}$ \\
\hline $\begin{array}{c}\text { VCL } / \text { TA-0.5mol\%/G10- } \\
10 \mathrm{~mol} \%\end{array}$ & $10 \mathrm{mg} \cdot \mathrm{mL}^{-1}$ & - \\
\hline
\end{tabular}

The cell viability was characterized after the treatment of the bacterial cells with different compounds. Thus, treated cells of the REMA were isolated and placed onto solid media plates (NYG-agar) without the studied compounds (TA and microgels). This experiment enables the evaluation of the ability of the cells to resume growth (Figure $63 \mathrm{~b})$. In the case of the microgels, the cell viability test reaffirms the absence of antibacterial effect for the PVCL/TA-microgel. However, it also indicates that the G10based microgels exhibit only a bacteriostatic effect and does not have bactericidal properties, meaning that the growth inhibition does not imply cell death.

Moreover, the different microgel systems were used to determine if they affect the bacterial cell division process by disruption of the Z-ring (divisome) in the cells. ${ }^{169}$ Previous studies have confirmed successfully that pure gallates exhibit this effect. However, the tested microgels PVCL/TA, PVCL/G10 and PVCL/TA/G10 did not show the same behavior. The stability of the FtsZ ring was visualized by fluorescent microscopy after labeling the divisome in the mutant strain X. citri amy::pGCD21-ZapA (this mutant expresses GFP-ZapA, an accessory cell division factor that interacts with the bacterial tubulin FtsZ, labeling indirectly the divisome). The obtained images corresponding to pure G10 and the microgel system VCL/G10-10mol\% are depicted in Figure 64. 


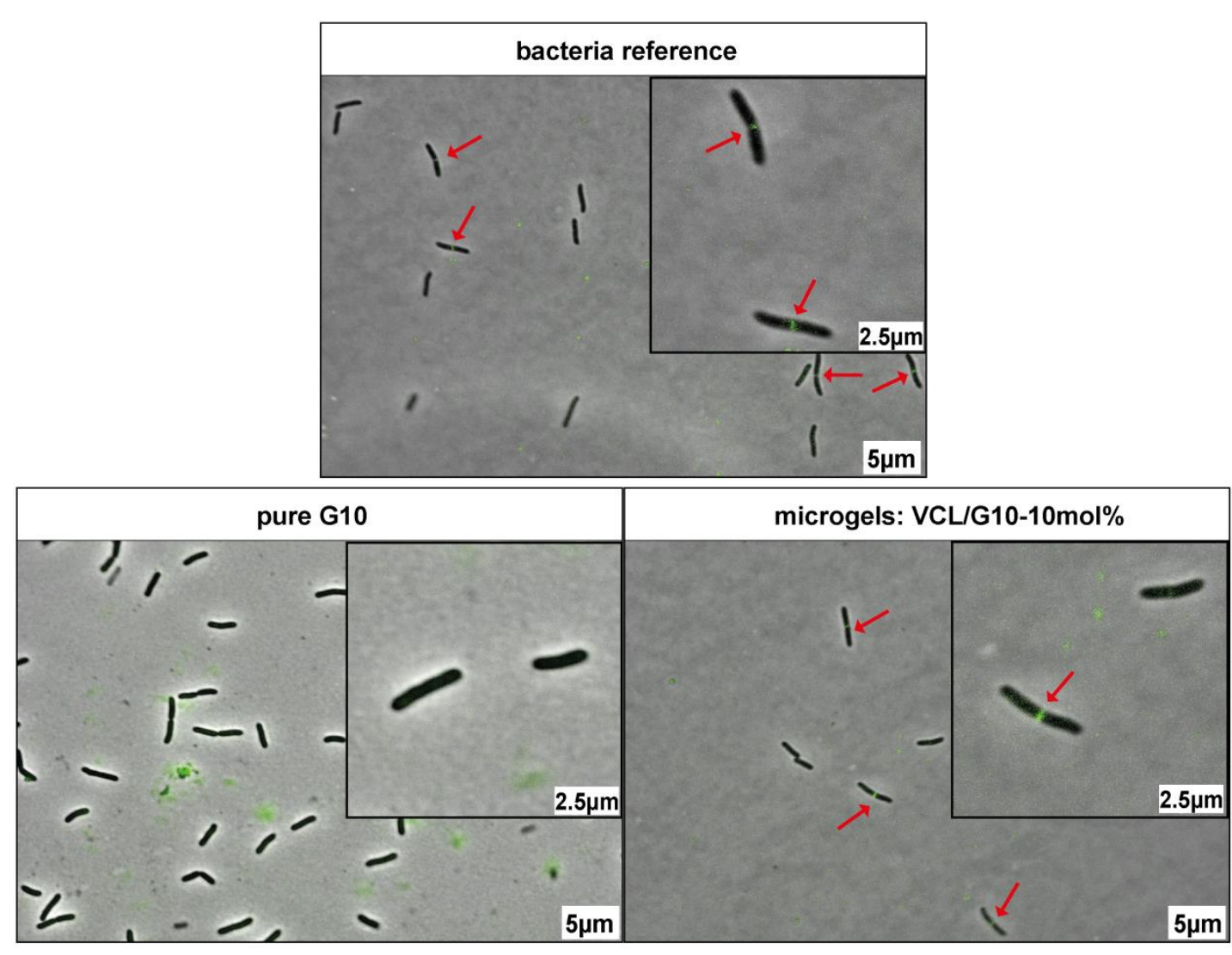

Figure 64. Microscopic study of the stability of the bacterial divisome after exposure to pure G10 (15 min contact) and PVCL/G10-microgels (30 min contact). The mutant X. citri amy::pGCD21-ZapA expressing GFPZapA shows the bacterial Z-ring (divisome) labeled in green. This was used to study the effects of the compounds on cell division.

In comparison to the bacteria reference, the protein ring disappears already after $15 \mathrm{~min}$ of bacterial contact with the pure gallate (G10). This confirms the ability of the gallate to inhibit the cell division process in X. Citri bacteria. However, no disruption was observed for the G10-based microgels. After 30 min contact, the FtsZ ring remains stable and can be seen as a bar positioned in the middle of the rod and perpendicular to the longer axis of the cells. Herein, it can be assumed that the physical interactions between the gallate molecules and the polymer chains (PVCL) are still very stable at the pH conditions of this test. It is probable that a small fraction of G10 is released and come into contact with the bacteria. Still, the major part remains entrapped within the polymer network and cannot be diffused trough the bacterial membrane to reach the protein septum. A complete delivery of the gallates is limited under the experimental conditions. The experiments should be carried out at a $\mathrm{pH} \geq 8.0$ to analyze the effect of the released gallates, since a basic medium would lead to the disintegration of the microgel network and release of the active compounds. 
Study of the stability of the bacterial cell membrane:
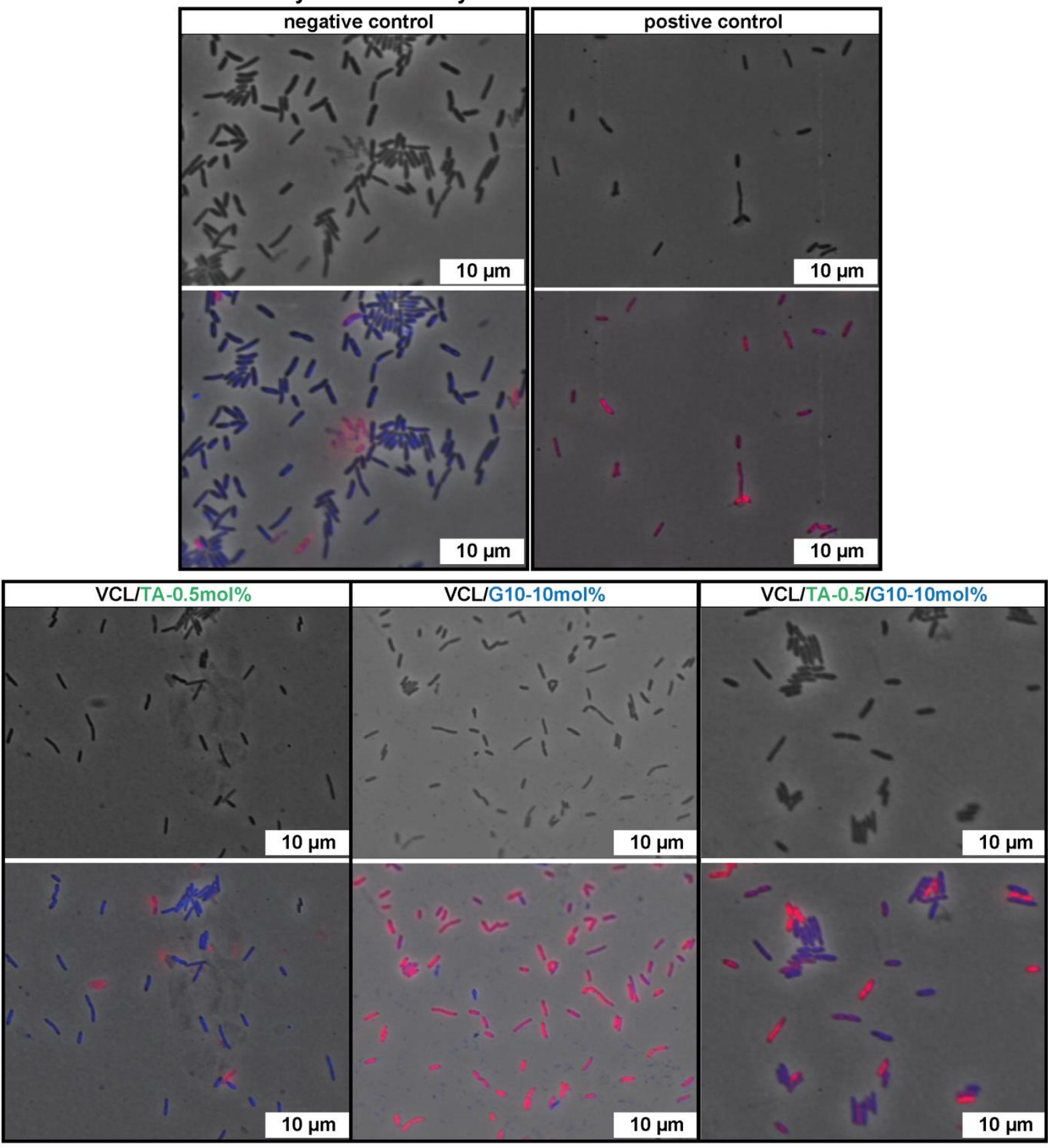

Figure 65. Characterization of stabibility of the the bacterial cell membrane in the presence of the different supramolecular microgels. Phase contrast (PhC, upper image) and Overlay of PhC with IP/DAPI fluorescence phase (bottom image).

The stability of the bacterial cell membrane in the presence of the different microgels was also analyzed by microscopy (Figure 65). Living cells are labeled in blue by the nucleic acid dye 4',6-diamidino-2-phenylindole (DAPI), which can penetrate intact membranes. Cells with corrupted membrane are labeled in red when treated with propidium iodide (IP), which only penetrated the cells with membrane corrupted. Only the microgel without G10 (VCL/TA-0.5 mol\%) does not show any effect. The presence of 
G10 within the microgel network (VCL/G10-10mol\% and VCL/TA-0.5 mol\%/G10$10 \mathrm{~mol} \%$ ) shows an influence on the membrane stability.

The analysis and its results suggest that the gallates remain stable and active although they are incorporated into the microgels. It could also indicate that small amounts are released under these experimental conditions and that these fractions come into contact with the bacteria. In addition, it could point out that the interaction between the microgel and the bacteria could be enough to permeabilize the cell membrane. An increase of the gallate content within the microgel network (up to $20 \mathrm{~mol} \%$ theo.) should improve the results.

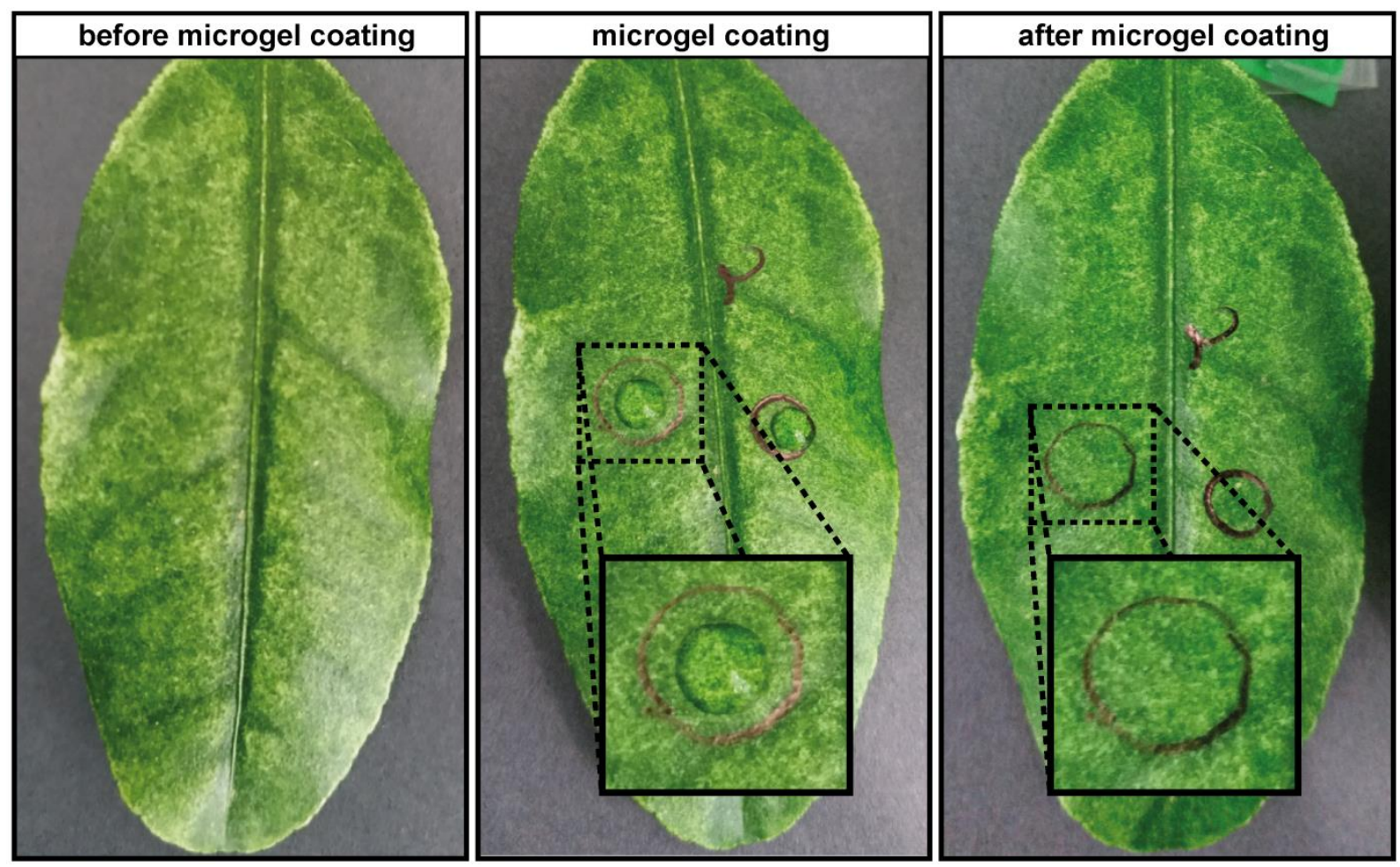

Figure 66. First process proposed for the coating of orange leaves using supramolecular microgels (VCL/TA$0.5 \mathrm{~mol} \% / \mathrm{G} 10-10 \mathrm{~mol} \%$ ) in a concentration of $\sim 0.1 \mathrm{mg} \cdot \mathrm{mL}^{-1}$.

In the end, proof-of-principal studies of leaf coating with the microgels were performed to finalize the presented $\mathrm{PhD}$ work. This part plays a decisive role for the future development of the project, since its main aim is to achieve a long-term and biocompatible approach that prevents the disease citrus canker during the agricultural production of citrus fruits. Therefore, the interaction of the materials with living plants is an essential research step. Figure 66 depicts the first procedure proposed for the coating of orange leaves with G10-based microgels. These experiments were performed 
by the author of this thesis at DWI-Leibniz-institute (Aachen-Germany). Herein, $10 \mu \mathrm{L}$ of an aqueous microgel solution $\left(\sim 0.1 \mathrm{mg} \cdot \mathrm{mL}^{-1}\right)$ was dropped on the leaf surface and left for drying at ambient conditions. As a preliminar observation, it can be affirmed possitively that the deposition of the microgels does not affect the optical properties of the plant. After the microgel solution is dried, there are no residues or color modifications of the leaf surface. This remark is important in view of production and product quality.

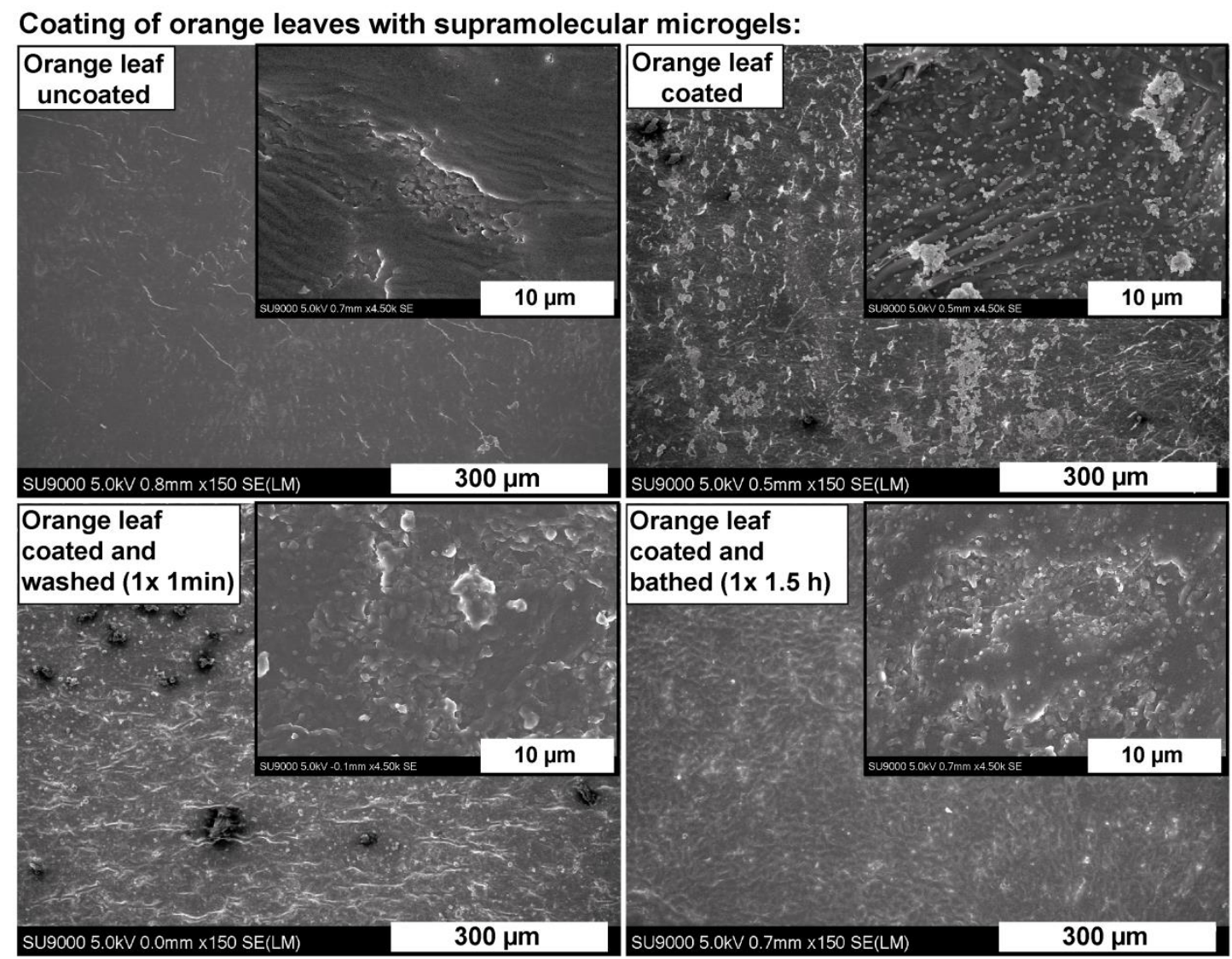

Figure 67. STEM characterization of the coating process on orange leaves.

Moreover, the leaf coating process was characterized by STEM (Figure 67). In this study, 4 different samples were characterized. First of all, an uncoated orange leaf was measured as reference for the experiments ("orange leaf uncoated", left, upper part). Its STEM-image reveals a slightly rough surface with minor porosities and uneven sections, but does not suggests any additional artifacts deposited on the surface. The second image shows the results for the coating process without whashing after drying ("orange leaf coated", right, upper part). In this case, one notices clearly the presence of the microgels on the leaf surface, even though they do not form a continous film. Higher 
concentrations of microgel should be studied to improve this issue. In comparison to this, 2 different samples were prepared to study the stability of the coating and analyze the influence of washing, since under natural conditions, the plants are exposed to rain seasons, which may wash the material from the leaf surfaces. Firstly, one coated sample was washed with water for up to one minute mimicking rain effect (dripping). The STEM results ("orange leaf coated and washed", left, bottom) exhibit that the plant surface remains modified and covered with external structures (microgels), but the microgels tend to form agreggates and show a slightly swollen state. Similar results are observed for the last sample, which was washed in a water bath for $1.5 \mathrm{~h}$ ("orange leaf coated and bathed", right, bottom).

Herein, the modification of the leaf structure can be noticed and one observes the presence of the spherical microgels on random regions of the leaf. Nevertheless, the preliminary tests showed positive results and can be used as a reference point for optimization steps. Furthermore, it is important to remark that these experiments were carried out before the modification of the microgels with the anchor peptides. This technology should improve the microgel attachment to the leaf surface and lead to very stable microgel coatings.

\subsection{Conclusions}

Stimuli-responsive and self-assembled nanomaterials are arousing great interest in design of novel drug delivery systems in view of specific and targeted release. In this section, possible applications for the supramolecular PVCL/TA-microgels were presented. The pH-triggered disassembly of these bio-based non-covalent microgels and their ability to complex metal ions offers great potential for their utilization as $\mathrm{pH}$ sensitive metal-carriers. In this chapter, it was confirmed that the PVCL/TA-microgels are capable for $\mathrm{Fe}^{3+}$-encapsulation and can serve as $\mathrm{pH}$-controlled Fe-delivery systems. Moreover, the incorporation of further phenolic compounds into PVCL-microgels could be performed using the optimized approach for the microgel synthesis. Herein, $(+)$-catechin was used as non-covalent crosslinker analogy to TA. The optimized PVCL/Cat-microgels showed a well-defined morphology, monodispersity and $\mathrm{pH}$ sensitivity. Lastly, the preliminary encapsulation of the hydrophobic antibacterial compound G10 into PVCL-microgels as single crosslinkers or co-crosslinkers in 
combination with TA was successful. In this case, the PVCL chains showed a stabilizing effect in aqueous solution during the microgels synthesis, which allowed the incorporation of G10 into the network core. The primary tests about the antibacterial properties of the supramolecular microgels are moreover especially important to highlight. The performed assays suggested in a preliminary form that the incorporation of the gallate G10 within the polymer network did not affect strongly its antibacterial properties. However, the entrapment of the active compound into the biocompatible polymeric container repressed its bactericidal effect. Finally, preliminary results for plant coating were presented. Herein, it could be showed that the microgels can attached onto the leaf surface without further functionalization, even though the coating achieved was inhomogeneous. The future modification of the microgels with the anchor peptide technology should help to improve the surface coating in a significant manner. In conclusion, the synthesis method reported in this thesis enables a facile process to produce supramoleculary assembled microgels, as well as a simple approach for the incorporation of bioactive hydrophobic components in a water-based system. 


\subsection{Supplementary Information}

a.) UV/Vis of SA/Fe-complexation:

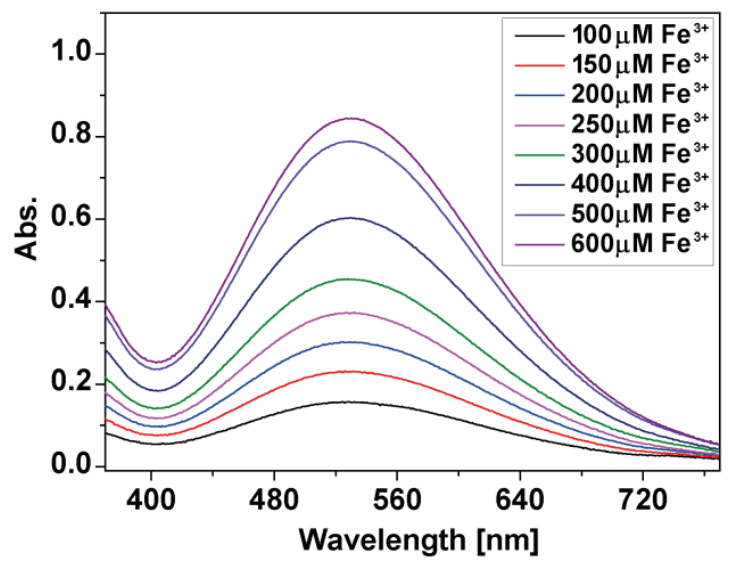

b.) Fe-content calibration:

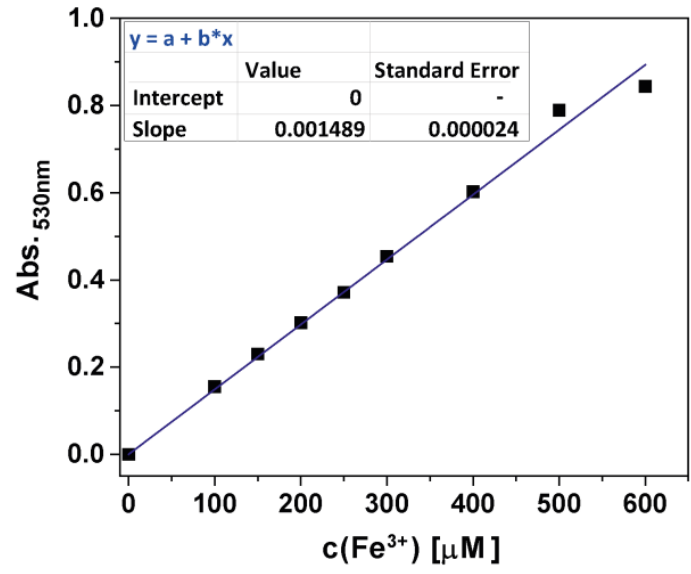

Figure 68. Calibration of the $\mathrm{Fe}^{3+}$-concentration by complexation with salicylic acid via UV/Vis-spectroscopy. 


\subsection{Experimental Section}

\subsubsection{Materials}

2,2'-Azobis-(2-methylpropionamidine) dihydrochloride (AMPA, 97\%, Aldrich), cetyltrimethylammonium bromide (CTAB, $\geq 97.0 \%$, Merck Millipore), tannic acid (TA, Sigma-Aldrich), sodium hydroxide solution (0.1 N, Sigma-Aldrich), $\mathrm{FeCl}_{3} \cdot 6 \mathrm{H}_{2} \mathrm{O}$ (reagent grade, $97 \%$, Sigma-Aldrich), salicylic acid ( $\geq 99.0 \%$, Sigma-Aldrich), ethanol, dopamine hydrochloride (Sigma-Aldrich), gallic acid ( $\geq 98 \%$, Sigma-Aldrich), (+)-catechin hydrate ( $\geq 98 \%$ (HPLC), powder, Sigma-Aldrich), and organic solvents ( $\mathrm{MeOH}, \mathrm{CHCl}_{3}, \mathrm{THF}, \mathrm{EtOH}$, DMSO), and dialysis membranes (Zellu Trans/Roth MWCO 3500 and 12000-14000, Carl Roth) were used without further treatment. $N$-vinylcaprolactam (VCL, $98 \%$, Aldrich) was distillated under vacuum before use. Deionized water was used for all experiments. $\mathrm{N}$-decyl gallate (G10) was synthesized, purified and characterized by the research group of Prof. Luis Regasini at the State University of São Paulo in São José do Rio Preto.

\subsubsection{Transport of $\mathrm{Fe}^{3+-i o n s}$}

\section{a) Loading of $\mathrm{Fe}^{3+-}$ Ions}

The incorporation of the $\mathrm{Fe}^{3+}$-ions into the supramolecular PVCL/TA-microgels was performed after the microgel synthesis and purification. Therefore, $50 \mu \mathrm{L}$ of a $\mathrm{FeCl}_{3}$ solution (500 mM) was added to $450 \mu \mathrm{L}$ of an aqueous microgel solution $\left(5 \mathrm{mg} \cdot \mathrm{mL}^{-1}\right.$ in dis. $\mathrm{H}_{2} \mathrm{O}, \mathrm{pH}: 6.3$ ) and stirred over night at room temperature. The product was cleaned via centrifugation and 3 -fold washing (dis. $\mathrm{H}_{2} \mathrm{O}$ ).

\section{b) Energy Dispersive X-Ray Spectroscopy}

The presence of Fe within the PVCL/TA-microgels was confirmed qualitatively by EDX (Oxford Instruments) in combination to STEM-measurements on an Ultra-high Resolution Scanning Electron Microscope SU9000 (Hitachi-High Technologies America, Inc.).This work was performed in part at the Center for Chemical Polymer Technology CPT, which is supported by the EU and the federal state of North Rhine-Westphalia (grant no. EFRE 3000883 02).

The quantification of the real $\mathrm{Fe}^{3+}$-content was determined indirectly by UV/Vis. The residual amount of $\mathrm{Fe}^{3+}$ from the loading process was calculated using salicylic acid as 
complexant agent. Therefore, $10 \mu \mathrm{L}$ of the supernatantwere added to $1 \mathrm{~mL}$ of a salicylic acid solution (5 mg. $\mathrm{mL}^{-1}$ in $\mathrm{EtOH} / \mathrm{H}_{2} \mathrm{O}: 1 / 1$ ) The measurements were performed on a Varian Cary 100 Bio UV / Vis Spectrophotometer and a Jasco V-780 VV Visible Spectrophotometer. All spectra were recorded at a wavelength between $\lambda=200 \mathrm{~nm}$ and $\lambda=800 \mathrm{~nm}$.

\section{c) pH-Triggered Release of $\mathrm{Fe}^{3+}$-Ions}

The loaded microgels were treated with an aqueous $\mathrm{HCl}$-solution (10 $\mathrm{mM}$ ) for the analysis of the $\mathrm{pH}$-controlled release of the iron ions. The microgel precipitate obtained after the loading procedure was dispersed in $500 \mu \mathrm{L}$ of the $\mathrm{HCl}$-solution (pH: 2.5) and stirred at room temperature. After different periods of time (1-13 days), the supernatant was withdrawn and fresh $\mathrm{HCl}$ solution was added. All supernatants were analyzed by $\mathrm{UV} / \mathrm{Vis}$ for the quantification of the $\mathrm{Fe}^{3+}$-amount.

\subsubsection{Incorporation of further phenolic compounds and Antibacterial Alkyl Gallates}

\section{a) Synthesis of VCL-Microgels using Further Phenols}

The incorporation of further phenolic derivatives was carried out analogy to the optimized synthesis of the PVCL/TA-microgels (route II) via precipitation polymerization in a semi batch procedure. The monomer VCL $\left(0,4 \mathrm{~g}, 139.2 \mathrm{~g} \cdot \mathrm{mol}^{-1}\right)$ was dissolved in $28 \mathrm{~mL} \mathrm{H}_{2} \mathrm{O}$ in a round glass flask (100 mL total volume). CTAB (1.2 mg, $364.45 \mathrm{~g} \cdot \mathrm{mol}^{-1}$ ) dissolved in dis. $\mathrm{H}_{2} \mathrm{O}$ was added to the monomer solution and the mixture was stirred 30 min under $\mathrm{N}_{2}$-atmosphere.The temperature was subsequently adjusted to $70{ }^{\circ} \mathrm{C}$, and the polymerization was started by the addition of AMPA $(10 \mathrm{mg}$, $271.19 \mathrm{~g} \cdot \mathrm{mol}^{-1}$ ). The phenolic compound (dopamine, gallic acid or (+)-catechin, G10, various amounts) was added (directly or dropwise) to the reaction after exactly $15 \mathrm{~min}$ of polymerization. The polymerization was stopped $1 \mathrm{~h}$ after the initiation. The product was purified by dialysis in deionized water for 4 days, and freeze dried.

\section{b) Synthesis of VCL-Microgels using Antibacterial Gallates}

Various VCL/TA/G10-microgels were synthesized by a semi-batch precipitation polymerization corresponding to the optimized synthesis of the PVCL/TA-microgels 
(route II). Therefore, stock solutions of TA and CTAB in deionized water, as well as G10 in DMSO were prepared. VCL $\left(0.4 \mathrm{~g}, 139.2 \mathrm{~g} \cdot \mathrm{mol}^{-1}\right)$ was dispersed in $28 \mathrm{~mL}$ deionized water and put into a round glass flask (100 mL total volume). After VCL was dissolved CTAB (1.2 mg, $364.45 \mathrm{~g} \cdot \mathrm{mol}^{-1}$ ) was added to the solution. The flask was closed with a septum and the solution was stirred and under $\mathrm{N}_{2}$ flow for $20 \mathrm{~min}$. Then, the reaction temperature was adjusted to $70{ }^{\circ} \mathrm{C}$. After heating up the solution, AMPA (10 mg, 271.19 $\left.\mathrm{g} \cdot \mathrm{mol}^{-1}\right)$ was added to the reaction mixture. TA $(0.0246 \mathrm{~g}, 0.48 \mathrm{~mL}, 0.5 \mathrm{~mol} \%)$ and G10 (variation of the amounts, $1 \mathrm{~mL}$ ) ) were added to the solution after exactly 15 minutes of the initiation. The addition of G10 was performed dropwise using a syringe pump. The microgel synthesis was carried out for $1 \mathrm{~h}$ in total. The products were purified by dialysis in deionized water and freeze dried.

\subsubsection{Characterization Methods}

\section{a) Yield Determination by Gravimetric Analysis}

Certain amount (1/30) of microgel solution was freeze dried after purification, and the mass of the product was calculated gravimetrically (triple determination). The yield values were determined from the quotient between the theoretical and real masses.

$$
\begin{aligned}
& \text { (4) } m_{\text {theo }}=\mathrm{m}_{\text {monomer }}+m_{\text {crosslinker }}+m_{\text {initiator }} \\
& \text { (5) } \bar{m}_{\text {real }}=\frac{\Sigma\left(m_{\text {product }} \cdot 30\right)}{3} \\
& \text { (6) Yield }=\frac{\bar{m}_{\text {real }}}{m_{\text {theo }}} \cdot 100 \%
\end{aligned}
$$

\section{b) Dynamic Light Scattering Measurements}

The microgel size/size distribution was characterized by DLS using ALV/CGS-3 Compact Goniometer System from the company ALV-GmbH with an ALV/LSE 5004 tau digital correlator and a JDS uniphase laser operating at $632.8 \mathrm{~nm}$. The scattering angle was fixed $\left(\theta=90^{\circ}\right)$ for all measurements. The temperature dependent experiments were performed in a range from 20 to $60^{\circ} \mathrm{C}$. All samples were filtered before the measurements with Chromafil@ Xtra syringe filters $(0.45$ or $1.20 \mu \mathrm{m})$. The results were obtained by ALV-Correlator Software V3.0. 


\section{c) Scanning Transmission Electron Microscopy}

The morphology of the microgels was studied by SEM and TEM on an Ultra-high Resolution Scanning Electron Microscope SU9000 (Hitachi-High Technologies America, Inc.). $100 \mu \mathrm{L}$ of each microgel solution $\left(0.1 \mathrm{~g} \cdot \mathrm{L}^{-1}\right)$ were diluted in $1 \mathrm{~mL}$ deionized water. $20 \mu \mathrm{L}$ of it were dropped on a TEM-grid (Carbon Film 200 Mesh Copper Grids, Electron Microscopy Sciences) and dried at room temperature overnight. The samples were sputtered with $2 \mathrm{~nm}$ carbon before the measurements.

This work was performed in part at the Center for Chemical Polymer Technology CPT, which is supported by the EU and the federal state of North Rhine-Westphalia (grant no. EFRE 3000883 02).

\section{d) Raman Spectroscopy Analysis}

The incorporation of the physical crosslinker TA in to the microgel network was confirmed and quantified by Raman spectroscopy on a Bruker RFS 100/S Raman spectrometer with a Nd:YAG laser (1064 nm). The measurements were carried out with a spectral resolution of $14 \mathrm{~cm}^{-1}$, power of $200 \mathrm{~mW}$ and 1000 scans. The results were analyzed using the software OPUS 4.0.

The quantification of the TA content within the microgels was performed using a calibration curve. Therefore, linear PVCL and TA were mixed in specific ratios and homogenized in methanol. The solvent was removed by evaporation at room temperature and the calibration samples were measured by Raman spectroscopy. The obtained results for the calibration and the corresponding graph are displayed in the next section.

\section{e) Study of Microgel Stability in Organic Solvents}

Microgel solutions (62.5 $\mathrm{mg} \cdot \mathrm{mL}^{-1}$ ) were prepared in $\mathrm{H}_{2} \mathrm{O}, \mathrm{THF}, \mathrm{CHCl}_{3}$ and $\mathrm{MeOH}$ and stirred overnight at room temperature. The stability of the various synthesized microgels in the organic solvents was investigated by STEM. 


\section{Summary and Outlook}

Supramolecular systems are considered promising in design of dynamic biomaterials with unique properties such as adaptability, responsiveness and recognition. In this view, not only the comprehension of biological mechanisms at the microscopic scale or atomic state is required, but also the incorporation of natural compounds as active agents is desired. In this thesis, the facile and novel design of supramolecular and stimuli-responsive microgels based on thermoresponsive PVCL and a multifunctional natural polyphenol is reported in detail, and initial results regarding their utilization as degradable carriers for the transport and targeted release of bioactive compounds are discussed.

The structure of this research was based on four primary sections. In the first part, the physical interactions between PVCL and TA were investigated introductorily. The proofof-principle study confirmed positively the rapid formation of stable PVCL/TAstructures in aqueous media. Due to its phenolic structure, tannic acid serves as a strong hydrogen donor and can interact with different substances by hydrogen bonding. Based on this, preliminary microgel syntheses were performed via radical precipitation polymerization using TA for the first time as a novel supramolecular and natural crosslinker. The polymerization of VCL in the presence of TA led to the successful formation of stable microgels. A systematical variation of the TA amount influenced the physical microgel properties such as size, polydispersity and surface charge. The crosslinking-degree could also be tuned, influencing the water uptake and the degree of swelling. A controlled increase of the TA content within the microgels guided to repression of the typical thermal responsiveness of PVCL-based materials.

Furthermore, the study of the microgel yield and monomer conversion during the first synthesis route indicated the degradation of the monomer VCL in the presence of TA. Herein, yields lower than $10 \%$ were obtained. In order to clarify these observations, the monomer stability was studied in detail. The results demonstrated that the monomer VCL is sensitive to hydrolysis under acidic conditions, but this susceptibility depends on the temperature and reaction time. At RT there was no effect of TA on the monomer structure, but at the polymerization temperature of $70^{\circ} \mathrm{C}$, the degradation of the monomer structure was observed. Still, the radical scavenge property of TA did not 
show any significant effect during the synthesis, due to the rapid polymerization of VCL. In conclusion, the first synthesis route can be utilized even for the production of welldefined supramolecular microgels with a high molar fraction of TA $(<11 \mathrm{~mol} \%)$.

In the second section, the optimization of the microgel synthesis in view of the yield was developed. A semi-batch process was applied to incorporate the crosslinker TA during the synthesis at different stages of the polymerization. Moreover, the addition type (direct or dropwise) was varied too in order to determine the best process parameters to increase the yields. A significant improvement of the microgel yield ( $>90 \%$ ) was achieved when the natural crosslinker TA was added at late stages of the polymerization. At this point, the most polymer chains are formed and stable so that the presence of TA has no negative effect during the microgel formation. Based on these results, TA was incorporated directly to the reaction after $15 \mathrm{~min}$ of polymerization to obtain the highest yield values.

After defining the parameters for the optimized synthesis route, the TA content was varied within the microgels and its influence on the microgel properties was determined. The increment of the TA amount led once more to a controlled decrease of the microgel size, as well as an increase of the crosslinking density. Additionally, the responsive property of the microgels could be adjusted one more time by changing the TA-content. The more TA within the polymeric network, the denser the microgels are and therefore, the less thermoresponsive they react. The microgels with higher TA fractions are highly crosslinked, therefore, the majority of the polymer functions are occupied interacting with the TA and cannot interact with the aqueous media. This fact hinders the swelling degree of the supramolecular PVCL-microgels.

The pH-sensitivity of the supramolecular PVCL/TA-microgels was analyzed in order to confirm the supramolecular formation of the microgels and also to obtain further information regarding their pH-triggered TA-release. The establishment of the analysis method was decisive for these experiments. The pH-triggered disassembly and simultaneous TA-release was studied preliminary by UV/Vis measurements, and the experiments were carried out in dependency of the $\mathrm{pH}$ and temperature. The results indicate positively that our physically crosslinked microgels can serve as pH-triggered TA-delivery systems. Under acidic or neutral conditions, the microgels remain stable. Contrary to this, at a pH of 11.7 almost $60 \%$ of TA was released after a time period of 15 
days. Still, it was confirmed successfully that the pH-induced disassembly can be repressed due to the thermoresponsiveness of the microgels.

Finally, the pH-triggered disassembly in combination with the complexation feature of the optimized PVCL/TA-microgels directs to their possible application as pH-sensitive carriers. In this thesis, it was concluded that the supramolecular microgels are able to transport $\mathrm{Fe}^{3+}$ in dependency of the environmental pH (loading degree up to $30 \%$ ). Additionally, the incorporation of further phenolic derivatives into PVCL-microgels could be achieved using the optimized approach for the microgel synthesis. Herein, (+)catechin could be utilized successfully as a physical crosslinker and the obtained PVCL/Cat-microgels showed a well-defined morphology, monodispersity and $\mathrm{pH}$ sensitivity. Lastly, the encapsulation of hydrophobic antibacterial alkyl gallates into PVCL/TA-microgels was performed successfully. All the results confirmed that the synthesis approach developed during this thesis offers a facile and innovative alternative to generate supramolecularly assembled microgels that can be used for the incorporation of metal ions and bioactive compounds. In conclusion, the PVCL/TAmicrogels developed within this scientific work present not only an innovative nanomaterial produced by a facile self-assembly method, but also enables the incorporation and preservation of different nature-based macromolecules and hydrophobic substances in a water-based system. The novel microgels are innovative nanomaterials that can be adapted to different requirements in the biotechnological field.

Due to the complexity of supramolecular systems and the broad variety of interesting properties of TA, there are still innumerable outstanding questions to work out in the future. A deeper-going investigation of the microgel structure at the molecular level by small angle neutron scattering (SANS) is required to determine the distribution of the crosslinker within the network. Moreover, the calculation of the binding constant between VCL as well as PVCL and TA should be optimized by ITC. This study would provide detailed information about the stability of the system and its $\mathrm{pH}$-sensitivity. The further optimization of the presented applications should be carried out as well. Herein, the utilization of other metals or the combination of further bioactive compounds would be interesting for the production of multifunctional biomaterials. In terms of specific 
bio-applications, studies in view of biocompatibility, toxicity and antibacterial and antioxidant properties among others are essential. 


\section{ACKNOWLEDGMENTS}

First and foremost, I would like to thank Prof. Dr. Andrij Pich for the great opportunity to develop my doctoral work in his research group under his supervision. I am very thankful for the interesting topic, his constant support and trust, as well as his always helpful advices during this research. In addition, I thank Prof. Dr. Walter Richtering for the examination of my $\mathrm{PhD}$ thesis as second reviewer.

I would also like to express my gratitude to my research colleagues at the institute. Herein, I thank all group members and technicians for the friendly recommendations and help throughout my PhD studies. A special thanks goes to Sabrina Mallmann, who executed the STEM measurements at the Center for Chemical Polymer Technology CPT (work supported by the EU and the federal state of North Rhine-Westphalia (grant no. EFRE 3000883 02)). I am also grateful to Dr. Walter Tillmann for the Raman experiments, Rainer Haas for the GPC analysis and Claudia Pörschke for the TGA and DSC measurements.

Moreover, I thank Alexander Töpel for the AFM characterization, Michael Kather for the calorimetric anaylsis and Chaolei Hu for the synthesis of the linear PVCL. Likewise, it is a pleasure to thank my research students Gil van Wissen and Hülya Güven for their wellperformed work in the laboratory and the final contributions to my $\mathrm{PhD}$ results.

To my project partners in Brazil; Prof. Henrique Ferreira and Guillermi Dilarri, all my gratitude for the great team work, the performance of the antibacterial studies, the interesting scientific discussions and the revisions of the chapter 5.2.3. of my thesis. This work section was financially supported by the Federal Ministry of Education and Research - BMBF and the São Paulo Research Foundation - FAPESP.

In addition, I would like to thank to all persons that supported me along the way and made the time of my PhD unforgettable. I especially thank Ayse Deniz and Wenjing Xu for their unconditional friendship and the great moments we spent together at the institute. My biggest thanks go to my parents Astrid López and Néstor Molano, and my brother Néstor Francisco Molano López, for always being there for me and encouraging me to achieve my goals.

Finally, my endless gratitude to my husband Jens Artz: thank you for your helpful advices during our scientific conversations, as well as for the revision of my thesis, but in particular I thank you for your great company and for motivating me every day. 


\section{LIST OF ABBREVIATIONS}

\begin{tabular}{ll}
${ }^{1} \mathrm{H}-\mathrm{NMR}$ & Proton Nuclear Magnetic Resonance \\
Abs & Absorbance \\
AFM & Atomic Force Microscopy \\
BIS & $N, N$-Methylenebis(Acrylamide) \\
${ }^{\circ} \mathrm{C}$ & Celcius \\
$\mathrm{c}$ & Concentration \\
Cat & (+)-Catechin \\
cm & Centimeter \\
CTAB & Cetrimonium Bromide \\
DAPI & $4^{\prime}, 6$-Diamidino-2-Phenylindole \\
dis. & Distilled \\
DLS & Dynamic Light Scattering \\
DMF & Dimethylformamide \\
DMSO & Dimethyl Sulfoxide \\
DNA & Deoxyribonucleic Acid \\
DSC & Differential Scanning Calorimetry \\
EA & Ethyl Acrylate \\
EDX & Energy Dispersive X-Ray Spectroscopy \\
EtOH & Ethanol \\
FBGCs & Foreign Body Giant Cells \\
G10 & N-Decyl Gallate \\
GA & Gallic Acid \\
GFP & Green Fluorescent Protein \\
GPC & Gel Permeation Chromatography \\
h & hour \\
HEMA & 2-Hydroxyethylmethacrylate \\
IP & Propidium Iodide \\
ITC & Isothermal Titration Calorimetry \\
$\mathrm{K}_{\alpha}$ & Siegbahn notation - emission line \\
$\mathrm{K}_{a}$ & Association Constant \\
$\mathrm{K}_{\mathrm{d}}$ & Dissociation Constant \\
LCST & Lower Critical Solution Temperature \\
& \\
\hline
\end{tabular}




\begin{tabular}{|c|c|}
\hline MAA & Methacrylic Acid \\
\hline $\mathrm{MeOH}$ & Methanol \\
\hline $\mathrm{mg}$ & Milligram \\
\hline MIC & Minimum Inhibitory Concentration \\
\hline $\min$ & Minute \\
\hline$\mu \mathrm{L}$ & Microliter \\
\hline $\mathrm{mL}$ & Milliliter \\
\hline$\mu \mathrm{g}$ & Microgram \\
\hline $\mathrm{mg}$ & Milligram \\
\hline$\mu \mathrm{Gel}$ & Microgel \\
\hline MMA & Methyl Methacrylate \\
\hline $\mathrm{M}_{\mathrm{n}}$ & Number Average Molecular Weight \\
\hline Mob & Electrophoretic Mobility \\
\hline $\mathrm{mol} \%$ & Mole percentage \\
\hline $\mathrm{n}$ & Binding Stoichiometry \\
\hline NYG & Nutrient/Yeast extract/Agar \\
\hline NIPAAm & $N$-Isopropylacrylamide \\
\hline PCL & Polycaprolactone \\
\hline PDI & Polydispersitiy Index \\
\hline PEO-b-PCL & Poly(Ethylene Oxide)-B-Poly(E-Caprolactone) \\
\hline $\mathrm{PhC}$ & Phase Contrast \\
\hline PHEMA & Poly(2-Hydroxyethylmethacrylate) \\
\hline PLA & Polylactic Acid \\
\hline PLGA & Poly(Lactic-co-Glycolic Acid) \\
\hline PMMA & Poly(Methyl Methacrylate) \\
\hline PVCL & Poly(N-Vinylcaprolactam) \\
\hline RAFT & Reversible Addition-Fragmentation Chain Transfer \\
\hline REMA & Resazurin Microtiter Assay \\
\hline $\mathrm{R}_{\mathrm{h}}$ & Hydrodynamic Radius \\
\hline RNA & Ribonucleic Acid \\
\hline RT & Room Temperature \\
\hline siRNA & Short Interfering Ribonucleic Acid \\
\hline STEM & Scanning Transmission Electron Microscopy \\
\hline $\mathrm{T}$ & Temperature \\
\hline
\end{tabular}




$\begin{array}{ll}\mathrm{t} & \text { time } \\ \text { TA } & \text { Tannic Acid } \\ \text { TGA } & \text { Thermal Gravimetric Analysis } \\ \text { theo. } & \text { Theoretical } \\ \text { THF } & \text { Tetrahydrofuran } \\ \text { UV/Vis } & \text { Ultraviolet-Visible SPECTROSCOPY } \\ \text { VCL } & \text { N-Vinylcaprolactam } \\ \text { VPTT } & \text { Volume Phase Transition Temperature } \\ \text { WBSSH } & \text { White-Bate-Smith-Swain-Haslam } \\ \text { X. Citri } & \text { Xanthomonas Citri } \\ \text { XPS } & \text { X-ray photoelectron spectroscopy } \\ \Delta G & \text { Gibbs energy } \\ \Delta H & \text { binding enthalpy } \\ \Delta S & \text { entropy } \\ \% & \text { percentage }\end{array}$




\section{LIST OF FIGURES}

FIGURE 1. GRAPHICAL REPRESENTATION OF THE MICROGELS RESPONSE TO EXTERNAL STIMULI.........................................-9 -

FIGURE 2. SCHEMATIC REPRESENTATION OF VARIOUS NON-COVALENT INTERACTIONS. .....................................................- 11 -

FigURE 3. CHEMICAL STRUCTURE OF THE NATURAL POLYPHENOLIC COMPOUND TA...................................................... 14 -

FIGURE 4. SCOPE OF THE THESIS: SYNTHESIS OF BIO-INSPIRED SUPRAMOLECULAR PVCL/TA-MICROGELS AND THEIR UTILIZATION AS DEGRADABLE AND STIMULI-RESPONSIVE CARRIERS FOR BIOACTIVE COMPOUNDS......................................- 16 -

FiguRE 5. PRIOR TEST TO DETERMINE THE FORMATION OF BONDS BETWEEN PVCL LINEAR CHAINS AND TANNIC ACID. . - 19 FigURE 6. PRELIMINARY THERMODYNAMIC CHARACTERIZATION OF THE INTERACTIONS BETWEEN LINEAR PVCL (M $60 \mathrm{KG} \cdot \mathrm{MOL}^{-1}$ ) AND TA VIA ITC. $-20-$

FIGURE 7. FORMATION OF MICROGEL/METAL ION-BASED HYDROGELS …........................................................................ 23 -

FIGURE 8. SCHEME OF THE MICROGEL SYNTHESIS..................................................................................................... 24

FigURE 9. IN-SITU DLS MEASUREMENT OF $\mathrm{R}_{\mathrm{H}}$ DURING THE POLYMERIZATION (SAMPLE: VCL/TA-0.5MOL-\%) AND CORRESPONDING 1.A.-B.) TEM AND SEM IMAGES. .................................................................................................. 25 FIGURE 10. A) REFERENCE: SYNTHESIS OF PVCL/TA-MICROGELS CARRIED OUT AS DESCRIBED PREVIOUSLY. B) EFFECT OF THE TEMPERATURE: MODIFICATION OF THE SECOND SYNTHESIS STEP. C) EFFECT OF THE SUFACTANT: MICROGEL SYNTHESIS WITHOUT SURFACTANT. $-26-$

FiguRE 11. STUdy OF THE CHEMICAL COMPOSITION OF THE PVCL/TA-MICROGELS BY RAMAN SPECTROSCOPY.............. 27 FIGURE 12. SEM IMAGES OF PVCL/TA-MICROGELS WITH VARIOUS TA-AMOUNTS. ...................................................... 30 FIGURE 13. PARTICLE SIZE, PDI VALUES AND B.) ELECTROPHORETIC MOBILITY AT $20^{\circ} \mathrm{C}$ AND PH 6.5 IN DEPENDENCE OF THE TA-CONTENT $31-$

FIGURE 14. SCHEMATIC REPRESENTATION OF THE THERMORESPONSIVE BEHAVIOR OF PVCL-BASED MICROGELS AND HYDRODYNAMIC RADII OF THE VARIOUS PVCL/TA MICROGELS IN DEPENDENCY OF TEMPERATURE MEASURED BY DLS. - 31 FIGURE 15. ILLUSTRATION OF THE PH SENSITIVITY, SEM-IMAGES OF THE MICROGELS AT DIFFERENT PH VALUES, AS WELL AS TITRATION AND TURBIDITY CURVES MEASURED BY ADDING NAOH (0.1M) (VCL/TA 0.5 MOL TEMPERATURE $-33-$

FIGURE 16. RAMAN CALIBRATION FOR THE QUANTIFICATION OF THE REAL TA-CONTENT. $36-$

FigURE 17. INITIAL STUDY OF THE MOLECULAR WEIGHT VIA GPC (IN DMF). MOLECULAR MASS DISTRIBUTION OF A.) PVCL CHAINS CONTAINED IN THE MICROGELS (VCL/TA-0.5MOL\% SAMPLE AFTER THE PH DEGRADATION) AND B.) PVCL CHAINS SYNTHESIZED BY PRECIPITATION POLYMERIZATION WITHOUT CROSSLINKER.................................................................. 37 .

FIGURE 18. ILLUSTRATED MICROGEL FORMATION BASED ON H-BONDING BETWEEN TANNIC ACID AND PVCL................... 43 FIGURE 19. A) YIELDS OF THE PVCL/TA-MICROGELS IN DEPENDENCY OF THE CROSSLINKER CONTENT AND B) ${ }^{1} \mathrm{H}-\mathrm{NMR}$ STUDY OF THE STABILITY OF VCL IN THE PRESENCE OF TA AT RT. C) ${ }^{1}$ H-NMR SPECTRUM OF TA. $44-$ FIGURE 20. A) ${ }^{1}$ H-NMR-STUDY OF THE STABILITY OF VCL IN THE PRESENCE OF TA AND B) SCHEMATIC REPRESENTATION OF THE VCL HYDROLYSIS IN THE PRESENCE OF TA AT THE POLYMERIZATION TEMPERATURE $\left(70{ }^{\circ} \mathrm{C}\right)$............................... 46 FIGURE 21. SCHEME FOR THE OPTIMIZED REACTION SYNTHESIS OF THE PVCL/TA-MICROGELS. $-47-$ FigURE 22. OPTIMIZATION OF THE MICROGEL SYNTHESIS. A) YIELDS IN DEPENDENCY OF THE TYPE OF TA-ADDITION AND WAITING TIME. B) EFFECT OF THE TIME BETWEEN INITIATION AND TA-ADDITION ON THE MICROGEL MORPHOLOGY.......- 48 FIGURE 23. STUDY OF THE MORPHOLOGY AND DIMENSION OF THE OPTIMIZED PVCL/TA-MICROGELS IN DEPENDENCY OF THE TA-CONTENT. A) PARTICLE SIZE, PDI VALUES AS WELL AS C) STEM IMAGES. 
FIGURE 25. INFLUENCE OF THE SURFACTANT AMOUNT USED DURING THE MICROGEL SYNTHESIS.. -50 -

FIGURE 26. RAMAN MEASUREMENTS FOR THE ANALYSIS OF THE CHEMICAL COMPOSITION OF THE PVCL/TA-MICROGELS. ....51 -

FiguRE 27. CHARACTERIZATION OF THE THERMAL DEGRADATION OF PVCL/TA-MICROGELS BY TGA. $-52-$

FIGURE 28. STABILITY OF PVCL/TA-MICROGELS IN DIFFERENT SOLVENTS -. 54 -

FIGURE 29. STUDY OF THE MICROGEL ELECTROPHORESIS $55-$

FIGURE 30. A) DLS-STUDY OF THE THERMORESPONSIVENESS OF THE OPTIMIZED PVCL/TA-MICROGELS AND B) COMPLEMENTARY ANALYSIS OF THE WATER UP-TAKE CAPACITY VIA DSC. - 55 -

FIGURE 31. INFLUENCE OF THE MEDIUM PH ON THE STABILITY OF THE OPTIMIZED PVCL/TA-MICROGELS.. $56-$ FIGURE 32. ILLUSTRATED SYNTHESIS ROUTE OF THE MICROGEL PRODUCTION FROM LINEAR PVCL CHAINS WITH SPECIFIC MOLECULAR WEIGHTS. $-57-$

FIGURE 33. STEM IMAGES OF SUPRAMOLECULAR PRODUCTS OBTAINED BY THE SYNTHESIS OF MICROGELS USING DEFINED PVCL CHAINS AS PRECURSORS. $58-$

FigURE 34. SCHEMATIC REPRESENTATION OF THE PH-INDUCED DISINTEGRATION OF THE SUPRAMOLECULAR PVCL/TAMICROGELS. $67-$

FIGURE 35. UV/VIS CHARACTERIZATION OF THE TA-STRUCTURE IN DEPENDENCY OF THE PH, AND SELECTION MEDIUM FOR THE PH TREATMENT UNDER BASIC CONDITIONS. - 69 -

FIGURE 36. ILLUSTRATION OF VARIOUS SET-UPS USED FOR THE PH-TRIGGERED DISASSEMBLY EXPERIMENTS. ..................- 70 FIGURE 37. COMPARISON OF THE USED SET-UPS IN VIEW OF SEPARATION OF THE PVCL-CHAINS FROM TA......................- 70 FIGURE 38. PH-TRIGGERED DISASSEMBLY OF VCL/TA-2MOL\% \%нE.-MICROGELS (OPTIMIZED SYNTHESIS, $\left.\mathrm{TA}_{\text {REAL }}: 1.29 \mathrm{MOL} \%\right)$ AND SIMULTANEOUS TA-RELEASE. $71-$ FIGURE 39. PVCL/TA-MICROGELS AFTER PH-TRIGGERED DISASSEMBLY AT DIFFERENT PH VALUES AND TEMPERATURES.....72 -

FIGURE 40. PH-TRIGGERED DISASSEMBLY OF SUPRAMOLECULAR MICROGELS WITH DIFFERENT TA-CONTENT

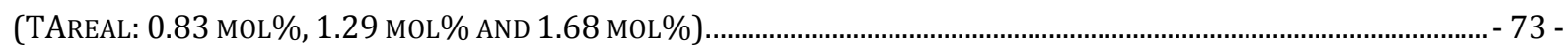

FIgURE 41. CALIBRATION BY UV/VIS OF THE TA-CONCENTRATION AT DIFFERENT PH VALUES.......................................- 76 FIgURE 42. . COMPARISON OF A) UV/VIS SPECTRA OF PVCL AND TA AT PH 11.7, AS WELL AS B) DIALYSIS MEMBRANES

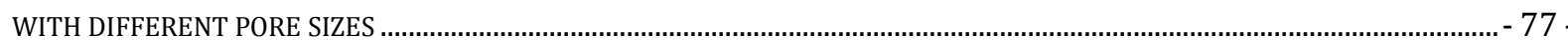

FIGURE 43. . UV/VIS SPECTRA OF PH-TRIGGERED DISASSEMBLY EXPERIMENTS CARRIED OUT AT DIFFERENT PH VALUES. - 77

FIGURE 44. UV/VIS SPECTRA OF PH-TRIGGERED DISASSEMBLY EXPERIMENTS PERFORMED ON PVCL/TA-MICROGELS WITH VARIOUS TA-MOLAR FRACTIONS. .............................................................................................................................. 78

FIGURE 45. SUPRAMOLECULAR MICROGELS AS PH-SENSITIVE FE ${ }^{3+}$-CARRIERS................................................................ 84 -

FIGURE 46. INCORPORATION OF FE3+ WITHIN THE PVCL/TA-MICROGELS. ....................................................................... 85 -

FigURE 47. ELEMENTAL ANALYSIS OF FE ${ }^{3+}$-LOADED PVCL/TA-MICROGELS (TA: 1.29 MOL\% ). LEFT: STEM-IMAGE WITH ELEMENTAL MAPPING. RIGHT: EDX-SPECTRUM...................................................................................................... 86

FIGURE 48. PH-CONTROLLED RELEASE OF FE ${ }^{3+}$ FROM PVCL/TA-MICROGELS. ................................................................. 87 -

FIGURE 49. PHENOLIC DERIVATIVES AS CROSSLINKERS FOR THE SYNTHESIS OF SUPRAMOLECULAR MICROGELS.................- 88 - 
FIGURE 50. SYNTHESIS ROUTE FOR THE INCORPORATION OF VARIOUS PHENOLIC DERIVATIVES INTO MICROGELS AS

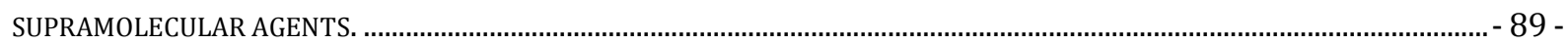

FIGURE 51. NON-COVALENT COMPOSITE PRODUCED BY INTERACTIONS BETWEEN (+)-CATECHIN AND PVCL. ................. - 91 FIGURE 52. PRODUCTION OF SUPRAMOLECULAR MICROGELS BASED ON (+)-CATECHIN AS PHYSICAL CROSSLINKER..........- 91 FIGURE 53. DETERMINATION BY RAMAN SPECTROSCOPY OF THE INCORPORATION OF (+)-CATECHIN AS PHYSICAL

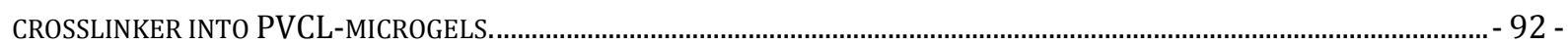
FIGURE 54. SYSTEMATIC VARIATION OF THE (+)-CATECHIN-CONTENT DURING THE MICROGEL SYNTHESIS......................- 93 FIGURE 55. . PH-STABILITY OF THE SUPRAMOLECULAR PVCL/CAT-MICROGELS. ............................................................ 94 FIGURE 56. GRAPHIC ILLUSTRATION OF THE PROPLANTA PROJECT............................................................................... 95 FIGURE 57. INCORPORATION OF G10 AS NON-COVALENT (CO-) CROSSLINKER. LEFT AND MIDDLE: MICROGELS CONTAINING G10 AS SINGLE CROSSLINKER. RIGHT: MICROGELS CONTAINING A COMBINATION OF TA AND G10 AS MULTIPLE CROSSLINKERS - 97 -

FIGURE 58. STUDY OF THE CHEMICAL COMPOSITION OF G10-BASED MICROGELS BY RAMAN SPECTROSCOPY....................- 98 FIGURE 59. OPTIMIZATION OF THE GALLATE G10 (10 MOL\%) ENCAPSULATION INTO SUPRAMOLECULAR MICROGELS. ..- 99 FIGURE 60. TIME VARIATION FOR G10-ADDITION DURING THE MICROGEL SYNTHESIS USING G10 (2 MOL\%) AS A COCROSSLINKER IN COMBINATION WITH TA (0.5 MOL\%) ............................................................................................... - 100 FIGURE 61. VARIATION OF G10-AMOUNT WITHIN PVCL/TA-MICROGELS. ................................................................... - 101 FIGURE 62. STABILITY OF G10-BASED MICROGELS IN ORGANIC SOLVENTS. $-102-$ FigURE 63. Minimum BACTERICIDAL/BACTERIOSTATIC CONCENTRATION TEST (MBC). A) DETERMINATION OF THE CELL GROWTH INHIBITION OF X.CITRI CELLS BY REMA AND POLYNOMIAL REGRESSION AND EXTRAPOLATION OF THE OBTAINED DATA. B) CHARACTERIZATION OF THE CELL VIABILITY AFTER THE TREATMENT WITH DIFFERENT COMPOUNDS. BACTERIA WERE EXPOSED TO THE COMPOUNDS AT THEIR IC 90 FOR 24 H, AND THEN PLATED ON SOLID NYG MEDIUM TO TEST THEIR VIABILITY. EACH OF THE NUMBER LINES REPRESENTS THE DOSE BEING DILUTED SEQUENTIALLY AS PERFORMED IN THE REMA TEST. PURE TA (LEFT): A $\rightarrow 100 \mu \mathrm{G}^{\cdot} \mathrm{ML}^{-1}, \mathrm{~B} \rightarrow 1 \mathrm{MG} \cdot \mathrm{ML}^{-1}, \mathrm{C} \rightarrow$ POSITIVE CONTROL (KANAMYCIN, LINE 14)//VEHICLE CONTROL (WATER, LINE 5-8) AND D $\rightarrow$ NEGATIVE CONTROL. MiCROGELS (RIGHT): A $\rightarrow$ VCL/TA 0.5MOL\% AT $10 \mathrm{MG} \cdot \mathrm{ML}^{-1}, \mathrm{~B} \rightarrow \mathrm{VCL} / \mathrm{G} 10-10 \mathrm{MOL} \%$ AT $10 \mathrm{MG} \cdot \mathrm{ML}^{-1}, \mathrm{C} \rightarrow \mathrm{VCL} / \mathrm{TA} 0.5 \mathrm{MOL} \% / \mathrm{G} 10-10 \mathrm{MOL} \%$ AT $10 \mathrm{MG}^{*} \mathrm{ML}^{-1}, \mathrm{D} \rightarrow$ (KANAMYCIN, LINE 1-4)//VEHICLE CONTROL (WATER, LINE 5-8), E $\rightarrow$ NEGATIVE CONTROL $-103-$ FIGURE 64. MICROSCOPIC STUDY OF THE STABILITY OF THE BACTERIAL DIVISOME AFTER EXPOSURE TO PURE G10 (15 MIN CONTACT) AND PVCL/G10-MICROGELS (30 MIN CONTACT). THE MUTANT X. CITRI AMY::PGCD21-ZAPA EXPRESSING GFPZAPA SHOWS THE BACTERIAL Z-RING (DIVISOME) LABELED IN GREEN. THIS WAS USED TO STUDY THE EFFECTS OF THE COMPOUNDS ON CELL DIVISION $-105-$ FIGURE 65. CHARACTERIZATION OF STABIBILITY OF THE THE BACTERIAL CELL MEMBRANE IN THE PRESENCE OF THE DIFFERENT SUPRAMOLECUlar MiCROGELS. PhaSE CONTRAST (PhC, UPPER IMAGE) AND OVERLAY OF PhC WITH IP/DAPI FLUORESCENCE PHASE (BOTTOM IMAGE). - 106 FIGURE 66. FIRST PROCESS PROPOSED FOR THE COATING OF ORANGE LEAVES USING SUPRAMOLECULAR MICROGELS (VCL/TA-0.5MOL\%/G10-10MOL\%) IN A CONCENTRATION OF $\sim 0.1 \mathrm{MG} \cdot \mathrm{ML}^{-1}$ - 107 FIGURE 67. STEM CHARACTERIZATION OF THE COATING PROCESS ON ORANGE LEAVES. $-108-$ FIgURE 68. CALIBRATION OF THE FE ${ }^{3+}$-CONCENTRATION BY COMPLEXATION WITH SALICYLIC ACID VIA UV/VISSPECTROSCOPY $-111-$ 


\section{LIST OF TABLES}

TABLE 1. THERMODYNAMIC PARAMETERS OBTAINED BY ITC.......................................................................................... - 22 -

TABLE 2. THEORETICAL AND EXPERIMENTALLY DETERMINED TA AMOUNTS IN MICROGELS................................................ - 28 -

TABLE 3. CALCULATED VALUES FOR THE CALIBRATION CURVE....................................................................................... 36 -

TABLE 4. QUANTIFICATION OF THE REAL TA-CONTENT WITHIN THE PVCL/TA-MICROGELS................................................- 37 -

TABLE 5. QUANTITIES OF TA USED FOR THE MICROGEL SYNTHESIS, HYDRODYNAMIC RADII AND PDI OF THE PVCL/TA-

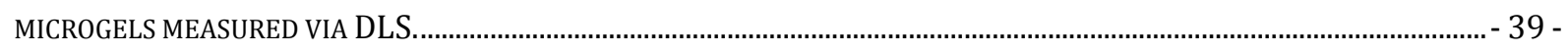

TABLE 6. REAL TA CONTENT OF THE SUPRAMOLECULAR MICROGELS QUANTIFIED BY RAMAN. ............................................ - 52 -

TABLE 7. DEGRADATION TEMPERATURES OF VCL-MONOMER AND TA DETERMINED BY TGA............................................... - 53 -

TABLE 8. HYDRODYNAMIC RADII, PDIS AND YIELDS OF THE OPTIMIZED PVCL/TA-MICROGELS.......................................... - 61 -

TABLE 9. Volumes USED FOR UV/VIS-CALIBRATION OF TA AT DIFFERENT PH-VALUES..................................................... - 80 -

TABLE 10. FORMATION OF SUPRAMOLECULAR STRUCTURES BY PHYSICAL INTERACTIONS BETWEEN PVCL AND PHENOLIC

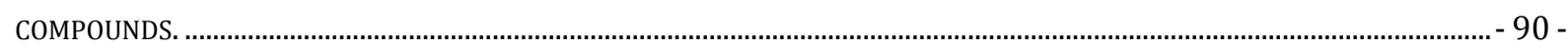

TABLE 11. HYDRODYNAMIC RADII AND PDI VALUES CALCULATED BY DLS AND GRAVIMETRIC YIELD OF THE FIRST GALLATE-

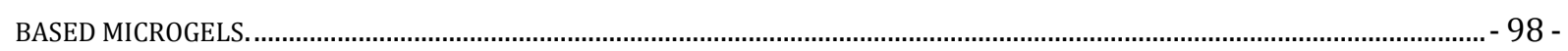

TABLE 12. MIC OF PURE TA AND MICROGELS CALCULATED BY REMA. …….................................................................. - 102 -

TABLE 13. ANTIBACTERIAL EFFECT OF TA AND MICROGELS. .......................................................................................... - 104 - 


\section{REFERENCES}

1. Warren, S. C.; Guney-Altay, O.; Grzybowski, B. A., Responsive and Nonequilibrium Nanomaterials. The Journal of Physical Chemistry Letters 2012, 3 (15), 2103-2111.

2. He, W.; Benson, R., 8 - Polymeric Biomaterials A2 - Kutz, Myer. In Applied Plastics Engineering Handbook (Second Edition), William Andrew Publishing: 2017; pp 145164.

3. Mann, J. L.; Yu, A. C.; Agmon, G.; Appel, E. A., Supramolecular polymeric biomaterials. Biomater. Sci. 2018, 6 (1), 10-37.

4. Griffith, L. G., Polymeric biomaterials. Acta Materialia 2000, 48 (1), 263-277.

5. Forster, S.; Plantenberg, T., From self-organizing polymers to nanohybrid and biomaterials. Angew. Chem., Int. Ed. 2002, 41 (5), 688-714.

6. Nayak, S.; Lyon, L. A., Soft nanotechnology with soft nanoparticles. Angewandte Chemie 2005, 44 (47), 7686-708.

7. Greiner, A.; Wendorff, J. H., Elektrospinnen: eine faszinierende Methode zur Präparation ultradünner Fasern. Angewandte Chemie 2007, 119 (30), 5770-5805.

8. Groschel, A. H.; Walther, A.; Lobling, T. I.; Schacher, F. H.; Schmalz, H.; Muller, A. H., Guided hierarchical co-assembly of soft patchy nanoparticles. Nature 2013, 503 (7475), 247-51.

9. Bernstein, J.; Davis, R. E.; Shimoni, L.; Chang, N. L., Patterns in Hydrogen Bonding: Functionality and Graph Set Analysis in Crystals. Angewandte Chemie International Edition in English 1995, 34 (15), 1555-1573.

10. Lehn, J.-M., Toward complex matter: Supramolecular chemistry and selforganization. Proc. Natl. Acad. Sci. U. S. A. 2002, 99 (8), 4763-4768.

11. Lehn, J.-M., From supramolecular chemistry towards constitutional dynamic chemistry and adaptive chemistry. Chem. Soc. Rev. 2007, 36 (2), 151-160.

12. Schneider, H. J., Binding mechanisms in supramolecular complexes. Angewandte Chemie 2009, 48 (22), 3924-77.

13. Mai, Y.; Eisenberg, A., Self-assembly of block copolymers. Chem. Soc. Rev. 2012, 41 (18), 5969-5985.

14. Science

direct. https://www.sciencedirect.com/search?qs=biomaterials\&show=25\&sortBy=relev ance\&articleTypes=FLA\&lastSelectedFacet=articleTypes (accessed 11/11/2018).

15. Scifinder. https://scifinder.cas.org/scifinder/view/scifinder/scifinderExplore.jsf (accessed 03/05/2018). 
16. Kulinets, I., 1 - Biomaterials and their applications in medicine. In Regulatory Affairs for Biomaterials and Medical Devices, Amato, S. F.; Ezzell, R. M., Eds. Woodhead Publishing: 2015; pp 1-10.

17. Chuah, J.-A.; Numata, K., Stimulus-responsive peptide for effective delivery and release of DNA in plants. Biomacromolecules 2018, 19 (4), 1154-1163.

18. Nair, L. S.; Laurencin, C. T., Biodegradable polymers as biomaterials. Prog. Polym. Sci. 2007, 32 (8-9), 762-798.

19. Hoffman, A. S., Hydrogels for biomedical applications. Advanced Drug Delivery Reviews 2002, 54 (1), 3-12.

20. Mishra, M., Concise Encyclopedia of Biomedical Polymers and Polymeric Biomaterials. CRC Press: 2017; p 1867 pp.

21. Bridges, A. W.; Whitmire, R. E.; Singh, N.; Templeman, K. L.; Babensee, J. E.; Lyon, L. A.; Garcia, A. J., Chronic inflammatory responses to microgel-based implant coatings. Journal of biomedical materials research. Part A 2010, 94 (1), 252-8.

22. Peng, S.; Jin, G.; Li, L.; Li, K.; Srinivasan, M.; Ramakrishna, S.; Chen, J., Multifunctional electrospun nanofibres for advances in tissue regeneration, energy conversion \& storage, and water treatment. Chem. Soc. Rev. 2016, 45 (5), 12251241.

23. Inkinen, S.; Hakkarainen, M.; Albertsson, A.-C.; Sodergard, A., From Lactic Acid to Poly(lactic acid) (PLA): Characterization and Analysis of PLA and Its Precursors. Biomacromolecules 2011, 12 (3), 523-532.

24. Hutmacher, D. W., Scaffolds in tissue engineering bone and cartilage. Biomaterials 2000, 21 (24), 2529-2543.

25. Langer, R.; Peppas, N. A., Advances in biomaterials, drug delivery, and bionanotechnology. AIChE J. 2003, 49 (12), 2990-3006.

26. Raemdonck, K.; Demeester, J.; De Smedt, S., Advanced nanogel engineering for drug delivery. Soft Matter 2009, 5 (4), 707-715.

27. Login, R. B., N-Vinylamide Polymers. In Kirk-Othmer Encyclopedia of Chemical Technology, John Wiley \& Sons, Inc.: 2000.

28. Login, R. B. In N-vinylamide polymers, John Wiley \& Sons, Inc.: 2014; pp 315-344.

29. Kirsh, I. U. E.; Kirsh, Y. E., Water Soluble Poly-N-Vinylamides: Synthesis and Physicochemical Properties. Wiley: 1998.

30. Vihola, H.; Laukkanen, A.; Valtola, L.; Tenhu, H.; Hirvonen, J., Cytotoxicity of thermosensitive polymers poly(N-isopropylacrylamide), poly(N-vinylcaprolactam) and amphiphilically modified poly(N-vinylcaprolactam). Biomaterials 2005, 26 (16), 3055-64. 
31. Cortez-Lemus, N. A.; Licea-Claverie, A., Poly(N-vinylcaprolactam), a comprehensive review on a thermoresponsive polymer becoming popular. Progress in Polymer Science 2016, 53, 1-51.

32. Plamper, F. A.; Richtering, W., Functional Microgels and Microgel Systems. Accounts of chemical research 2017, 50 (2), 131-140.

33. Pich, A.; Richtering, W., Chemical design of responsive microgels. Springer GmbH

Berlin, Germany: 2010.

34. Pelton, R., Temperature-sensitive aqueous microgels. Adv. Colloid Interface Sci. 2000, 85 (1), 1-33.

35. Saxena, S.; Hansen, C. E.; Lyon, L. A., Microgel mechanics in biomaterial design. Accounts of chemical research 2014, 47 (8), 2426-34.

36. Schramm, L. L.; Editor, Nano- and Microtechnology From A-Z: From Nanosystems to Colloids and Interfaces. Wiley: 2014; p 392 pp.

37. Lyon, L. A.; Fernandez-Nieves, A., The polymer/colloid duality of microgel suspensions. Annu. Rev. Phys. Chem. 2012, 63, 25-43.

38. Boyko, V.; Pich, A.; Lu, Y.; Richter, S.; Arndt, K.-F.; Adler, H.-J. P., Thermo-sensitive poly(N-vinylcaprolactam-co-acetoacetoxyethyl methacrylate) microgels: 1synthesis and characterization. Polymer 2003, 44 (26), 7821-7827.

39. Berndt, I.; Pedersen, J. S.; Richtering, W., Temperature-Sensitive Core-Shell Microgel Particles with Dense Shell. Angewandte Chemie 2006, 118 (11), 17691773.

40. Lyon, L. A.; Meng, Z.; Singh, N.; Sorrell, C. D.; St John, A., Thermoresponsive microgel-based materials. Chemical Society reviews 2009, 38 (4), 865-74.

41. Schachschal, S.; Balaceanu, A.; Melian, C.; Demco, D. E.; Eckert, T.; Richtering, W.; Pich, A., Polyampholyte Microgels with Anionic Core and Cationic Shell. Macromolecules 2010, 43 (9), 4331-4339.

42. Bhattacharya, S.; Eckert, F.; Boyko, V.; Pich, A., Temperature-, pH-, and magneticfield-sensitive hybrid microgels. Small 2007, 3 (4), 650-7.

43. Phua, D. I.; Herman, K.; Balaceanu, A.; Zakrevski, J.; Pich, A., Reversible Size Modulation of Aqueous Microgels via Orthogonal or Combined Application of Thermo- and Phototriggers. Langmuir : the ACS journal of surfaces and colloids 2016, 32 (16), 3867-79.

44. Polotsky, A. A.; Plamper, F. A.; Borisov, O. V., Collapse-to-Swelling Transitions in $\mathrm{pH}^{-}$and Thermoresponsive Microgels in Aqueous Dispersions: The Thermodynamic Theory. Macromolecules 2013, 46 (21), 8702-8709.

45. Xue, B.; Kozlovskaya, V.; Kharlampieva, E., Shaped stimuli-responsive hydrogel particles: syntheses, properties and biological responses. J. Mater. Chem. B 2017, 5 (1), 9-35. 
46. Oh, J. K.; Drumright, R.; Siegwart, D. J.; Matyjaszewski, K., The development of microgels/nanogels for drug delivery applications. Prog. Polym. Sci. 2008, 33 (4), 448-477.

47. Herbert, K. M.; Schrettl, S.; Rowan, S. J.; Weder, C., 50th Anniversary Perspective: Solid-State Multistimuli, Multiresponsive Polymeric Materials. Macromolecules 2017, 50 (22), 8845-8870.

48. Wang, Y.; Zheng, J.; Tian, Y.; Yang, W., Acid degradable poly(vinylcaprolactam)based nanogels with ketal linkages for drug delivery. Journal of Materials Chemistry B 2015, 3 (28), 5824-5832.

49. Buwalda, S. J.; Vermonden, T.; Hennink, W. E., Hydrogels for Therapeutic Delivery: Current Developments and Future Directions. Biomacromolecules 2017, 18 (2), 316-330.

50. Peng, H.; Huang, X.; Oppermann, A.; Melle, A.; Weger, L.; Karperien, M.; Woell, D.; Pich, A., A facile approach for thermal and reduction dual-responsive prodrug nanogels for intracellular doxorubicin delivery. J. Mater. Chem. B 2016, 4 (47), 7572-7583.

51. Song, Q.; Gao, Y.; Xu, J.-F.; Qin, B.; Serpe, M. J.; Zhang, X., Supramolecular Microgels Fabricated from Supramonomers. ACS Macro Lett. 2016, 5 (10), 1084-1088.

52. Agrawal, G.; Wang, J.; Bruster, B.; Zhu, X.; Moller, M.; Pich, A., Degradable microgels synthesized using reactive polyvinylalkoxysiloxanes as crosslinkers. Soft Matter 2013, 9 (22), 5380-5390.

53. Schmitz, D.; Pich, A., Responsive microgels with supramolecular crosslinks: synthesis and triggered degradation in aqueous medium. Polymer Chemistry 2016, 7 (36), 5687-5697.

54. Gu, Z.; Patterson, G.; Cao, R.; Armitage, B., Self-assembled supramolecular microgels: Fractal structure and aggregation mechanism. J. Polym. Sci., Part B: Polym. Phys. 2003, 41 (23), 3037-3046.

55. Wu, Y.; Ni, P.; Zhang, M.; Zhu, X., Fabrication of microgels via supramolecular assembly of cyclodextrin-containing star polycations and oppositely charged linear polyanions. Soft Matter 2010, 6 (16), 3751-3758.

56. Rossow, T.; Bayer, S.; Albrecht, R.; Tzschucke, C. C.; Seiffert, S., Supramolecular Hydrogel Capsules Based on PEG: A Step Toward Degradable Biomaterials with Rational Design. Macromol. Rapid Commun. 2013, 34 (17), 1401-1407.

57. Webber, M. J.; Appel, E. A.; Meijer, E. W.; Langer, R., Supramolecular biomaterials. Nat. Mater. 2016, 15 (1), 13-26.

58. Whitesides, G. M.; Boncheva, M., Beyond molecules: self-assembly of mesoscopic and macroscopic components. Proceedings of the National Academy of Sciences of the United States of America 2002, 99 (8), 4769-74. 
59. Arunan, E.; Desiraju Gautam, R.; Klein Roger, A.; Sadlej, J.; Scheiner, S.; Alkorta, I.; Clary David, C.; Crabtree Robert, H.; Dannenberg Joseph, J.; Hobza, P.; Kjaergaard Henrik, G.; Legon Anthony, C.; Mennucci, B.; Nesbitt David, J., Definition of the hydrogen bond (IUPAC Recommendations 2011). In Pure and Applied Chemistry, 2011; Vol. 83, p 1637.

60. Watson, J. D.; Crick, F. H. C., Molecular Structure of Nucleic Acids: A Structure for Deoxyribose Nucleic Acid. Nature 1953, 171, 737.

61. Neidle, S., 2 - The Building-Blocks of DNA and RNA. In Principles of Nucleic Acid Structure, Academic Press: New York, 2008; pp 20-37.

62. Webber, M. J.; Langer, R., Drug delivery by supramolecular design. Chem. Soc. Rev. 2017, 46 (21), 6600-6620.

63. Correa, S.; Dreaden, E. C.; Gu, L.; Hammond, P. T., Engineering nanolayered particles for modular drug delivery. Journal of controlled release : official journal of the Controlled Release Society 2016, 240, 364-386.

64. Edgar, D. W.; Fish, J. S.; Gomez, M.; Wood, F. M., Local and systemic treatments for acute edema after burn injury: a systematic review of the literature. Journal of burn care \& research : official publication of the American Burn Association 2011, 32 (2), 334-47.

65. Campos-Vega, R.; Loarca-Pina, G.; Vergara-Castaneda, H. A.; Oomah, B. D., Spent coffee grounds: A review on current research and future prospects. Trends Food Sci. Technol. 2015, 45 (1), 24-36.

66. Quideau, S.; Deffieux, D.; Douat-Casassus, C.; Pouysegu, L., Plant polyphenols: chemical properties, biological activities, and synthesis. Angewandte Chemie 2011, 50 (3), 586-621.

67. Cheynier, V.; Yoshida, K.; Martens, S., Focus issue on polyphenols. Planta 2015, 242 (3), 507-507.

68. Quideau, S. Why bother with Polyphenols ?

http://www.groupepolyphenols.com/the-society/why-bother-with-polyphenols/.

69. Haslam, E., Natural Polyphenols (Vegetable Tannins) as Drugs: Possible Modes of Action. J. Nat. Prod. 1996, 59 (2), 205-15.

70. Nicholson, P. T.; Shaw, I.; Editors, Ancient Egyptian Materials and Technology. Cambridge Univ Press: 2000; p 702 pp.

71. Haslam, E. In Vegetable tannins, John Wiley \& Sons Ltd.: 2007; pp 984-987.

72. Chen, S.-C.; Chung, K.-T., Mutagenicity and antimutagenicity studies of tannic acid and its related compounds. Food and Chemical Toxicology 2000, 38 (1), 1-5.

73. Khan, N. S.; Ahmad, A.; Hadi, S. M., Anti-oxidant, pro-oxidant properties of tannic acid and its binding to DNA. Chemico-Biological Interactions 2000, 125 (3), 177189. 
74. Erel-Unal, I.; Sukhishvili, S. A., Hydrogen-Bonded Multilayers of a Neutral Polymer and a Polyphenol. Macromolecules 2008, 41 (11), 3962-3970.

75. Sulaiman, M.; Tijani, H. I.; Abubakar, B. M.; Haruna, S.; Hindatu, Y.; Mohammed, J. N.; Idris, A., An overview of natural plant antioxidants: analysis and evaluation. $A d v$. Biochem. 2013, 1 (4), 64-72.

76. Giglio, R. V.; Patti, A. M.; Cicero, A. F. G.; Lippi, G.; Rizzo, M.; Toth, P. P.; Banach, M., Polyphenols: Potential Use in the Prevention and Treatment of Cardiovascular Diseases. Curr. Pharm. Des. 2018, 24 (2), 239-258.

77. Menaa, F.; Menaa, A.; Tréton, J., Chapter 63 - Polyphenols against Skin Aging. In Polyphenols in Human Health and Disease, Academic Press: San Diego, 2014; pp 819-830.

78. Giampieri, F.; Forbes-Hernandez, T. Y.; Gasparrini, M.; Alvarez-Suarez, J. M.; Afrin, S.; Bompadre, S.; Quiles, J. L.; Mezzetti, B.; Battino, M., Strawberry as a health promoter: an evidence based review. Food Funct. 2015, 6 (5), 1386-1398.

79. Shahidi, F.; Ambigaipalan, P., Phenolics and polyphenolics in foods, beverages and spices: Antioxidant activity and health effects: A review. J. Funct. Foods 2015, 18 (Part_B), 820-897.

80. Caruso, F.; Ejima, H.; Richardson, J. J.; Van Koeverden, M. P. One step assembly of metal-polyphenol complexes for versatile film and particle capsules engineering. W02014197940A1, 2014.

81. Rahim, M. A.; Bjoernmalm, M.; Suma, T.; Faria, M.; Ju, Y.; Kempe, K.; Muellner, M.; Ejima, H.; Stickland, A. D.; Caruso, F., Metal-Phenolic Supramolecular Gelation. Angew. Chem., Int. Ed. 2016, 55 (44), 13803-13807.

82. Fan, H.; Wang, J.; Zhang, Q.; Jin, Z., Tannic Acid-Based Multifunctional Hydrogels with Facile Adjustable Adhesion and Cohesion Contributed by Polyphenol Supramolecular Chemistry. ACS Omega 2017, 2 (10), 6668-6676.

83. Fan, H.; Wang, L.; Feng, X.; Bu, Y.; Wu, D.; Jin, Z., Supramolecular Hydrogel Formation Based on Tannic Acid. Macromolecules 2017, 50 (2), 666-676.

84. Lomova, M. V.; Brichkina, A. I.; Kiryukhin, M. V.; Vasina, E. N.; Pavlov, A. M.; Gorin, D. A.; Sukhorukov, G. B.; Antipina, M. N., Multilayer Capsules of Bovine Serum Albumin and Tannic Acid for Controlled Release by Enzymatic Degradation. ACS applied materials \& interfaces 2015, 7 (22), 11732-40.

85. Ninan, N.; Forget, A.; Shastri, V. P.; Voelcker, N. H.; Blencowe, A., Anti-bacterial and anti-inflammatory pH-responsive tannic acid - carboxylated agarose composite hydrogels for wound healing. ACS applied materials \& interfaces 2016, 8 (42), 28511-28521.

86. Shukla, A.; Fang, J. C.; Puranam, S.; Jensen, F. R.; Hammond, P. T., Hemostatic multilayer coatings. Advanced materials 2012, 24 (4), 492-6. 
87. Shutava, T. G.; Balkundi, S. S.; Vangala, P.; Steffan, J. J.; Bigelow, R. L.; Cardelli, J. A.; O'Neal, D. P.; Lvov, Y. M., Layer-by-Layer-Coated Gelatin Nanoparticles as a Vehicle for Delivery of Natural Polyphenols. ACS Nano 2009, 3 (7), 1877-1885.

88. Sieniawska, E.; Baj, T., Chapter 10 - Tannins A2 - Badal, Simone. In Pharmacognosy, Delgoda, R., Ed. Academic Press: Boston, 2017; pp 199-232.

89. Beck, C. S.; Powers, J. H., BURNS TREATED BY TANNIC ACID. Ann Surg 1926, 84 (1), 19-36.

90. Raquel, P.; Laura, B.; Fulgencio, S.-C., Antioxidant Activity of Dietary Polyphenols as Determined by a Modified Ferric Reducing Antioxidant Power Assay. Journal of Agricultural and Food Chemistry 2000, 48 (8), 3396-3402.

91. Caruso, F.; Ejima, H.; Richardson, J. J.; Van Koeverden, M. P. One step assembly of metal-polyphenol complexes for versatile film and particle capsules engineering. WO2014197940A1, 2014.

92. Maerten, C.; Lopez, L.; Lupattelli, P.; Rydzek, G.; Pronkin, S.; Schaaf, P.; Jierry, L.; Boulmedais, F., Electrotriggered Confined Self-assembly of Metal-Polyphenol Nanocoatings Using a Morphogenic Approach. Chem. Mater. 2017, Ahead of Print.

93. Chung, K.-T.; Wong, T. Y.; Wei, C.-I.; Huang, Y.-W.; Lin, Y., Tannins and human health: a review. Crit. Rev. Food Sci. Nutr. 1998, 38 (6), 421-464.

94. Liu, R.; Zheng, J.; Guo, R.; Luo, J.; Yuan, Y.; Liu, X., Synthesis of New Biobased Antibacterial Methacrylates Derived from Tannic Acid and Their Application in UVCured Coatings. Industrial \& Engineering Chemistry Research 2014, 53 (27), 1083510840.

95. Sanchez-Moreno, C.; Larrauri, J. A.; Saura-Calixto, F., A procedure to measure the antiradical efficiency of polyphenols. J. Sci. Food Agric. 1998, 76 (2), 270-276.

96. Shutava, T.; Prouty, M.; Kommireddy, D.; Lvov, Y., pH responsive decomposable layer-by-layer nanofilms and capsules on the basis of tannic acid. Macromolecules 2005, 38 (7), 2850-2858.

97. Kozlovskaya, V.; Harbaugh, S.; Drachuk, I.; Shchepelina, O.; Kelley-Loughnane, N.; Stone, M.; Tsukruk, V. V., Hydrogen-bonded LbL shells for living cell surface engineering. Soft Matter 2011, 7 (6), 2364-2372.

98. Conte, M. P.; Lau, K. H. A.; Ulijn, R. V., Biocatalytic Self-Assembly Using Reversible and Irreversible Enzyme Immobilization. ACS Appl. Mater. Interfaces 2017, 9 (4), 3266-3271.

99. Braidy, N.; Jugder, B.-E.; Poljak, A.; Jayasena, T.; Nabavi, S. M.; Sachdev, P.; Grant, R., Molecular Targets of Tannic Acid in Alzheimer';s Disease. Curr. Alzheimer Res. 2017, 14 (8), 861-869.

100. Han, L.; Liu, Q.; Yang, L.; Ye, T.; He, Z.; Jia, L., Facile Oriented Immobilization of Histidine-Tagged Proteins on Nonfouling Cobalt Polyphenolic Self-Assembly Surfaces. ACS Biomater. Sci. Eng. 2017, 3 (12), 3328-3337. 
101. Sahiner, N.; Sagbas, S.; Sahiner, M.; Silan, C., P(TA) macro-, micro-, nanoparticleembedded super porous p(HEMA) cryogels as wound dressing material. Mater. Sci. Eng., C 2017, 70 (Part_1), 317-326.

102. Liu, F.; Kozlovskaya, V.; Zavgorodnya, O.; Martinez-Lopez, C.; Catledge, S.; Kharlampieva, E., Encapsulation of anticancer drug by hydrogen-bonded multilayers of tannic acid. Soft Matter 2014, 10 (46), 9237-9247.

103. Park, J. H.; Hong, D.; Lee, J.; Choi, I. S., Cell-in-Shell Hybrids: Chemical Nanoencapsulation of Individual Cells. Acc. Chem. Res. 2016, 49 (5), 792-800.

104. Ping, Y.; Guo, J.; Ejima, H.; Chen, X.; Richardson, J. J.; Sun, H.; Caruso, F., pHResponsive capsules engineered from metal-phenolic networks for anticancer drug delivery. Small 2015, 11 (17), 2032-2036.

105. Chapter 1 Introduction. In Non-Covalent Interactions: Theory and Experiment, The Royal Society of Chemistry: 2009; pp 1-20.

106. Chapter 4 Classification of Non-covalent Complexes. In Non-Covalent Interactions: Theory and Experiment, The Royal Society of Chemistry: 2009; pp 134-154.

107. Shin, M.; Ryu, J. H.; Park, J. P.; Kim, K.; Yang, J. W.; Lee, H., DNA/tannic acid hybrid gel exhibiting biodegradability, extensibility, tissue adhesiveness, and hemostatic Ability. Adv. Funct. Mater. 2015, 25 (8), 1270-1278.

108. Jeffrey, G. A. J.; Jeffrey, G. A., An Introduction to Hydrogen Bonding. Oxford University Press: 1997.

109. Imaz, A.; Miranda, J. I.; Ramos, J.; Forcada, J., Evidences of a hydrolysis process in the synthesis of N-vinylcaprolactam-based microgels. Eur. Polym. J. 2008, 44 (12), 4002-4011.

110. Gülçin, İ.; Huyut, Z.; Elmastaş, M.; Aboul-Enein, H. Y., Radical scavenging and antioxidant activity of tannic acid. Arabian Journal of Chemistry 2010, 3 (1), 43-53.

111. Ejima, H.; Richardson, J. J.; Caruso, F., Phenolic film engineering for templatemediated microcapsule preparation. Polym J 2014, 46 (8), 452-459.

112. Kim, B. S.; Lee, H. I.; Min, Y.; Poon, Z.; Hammond, P. T., Hydrogen-bonded multilayer of $\mathrm{pH}$-responsive polymeric micelles with tannic acid for surface drug delivery. Chemical communications 2009, (28), 4194-6.

113. Xue, J.; Zhang, Z.; Nie, J.; Du, B., Formation of Microgels by Utilizing the Reactivity of Catechols with Radicals. Macromolecules (Washington, DC, U. S.) 2017, 50 (14), 5285-5292.

114. Kozlovskaya, V.; Kharlampieva, E.; Drachuk, I.; Cheng, D.; Tsukruk, V. V., Responsive microcapsule reactors based on hydrogen-bonded tannic acid layer-by-layer assemblies. Soft Matter 2010, 6 (15), 3596.

115. Molano Lopez, C.; Pich, A., Supramolecular Stimuli-Responsive Microgels Crosslinked by Tannic Acid. Macromol Rapid Commun 2018. 
116. Melle, A.; Balaceanu, A.; Kather, M.; Wu, Y.; Gau, E.; Sun, W.; Huang, X.; Shi, X.; Karperien, M.; Pich, A., Stimuli-responsive poly(N-vinylcaprolactam-co-2methoxyethyl acrylate) core-shell microgels: facile synthesis, modulation of surface properties and controlled internalisation into cells. J. Mater. Chem. B 2016, 4 (30), 5127-5137.

117. Pantoja-Castro, M. A.; González-Rodríguez, H., Study by infrared spectroscopy and thermogravimetric analysis of Tannins and Tannic acid. Revista latinoamericana de química 2011, 39, 107-112.

118. Kehren, D.; Molano Lopez, A. C.; Pich, A., Nanogel-modified polycaprolactone microfibres with controlled water uptake and degradability. Polymer 2014, 55 (9), 2153-2162.

119. Tieke, B., Makromolekulare Chemie: Eine Einführung. Wiley: 1997.

120. https://www.encyclopedia.com/people/science-and-technology/chemistrybiographies/soren-peter-lauritz-sorensen (accessed 04/06/2018).

121. Bates, R. G., Definitions of pH Scales. Chemical reviews 1948, 42 (1), 1-61.

122. Bates, R. G., Determination of pH: theory and practice. Wiley: 1973.

123. Covington, A. K.; Bates, R. G.; Durst, R. A., Definition of pH scales, standard reference values, measurement of $\mathrm{pH}$ and related terminology (Recommendations 1984). In Pure and Applied Chemistry, 1985; Vol. 57, p 531.

124. Myers, R. J., One-Hundred Years of pH. Journal of Chemical Education 2010, 87 (1), 30-32.

125. Lim, K. F., Negative pH Does Exist. Journal of Chemical Education 2006, 83 (10), 1465.

126. Casey, J. R.; Grinstein, S.; Orlowski, J., Sensors and regulators of intracellular pH. Nature Reviews Molecular Cell Biology 2009, 11, 50.

127. Han, J.; Burgess, K., Fluorescent Indicators for Intracellular pH. Chemical reviews 2010, 110 (5), 2709-2728.

128. Liu, J.; Huang, Y.; Kumar, A.; Tan, A.; Jin, S.; Mozhi, A.; Liang, X.-J., pH-Sensitive nanosystems for drug delivery in cancer therapy. Biotechnology advances 2014, 32 (4), 693-710.

129. Kanamala, M.; Wilson, W. R.; Yang, M.; Palmer, B. D.; Wu, Z., Mechanisms and biomaterials in pH-responsive tumour targeted drug delivery: A review. Biomaterials 2016, 85, 152-167.

130. Pang, X.; Jiang, Y.; Xiao, Q.; Leung, A. W.; Hua, H.; Xu, C., pH-responsive polymerdrug conjugates: Design and progress. Journal of Controlled Release 2016, 222, 116-129.

131. Li, Y.; Wang, Y.; Huang, G.; Gao, J., Cooperativity Principles in Self-Assembled Nanomedicine. Chemical reviews 2018. 
132. Modi, S.; M. G, S.; Goswami, D.; Gupta, G. D.; Mayor, S.; Krishnan, Y., A DNA nanomachine that maps spatial and temporal pH changes inside living cells. Nature Nanotechnology 2009, 4, 325.

133. Peng, W., Nature\&\#39;s pH meter. Nature Methods 2009, 6, 404.

134. Fehér, K.; Romstadt, T.; Böhm, C. A.; Kolkenbrock, M.; Blau, M. F.; Kuehlwetter, J.; Molano Lopez, A. C.; Pich, A.; Hannen, J.; Bürgermeister, L.; Schaaps, N.; Vogt, F.; Gries, T.; Jockenhövel, S., Microgel-functionalised fibres with pH-optimised degradation behaviour - a promising approach for short-term medical applications. BioNanoMaterials 2015, 16 (4).

135. Barbasz, A.; Ocwieja, M.; Barbasz, J., Cytotoxic Activity of Highly Purified Silver Nanoparticles Sol Against Cells of Human Immune System. 2015; Vol. 176.

136. Panyachariwat, N.; Steckel, H., Stability of urea in solution and pharmaceutical preparations. Journal of cosmetic science 2014, 65 (3), 187-95.

137. Raab, R. P., Urea from the chemist's point of view. Journal of Applied Cosmetology 1991, 9, 9-13.

138. Word Health Organization. http://www.who.int (accessed 21/06/2018).

139. Hammond, P. T., Nano Tools Pave the Way to New Solutions in Infectious Disease. ACS Infect. Dis. 2017, 3 (8), 554-558.

140. Senapati, S.; Mahanta, A. K.; Kumar, S.; Maiti, P., Controlled drug delivery vehicles for cancer treatment and their performance. Signal Transduction and Targeted Therapy 2018, 3 (1), 7.

141. Haag, R., Supramolecular drug-delivery systems based on polymeric core-shell architectures. Angew. Chem., Int. Ed. 2004, 43 (3), 278-282.

142. Peer, D.; Karp, J. M.; Hong, S.; Farokhzad, O. C.; Margalit, R.; Langer, R., Nanocarriers as an emerging platform for cancer therapy. Nature Nanotechnology 2007, 2, 751.

143. Luo, L.; Tam, J.; Maysinger, D.; Eisenberg, A., Cellular Internalization of Poly(ethylene oxide)-b-poly( $\varepsilon$-caprolactone) Diblock Copolymer Micelles. Bioconjugate chemistry 2002, 13 (6), 1259-1265.

144. Nowag, S.; Haag, R., pH-Responsive Micro- and Nanocarrier Systems. Angew. Chem., Int. Ed. 2014, 53 (1), 49-51.

145. Fleige, E.; Quadir, M. A.; Haag, R., Stimuli-responsive polymeric nanocarriers for the controlled transport of active compounds: Concepts and applications. Adv. Drug Delivery Rev. 2012, 64 (9), 866-884.

146. Zhang, Y.; Ang, C. Y.; Zhao, Y., Polymeric nanocarriers incorporating near-infrared absorbing agents for potent photothermal therapy of cancer. Polym $J$ 2015, 48, 589.

147. Younsoo, B.; Shigeto, F.; Atsushi, H.; Kazunori, K., Design of Environment-Sensitive Supramolecular Assemblies for Intracellular Drug Delivery: Polymeric Micelles 
that are Responsive to Intracellular pH Change. Angewandte Chemie International Edition 2003, 42 (38), 4640-4643.

148. Alipour, M.; Hosseinkhani, S.; Sheikhnejad, R.; Cheraghi, R., Nano-biomimetic carriers are implicated in mechanistic evaluation of intracellular gene delivery. Scientific Reports 2017, 7, 41507.

149. Dimde, M.; Neumann, F.; Reisbeck, F.; Ehrmann, S.; Cuellar-Camacho, J. L.; Steinhilber, D.; Ma, N.; Haag, R., Defined pH-sensitive nanogels as gene delivery platform for siRNA mediated in vitro gene silencing. Biomater. Sci. 2017, 5 (11), 2328-2336.

150. Steinhilber, D.; Rossow, T.; Wedepohl, S.; Paulus, F.; Seiffert, S.; Haag, R., A Microgel Construction Kit for Bioorthogonal Encapsulation and pH-Controlled Release of Living Cells. Angew. Chem., Int. Ed. 2013, 52 (51), 13538-13543.

151. Raghupathi, K. R.; Koodali, R. T.; Manna, A. C., Size-dependent bacterial growth inhibition and mechanism of antibacterial activity of zinc oxide nanoparticles. Langmuir : the ACS journal of surfaces and colloids 2011, 27 (7), 4020-8.

152. Blanco-Andujar, C.; Tung, L. D.; Thanh, N. T. K., Synthesis of nanoparticles for biomedical applications. Annu. Rep. Prog. Chem., Sect. A: Inorg. Chem. 2010, 106, 553-568.

153. Bernát, I., The Distribution of Iron in Nature. In Iron Metabolism, Springer US: Boston, MA, 1983; pp 9-13.

154. Connorton, J. M.; Balk, J.; Rodriguez-Celma, J., Iron homeostasis in plants - a brief overview. Metallomics 2017, 9 (7), 813-823.

155. Puig, S.; Ramos-Alonso, L.; Romero, A. M.; Martinez-Pastor, M. T., The elemental role of iron in DNA synthesis and repair. Metallomics 2017, 9 (11), 1483-1500.

156. Pittman, R. N., Regulation of Tissue Oxygenation, Second Edition. Biota Publishing: 2016.

157. Roth-Walter, F.; Pacios, L. F.; Bianchini, R.; Jensen-Jarolim, E., Linking irondeficiency with allergy: role of molecular allergens and the microbiome. Metallomics 2017, 9 (12), 1676-1692.

158. Li, H.; Zhao, H.; Hao, S.; Shang, L.; Wu, J.; Song, C.; Meyron-Holtz, E. G.; Qiao, T.; Li, K., Iron regulatory protein deficiency compromises mitochondrial function in murine embryonic fibroblasts. Scientific Reports 2018, 8 (1), 5118.

159. Meurer, R. A.; Kemper, S.; Knopp, S.; Eichert, T.; Jakob, F.; Goldbach, H. E.; Schwaneberg, U.; Pich, A., Biofunctional Microgel-Based Fertilizers for Controlled Foliar Delivery of Nutrients to Plants. Angew. Chem., Int. Ed. 2017, 56 (26), 73807386.

160. Yilmaz, Y.; Toledo, R. T., Major Flavonoids in Grape Seeds and Skins: Antioxidant Capacity of Catechin, Epicatechin, and Gallic Acid. J. Agric. Food Chem. 2004, 52 (2), 255-260. 
161. Nune, S. K.; Chanda, N.; Shukla, R.; Katti, K.; Kulkarni, R. R.; Thilakavathy, S.; Mekapothula, S.; Kannan, R.; Katti, K. V., Green nanotechnology from tea: phytochemicals in tea as building blocks for production of biocompatible gold nanoparticles. J. Mater. Chem. 2009, 19 (19), 2912-2920.

162. Sedo, J.; Saiz-Poseu, J.; Busque, F.; Ruiz-Molina, D., Catechol-Based Biomimetic Functional Materials. Adv. Mater. (Weinheim, Ger.) 2013, 25 (5), 653-701.

163. Li, H.; Chen, Q.; Zhao, J.; Urmila, K., Enhancing the antimicrobial activity of natural extraction using the synthetic ultrasmall metal nanoparticles. Scientific Reports 2015, 5, 11033.

164. Yang, Y.; Qin, Y. J.; Yip, Y. W. Y.; Chan, K. P.; Chu, K. O.; Chu, W. K.; Ng, T. K.; Pang, C. P.; Chan, S. O., Green tea catechins are potent anti-oxidants that ameliorate sodium iodate-induced retinal degeneration in rats. Scientific Reports 2016, 6, 29546.

165. Matsuzaki, T.; Ito, H.; Chevyreva, V.; Makky, A.; Kaufmann, S.; Okano, K.; Kobayashi, N.; Suganuma, M.; Nakabayashi, S.; Yoshikawa, H. Y.; Tanaka, M., Adsorption of galloyl catechin aggregates significantly modulates membrane mechanics in the absence of biochemical cues. Physical Chemistry Chemical Physics 2017, 19 (30), 19937-19947.

166. Yee, E. M. H.; Brandl, M. B.; Pasquier, E.; Cirillo, G.; Kimpton, K.; Kavallaris, M.; Kumar, N.; Vittorio, O., Dextran-Catechin inhibits angiogenesis by disrupting copper homeostasis in endothelial cells. Scientific Reports 2017, 7 (1), 7638.

167. Krol, E.; de, S. B. A.; Scheffers, D.-J.; da, S. I.; Ferreira, H.; Polaquini, C. R.; Regasini, L. O., Antibacterial activity of alkyl gallates is a combination of direct targeting of FtsZ and permeabilization of bacterial membranes. Front Microbiol 2015, 6, 390.

168. Silva, I. C.; Polaquini, C. R.; Regasini, L. O.; Ferreira, H.; Pavan, F. R., Evaluation of cytotoxic, apoptotic, mutagenic, and chemopreventive activities of semi-synthetic esters of gallic acid. Food Chem. Toxicol. 2017, 105, 300-307.

169. Silva, I. C.; Regasini, L. O.; Petronio, M. S.; Silva, D. H. S.; Bolzani, V. S.; Belasque, J., Jr.; Sacramento, L. V. S.; Ferreira, H., Antibacterial activity of alkyl gallates against Xanthomonas citri subsp. citri. J. Bacteriol. 2013, 195 (1), 85-94.

170. Gottwald, T. R.; Graham, J. H.; Schubert, T. S., Citrus Canker: The Pathogen and Its Impact. Plant health progress 2002, 2002, pp. 54-59.

171. Brunings, A. M.; Gabriel, D. W., Xanthomonas citri: breaking the surface. Molecular plant pathology 2003, 4 (3), 141-157.

172. Saiz-Poseu, J.; Mancebo-Aracil, J.; Nador, F.; Busque, F.; Ruiz-Molina, D., The Chemistry behind Catechol-Based Adhesion. Angew. Chem., Int. Ed. 2018, Ahead of Print.

173. Jakob, F.; Pich, A.; Ruebsam, K.; Conrath, U.; Soezer, N.; Schwaneberg, U. Plant protection and/or plant growth promotional system. W02016134806A1, 2016. 


\section{LIST OF PUBLICATIONS AND CONFERENCE CONTRIBUTIONS}

\section{Scientific Publications}

Parts of this doctoral work have been published in a modified form in the following article:

- Molano López, C., Pich, A., (2018) “Supramolecular Stimuli-Responsive Microgels CrossLinked by Tannic Acid", Macromolecular Rapid Communications, 39, 1700808.

\section{Conference Contributions}

Results of this doctoral research have been presented in form of an oral presentation or poster in the following conferences:

- 2nd XanthoMeeting 2018 (São Paulo, Brazil-2018): "Supramolecular StimuliResponsive Microgels Based on a Bioactive Natural Polyphenol" (poster) and “Polymeric Materials as Responsive Nanocarriers" (oral presentation).

- CARIBMAT 2018 - 2nd Caribbean Conference on Functional Materials (Cartagena, Colombia-2018): "Supramolecular and Responsive Microgels" (oral presentation).

- 7th International Colloids Conference (Barcelona, Spain-2017): “T-Responsive and pHDegradable H-Bonded Microgels Based on a Natural Polyphenol"' (poster). 